ANL-5567 Rev.

Physics

AEC Research and

Development Report

ARGONNE NATIONAL LABORATORY

9700 South Cass Avenue

Argonne, Illinois

NEUTRON SCATTERING ANGULAR DISTRIBUTION

by

A. Langsdorf, Jr., R. O. Lane, and J. E. Monahan

Physics Division

First Published: June 1956

Revised: October 1961

Operated by The University of Chicago

under

Contract W-31-109-eng-38 


\section{DISCLAIMER}

This report was prepared as an account of work sponsored by an agency of the United States Government. Neither the United States Government nor any agency Thereof, nor any of their employees, makes any warranty, express or implied, or assumes any legal liability or responsibility for the accuracy, completeness, or usefulness of any information, apparatus, product, or process disclosed, or represents that its use would not infringe privately owned rights. Reference herein to any specific commercial product, process, or service by trade name, trademark, manufacturer, or otherwise does not necessarily constitute or imply its endorsement, recommendation, or favoring by the United States Government or any agency thereof. The views and opinions of authors expressed herein do not necessarily state or reflect those of the United States Government or any agency thereof. 


\section{DISCLAIMER}

Portions of this document may be illegible in electronic image products. Images are produced from the best available original document. 


\section{PREFACE}

This report was originally issued in 1956 as Argonne National Laboratory Report ANL-5567. A compressed form of the data was subsequently published in the Physical Review. [Langsdorf, Lane, and Monahan, Phys. Rev. 107, 1077-1087(1957).] It is easier to use the larger curves in this ANL report as a source of actual numerical data; and, in addition, experimental points are given as well as curves. For these reasons and because it is unlikely that an equally comprehensive survey will soon supplant these data, the report is being reprinted.

Proper caution in interpretation of the data in the graphs on pages 57-137 requires careful reading of the text, especially the abstract (pp. 3 and 4) and Sec. VI (pp. 53 and 54). Note especially the estimated accuracy of the values of $\sigma_{t}$ and $\omega_{l}$ (p. 3), the large uncertainty in the correct energy for the points located at 30 and $60 \mathrm{kev}$ (p. 54), and the manner in which the curves were drawn (p. 4). Note the increased uncertainty for "canned" samples as mentioned on p. 60 and elsewhere. Also note that after the curves were drawn, some points were recalculated with geometric mean scattering angles replaced by weighted mean scattering angles, as discussed in the third paragraph on p. 50. Many graphs show such recorrected points which usually indicate that the curves were drawn at a bit too low a value of the argument $\sigma_{t}$ or $\omega_{l}$.

Some typographical errors in the original report have been corrected in this reprinting.

Apparatus of the type used in this work has now been described more fully by one of us [A. Langsdorf, Jr., "Neutron Collimation and Shielding for Experimental Purposes," Chap. IV.E of Fast Neutron Physics, Part I, edited by J. B. Marion and J. L. Fowler (Interscience Publishers, Inc., New York, 1960), pp. 721-806].

We have published additional experimental data [R. O. Lane, A. Langsdorf, Jr., J. D. Monahan, and A. J. Elwyn, Ann. of Phys. 12, 135-171 (1961)] which are summarized in an appendix to this report. A comparison of the newer data with the older data, where there is overlap, usually indicates good agreement. The measurement of counter efficiency, discussed on p. 51 of this report, has now been carried out as described in the Annals article, pp. 141-144. The results show that the statement in the second paragraph of page 3 in this report is erroneous; the efficiency is not constant within $10 \%$. It is therefore preferable to use the newer data for carbon and beryllium as given in the Annals article because it has been corrected for the variation of counter efficiency with energy. It might be desirable to apply a similar correction to the data in this report for boron and a few other light elements. 
Some of the curves for $\omega_{1}$ vs energy were drawn in such a way that there appeared to be a parabolic curvature near zero energy. The plots of $B_{1}$ vs energy do not bear out the existence of any such curvature.

All the data in this report are for neutrons of quite broad energy spread (p. 3). Some of the newer data are for a much narrower spread. Unfortunately, it is impractical to make an extensive survey with high resolution so that this low-resolution data will be all the information available for many materials for some time to come. 
TABLE OF CONTENTS

Page

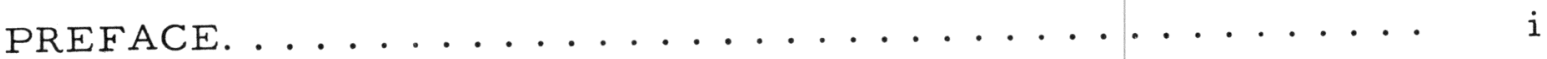

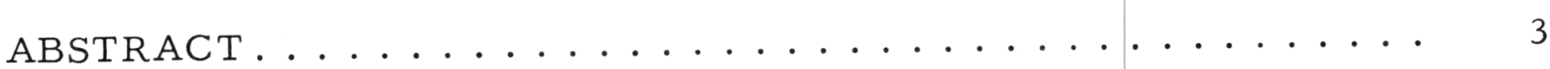

SECTION I: EXPERIMENTAL APPARATUS. ........... 5

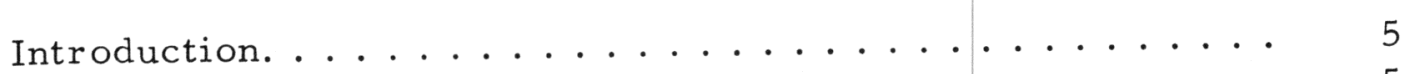

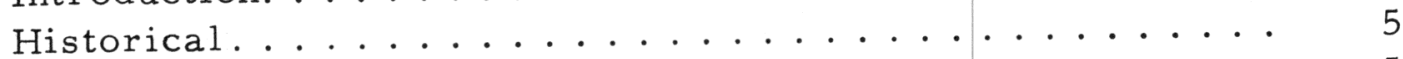

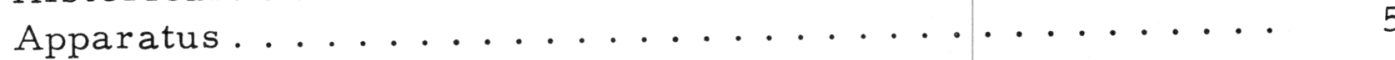

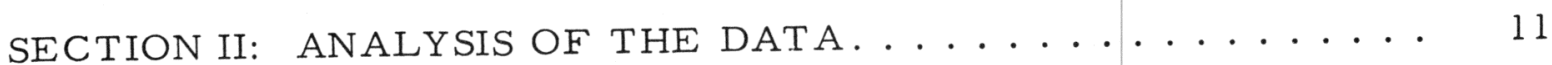

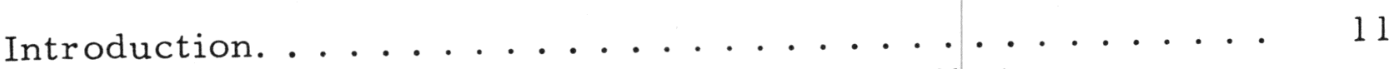

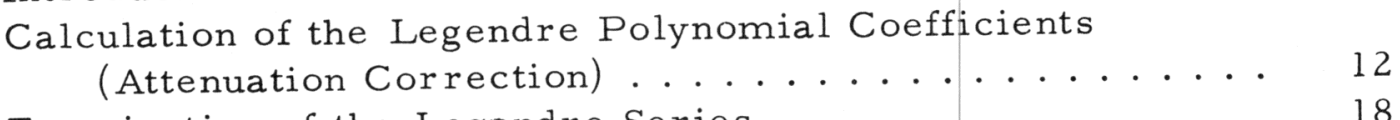

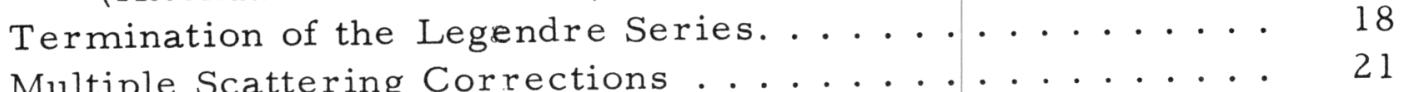

SECTION III: SINGLE AND DOUBLE SCATTERING IN PLATES. . . 36

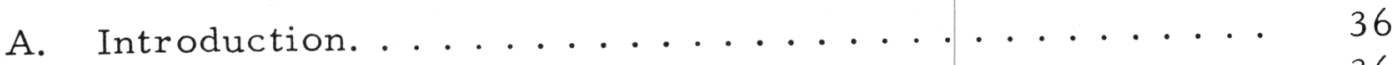

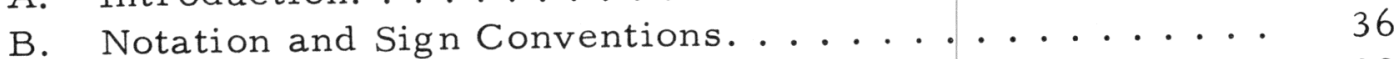

C. Idealized Singly Scattered Intensity . . . . . . . . . . . 38

D. Emergent Singly Scattered Intensity. . . . . . . . . . 39

E. Integrated Emergent Single Scattering . . . . . . . . . 40

F. Emergent Doubly Scattered Intensity . . . . . . . . . . 42

G. The Integrated Doubly Scattered Flux. . . . . . . 48

SECTION IV: PLANS FOR FUTURE ANGULAR DISTRIBUTION

DATA (The Angular Resolution Correction)..... 50

SECTION V: ACKNOWLEDGEMENTS . . . . . . . . . . . . . 52

SECTION VI: THE EXPERIMENTAL DATA............ 53

APPENDIX A. A GENERALIZED EXPONENTIAL INTEGRAL . . . 146

APPENDIX B. THE FUNCTIONS $F_{j}(\tau, \alpha), F_{j}(\tau, \alpha, s)$ and $M_{0}(\tau, \alpha) . .155$

APPENDIX C. THE INTEGRAL I I $I_{S, j+1}^{(1)}(h, \alpha) \ldots \ldots 158$

APPENDIX D. THE INTEGRAL I I $\mathrm{I}_{\mathrm{j}, \mathrm{j}+1}(\mathrm{~h}, \alpha) \ldots \ldots \ldots 16 \ldots$

APPENDIX E. SUMMARY OF THE NEWER DATA PUBLISHED IN THE ANNALS OF PHYSICS........... 164 


\title{
NEUTRON SCATTERING ANGULAR DISTRIBUTION
}

\author{
A. Langsdorf, Jr., R. O. Lane, J. Monahan
}

\section{ABSTRACT}

The accompanying 41 graphs on pp. 57-137 present the results of a survey of the angular distribution of neutrons scattered from 36 elements, two compounds, and one alloy. The data are presented in the form of an expansion in a series of Legendre polynomials,

$$
\sigma(\theta)=\frac{\sigma_{t}}{4 \pi} \sum_{l}^{\stackrel{4}{=}} \omega_{l} \quad P_{l}(\cos \theta),
$$

in the laboratory coordinate system. The graphs give $\sigma_{t}, \omega_{1}, \omega_{2}, \omega_{3}$, and $\omega_{4}$ as a function of neutron energy.

Since the detector system used $\mathrm{BF}_{3}$ proportional counters immersed in an oil moderator so arranged as to have constant (within 10\%) counting efficiency for neutrons of all energies from $100 \mathrm{kev}$ to $1500 \mathrm{kev}$, $\sigma(\theta)$ is the total differential scattering cross section.

The actual scattering detectors were positioned at five scattering angles: $22^{\circ}, 54^{\circ}, 92^{\circ}$ (on the transmission side of the plate scatterer), $112^{\circ}$ and $144^{\circ}$ (on the reflection side). Therefore $\sigma(\theta)$, as given by the above expansion, will be correct at these angles even if the value thus extrapolated to other angles is slightly erroneous.

The angular distribution is measured usually at about $80-\mathrm{kev}$ intervals for each element; the resolution was $100 \mathrm{kev}$ at the lower energies, decreasing to $\sim 60 \mathrm{kev}$ at the higher incident energies. For the transmission angles the angular resolution is $\sim 5^{\circ}$, while for the reflection angles it is $\sim 3^{\circ}$.

A statistical analysis of part of the data, as well as an observation of the general consistency, indicated standard deviations of $\sim 0.05$ for $\omega_{1}$ and \pm 0.10 for $\omega_{4}$, with the accuracy of $\omega_{2}$ and $\omega_{3}$ being intermediate be tween these values. These deviations are roughly independent of the values of the $\omega_{l}$. In most cases the total cross section, $\sigma_{t}$, is probably accurate to better than $10 \%$ statistically.

It was necessary to "can" some eight materials in containers of 0.005 -in. shim stock steel. For these samples the background counting rates - measured with an empty can in place of the scatterer - were 
higher than in other cases, leading to slightly less accurate results statistically and also to slightly larger systematic errors.

All total cross sections are evaluated in terms of the total cross section of a given sample of carbon, the value of which was taken from BNL-325 and unpublished data.

On the graphs open circles are used for $\sigma_{t}$ and $\omega_{4}$, open triangles $(\nabla)$ for $\omega_{1}$, open squares for $\omega_{2}$, and open triangles $(\Delta)$ for $\omega_{3}$. The re is no graph for $\mathbb{N}_{0}$, which was assumed to be equal to unity; this is equivalent to neglecting all reactions other than neutron scattering.

The data points with open symbols are not corrected for plural scattering. The corresponding solid symbols represent points corrected for plural scattering as described in this report. Such corrections were made on part of the data for twelve of the elements. For the se twelve samples the solid lines were drawn following the shape of the uncorrected data but displaced by an amount indicated by the corrected points. Thus the solid lines are the best indication of the true behavior of $\omega_{l}$ over the entire energy region covered. On the other graphs the solid lines were drawn by "guestimation" - a combination of quantitative estimation plus some guessing - by analogy with the twelve cases for which corrections had been made. The solid lines on these graphs are a reasonable approximation to the correct $\sigma_{t}$ and $\omega l$, but are less accurate than in the cases where the actual corrections were made. No line is drawn for $\omega_{3}$ and/or $\omega_{4}$ when either is zero within the accuracy of the data.

In several graphs data is given for two or more thicknesses of scattering sample at the same neutron energy. In some of the se cases $\left({ }_{6} \mathrm{C},{ }_{11} \mathrm{Na},{ }_{12} \mathrm{Mg},{ }_{47} \mathrm{Ag}\right.$, and $\left.{ }_{92} \mathrm{U}\right)$ several thicknesses were corrected for plural scattering.

The remainder of this report describes the experiment and the analytical methods which were used to obtain corrected cross sections in the representation shown in the graphs. Section I contains a detailed description of the apparatus and of the experimental techniques which were used. In Section II the analytical methods of obtaining a microscopic differential cross section from the raw data are described. Section III contains a discussion of the plural scattering corrections for this geometry (a collimated beam of particles incident on a thin flat scatterer). Section IV discusses some modifications, both in experimental procedure and analytical treatment of the data, which are planned for future experiments of this type. Section V contains a list of those people who have contributed to the various phases of this work. Finally, in Section VI the symbolism of the graphical presentation of the differential cross section data is explained. 


\section{SECTION I.}

\section{EXPERIMENTAL APPARATUS}

\section{General Purpose; Introduction}

The determination of the angular distribution of scattered monoenergetic neutrons is of interest and importance from various points of view. The lack of agreement between experiments and relevant theories may well be resolved in the light of more extensive experimental information. These discrepancies, particularly in theories which average over a band of energies including many resonances, are of current interest. In addition, angular distribution information in the neighborhood of individual resolvable resonances (in the lighter elements, particularly) is a powerful aid in the assignment of correct angular momenta associated with such resonances. Finally, such data have a wide range of practical uses in the solution of transport problems including the design of reactors and their shielding.

\section{Historical}

Some experimental work on this problem, using the van de Graaff generator at the Argonne National Laboratory as a neutron source, was reported early in 1952 . $^{2}$ This work showed the feasibility of the method being employed but indicated that refinements in technique were required for extensive or accurate work in this field. The apparatus and results described in this report stem from the development initiated at that time.

$$
\text { Apparatus - (See illustrations, p. } 6 \text { \& } 7 \text { ) }
$$

The primary neutron source was a target bombarded by a beam of ions from the van de Graaff generator; in the work reported here neutrons were obtained from the $\mathrm{Li}^{7}(\mathrm{p}, \mathrm{n}) \mathrm{Be}^{7}$ reaction.

The neutron-producing source was placed in the cavity in a tank filled with borated water This tank had a collimating tube from the cavity to the exterior of the tank, permitting a beam of neutrons to emerge from the source target so as to impinge on a thin flat plate of scattering material.

Five neutron detectors were placed in a circular array around the scattering material. Thus scattering data were determined simultaneously at five angles.

${ }^{1}$ See, for example, Feshbach, Porter, and Weiskopf, Phys. Rev. 96 , 448 (1954).

${ }^{2}$ ANL-4798, pp. 5-18 and earlier reports. 


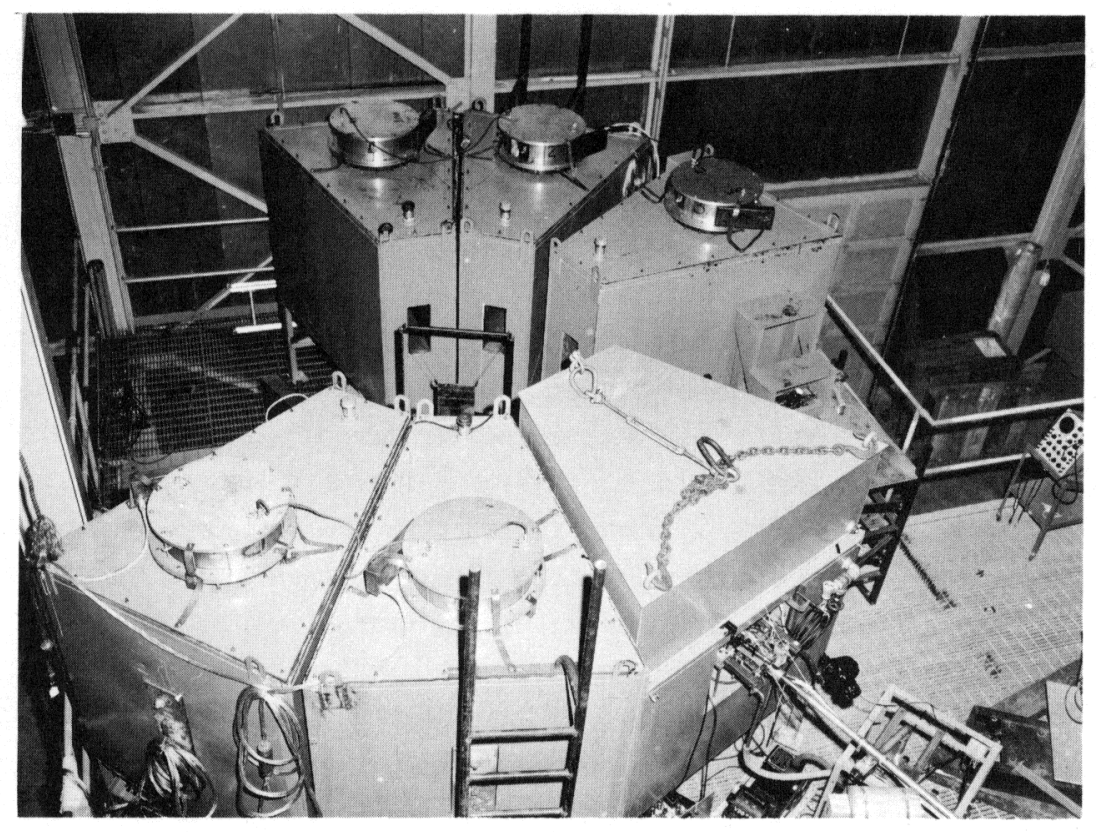

Completed apparatus shown with proton beam line entering source shield tank from lower right. Plate scatterer in open frame can be seen in center.

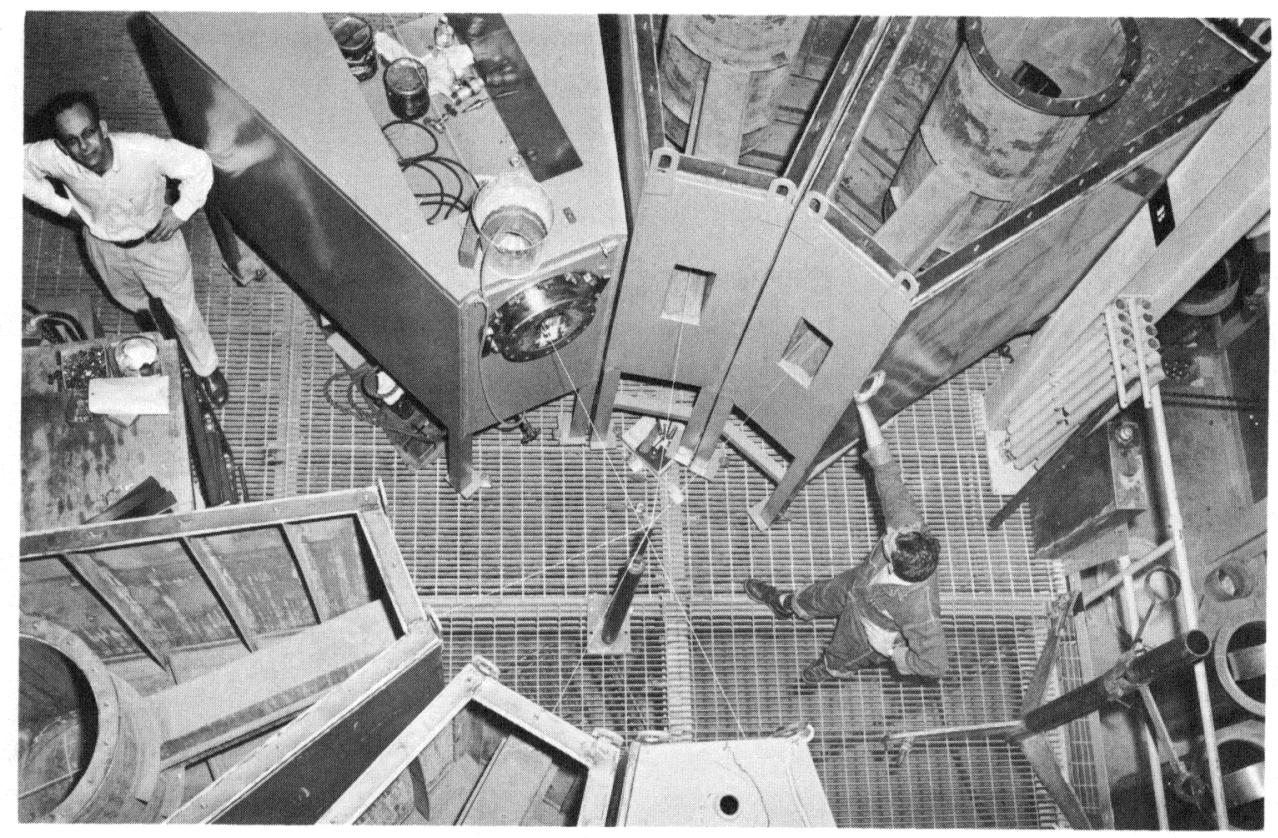

A view of apparatus in an early stage of assembly. Neutron source shield tank appears in the upper left. Each of the five detector tanks is aimed at scatterer position in center. 

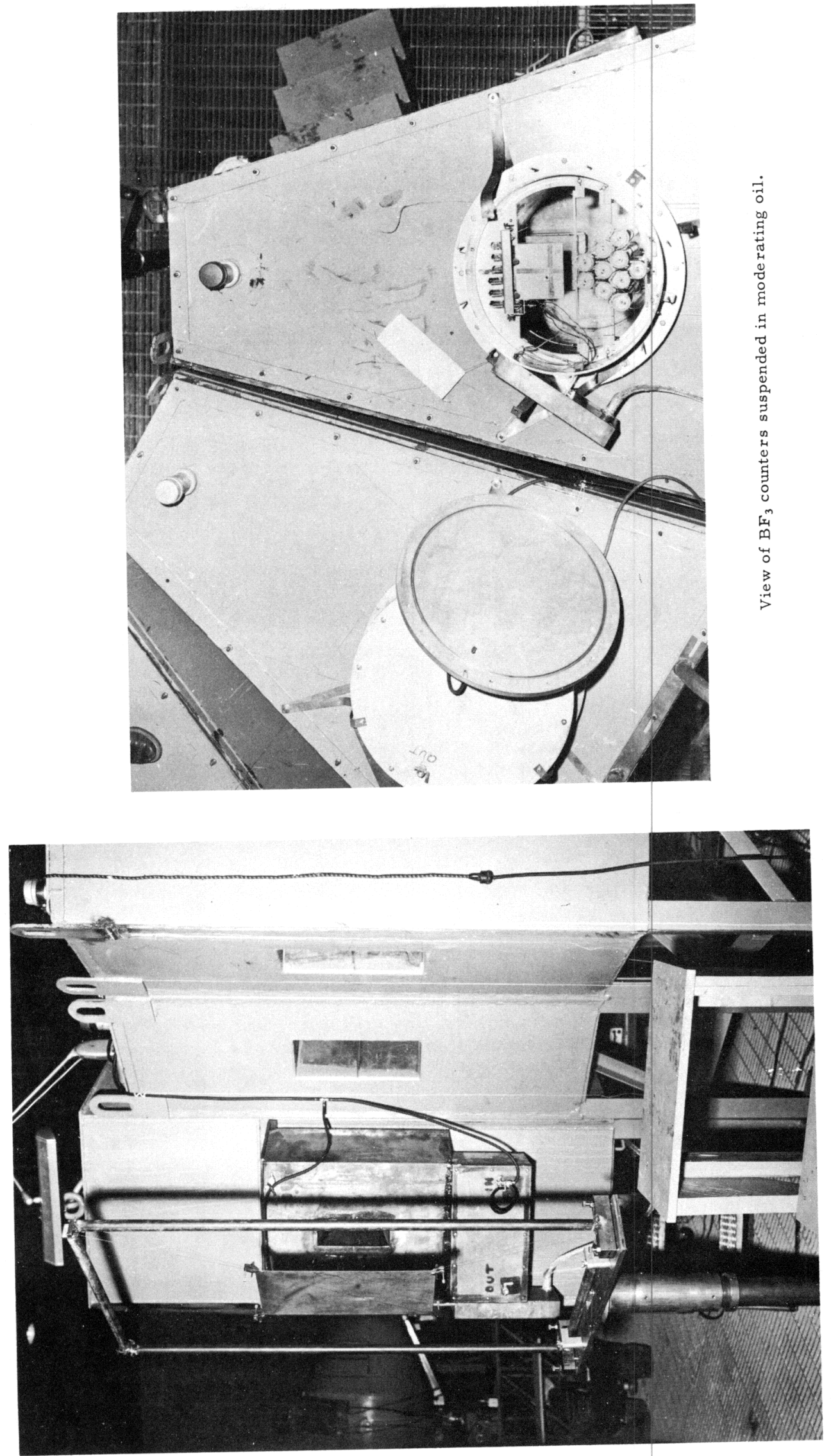
Each neutron detector contained ten $\mathrm{BF}_{3}$ proportional counters, 1.5 in. diameter, 20 in. active length. These were immersed in a neutron moderator of mineral oil in an aluminum tank $20 \mathrm{in}$. diameter by $4 \mathrm{ft}$ high. Each aluminum tank was placed inside a steel tank containing borated water. A collimator hole permitted neutrons from the scattere r to reach the counter assembly.

The dimensions of the entire array were such that the designed distance from the neutron source to the center of the scatterer was $5 \mathrm{ft}$; scatterer-to-detector distance, $7 \mathrm{ft}$; illuminated area of scatterer measured normal to the axis of the incident beam, 6 in. wide by 16 in. high; effective detector area, also $6 \mathrm{in.}$ wide by $16 \mathrm{in}$. high. The water tanks were in the form of truncated pie-shaped wedges (right-isoscelestrapezoidal cylinders) which fit together side by side. The five detector shield tanks each subtended 30 degrees in the horizontal plane at the center of the scatterer and the source shield tank subtended 40 degrees. The water shielding thus filled all the available space. Its adequacy was tested by plugging the collimator hole of one unit. Then the counting rate with 1-Mev neutrons being produced was less than 1/2000 of that with the collimator open. This shielding was entirely adequate at $1 \mathrm{Mev}$ and probably will be adequate up to or above $3 \mathrm{Mev}$.

The detector shield tanks each contained about 700 gallons of (borated) water; the source shield tank contained about 1000 gallons. The source shield tank had a removable lid, permitting reasonably simple access for servicing the lithium target.

Since $\mathrm{BF}_{3}$ counters in a moderator detect neutrons of all energies, the apparatus measured total scattering rather than just elastic scattering. It was therefore desirable that the efficiency of the detectors be independent of incident neutron energy so that if inelastic scattering were present, the measured cross section would not be a complicated function of the amount and type of inelastic scattering. Various arrays of the ten counters in each assembly were tried, until an array was obtained such that the response was within 10\% of that of a "long counter" (which was presumed to have a "flat" response) from $0.1 \mathrm{Mev}$ to $1.5 \mathrm{Mev}$. It is considered that the present degree of uniformity of response is adequate for these initial experiments.

By tests with neutron sources (photo-sources of $\mathrm{Sb}-\mathrm{Be}, \mathrm{Na}-\mathrm{Be}$, $\mathrm{Na}-\mathrm{D}_{2} \mathrm{O}$, and mixed $\left.\mathrm{Ra}(\alpha)-\mathrm{Be}\right)$ the five counters were found to have equal efficiencies within about 2 percent. (The data were of course corrected to uniform efficiency.) The efficiency for counting the neutrons actually impinging on the $6 \mathrm{in.} \mathrm{by} 16 \mathrm{in}$. face of each detector was between 12 percent and 15 percent; this was about three times the minimum estimate design figure used in planning the construction. 
The counting rates were such that with a lithium target of 10-kilovolt stopping power as a neutron source (which has a mean yield of about $10^{6}$ neutrons per steradian at $0^{\circ}$ per microcoulomb of protons), about 300 counts perminute per detector were obtained with a 15-microampere beam.

In most neutron scattering apparatus, adequately precise monitoring of the flux incident on the scatterer is very difficult. By measuring five angles at once, monitoring becomes somewhat less important. However, quite satisfactory monitoring is in fact obtained by locating two $\mathrm{BF}_{3}$ counters in the source shield tank assembly, close to the collimator hole. In this geometry, even with very thick scatterers, backscattering perturbs the monitor a negligible amount (less than $1 \%$ for a scatterer over a mean free path thick).

The background counting rate (the counting rate in the absence of a scattering sample) in this apparatus is predominantly due to air. Each detector, in addition to "seeing" the scattering sample, also "sees" a column of air about $2 \mathrm{ft}$ long which is "illuminated" by the primary neutron beam. This amount of air scatters about $1 \%$ of the neutrons. Thus background rates were predicted to be, and indeed were found to be, on the average, about $10 \%$ of the rate due to the scatterer. For some angles with some samples, the background was as great as $30 \%$. The air scattering could be reduced by building a large vacuum tank; however, such a step is not believed to be important at the present time.

The scattering measurements reported here were made with the five neutron detectors placed at fixed positions. The scatter-angles between the axis of the incident beam and the axes of the five counters were: $22^{\circ} 18^{\prime}, 54^{\circ} 4^{\prime}, 91^{\circ} 40^{\prime}, 112^{\circ} 25^{\prime}$, and $143^{\circ} 54^{\prime}$. For the se nominal angles, but with the extended sizes of scatterers and detectors employed, the mean angles of scatter and root mean square deviations from the mean have been calculated. (For further discussion on mean scattering angles see pp. 50 and 54.) These mean angles, again in order of increasing angle, were: $26.5^{\circ} \pm 5.4^{\circ}, 55.4^{\circ} \pm 5.0^{\circ}, 91.2^{\circ} \pm 5.6^{\circ}, 112.8^{\circ} \pm 2.4^{\circ}$ and $143.7^{\circ} \pm 2.4^{\circ}$. These calculations were made for the geometrical conditions of the experiment, including the fact that the scattering samples were mounted vertically, with the incident beam at an angle of $45^{\circ}$ to the normal to the plate surface. The first three detectors were placed to observe neutrons scattered from the side of the plate opposite that from which the beam was incident (that is, in a transmission-side geometry). The last two detectors (those at $112^{\circ}$ and $144^{\circ}$ ) observed neutrons from the same side from which the beam was incident on the plate (that is, in a reflection-side geometry). It will be noted that the transmission geometry had twice as large a rootmean-square spread in angle as the reflection geometry; this is a characteristic geometrical difference between the two cases. It should also be pointed out that this oblique orientation of the scatterer plate was a practical compromise chosen to minimize the difficulties in correcting for plural 
scattering. It is clear that nearly all neutrons will be plurally scattered away from any direction which is nearly in the plane of the plate. With the orientation chosen, no data were taken at an angle of emergence from the plate greater than $47^{\circ}$, measured from the normal to the plate surface. Thus we have been able to control the magnitude of the plural scattering perturbations so that in the most extreme cases that have been calculated, the corrections to any initial (uncorrected) differential scattering cross sections were never over about $35 \%$ and were usually a good deal less than this.

Most of the factors in the geometry of the system necessary to make highly refined analyses of the angular data can be determined by auxiliary experiments. Many of these will be quite practical in the near future when the five detectors, which have been fixed in location, will be mounted on a track so that they pivot about the location of the scattering sample. A few of the geometrical factors have been partially studied already. In particular, the collimation of the emergent beam from the source collimator seems to be excellent, at least up to $1.8 \mathrm{Mev}$. A good deal of the sharpness of definition of the beam may be ascribed to the use of an accurately rectangular, pyramidally tapered source collimator, with the source spot of neutrons (1/4 in. diameter spot) at the apex. The actual shielding material started 6 in. from the source spot and extended a distance of 28 in.

Another factor which was studied was the extent of background neutron counting which might be ascribed to neutrons already scattered by a scattering sample, which were then rescattered by air or shielding materials visible to a given detector. This effect was observed with the aid of a sodium-beryllium photoneutron source placed at or near the usual scatterer position, with and without a shadow-cone shield interposed between the source and given detector. In this manner an upper limit of $2 \%$ was placed on the amount of such scattering relative to the direct flux from the source. Of this $2 \%$, roughly half was produced by air scattering and half by other materials (located as an oppositely located shield tank would be). No corrections for this phenomenon have been made in the data as presented in this report. In a few cases, this effect could be a correction of some importance, but adequate information is not yet at hand to know just how to make the correction. 
SECTION II.

ANALYSIS OF THE DATA

\section{Introduction}

In order to present the data in a practical and useful form, it was decided to represent the differential cross section in the laboratory coordinate system by an expansion in a series of Legendre polynomials. It is then possible to obtain a compact and easily visualized description of the angular distribution over a range of incident neutron energies by graphing these series coefficients as a function of incident neutron energy.

In brief, the scheme of calculation involves reducing the actual neutron counting information to a representation in terms of Legendre polynomials. The coefficients in this representation are then used to carry through a correction of the observed counting information for plural scattering by an analytic theory of double scattering in plates. Then by iteration a new set of Legendre polynomial coefficients may be obtained. Reiteration may be employed until the answer has converged as closely as may be desired to a stable answer. A reiteration is necessary in very few cases.

In this scheme, one may see that the Legendre polynomials are required in the Laboratory coordinate system in order to complete the calculations. This representation is also useful for all practical problems in neutron transport. For use in nuclear theory, a representation in the center-of-mass coordinate system would be more desirable, It was decided not to delay issuing the data by trying to carry through the transformation, which would be very time consuming for the amount of data presented in this report.

In this analysis it is convenient to make several assumptions. These are:

(1) Since the resolution used in obtaining these data is approximately $100 \mathrm{kev}$, the quantity measured is very nearly

$$
\overline{\sigma(E, \Theta)}=\frac{1}{\Delta} \int_{\Delta} I(E) \sigma(E, \Theta) d E,
$$

where $I(E)$ is the spectrum of incident neutrons and $\Delta$ is their resolution width. Thus, all sharp resonance variation in the cross section is averaged out. It is assumed that all effects, such as "beam hardening, "energy loss on scattering, etc., which cause a variation in $I(E)$ or $\Delta$ can be neglected in the interpretation of $\sigma(E, \Theta)$. In the following we shall omit the bar in designating this cross section. 
(2) All polarization effects are neglected, i.e., $\sigma(\Theta)$ is not considered to be a function of the azimuthal angle, $\phi$.

(3) In the experiment a plane parallel scattering sample of finite thickness, $h$, is used (in practice $0<\mathrm{h}<0.5$ and usually $0.05<\mathrm{h}<0.10$; in units of mean free path). In order to correct the data for multiple scattering, it is assumed that the scatterer is infinite in the plane axis directions. It is further assumed that the scattering sample is "illuminated" by a parallel beam of neutrons and that the scattered neutrons are detected by a counter system which subtends a small solid angle at the scattering plate. The actual geometry, of course, cannot quite match these assumptions.

(4) As a consequence of assumptions (1) and (2), we do not distinguish elastic from inelastic scattering. We assume that the neutron detector measures all scattered neutrons with equal efficiency, a situation which is very nearly achieved in practice.

(5) Neutron capture is assumed to be negligible relative to neutron scattering; thus

$$
\sigma_{\mathrm{st}} / \sigma_{\mathrm{t}} \simeq 1
$$

where $\sigma_{\text {st }}$ denotes the total scattering cross section and $\sigma_{t}$ the total cross section. For almost every material measured in this survey

$$
0.9<\sigma_{s t} / \sigma_{t} \leqslant 1.0
$$

within the energy range in which measurements were made. In this discussion all charged particle reactions such as $(n, p)$, etc., are to be lumped with neutron capture.

(6) Finally, all multiple neutron producing reactions such as $(n, 2 n),(n$, fission), etc., are assumed to be negligible.

Calculation of the Legendre Polynomial Coefficients

We define the following symbols:

$\Phi_{0}$, the flux incident on the scattering sample, a flat plate. In the following discussion we use "flux" in the sense of neutrons per unit area integrated over a specified time.

A, the area, measured perpendicular to the direction of $\Phi_{0}$, of the sample "illuminated" by the incident flux. 
$\mathrm{N}_{\mathrm{Z}}$, the thickness, in atoms $/ \mathrm{cm}^{2}$, of the scattering sample, $Z$, measured in the direction normal to the plane of the plate.

$\theta_{0}$, the angle included by the directions of $\Phi_{0}$ and the normal to the scattering plate.

$\sigma_{t Z}$, the total neutron cross section in barns for the sample
$Z$. $\sigma_{\mathrm{Z}}\left(\mu_{\mathrm{m}}\right)$, the differential neutron scattering cross section, in barns per steradian, for the sample $\mathrm{Z}$ evaluated at the scattering angle whose cosine is $\mu \mathrm{m}$.

Thus, the total flux incident on the scattering sample is $A_{0} \Phi_{0}$ The fraction of this which is transmitted is

$$
A \Phi_{0} e^{-\alpha N_{Z}{ }^{\sigma} \mathrm{tZ}}
$$

where $\alpha \mathrm{N}_{\mathrm{Z}} \equiv \mathrm{N}_{\mathrm{Z}} / \cos \theta_{0}$ is the thickness of the scatterer in the direction of the incident beam. The fraction of the incident flux which is not transmitted, and which, therefore, has interacted, is

$$
A \Phi_{0}\left(1-e^{-\alpha N_{Z}{ }^{\sigma}} t Z\right) \text {. }
$$

Of this interacting flux, the fraction $\sigma_{\mathrm{Z}}\left(\mu_{\mathrm{m}}\right) / \sigma_{\mathrm{tZ}}$ is scattered into a unit solid angle at the angle whose cosine is $\mu_{\mathrm{m}}$. Thus the flux scattered into a detector which subtends the solid angle $\Delta \Omega_{\mathrm{m}}$ is

$$
\Delta \Omega_{\mathrm{m}} \frac{\sigma_{\mathrm{Z}}\left(\mu_{\mathrm{m}}\right)}{\sigma_{\mathrm{tZ}}} \mathrm{A} \Phi_{0}\left(1-\mathrm{e}^{-\alpha \mathrm{N}_{\mathrm{Z}} \sigma_{\mathrm{tZ}}}\right) .
$$

If the efficiency of this detector is $\epsilon_{\mathrm{m}}$, the number of counts it records, corrected for background, is

$$
\epsilon_{\mathrm{m}} \Delta \Omega \mathrm{m} A \Phi_{0}\left(1-\mathrm{e}^{\left.-\alpha \mathrm{N}_{\mathrm{Z}} \sigma_{\mathrm{tZ}}\right)} \frac{\sigma_{\mathrm{Z}}\left(\mu_{\mathrm{m}}\right)}{\sigma_{\mathrm{tZ}}}\right. \text {. }
$$

The counts are measured with several separate detectors set up at the various angles identified by the index $\underline{m}$. These counters have slightly differing values of $\epsilon_{\mathrm{m}}$ and $\Delta \Omega_{\mathrm{m}}$. By a separate measurement 
using a rotating neutron source (e.g., $\mathrm{Sb}-\mathrm{Be}, \mathrm{Na}-\mathrm{Be}$, and $\mathrm{Na}-\mathrm{D}_{2} \mathrm{O}$ photoneutron sources and a Ra-Be source), it is possible to determine the relative efficiency of each counter. Therefore, a set of correction factors, $\rho_{\mathrm{m}}$, can be determined such that the product $\rho_{\mathrm{m}} \epsilon_{\mathrm{m}} \Delta \Omega_{\mathrm{m}}$ is the same for all angles measured. The counting rate, corrected to uniform efficiency, is then

$\rho_{\mathrm{m}} \in \underset{\mathrm{m}}{\Delta \Omega_{\mathrm{m}}} \mathrm{A}^{\Phi_{0}}\left(1-\mathrm{e}^{-\alpha \mathrm{N}_{\mathrm{Z}} \sigma_{\mathrm{tZ}}}\right) \frac{\sigma_{\mathrm{Z}}(\mu \mathrm{m})}{\sigma_{\mathrm{tZ}}}=\mathrm{k}\left(1-\mathrm{e}^{-\alpha \mathrm{N}_{\mathrm{Z}}{ }^{\sigma} \mathrm{tZ}}\right) \frac{\sigma_{\mathrm{Z}}\left(\mu_{\mathrm{m}}\right)}{\sigma_{\mathrm{tZ}}}$,

where

$$
\mathrm{k}=\rho_{\mathrm{m}} \epsilon_{\mathrm{m}} \Delta \Omega_{\mathrm{m}} \mathrm{A} \Phi_{0}
$$

is a constant independent of $\mathrm{m}$.

It is clear that the flux, $A \Phi_{0}$, is the same for all the angles measured simultaneously. However, it is further assumed that a constant value of this incident flux can be maintained by normalizing all counts to a standard count as measured by a monitor whose count is proportional to the forward neutron output of the $\operatorname{Li}(\mathrm{p}, \mathrm{n})$ source.

In brief, for the counter at the scattering angle $\cos ^{-1} \mu_{\mathrm{m}}$ :

$\mathrm{g}\left(\mu_{\mathrm{m}}\right)$ is the observed gross counts for a particular scatterer.

$\mathrm{m}$ is the monitor count observed simultaneously.

$\mathrm{G}\left(\mu_{\mathrm{m}}\right)=\frac{\mathrm{g}\left(\mu_{\mathrm{m}}\right)}{\mathrm{m}} \quad \begin{aligned} & \text { is then the gross count normalized to a unit monitor } \\ & \text { reading. }\end{aligned}$

$\mathrm{b}\left(\mu_{\mathrm{m}}\right)$ is the observed background count.

$\mathrm{b}\left(\mu_{\mathrm{m}}\right)$ is the simultaneously observed monitor reading.

$\mathrm{B}\left(\mu_{\mathrm{m}}\right)=\frac{\mathrm{b}\left(\mu_{\mathrm{m}}\right)}{\mathrm{m}}$ is then the normalized background count. 
Thus

$$
\mathrm{G}\left(\mu_{\mathrm{m}}\right)-\mathrm{B}\left(\mu_{\mathrm{m}}\right)
$$

is the normalized net count at $\cos ^{-1} \mu_{\mathrm{m}}$ due to the scattering sample.

Defining the corrected and normalized count to be

$$
\mathrm{C}_{\mathrm{Z}}\left(\mu_{\mathrm{m}}\right)=\rho_{\mathrm{m}}\left[\mathrm{G}\left(\mu_{\mathrm{m}}\right)-\mathrm{B}\left(\mu_{\mathrm{m}}\right)\right] \text {, }
$$

for the scatterer Z, we obtain approximately the quantity defined by Eq. (1) so that as a first approximation

$$
\mathrm{C}_{\mathrm{Z}}\left(\mu_{\mathrm{m}}\right)=\mathrm{k}\left(1-\mathrm{e}^{-\alpha \mathrm{N}_{\mathrm{Z}}{ }^{\sigma} \mathrm{tZ}}\right) \frac{\sigma_{\mathrm{Z}}\left(\mu_{\mathrm{m}}\right)}{\sigma_{\mathrm{tZ}}} \text {, }
$$

where $\mathrm{k}$ is a constant which is yet to be determined.

Next the differential cross section is expanded in a series of Legendre polynomials as

$$
\sigma\left(\mu_{\mathrm{m}}\right)=\sum_{\ell=0}^{\mathrm{N}-1} \mathrm{a}_{\ell} \mathrm{P}_{\ell}\left(\mu_{\mathrm{m}}\right) \quad, \quad \mathrm{m}=1, \ldots \mathrm{N}
$$

where we have omitted the subscript, $Z$, which identifies a particular scattering sample. It is as sumed that $\mathrm{N}$ is sufficiently large that the coefficients a $l$ are negligibly small for all $l \geqslant N$. A discussion of this assumption is given in the following section of this report.

The evaluation of such an expansion presupposes, of course, that measurements of the scattering are made for at least $\mathrm{N}$ different values
of the scattering angle. Regarding the polynomials $\mathrm{P}_{\ell}\left(\mu_{\mathrm{m}}\right)$ as the elements of a square matrix, it is possible to introduce a reciprocal matrix whose elements, $\mathrm{p}_{\mathrm{mn}}$, are defined by

$$
\sum_{\mathrm{m}=1}^{\mathrm{N}} \mathrm{P}_{\ell}\left(\mu_{\mathrm{m}}\right) \mathrm{p}_{\mathrm{mn}}=\delta_{\ell \mathrm{n}}
$$


Then

$$
\sum_{\mathrm{m}=1}^{\mathrm{N}} \sigma\left(\mu_{\mathrm{m}}\right) \mathrm{p}_{\mathrm{mn}}=\sum_{\mathrm{m}=1}^{\mathrm{N}} \sum_{\ell=0}^{\mathrm{N}-1} \mathrm{a}_{\ell} \mathrm{P}_{\ell}\left(\mu_{\mathrm{m}}\right) \mathrm{p}_{\mathrm{mn}}=\mathrm{a}_{\mathrm{n}},
$$

or

$$
a_{\ell}=\sum_{m=1}^{N} \sigma\left(\mu_{m}\right) p_{m l}
$$

The total scattering cross section, $\sigma_{\text {st }}$, for the element $Z$ is defined by the integral

$$
\sigma_{\mathrm{stz}}=\int \sigma_{\mathrm{z}}(\mu) \mathrm{d} \Omega=\int_{0}^{2 \pi} \mathrm{d} \phi \int_{-1}^{1} \mathrm{~d} \mu \sigma_{\mathrm{Z}}(\mu)=4 \pi \mathrm{a}_{0},
$$

where we have used Eq. (3) and the orthogonality of the polynomials $\mathbf{P} \ell(\mu)$. Combining this result with Eq. (5), we have

$$
\mathrm{a}_{0}=\frac{\sigma_{\mathrm{stZ}}}{4 \pi}=\sum_{\mathrm{m}=1}^{\mathrm{N}} \sigma_{\mathrm{Z}}\left(\mu_{\mathrm{m}}\right) \mathrm{p}_{\mathrm{mo}}
$$

From Eq. (2) we now obtain

$$
\begin{aligned}
& \sum_{m=1}^{N} C_{Z}\left(\mu_{m}\right) p_{m o}=k \frac{\left(1-e^{-\alpha N_{2}{ }^{\sigma} t Z}\right)}{\sigma_{Z}} \sum_{m=1}^{N}{ }^{\sigma} z^{\left(\mu_{m}\right) p_{m o}}= \\
& \frac{\mathrm{k} \sigma_{\mathrm{stZ}}}{4 \pi \sigma_{\mathrm{tZ}}}\left(1-\mathrm{e}^{-\alpha \mathrm{N}_{\mathrm{Z}}{ }^{\sigma} \mathrm{tZ}}\right)
\end{aligned}
$$

Equation (7) can be used to evaluate the constant $\mathrm{k}$ by assuming that measurements have been made with a target for which $\sigma_{t Z}$ is known and $\sigma_{\mathrm{stZ}}=\sigma_{\mathrm{tZ}} \cdot$ For neutron energies below $2 \mathrm{Mev}$, the best material for this purpose is carbon; it exhibits no inelastic scattering, no resonance structure, es sentially no capture, and its differential cross section is not very far from isotropic in this energy region. Replacing the symbol $\mathrm{Z}$ by $C$ to denote a carbon scatterer and considering the value of $\sigma_{\text {stC }}=\sigma_{t C}$ as known from independent transmission measurements, it is possible to
calculate the value of the constant 


$$
\mathrm{k}=\frac{4 \pi}{1-\mathrm{e}^{-\alpha \mathrm{N}_{\mathrm{C}}{ }_{\mathrm{tC}} \mathrm{N}}} \sum_{\mathrm{m}=1}^{\mathrm{N}} \mathrm{C}_{\mathrm{C}}\left(\mu_{\mathrm{m}}\right) \mathrm{p}_{\mathrm{mo}} .
$$

Once $\mathrm{k}$ is determined, we use Eq. (7) to obtain for any scattering sample other than carbon

$$
\frac{\sigma_{\mathrm{stZ}}}{\sigma_{\mathrm{tZ}}}=\frac{4 \pi}{\mathrm{k}} \frac{\sum_{\mathrm{m}=1}^{\mathrm{N}} \mathrm{C}_{\mathrm{Z}}\left(\mu_{\mathrm{m}}\right) \mathrm{p}_{\mathrm{mo}}}{1-\mathrm{e}^{-\alpha \mathrm{N}_{\mathrm{Z}}{ }_{\mathrm{tZ}}}} .
$$

If we define a quantity $\delta$ by

$$
\sigma_{\mathrm{tZ}}=(1+\delta) \sigma_{\text {stZ }} \quad(\delta<1),
$$

and solve the se equations simultaneously for $\sigma_{\text {stZ }}$, we obtain

$$
\sigma_{\mathrm{stZ}}=-\frac{1}{\alpha \mathrm{N}_{\mathrm{Z}}(1+\delta)} \ln \left\{1-\frac{4 \pi}{\mathrm{k}}(1+\delta) \sum_{\mathrm{m}=1}^{\mathrm{N}} \mathrm{C}_{\mathrm{Z}}\left(\mu_{\mathrm{m}}\right) \mathrm{p}_{\mathrm{mo}}\right\} .
$$

For scattering elements, $Z$, whose capture cross section is zero, $\delta=0$, and Eq. (9) becomes

$$
\sigma_{\text {st }}=-\frac{1}{\alpha N_{Z}} \ln \left\{1-\frac{4 \pi}{k} \sum_{m=1}^{N} C_{Z}\left(\mu_{m}\right) p_{m o}\right\} .
$$

As long as the scattering sample is not too thick, the total scattering cross section can be obtained from Eq. (10) quite accurately even for $\delta \simeq 0.1$. In these measurements we choose scattering samples of such thickness that the quantity

$$
\mathrm{g}=\frac{4 \pi}{\mathrm{k}} \sum_{\mathrm{m}=1}^{\mathrm{N}} \mathrm{C}_{\mathrm{Z}}\left(\mu_{\mathrm{m}}\right) \mathrm{p}_{\mathrm{mo}}
$$

is of the order of 0.1 . Only in exceptional cases will g exceed a maximum of 0.3 . For $\delta=0.1$ and $g=0.3$, the error in using Eq. (10), instead of Eq. (9), is $1.7 \%$. Normally $\mathrm{g} \simeq 0.1$; then if $\delta=0.1$ the error is $0.7 \%$. Since $\delta<<0.1$ in most cases, the error in calculating the total scattering cross section from Eq. (10) is negligibly small. All of the calculations which we have made are based on Eq. (10). 
Combining Eqs. (2) and (7) we obtain

$$
\sigma_{\mathrm{Z}}\left(\mu_{\mathrm{m}}\right)=\frac{\mathrm{C}_{\mathrm{Z}}\left(\mu_{\mathrm{m}}\right) \sigma_{\mathrm{stz}}}{4 \pi \sum_{\mathrm{m}=1}^{\mathrm{N}} \mathrm{C}_{\mathrm{Z}}\left(\mu_{\mathrm{m}}\right) \mathrm{p}_{\mathrm{mo}}}
$$

Once the $N$ quantities $\sigma_{\mathrm{Z}}\left(\mu_{\mathrm{m}}\right)$ are determined by Eqs. (10) and (11), the coefficients $a_{\ell}$ can be determined from Eqs. (5) and (6). expansion

The representation of the results of this survey is given by the

$$
\sigma_{Z}(\mu)=\frac{\sigma_{\text {stZ }}}{4 \pi} \sum_{\ell=0}^{N-1} \omega_{\ell} P_{\ell}(\mu),
$$

where $\omega_{0}=1$. There are two reasons why such a representation was chosen: 1) In the correction for multiple scattering the coefficients $\omega_{l}$ enter naturally. 2) The coefficients $\omega l$ are independent of any normalization to a known total cross section: This may be shown by combining
Eqs. (5), (6) and (11) to obtain

$$
\omega_{\ell}=\frac{\sum_{m=1}^{N} C_{Z}\left(\mu_{m}\right) p_{m \ell}}{\sum_{m=1}^{N} C_{Z}\left(\mu_{m}\right) p_{m o}} .
$$

From Eq. (5) it may be noted that the quantities $\mathrm{p}_{\mathrm{m} \ell}$ serve to weight the individual measurements, $\sigma_{\mathrm{Z}}\left(\mu_{\mathrm{m}}\right)$, in the determination of $\mathrm{a}_{\ell}$. It is,therefore, of some practical interest to choose the angles at which measurements are made in such a manner that the se matrix elements, $\mathrm{P}_{\mathrm{m} \ell}$, are all of comparable magnitude.

Termination of the Legendre Series

As was mentioned in the previous section, the maximum value of $\mathrm{N}$ in the series,

$$
\sigma(\mu)=\sum_{\ell=0}^{N-1} a_{\ell} P_{\ell}(\mu),
$$


is determined by the number of angles at which measurements are made. In order that thi's series be a valid representation of $\sigma(\mu)$, at angles other than the measured $\cos ^{-1} \mu_{\mathrm{m}}$, it is necessary that $a_{l}$ vanish for $l \geqslant N$.

There are, of course, good theoretical grounds for believing that only a finite number of terms contribute appreciably to the series (12). The termination of this series corresponds to neglecting the contribution of all partial waves whose orbital angular momentum is greater than $(\mathrm{N}+1) / 2$. For this reason, we have consistently made measurements at an odd number of angles.

Furthermore, there are several methods of checking the validity of the expansion (12). These include:

(1) The values of the total scattering cross sections, $\sigma$ stZ' calculated from Eq. (10) agree with known values of $\sigma_{\mathrm{tZ}}$, the total cross section, determined by previous transmission measurements. For those elements where absorption is known to be negligible, the calculated $\sigma$ st $\mathrm{Z}$ usually has been well within $10 \%$ of the independently measured $\sigma_{t Z}$ even though we have made measurements at only five scattering angles thus far. At the present stage of the experiments this is as good agreement as could be expected for a number of reasons.

(2) The values of $a_{3}$ and $a_{4}$ remain small compared with $a_{1}$ and $a_{2}$ for lower neutron energies and lighter scattering elements where this is to be expected.

(3) The results for scattering by hydrogen, where the angular distribution is known very well, came out as predicted.

(4) The scattering by elements previously measured elsewhere agrees with our results not only at the angles we measured, but also at interpolated angles using the series (12).

(5) There exists a general smoothness and self-consistency of the results for most materials over the energy range which was measured.

(6) At low enough energy, the scattering is expected to be isotropic in the center-of-mass system. For the lightest elements this leads to a considerable anisotropy in the laboratory system which should be exhibited by a non-zero value for $a_{1}$ and much smaller values of $a_{2}, a_{3}$ and $a_{4}$. Finding this predicted behavior is a further check on the reliability of this representation of the data. 
It is, of course, possible to obtain expressions for the effect of higher order non-vanishing coefficients on the coefficients, al, in the series (12) with $N=5$. Let the true differential cross section be given as

$$
\sigma(\mu)=\sum_{\ell=0}^{\infty} \mathbf{A}_{\ell} \mathbf{P}_{\ell}(\mu)
$$

and compare the coefficients, $a_{\ell}$, as calculated using an expansion

$$
\sigma(\mu)=\sum_{\ell=0}^{4} a_{\ell} \mathbf{P}_{\ell}(\mu),
$$

with the true coefficients, $A_{\ell}$. The results are

$$
\begin{aligned}
& a_{0}=A_{0}-.020 A_{5}-.016 A_{6}+\ldots \\
& a_{1}=A_{1}-.075 A_{5}-.005 A_{6}+\ldots \\
& a_{2}=A_{2}+.261 A_{5}+.218 A_{6}+\ldots \\
& a_{3}=A_{3}-.115 A_{5}-.060 A_{6}+\ldots \\
& a_{4}=A_{4}+.267 A_{5}-.083 A_{6}+\ldots . .
\end{aligned}
$$

It is evident from these expressions* that for any reasonable behaviour of the higher $A_{\ell}$, the total cross section, $4 \pi a_{0}$, and the integral

$$
\int \sigma(\mu)(1-\mu) \mathrm{d} \Omega=4 \pi\left(\mathrm{A}_{0}-\frac{1}{3} \mathrm{~A}_{1}\right)
$$

which occurs in the definition of the transport cross section are essentially independent of the higher order coefficients.

For the heavier elements at high neutron energy, there remains some question about the adequacy of the expansion (12) with $N=5$. In the future the above scheme of calculation will be generalized to give a most probable set of coefficients, $a \ell$, in the sense of a least squares analysis. We plan to extend these measurements to probably nine angles, spaced at about equal increments in degrees between the minimum practical angle $\left(\sim 20^{\circ}\right)$ and the maximum $\left(145^{\circ}\right)$. This will allow a meaningful least squares analysis for all coefficients up to $a_{7}$. One advantage of this type of analysis is that it provides a measure of the probable error for each $a_{\ell}$ depending, essentially, on how accurately the calculated curve, $\sigma_{Z}(\mu)$, can be fitted to the individual measured values $\sigma_{\mathrm{Z}}\left(\mu_{\mathrm{m}}\right)$.

*The expressions relating the $\mathrm{a}_{\ell}$ and $\mathrm{A}_{\ell}$ are explicitly for the angles actually used in the experiment. 


\section{Multiple Scattering Corrections}

In the previous sections, we have shown how a representation of angular distribution data is obtained in terms of a series of Legendre polynomials. We consider now the problem of correcting the approximate answers so obtained for the effects of multiple scattering.

We introduce the following notation:

$$
a=\alpha^{-1}=\cos \theta_{0} ; \phi_{0}=0 \quad \text { defines the direction of the incident }
$$
neutron beam.

$c=\gamma^{-1}=\cos \theta_{2} ; \phi_{2}$ defines the direction of the emergent beam.

$\Theta$ is the scattering angle: $\quad \mu=\cos \Theta=a c+\sqrt{\left(1-a^{2}\right)\left(1-c^{2}\right)} \cos \phi_{2}$.

$$
\begin{aligned}
& \mathrm{h}_{\mathrm{Z}}=\mathrm{N}_{\mathrm{Z}} \sigma_{\mathrm{tZ}} \quad \text { is the normal thickness of the scat- } \\
& \text { tering sample, } \mathrm{Z} \text {, in units of mean } \\
& \text { free path. } \\
& \Phi_{1}(\mathrm{~h}, \mathrm{a}, \mathrm{c}) \quad \text { is the emergent singly scattered flux } \\
& \text { per unit solid angle (per unit incident } \\
& \text { flux) in the direction }\left(c, \phi_{2}\right) \text {. } \\
& \Phi_{2}(h, a, c) \text { is the corresponding emergent doubly } \\
& \text { scattered flux. } \\
& \Phi_{1}(\mathrm{~h}, \mathrm{a}) \text { is the total (integrated) emergent } \\
& \text { singly scattered flux. } \\
& \Phi_{2}(h, a) \quad \text { is the total emergent doubly scattered } \\
& \text { flux. }
\end{aligned}
$$

In a separate section on the theory of double scattering in thin plates (see p. 40), it is shown that

$$
\Phi_{1}(h, a, c)= \begin{cases}\alpha \frac{\sigma(\mu)}{\sigma_{t}} \frac{e^{-\alpha h}-e^{-\gamma h}}{\gamma-\alpha} & \operatorname{transmission}(\gamma \geq 0) \\ \alpha \frac{\sigma(\mu)}{\sigma_{t}} \frac{e^{(\gamma-\alpha) h}-1}{\gamma-\alpha} & \text { reflection }(\gamma \leq 0)\end{cases}
$$




$$
\Phi_{1}(\mathrm{~h}, \mathrm{a})=\sum_{\ell=0}^{\mathrm{N}-1} \omega_{\ell} \mathrm{T}_{\ell}(\mathrm{h}, \mathrm{a}) ; \omega_{\ell} \equiv \frac{\mathrm{a}_{\ell}}{\mathrm{a}_{0}},
$$

where the parameters $\mathrm{T}_{\ell}(\mathrm{h}, \mathrm{a})$ are given in graphical form (Fig. 1$)$ for $l=0,1, \ldots, 4 ; a=\cos 45^{\circ}$ and $0<h \leqslant .20$. Also

$$
\Phi_{2}(\mathrm{~h}, \mathrm{a}, \mathrm{c})=\sum_{\mathrm{k}=0}^{\mathrm{N}-1} \sum_{\ell=\mathrm{k}}^{\mathrm{N}-1} \omega_{\ell} \omega_{\mathrm{k}} \mathrm{Q}_{\ell \mathrm{k}}(\mathrm{h}, \mathrm{a}, \mathrm{c}),
$$

where the parameters $Q_{l k}(h, a, c)$ for $k=0,1, \ldots, 4$; $a=\cos 45^{\circ}$; $0<\mathrm{h} \leqslant .20$; and $\left(\theta_{2}, \phi_{2}\right)=\left(9^{\circ} 10^{\prime}, 180^{\circ}\right)\left(23^{\circ}, 0^{\circ}\right),\left(46^{\circ} 30^{\prime}, 180^{\circ}\right)$, $\left(157^{\circ} 30^{\prime}, 0^{\circ}\right)$ and $\left(170^{\circ} 50^{\prime}, 180^{\circ}\right)$ are given in Figs. 2, 3, 4, 5 and 6. Finally

$$
\Phi_{2}(h, a)=\sum_{k=0}^{N-1} \sum_{l=k}^{N-1} \omega_{l} \omega_{k} \Lambda_{\ell k}(h, a) .
$$

In the initial calculation, it was assumed that the number of counts observed by the counter at $\cos ^{-1} \mu_{\mathrm{m}}=\Theta_{\mathrm{m}}$ is given by Eq. (2)

$$
\mathrm{C}_{\mathrm{Z}}\left(\mu_{\mathrm{m}}\right)=\mathrm{k}\left(1-\mathrm{e}^{-\alpha \mathrm{h}_{\mathrm{Z}}}\right) \frac{\sigma_{\mathrm{Z}}\left(\mu_{\mathrm{m}}\right)}{\sigma_{\mathrm{tZ}}} \text {. }
$$

Actually, however, the observed count is given by

$$
k\left\{\sum_{j=1}^{\infty} \Phi_{j}\left(h_{z}, a, c_{m}\right)\right\}
$$

If we assume that the angular distribution of the sum of emergent particles scattered a number of times greater than two is (approximately) the same as that given by $\Phi_{2}(h, a, c)$, then the actual number of counts $S_{z}\left(\mu_{m}\right)$ recorded by the counter at $\Theta_{\mathrm{m}}$ is approximately

$$
\mathrm{S}_{\mathrm{Z}}\left(\mu_{\mathrm{m}}\right)=\mathrm{k}\left\{\Phi_{1}\left(\mathrm{~h}_{\mathrm{Z}}, \mathrm{a}, \mathrm{c}_{\mathrm{m}}\right)+\eta \Phi_{2}\left(\mathrm{~h}_{\mathrm{Z}}, \mathrm{a}, \mathrm{c}_{\mathrm{m}}\right)\right\} \text {, }
$$

where

$$
\eta=\frac{1-e^{-\alpha h_{z}}-\Phi_{1}\left(h_{Z}, a\right)}{\Phi_{2}\left(h_{Z}, a\right)}
$$




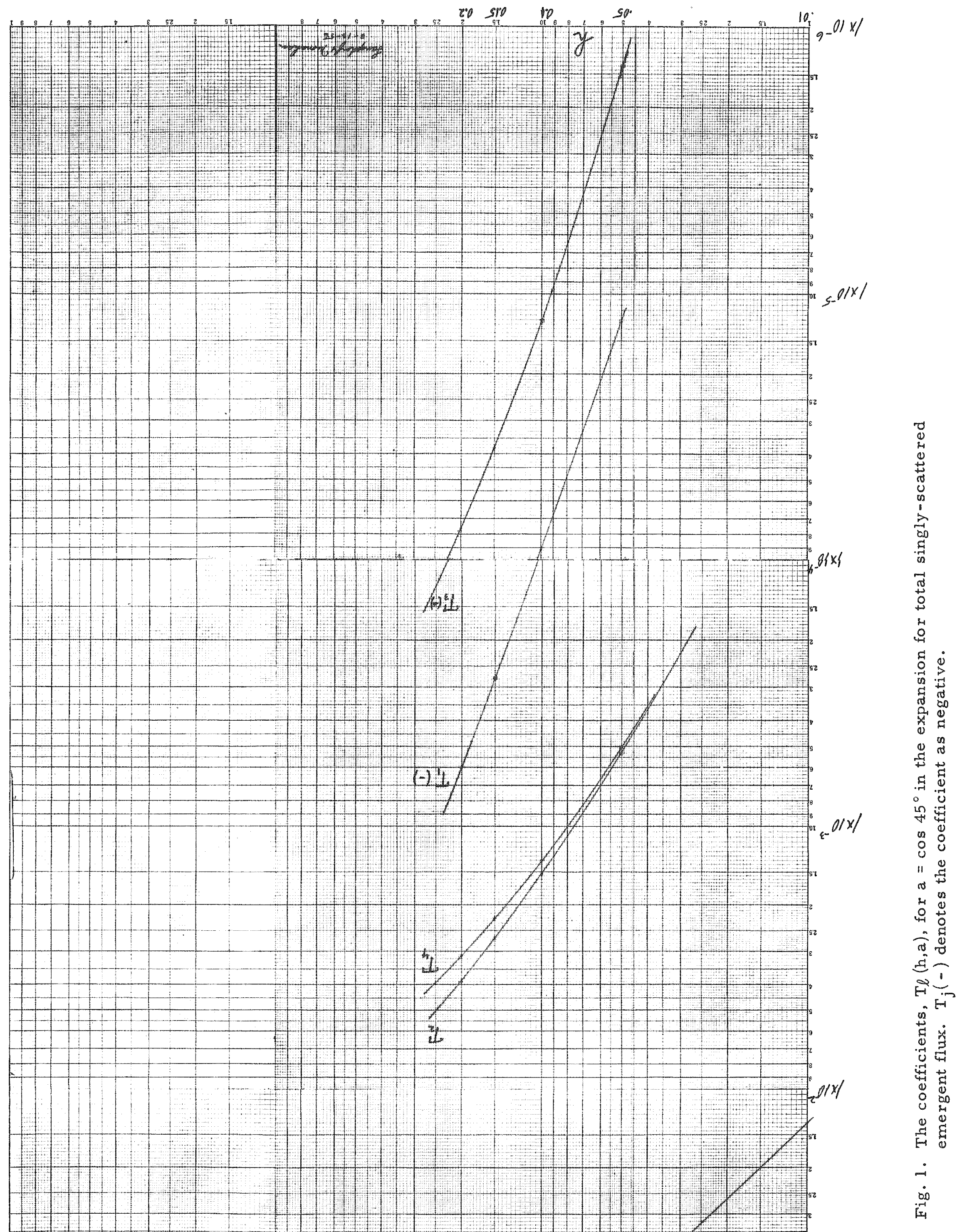




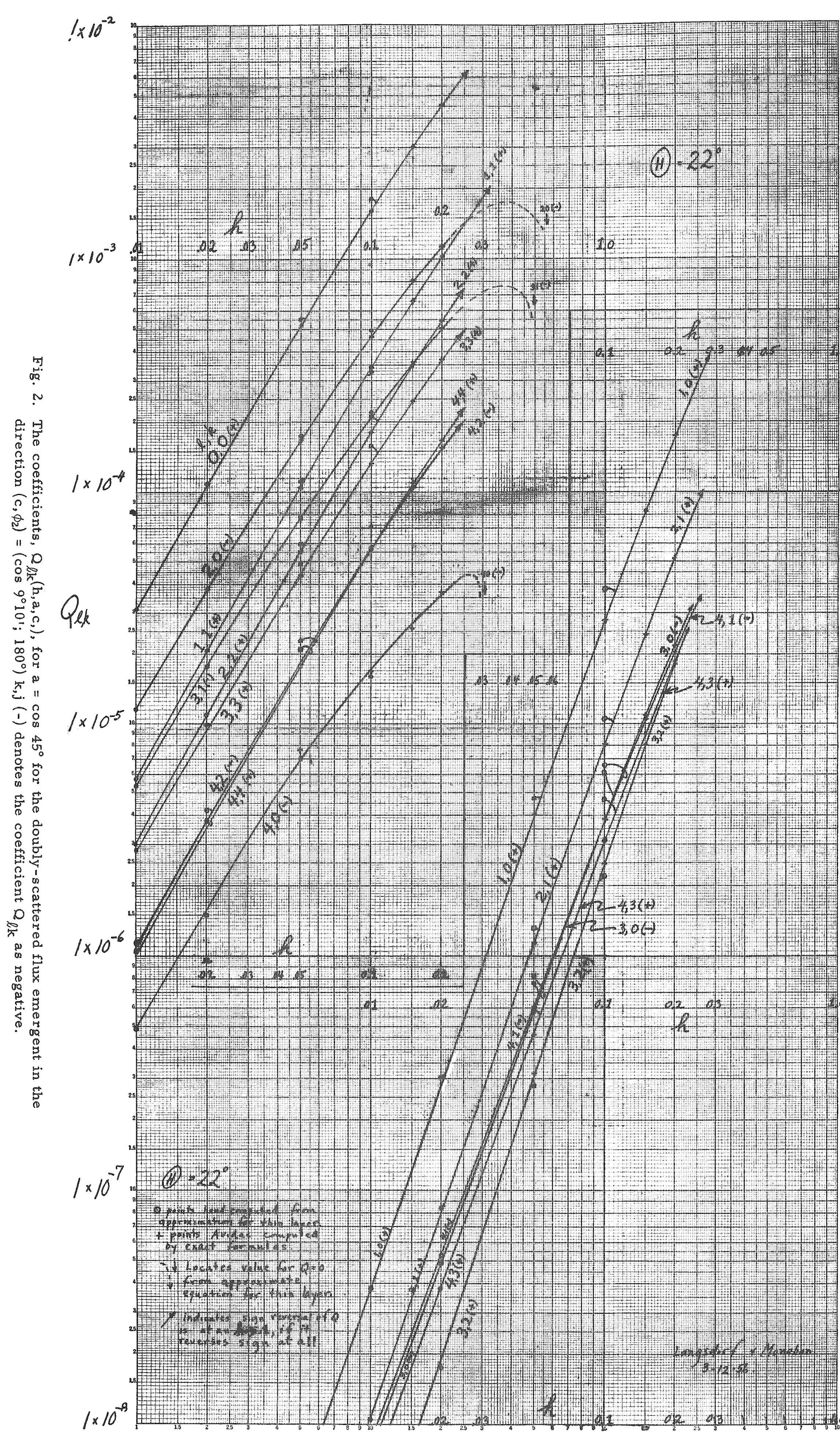




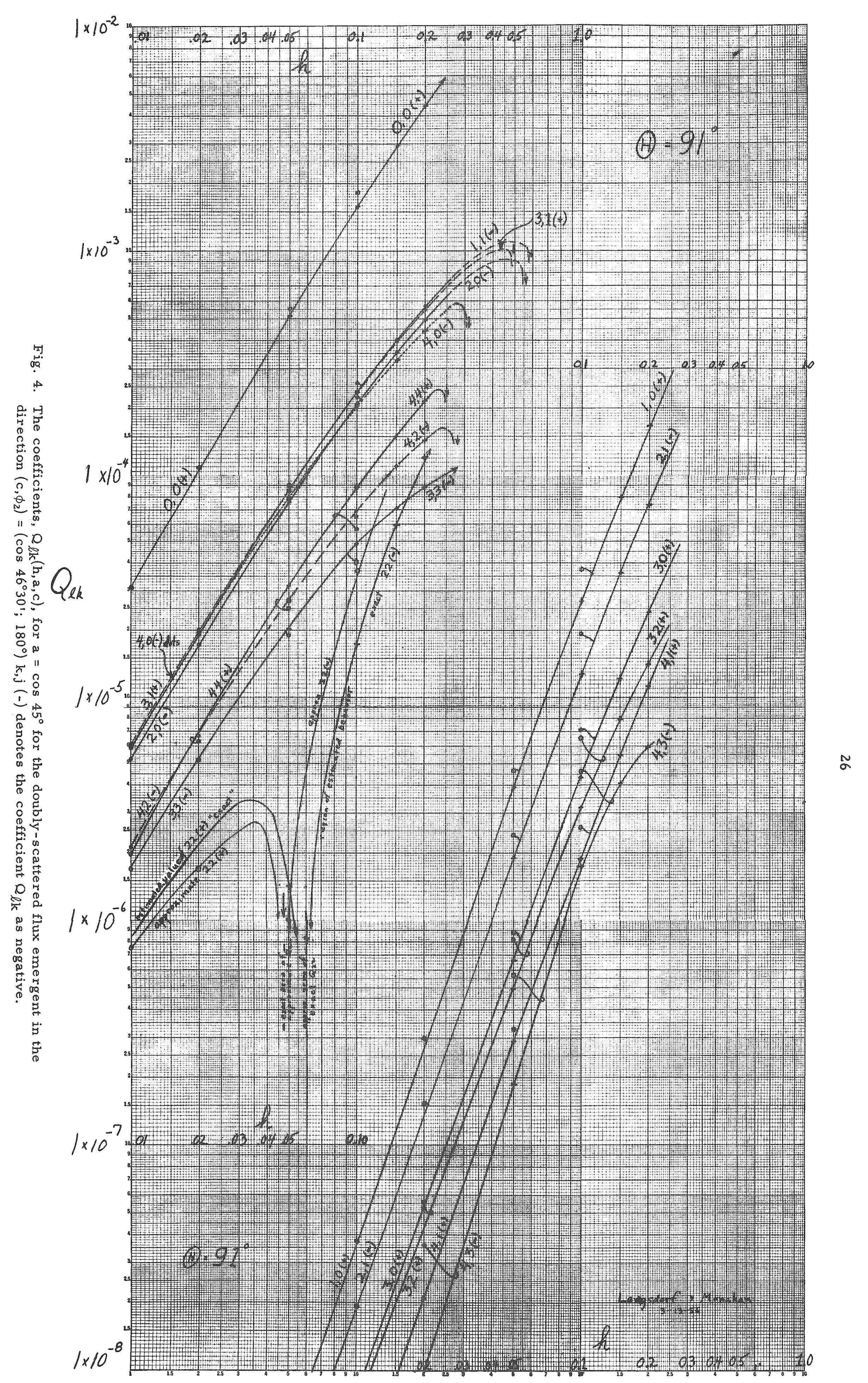


ก

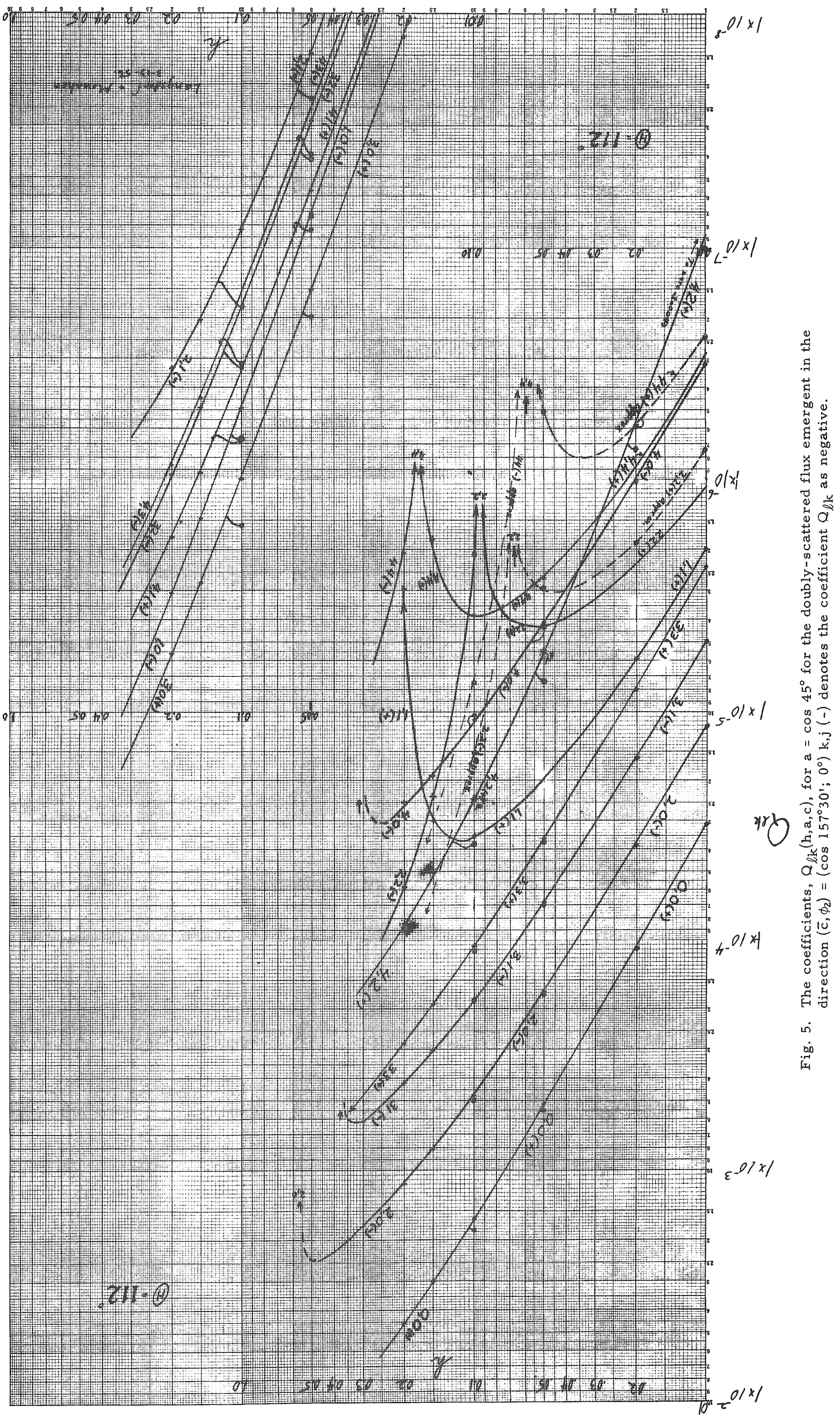




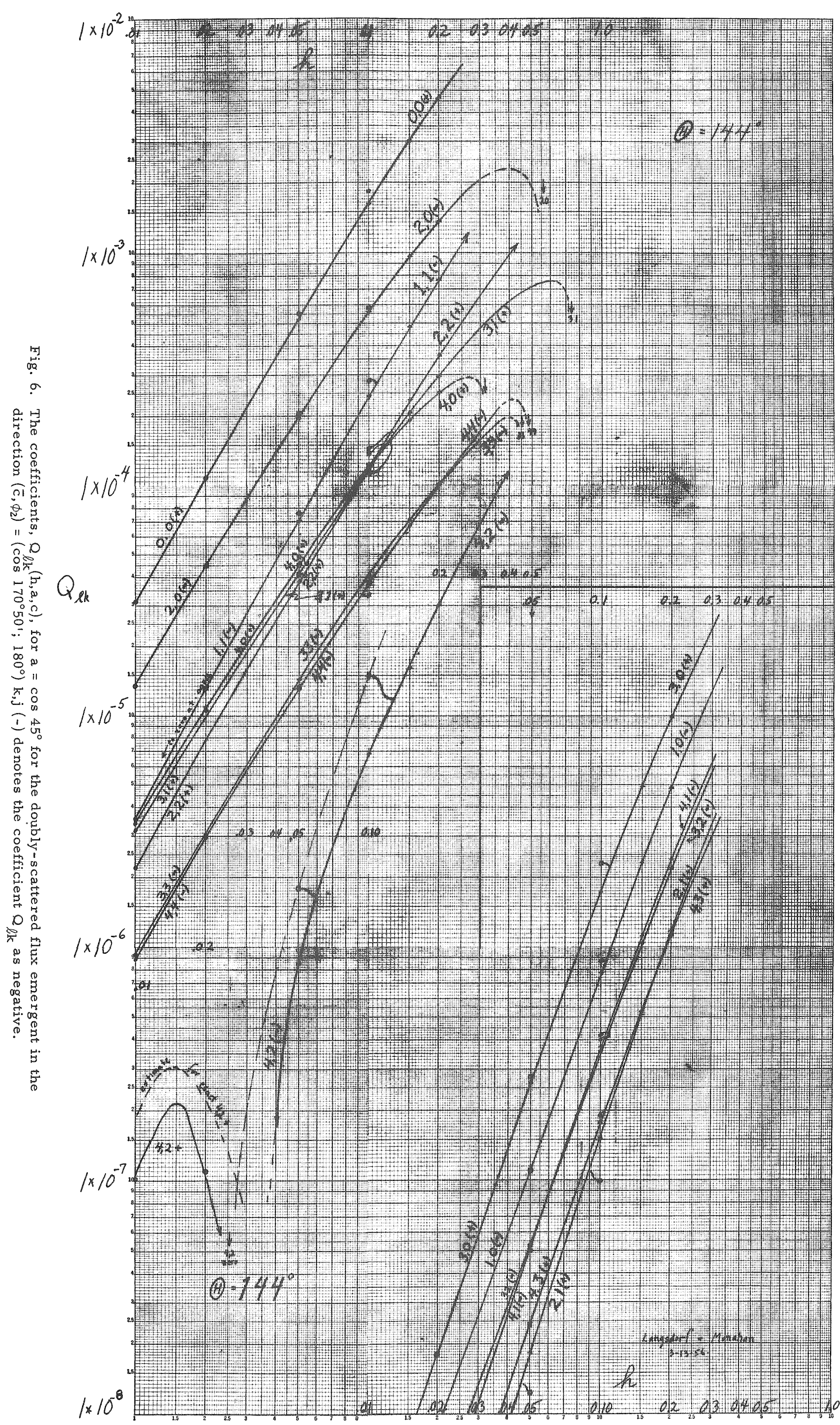


The labor in calculating the functions $\Lambda_{\ell \mathrm{k}}$ was found to be so great that it was not carried through. Therefore we did not employ Eq. (19) to calculate $\eta$. Instead a somewhat less exact $\eta$ was calculated by assuming that

$$
\frac{\Phi_{m+1}(h, a)}{\Phi_{m}(h, a)}=a \text { constant independent of } m \text {, }
$$

i.e., we assume that the ratio of the integrated flux due to successive orders of scattering is independent of the order of scatter. It can then be shown that

$$
\eta=\frac{1-\mathrm{e}^{-\alpha h}-\Phi_{1}(\mathrm{~h}, \mathrm{a})}{\Phi_{2}(\mathrm{~h}, \mathrm{a})} \simeq \frac{1-\mathrm{e}^{-\alpha \mathrm{h}}}{\Phi_{1}(\mathrm{~h}, \mathrm{a})}
$$

We may now calculate, using Eq. (18) and Eq. (20),

$$
C_{Z}\left(\mu_{m}\right)=S_{Z}\left(\mu_{m}\right)\left\{\frac{\sigma_{Z}\left(\mu_{m}\right)\left(1-e^{-\alpha h_{Z}}\right)}{\sigma_{t Z}\left[\Phi_{1}\left(h_{Z}, a, c_{m}\right)+\eta \Phi_{2}\left(h_{Z}, a, c_{m}\right)\right]}\right\}
$$

where

$$
\mathrm{S}_{\mathrm{Z}}\left(\mu_{\mathrm{m}}\right)=\rho_{\mathrm{m}}\left[\mathrm{G}_{\mathbf{i}}\left(\mu_{\mathrm{m}}\right)-\mathbf{B}_{\mathbf{j}}\left(\mu_{\mathrm{m}}\right)\right],
$$

and $\mathrm{C}_{\mathrm{Z}}\left(\mu_{\mathrm{m}}\right)$, the observed counts corrected for multiple scattering, as defined by Eq. (20), is the quantity described by Eq. (2).

The bracketed quantity on the right hand side of Eq. (21) is, of course, evaluated using the first approximate, uncorrected values of $\sigma_{\mathrm{Z}}\left(\mu_{\mathrm{m}}\right), \mathrm{h}_{\mathrm{Z}}$, and $\mathrm{a}_{\ell}$ or $\omega_{\ell}$. The corrected values, $\mathrm{C}_{\mathrm{Z}}\left(\mu_{\mathrm{m}}\right)$, etc., so obtained can then be resubstituted into Eqs. (5), (10), and (11) of the previous sections to obtain corrected values for the coefficients a $l$, the total scattering cross section $\sigma_{\text {stz }}$ ( and the equivalent corrected $h_{Z}$ ), and the differential cross section $\sigma_{\mathrm{z}}\left(\mu_{\mathrm{m}}\right)$. It may be worth while to point out that this correction must be carried out simultaneously for the carbon standard. This means that the constant, $\mathrm{k}$, of Eq. (8) must be recomputed using for $\mathrm{C}_{\mathrm{C}}\left(\mu_{\mathrm{m}}\right)$ the counting rate for carbon as corrected by Eq. (21). This process is repeated until a consistent set of values of the $a_{l}{ }^{\prime}$ is obtained. 
The calculations of the uncorrected $\sigma_{\text {st }}$ and $\omega_{l}$ were carried through in accordance with the system formulated in the sections above for all the data as shown on the graphs in this report. The corrections made by the system here described were carried through on only a fraction of the data. The detailed explanations for reading the corrected data are given on page 53, and in the figure titles of the graphs.

To determine the consistency achieved after correcting results by this system of calculation, a few materials were measured with a series of samples of various thicknesses. The results of these calculations are shown on Fig. (7) and Fig. (8), with detailed explanations in the figure titles. It is clear from these figures (as a little thought about the geometrical conditions of the experimental arrangements will also make obvious) that as one increases the scattering sample thickness, the increase in plural scattering at the angles we measured is usually to be expected to be greater than the decrease in singly scattered neutrons reaching the detector. Therefore the uncorrected values of $\sigma_{\text {st }}$ and $\sigma_{\mathrm{Z}}\left(\mu_{\mathrm{m}}\right)$ are usually rising functions of thickness. Indeed, in over a hundred correction calculations, only two or three cases were found where the plural scattering corrected counts were greater than the uncorrected, and then only at the $22^{\circ}$ scattering angle. In fact, the only reason why corrected $\sigma_{\text {st }}$ and $\sigma_{Z}\left(\mu_{m}\right)$ etc., are ever increased in making corrections is that their values are relative to carbon, so that when the correction (downward) in carbon counts is greater than the correction (downward) in any other material, the relative corrections are such as to lead to an increase in $\sigma_{\text {stZ, etc. }}$

It is of interest to point out that the functions $Q_{\ell k}(h, a, c)$ are very rapidly varying functions of $h$, varying about as $h^{n}$ where $2<n<3$ usually. As a result, $\Phi_{2}(\mathrm{~h}, \mathrm{a}, \mathrm{c})$ in $\mathrm{Eq} .(15)$ is far more sensitive to the value of $h$, therefore to $\sigma_{s t Z}$, than to the values of the $\omega_{l}$. In consequence, it was found that most often iteration of our results to obtain corrected values was necessary almost exclusively in order to reach a suitably corrected $\sigma_{\mathrm{stZ}}$. Indeed, the uncorrected $\sigma_{\mathrm{stZ}}$ was often greater than the true $\sigma_{\mathrm{tZ}}$ (total cross section). Thus it has become clear that in future one should whenever posible measure the total cross section at nearly the same time as and for the same neutron spectrum as used for, the differential cross section. The measured $\sigma_{t Z}$ will then give an $h$ which is closer to a correct value to use in calculating $\Phi_{2}(\mathrm{~h}, \mathrm{a}, \mathrm{c})$ then when $\mathrm{h}$ is calculated from the uncorrected $\sigma_{\mathrm{stZ}} \cdot$ Furthermore, such an (independent) measure of $\sigma_{t Z}$ will give a good internal consistency check in the experiment. 


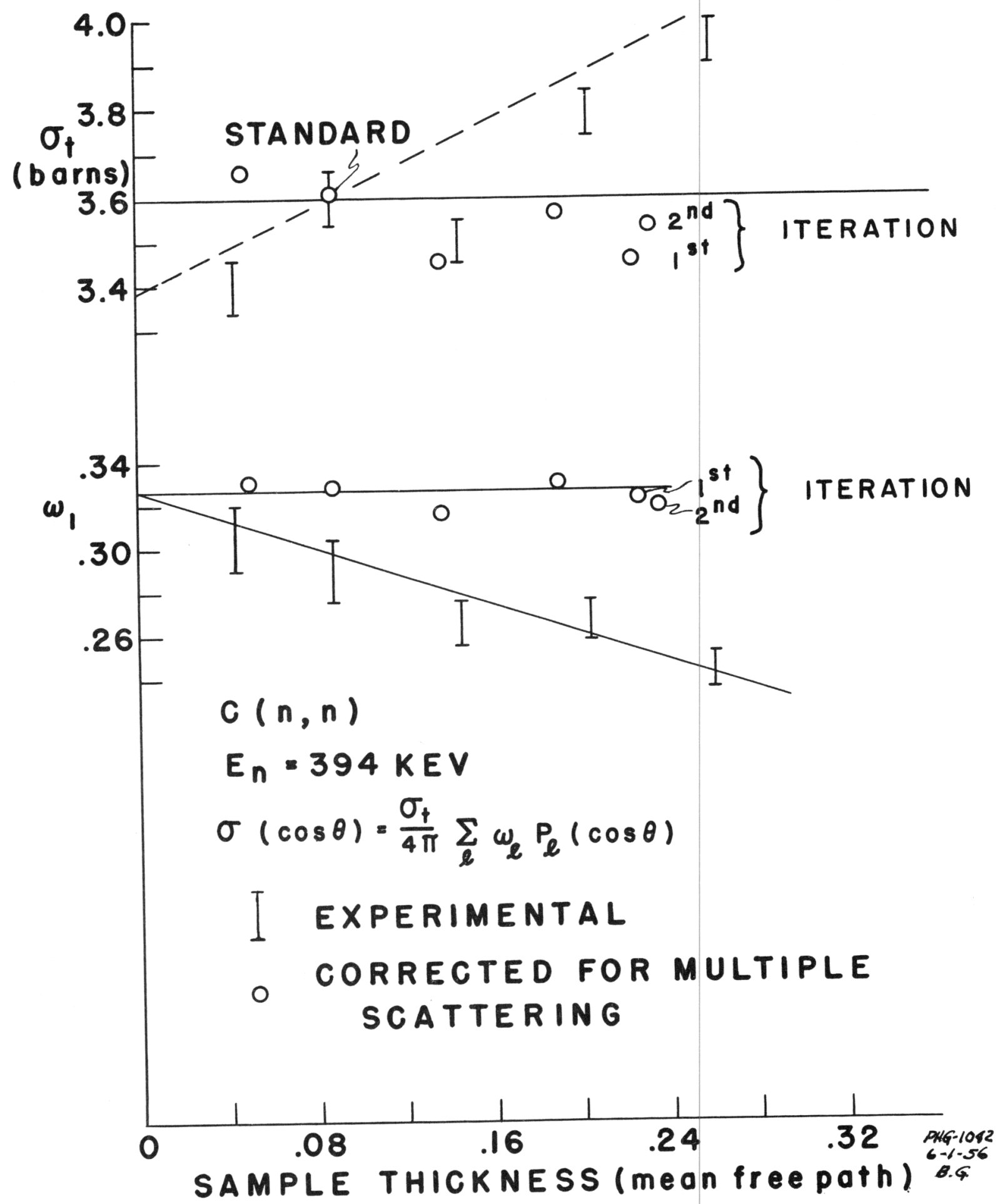

Fig. 7. The differential cross section of graphite at $394 \mathrm{Kev}$ as a function of sample thickness. The open circles indicate the same measurements corrected for plural scattering. 

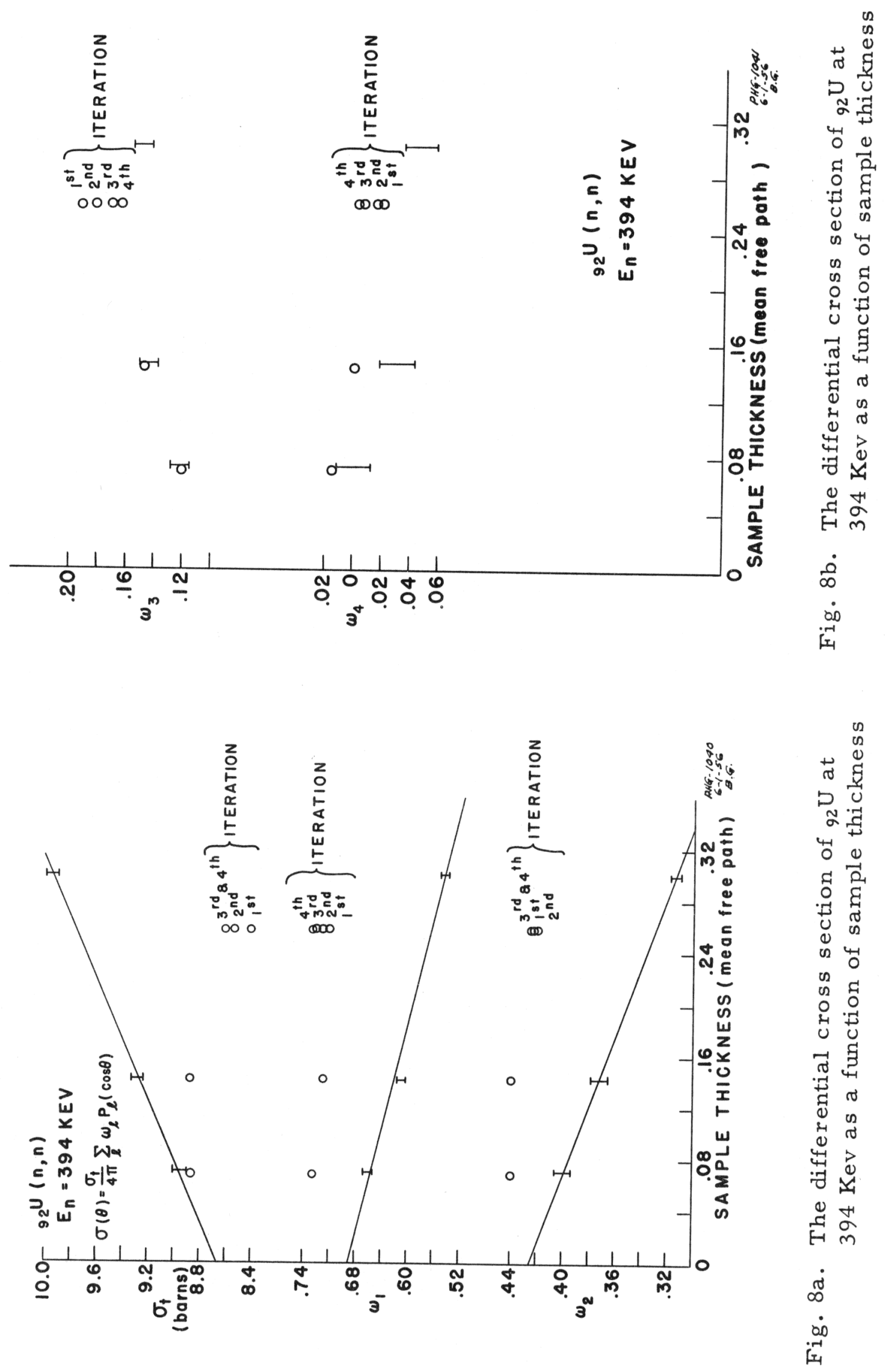


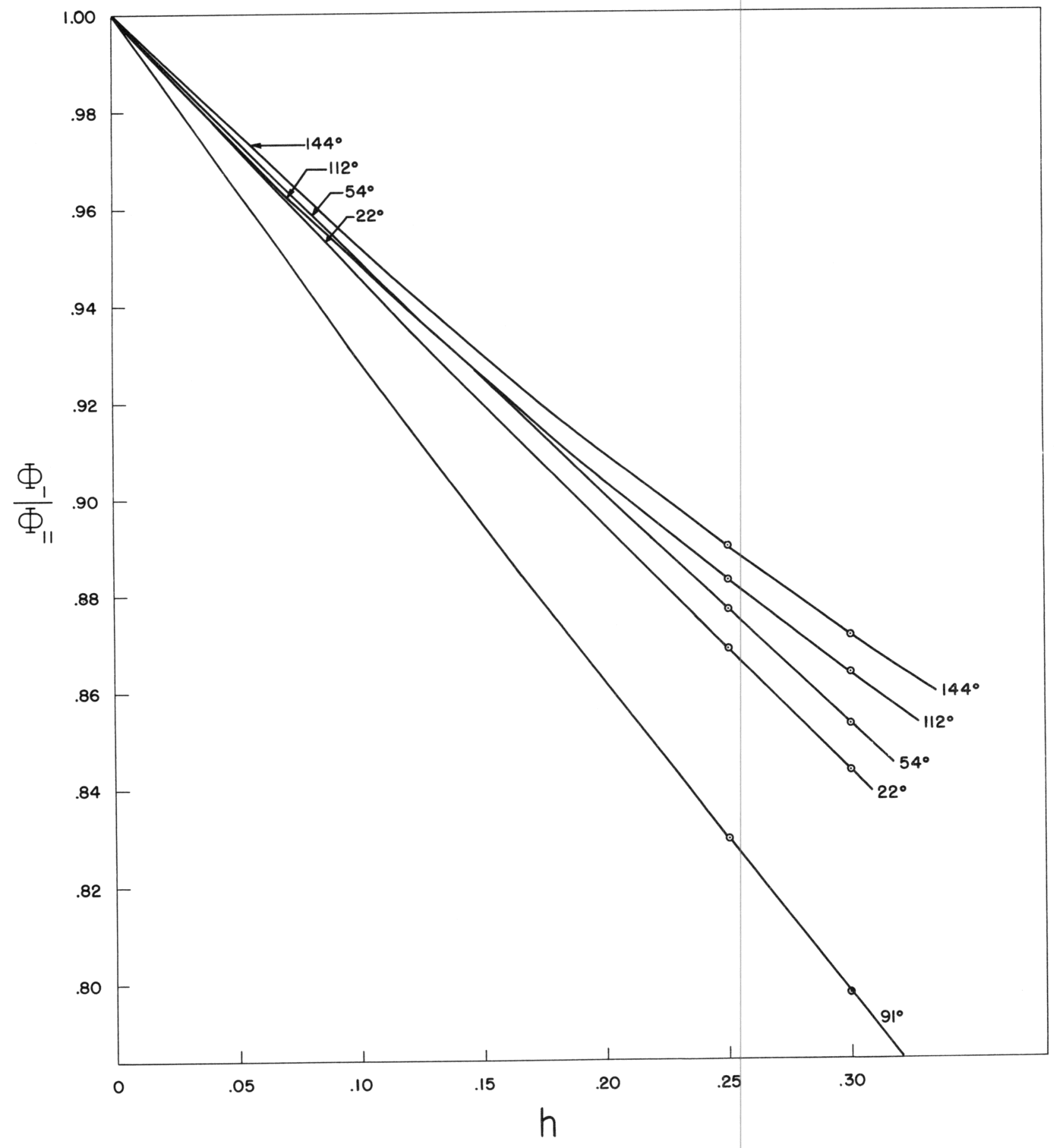

Fig. 9. The emergent singly scattered flux, $\Phi_{1}(\alpha, \gamma)$ per unit idealized scattered flux, $\Phi_{11}$, for flat plates, versus thickness, $h$, at the five scattering angles, $\Theta$, indicated. The incident flux is at an obliquity of $45^{\circ}=\sec ^{-1} \alpha$. 
The reason why literature values of $\sigma_{t z}$ (such as are to be found in BNL-325) were not used, was that in general the se were obtained for a different neutron spectrum, different sample thicknesses, and with unknown inaccuracies. Therefore such literature values would often have been more grossly in error than our own uncorrected $\sigma_{\text {st }}$. In some cases, there is no value of $\sigma_{t Z}$ available in the literature at present.

Discussion on the problem of an adequate determination of $\sigma_{t}$ by transmission measurements for use with the calculations of scattering data:

The scattering data is best determined using thin sheets, in the geometry of these experiments. We consider that the most desirable thickness is for $0.05 \leqslant \mathrm{~h} \leqslant 0.1$ where $\mathrm{h}=\mathrm{N}_{\mathrm{t}} \sigma_{\mathrm{t}}$. Then double scattering will not require a very large correction and triple and higher order scattering will be practically negligible.

In the experiments we have performed the flat plates of scatterer have been placed at an obliquity of $45^{\circ}$ to the neutron beam so that the transmission, $\mathrm{T}$, will be

$$
\mathrm{T}=\mathrm{e}^{-\sqrt{2} \mathrm{~h}}=\mathrm{e}^{-\sqrt{2} \mathrm{~N} \sigma_{\mathrm{t}}}
$$

Then we obtain

$$
\frac{d \sigma_{t}}{\sigma_{t}}=-\frac{d T}{\sqrt{2 h T}} \text {. }
$$

Therefore a fractional error $\frac{\Delta T}{T}$ is magnified by an amount $1 / \sqrt{2} \mathrm{~h}$ in determining the fractional error $\Delta \sigma_{t} / \sigma_{t} \cdot$ In the above range of $h$, the magnification is of the order of 7 to 14 . For example, if a million counts are obtained for "sample in" and "sample out" measurements to obtain $I$ and $I_{0}$ respectively, so that $I$ and $I_{0}$ are each statistically accurate to $0.1 \%$, the accuracy of $\mathrm{T}=\mathrm{I} / \mathrm{I}_{0}$ will be about $0.14 \%$. Then the per cent accuracy of $\sigma_{\mathrm{t}}$ in the range of $0.05 \leqslant h \leqslant 0.10$ will be $1 \%$ to $2 \%$.

Unless the counting system used for transmission is well adapted to counting of the order of a million counts in a reasonable time, and adequate flux for such an intensity is also available. some other expedient should be used. One possibility is to rotate 
the plate to such an oblique angle that the transmission is about $T=0.5$. The most serious difficulty with this scheme is that if the material being measured has resonances, whether resolved or not, beam hardening effects in such a thick layer may cause the measured $\sigma_{t}$ to be as much as $5 \%$ lower than its correct value for the scattering geometry. 
SECTION III.

\section{SINGLE AND DOUBLE SCATTERING IN PLATES}

\section{A. Introduction}

Our measurements of the angular distribution of neutrons scattered from flat plates indicate that the effect of multiple scattering can be appreciable (greater than $10 \%$ of single scattering) even for thick nesses less than 0.1 mean free path. Although there exibts an extensive literature on the subject of multiple scattering, little of it has been found to be applicable to the present situation in which a beam of neutrons is incident at an oblique direction on a plane-parallel scattering sample, which has rather strongly anisotropic scattering properties.

The work to be described in this section g1ves exact expressions for the emergent doubly scattered flux under the conditions described above. The assumptions used in the derivation of the se equations are those referred to in Section II. Although the assumption of thin plates does not enter formally in these derivations, this discussion is limited for all practical purposes to thicknesses for which the contribution of third and higher order processes to the total scattering is not very large.

\section{B. Notation and Conventions}

Consider a coordinate system oriented with respect to a scattering target as shown in the figure. The thickness of the target, measured along the Z-direction, i.e., normal to the target face, is denoted by h. This, as well as all other distances, is measured in units of mean free path. The lateral ( $X$ and $Y$ ) dimensions of the scattering sample are taken as essentially infinite compared with h.

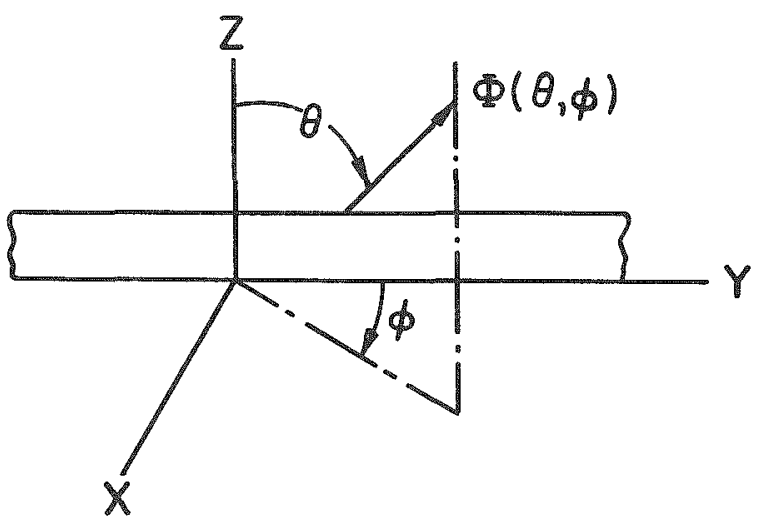

The incident flux in the direction $\left(a=\alpha^{-1}=\cos \theta_{0}, \phi_{0}\right)$ is taken as unity. Thus the singly scattered flux, $\Phi_{1}\left(b, \phi_{1}\right)$, emergent in the direction $\left(b=\beta^{-1}=\cos \theta_{1}, \phi_{1}\right)$ and the doubly scattered flux, $\Phi_{2}\left(c, \phi_{2}\right)$, emergent in the direction $\left(c=\gamma^{-1}=\cos \theta_{2}, \phi_{2}\right)$ are to be interpreted as flux per unit solid angle per unit incident flux. 
The scattering material is characterized by a phase function: $\mathrm{p}(\mathrm{a}, \mathrm{b})$, defined by

$$
p(a, b)=p_{a b}=p(\mu)=\frac{4 \pi \sigma(\mu)}{\sigma_{t}}=p(\Theta),
$$

where $\mu=\cos \Theta$ is the cosine of the scattering angle as ordinarily defined,

$$
\mu=a b+\sqrt{\left(1-a^{2}\right)\left(1-b^{2}\right)} \cos \left(\phi_{0}-\phi_{1}\right) \quad .
$$

$\sigma(\mu)$ is the differential cross section for scattering from the direction $\left(a, \phi_{0}\right)$ to the direction $\left(b, \phi_{1}\right) ; \sigma_{t}$ is the corresponding total scattering cross section. The notation, $\mathrm{p}_{\mathrm{ab}}$, for the phase function implies the corresponding azimuthal angles $\phi_{0}$ and $\phi_{1}$.

In order to fix the coordinate system uniquely with respect to directions which are physically well defined, we choose the $\phi_{0}=0$ plane as being defined by the direction of the $Z$-axis and the direction of the incident beam (i.e., the $Y-Z$ plane).

In the following treatment of double scattering it is neces sary to adopt a specific convention with regard to sign, particularly for the trigonometric functions. The positive $\mathrm{Z}$-direction will be defined as the direction pointing toward the scattering plate on the side from which the incident flux impinges. The cosines $a, b$, and $c$ together with the associated secants $\alpha, \beta$, and $\gamma$ will be considered as intrinsically positive. When a negative magnitude is required, it will be shown explicitly, either by writing, e.g., $-\alpha$ or $\bar{\alpha}$. The symbols $\bar{\alpha}$, etc., will only be used to indicate the direction of a ray which has a component in the negative $Z$ direction. The symbols $\frac{t}{a}$, etc., as arguments of a function shall imply that the functional relation is valid for both positive and negative directions of a ray. Length variables, $\tau, \lambda, \kappa$ and $h$ are taken as positive magnitudes.

Since the two cases are treated separately, we distinguish between transmitted and reflected flux by writing $\Phi(t)=\Phi(t)=\Phi(t)$ for the flux emergent on the transmission side of the scattering plate and $\Phi(-)=$ $\Phi(-)=\Phi(s)$ for the flux emergent on the reflection side.

The expansion

$$
\sigma(\mu)=\frac{\sigma t}{4 \pi} \sum_{l=0}^{N} \omega_{\ell} \mathrm{P}_{\ell}(\mu)
$$

has been discussed in the previous section of this report. Thus 
$\mathrm{p}_{\mathrm{ab}}=\sum_{\ell=0}^{N} \omega_{\ell} \mathrm{P}_{\ell}(\mu)=\sum_{\ell=0}^{N} \omega_{\ell} \sum_{\mathrm{m}=0}^{\ell} \frac{(\ell-\mathrm{m}) !}{(\ell+\mathrm{m}) !}\left(2-\hat{s}_{0} \mathrm{~m}\right) \cos \mathrm{m}\left(\phi_{1}-\phi_{0}\right) \mathrm{P}_{\ell}^{\mathrm{m}}(\mathrm{a}) \mathrm{P}_{\ell}^{\mathrm{m}}(\mathrm{b})$,

where we have made use of the addition theorem for Legendre polynomials. These equations are generally applicable for any angle of scatter, hence for $a$ and $b$ either positive or negative. In restricting the symbols $a, b$, etc., to denote positive magnitudes, we simply show $\bar{a}, \bar{b}$, etc., explicitly to identify a negative direction. Thus, e.g., the phase function for a ray scattered from a positive direction $\left(a, \phi_{0}\right)$ to a direction $\left(b, \phi_{1}\right)$ in the reflection side of the scatterer will be written.

$$
\mathrm{p}_{\mathrm{a} \overrightarrow{\mathrm{b}}}=\sum_{\ell=0}^{\mathrm{N}} \omega_{\ell} \sum_{\mathrm{m}=0}^{\ell} \frac{(\ell-\mathrm{m}) !}{(\ell+\mathrm{m}) !}\left(2-\delta_{0 \mathrm{~m}}\right) \cos \mathrm{m}\left(\phi_{1}-\phi_{0}\right) \mathrm{P}_{\ell}^{\mathrm{m}}(\mathrm{a}) \mathrm{p}_{\ell}^{\mathrm{m}}(\overline{\mathrm{b}})
$$

Note that

$$
\mathrm{p}_{\mathrm{a} \overline{\mathrm{b}}}=\mathrm{p}_{\overline{\mathrm{a}} \mathrm{b}} \quad ; \quad \mathrm{p}_{\mathrm{ab}}=\mathrm{p}_{\bar{a} \bar{b}}
$$

because of the symmetry properties of the $\mathrm{P}_{l}^{\mathrm{m}}(\mathrm{x})$.

The total emergent flux which has scattered j times will be donoted by $\Phi_{j}$, the arguments $h$ and $\alpha$ are not (usually) explicitly written.

\section{Idealized Single Scattered Intensity}

In this part we will introduce the technique of constructing the equations which we wish to discuss by using the concept of probabilities, so that in the succeedingly more complex cases which follow, the developments will be more easily comprehended.

In this part we treat the idealized situation in which one considers that a neutron incident on a plate of scattering material interacts with this material just once, at most, after which no further interaction of any sort is allowed. Then we consider:

(1) The incident flux, $\Phi_{0}=1$ is incident at an angle $\theta_{0}$ such that $\sec \theta_{0}=\alpha$. Sometimes $\Phi_{0}$ is set equal to unity and sometimes it is written out explicitly as $\Phi_{0}$, without consistency, in the following equations.

(2) The probability that an incident particle will penetrate to a depth $\kappa$ without having a previous interaction is just $e^{-\alpha \kappa}$.

(3) The probability that the particle will interact in the element $\mathrm{d} \kappa$ at $\kappa$ is $\alpha \mathrm{d} \kappa$. 
(4) Having interacted in $\mathrm{d} \kappa$, the probability that the particle will be found to be scattered at an angle $\Theta$ is $\sigma(\Theta) / \sigma_{t}$ measured as a probability per unit steradian at $\Theta$. In an element of solid angle $d \Omega$ at $\Theta$, there fore, the probability of finding the particle is

$$
\frac{\sigma(\Theta) \mathrm{d} \Omega}{\sigma_{\mathrm{t}}}=\frac{\mathrm{p}(\Theta) \mathrm{d} \Omega}{4 \pi}
$$

where $\mathrm{p}(\Theta)$ is the phase function which may be considered to be defined by this equation.

The details of notation, coordinates, and conventions have already been given in Part B of this section.

Now an element of the idealized scattered flux will be given by multiplying the probabilities in items (2), (3), and (4) just listed. This gives

$$
\Phi_{11}(\Theta) \mathrm{d} \Omega=\mathrm{e}^{-\alpha \kappa} \alpha \mathrm{d} \kappa \mathrm{p}(\Theta) \mathrm{d} \Omega / 4 \pi
$$

Integrating $k$ over the thickness of the plate, from zero to $h$, we obtain:

$$
\Phi_{11}=\left(1-\mathrm{e}^{-\alpha \mathrm{h}}\right) \mathrm{p}(\Theta) / 4 \pi
$$

This result is in agreement with the simpler development in Section II of this report. For, if the scatterer is irradiated over an area $A$, and the scattering of intensity $A \Phi_{11}$ per steradian falls on a detector subtending $\Delta \Omega$ at the scatterer, we can immediately write the flux on the detector as

$$
\mathrm{A} \Phi_{11} \Delta \Omega=\mathrm{A} \Delta \Omega\left(1-\mathrm{e}^{-\alpha h}\right) \sigma(\Theta) / \sigma_{t} .
$$

Since $h=N_{t} \sigma_{t}$ this is exactly the same expression derived in Section II by another method.

\section{Emergent Singly Scattered Intensity}

The flux scattered in $d \kappa$ at $\kappa$ as described in Part $C$, is actually exponentially attenuated by further interaction before it emerges from the plate. On the reflection side, the probability of the scattered particle emerging is e $-\overline{\beta \kappa}$ where $\beta=\left|\sec \theta_{1}\right|$ and $\theta_{1}$ is the altitude angle of the emergent direction with respect to the normal to the plate. On the transmission side of the plate, however, the probability is different. It is $\mathrm{e}^{-\beta(h-K)}$. 
On the reflection side, an element of eme rgent singly scattered flux will be given by multiplying the probabilities in items (2), (3), and (4) of Part C by $e^{-\beta \kappa}$. Thus

$$
\mathrm{d}_{1}^{(\mathrm{s})} \mathrm{d} \Omega=\mathrm{e}^{-\alpha \kappa} \alpha \mathrm{d} \kappa \mathrm{p}(\Theta)(\mathrm{d} \Omega / 4 \pi) \mathrm{e}^{-\beta \kappa}
$$

which when integrated over $\kappa$ from 0 to $\mathrm{h}$ gives

$$
\Phi_{1}^{(s)}(\mathrm{b})=\frac{\Phi_{0} \mathrm{p}(\Theta) \alpha}{4 \pi(\alpha+\beta)}\left(1-\mathrm{e}^{-\mathrm{h}(\alpha+\beta)}\right) \text {. }
$$

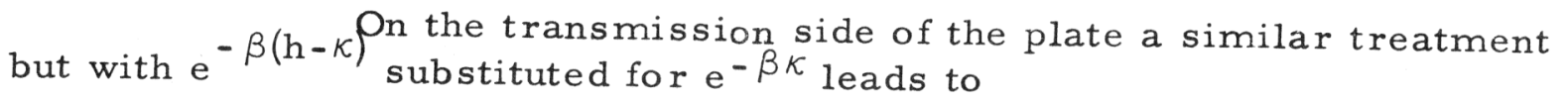

$$
\Phi_{1}^{(t)}(b)=\frac{\Phi_{0} p(\Theta) \alpha e^{-h \beta}}{4 \pi(\alpha-\beta)}\left(1-e^{-h(\alpha-\beta)}\right) \text {. }
$$

As in the previous Part $C$ of this section, the notation, sign, and coordinate conventions are those detailed in Part $B$.

Also in accordance with the conventions of Part $B$, we may replace $p(\Theta)$ by $p_{a b}$ in the transmission case and by $p_{a b}-$ in the re-
flection case to obtain

$$
\begin{aligned}
& \Phi_{1}^{(\mathrm{t})}(\mathrm{b})=\frac{\Phi_{0} \mathrm{p}_{\mathrm{ab}} \alpha \mathrm{e}^{-\mathrm{h} \alpha}}{4 \pi(\beta-\alpha)}\left(1-\mathrm{e}^{-\mathrm{h}(\beta-\alpha))}\right. \\
& \Phi_{1}^{(\mathrm{s})}(\mathrm{b})=\frac{\Phi_{0} \mathrm{p}_{\mathrm{ab}}-\alpha}{4 \pi(\beta+\alpha)}\left(1-\mathrm{e}^{-\mathrm{h}(\beta+\alpha))}\right.
\end{aligned}
$$

from which follow the useful relations

$$
\begin{aligned}
& \Phi_{1}^{(\mathrm{s})}(\alpha)=-\mathrm{e}^{-\mathrm{h} \alpha} \Phi_{1}^{(\mathrm{t})}(-\alpha) \\
& \Phi_{1}^{(\mathrm{t})}(\alpha)=-\mathrm{e}^{-\mathrm{h} \alpha} \Phi_{1}^{(\mathrm{s})}(-\alpha)
\end{aligned}
$$

because $\mathrm{p}_{\mathrm{ab}} \overline{\mathrm{b}}=\mathrm{p}_{\overline{\mathrm{ab}}}$, as noted in Part $\mathrm{B}$.

\section{E. Integrated Eme rgent Single Scattering}

The total single scattered flux which emerges is found by integrating the eme rgent flux over all outgoing directions on both sides of
the plate. We find

$$
\Phi_{1}=\Phi_{1}^{(t)}+\Phi_{1}^{(s)}
$$


where

$$
\begin{aligned}
& \Phi_{1}^{(t)}=\int_{0}^{2 \pi} \mathrm{d} \phi_{1} \int_{0}^{1} \mathrm{db} \Phi_{1}^{(t)}(\mathrm{b}) \\
& \Phi_{1}^{(s)}=\int_{0}^{2 \pi} \mathrm{d} \phi_{1} \int_{0}^{1} \mathrm{db} \Phi_{1}^{(s)}(\mathrm{b}) .
\end{aligned}
$$

The addition theorem for expansion of the phase function, Eq. (25), is used in equations (35) and (36) for $\cdot \Phi_{1}(\mathrm{t})$ ) and $\Phi_{1}(\mathrm{~s})$. The integration over $\phi_{1}$ is simple; it gives

$$
\int_{0}^{2 \pi} \mathrm{d} \phi_{1} \mathrm{p}(\mathrm{a}, \underline{\mathrm{b}})=2 \pi \sum_{l}^{\mathrm{N}} \stackrel{\omega}{l}_{l} \mathrm{P}_{l}(\mathrm{a}) \mathrm{P}_{l}(\stackrel{ \pm}{\mathrm{b}})
$$

where we have taken $\phi_{0}=0$.

We have then

$$
\begin{aligned}
& \Phi_{1}^{(t)}=\frac{\alpha e^{-h \alpha}}{2} \sum_{l \leqq 0}^{N} \omega_{l} P_{l}(a) \int_{0}^{1} d b P_{l}(b) \frac{1-e^{-h(\beta-\alpha)}}{\beta-\alpha} \\
& \Phi_{1}^{(s)}=\frac{\alpha}{2} \sum_{l=0}^{N} \omega_{l} P_{l} \text { (a) } \int_{0}^{1} \mathrm{db} P_{l}(\bar{b}) \frac{1-e^{-h(\beta+\alpha)}}{\beta+\alpha} .
\end{aligned}
$$

Now expand $\mathrm{P}_{l}(\mathrm{~b})$ in terms of the cosine so that

$$
P_{l}(\stackrel{t}{b})=\sum_{j=0}^{l \cdot} q_{l j}\left(\frac{b^{\prime}}{j}=\sum_{j=0}^{l} q_{l j}\left(\frac{ \pm}{\beta}\right)^{-j}\right.
$$

and we have

$$
\begin{aligned}
\int_{0}^{1} \mathrm{db} \mathrm{P}_{\ell}(\stackrel{ \pm}{\mathrm{b}}) & \frac{1-\mathrm{e}^{-h(\beta-\alpha)}}{\beta-\alpha}=\sum_{j=0}^{\ell} \mathrm{q}_{\ell j}( \pm)^{j} \int_{1}^{\infty} \frac{\mathrm{d} \beta\left(1-\mathrm{e}^{-h(\beta-\alpha)}\right)}{\beta^{j+2}(\beta-\alpha)} \\
& =\sum_{j=0}^{\ell}( \pm)^{j} \mathrm{q}_{\ell j} \mathrm{~F}_{j+2}(\mathrm{~h}, \alpha) .
\end{aligned}
$$

The functions $\mathrm{F}_{\mathrm{j}}(\mathrm{h}, \alpha)$ are discussed by Chandrasekhar* and others and are further discussed in Appendix $\mathrm{B}$ of this report. The coefficients $\mathrm{q}_{\ell j}$ are

*S. Chandrasekhar, Radiative Transfer, Clarendon Press, Oxford 1950, Appendix I. 
readily obtained from the equations for $\mathrm{P}_{l}(\mathrm{~b})$ as in Jahnke-Emde. ${ }^{*}$ They are given explicitly up to $l=j=4$ in the accompanying table.

TABLE OF q $\ell, j$

\begin{tabular}{|c|c|c|c|c|c|}
\hline$\ell$ & 0 & 1 & 2 & 3 & 4 \\
\hline 0 & 1 & & & & \\
\hline 1 & & 1 & & & \\
\hline 2 & $-1 / 2$ & & $3 / 2$ & & \\
\hline 3 & & $-3 / 2$ & & $5 / 2$ & \\
\hline 4 & $3 / 8$ & & $-30 / 8$ & & $35 / 8$ \\
\hline
\end{tabular}

In terms of the $F$ functions the solution for $\Phi_{1}$ is, then,

$$
\Phi_{1}=\frac{\alpha}{2} \sum_{l}^{N} \omega_{l} P_{l}(a) \sum_{j=0}^{\ell} q_{l j}\left\{e^{-h \alpha_{F}} F_{j+2}(h, \alpha)+(-)^{j} F_{j+2}(h,-\alpha)\right\} \text {. }
$$

In this equation the second $F$ function has a negative sign for the argument $\underline{\alpha}$ in accordance with the notation of Chandrasekhar, in view of the sign of the terms in $\underline{\alpha}$ in our Eq. 36 for $\Phi_{1}^{(s)}(\mathrm{b})$.

\section{F. Emergent Doubly Scattered Intensity}

The first scattering occurs in an element $\mathrm{d} \kappa$ at $\kappa$, the second in $\mathrm{d} \tau$ at $\tau$. On both the reflection side of the plate and the transmission side, the integrations must be broken up into two components, one for $\kappa>\tau$ and one for $\kappa<\tau$, so that four integrals in all are to be evaluated. The following tabulation itemizes limits of integration, probabilities of each differential process, etc., which are involved in constructing each of the four integrals in the same manner that was introduced in Part C above.

*E. Jahnke and F. Emde, Tables of Functions, G. E. Stechert and Co., New York. 


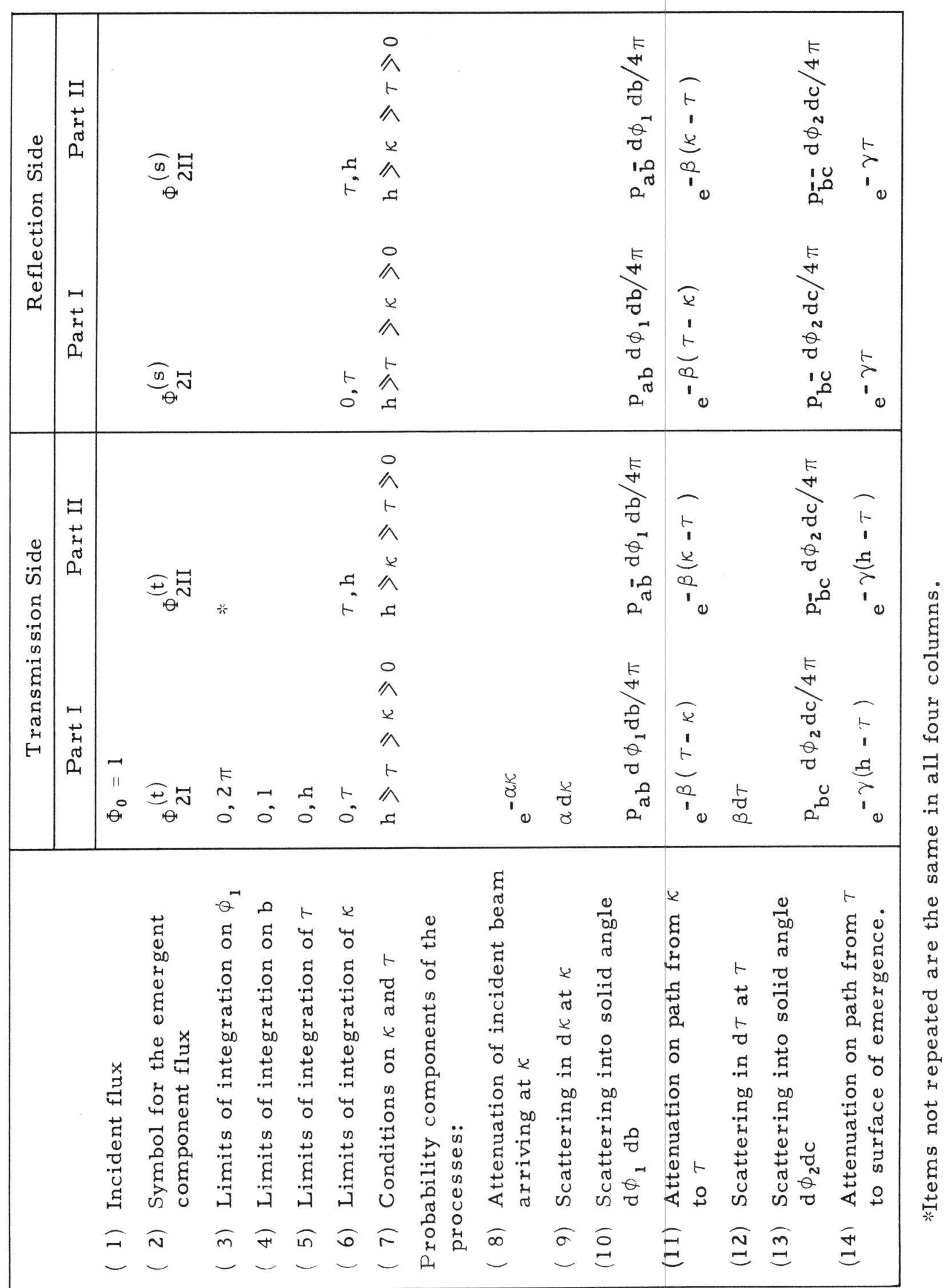


The four integrals are constructed by multiplying together the seven probability items, numbers (8) - (14) and applying the limits of integration, items (3) - (6).

The complete integral for $\Phi_{\text {II }}^{(t)}$ is, then

$\Phi_{2 \mathrm{I}}^{(\mathrm{t})} \mathrm{dcd} \phi_{2}=\frac{\Phi_{0} \alpha \mathrm{e}^{-\mathrm{h} \gamma}}{(4 \pi)^{2}} \mathrm{dcd} \phi_{2} \int_{0}^{2 \pi} \mathrm{d} \phi_{1} \int_{0}^{1} \mathrm{db} \beta \mathrm{p}_{\mathrm{ab}} \mathrm{p}_{\mathrm{bc}} \int_{0}^{\mathrm{h}} \mathrm{d} \tau \mathrm{e}^{-\tau(\beta-\gamma)} \int_{0}^{T} \mathrm{~d} \kappa \mathrm{e}^{\kappa(\beta-\alpha)}$ which may be integrated over $\kappa$ and $\tau$ to give

$\Phi_{2 I}^{(t)}(\mathrm{a}, \mathrm{c})=\frac{\Phi_{0} \alpha}{(4 \pi)^{2}} \int_{0}^{2 \pi} \mathrm{d} \phi_{1} \int_{0}^{l} \mathrm{db} \beta \mathrm{p}_{\mathrm{ab}} \mathrm{p}_{\mathrm{bc}}\left[\frac{1}{\beta-\alpha}\right]\left[\frac{\mathrm{e}^{-\mathrm{h} \alpha}-\mathrm{e}^{-\mathrm{h} \gamma}}{\gamma-\alpha}-\frac{\mathrm{e}^{-\mathrm{h} \beta}-\mathrm{e}^{-\mathrm{h} \gamma}}{\gamma-\beta}\right]$.

Similar solutions may be given for the remaining three components, $\Phi_{2 \mathrm{II}}^{(\mathrm{t})}, \Phi_{2 \mathrm{I}}(\mathrm{s})$, and $\Phi_{2 \mathrm{II}}(\mathrm{s})$. If one writes them out, simple substitution will validate the correctness of the following formalism:

$$
\begin{aligned}
& \Phi_{2 I I}^{(t)}(a, c)=\left(-e^{-h \alpha}\right)\left(e^{-h \gamma}\right) \Phi_{2 I}^{(t)}(-\alpha,-\gamma) \\
& \Phi_{2 I}^{(s)}(a, c)=\left(e^{-h \gamma}\right) \Phi_{2 I}^{(t)}(\alpha,-\gamma) \\
& \Phi_{2 I I}^{(s)}(a, c)=\left(-e^{-h \alpha}\right) \Phi_{2 I}^{(t)}(-\alpha, \gamma) .
\end{aligned}
$$

The only additional fact needed in corroborating these formal rules is the symmetry properties of the phase function as given in Eq. 27.

We now return to Eq. 47 and integrate first over $\phi_{1}$, next over $\mathrm{b}$. Expanding $\mathrm{P}_{\mathrm{ab}} \mathrm{P}_{\mathrm{bc}}$ by the expansion theorem for Legendre polynomials,

$$
\begin{aligned}
\mathrm{p}_{\mathrm{ab}} \mathrm{p}_{\mathrm{bc}}= & {\left[\sum_{\mathrm{k}}^{\mathrm{N}} \omega_{\mathrm{k}} \omega_{\mathrm{m}=0}^{\mathrm{k}}\left(2-\delta_{0 \mathrm{~m}}\right) \frac{(\mathrm{k}-\mathrm{m}) !}{(\mathrm{k}+\mathrm{m}) !} \mathrm{P}_{\mathrm{k}}^{\mathrm{m}}(\mathrm{a}) \mathrm{P}_{\mathrm{k}}^{\mathrm{m}}(\mathrm{b}) \cos \mathrm{m} \phi_{1}\right] } \\
& \times\left[\sum_{l=0}^{\mathrm{N}} \omega_{\ell} \sum_{\mathrm{n}=0}^{\ell}\left(2-\delta_{0 \mathrm{n}}\right) \frac{(l-\mathrm{n}) !}{(\ell+\mathrm{n}) !} \mathrm{P}_{l}^{\mathrm{n}}(\mathrm{b}) \mathrm{P}_{\ell}^{\mathrm{n}}(\mathrm{c}) \cos \mathrm{n}\left(\phi_{2}-\phi_{1}\right)\right],
\end{aligned}
$$

and making use of the fact that,

$$
\int_{0}^{2 \pi} \mathrm{d} \phi_{1} \cos \mathrm{m} \phi_{1} \cos \mathrm{n}\left(\phi_{2}-\phi_{1}\right)=\left\{\begin{array}{l}
0 \text { if } \mathrm{m} \neq \mathrm{n} \\
\left(2-\delta_{0 \mathrm{n}}\right)^{-1} 2 \pi \cos \mathrm{n} \phi_{2} \text { if } \mathrm{m}=\mathrm{n}
\end{array}\right.
$$


we obtain

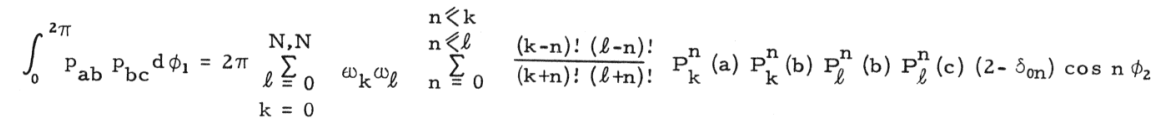

We now introduce the coefficients $g_{l, k ; j}^{n}$ in the expansion of the product of $\mathrm{P}_{k}^{\mathrm{n}}(\mathrm{b})$ and $\mathrm{P}_{\ell}^{\mathrm{n}}(\mathrm{b})$ by the defining equation:

$$
\frac{(k-n) !(l-n) !}{(k+n) !(l+n) !} P_{k}^{n}(b) P_{\ell}^{n}(b)=\sum_{j=0}^{\ell+k} b^{j} g_{l, k ; j}^{n}=\sum_{j=0}^{l+k} g_{l, k ; j}^{n} / \beta^{j} .
$$

The values of the $g_{l, k ; j}^{n}$ up to $l=k=4$ are given in a table appended. A useful check on any tabulation of these coefficients is the condition that

$$
\sum_{j=0}^{\ell+k} g_{\ell, k ; j}^{n}=\delta_{n, 0}
$$

By replacing db by its equivalent, $-d \beta / \beta^{2}$, we can now write, by aid of Eqs. 47, 52, and 53:

$$
\begin{aligned}
\Phi_{2 I}^{(t)}(a, c)= & \frac{\Phi_{0} \alpha}{8 \pi} \sum_{l=0}^{N, N} \omega_{k} \omega_{l} \sum_{n}\left(2-\delta_{0 n}\right) \cos n \phi_{2} P_{k}^{n}(a) P_{l}^{n}(c) \\
& \quad \sum_{j=0}^{\ell+k} g_{l, k ; j}^{n} e^{-h \gamma} \int_{0}^{h} d \tau e^{\tau \gamma} \int_{0}^{\tau} d \kappa e^{-\kappa \alpha} \frac{d \beta e^{-\beta(\tau-\kappa)}}{\beta^{j+l}} .
\end{aligned}
$$

The remaining integrals in this equation may now be considered solved in terms of the exponential integral functions $E_{n}(t)$, and repeated integration over these integral functions as defined by:

$$
\begin{aligned}
& E_{n}(t)=\int_{1}^{\infty} e^{-t u} d u / u^{n}, \\
& F_{n}(\tau, r)=\int_{0}^{T} e^{t r} E_{n}(t) d t, \\
& F_{n}(h, s, r)=\int_{0}^{h} e^{\tau s} F_{n}(\tau, r) d \tau
\end{aligned}
$$

These are further discussed in Appendix A and Appendix B. 
$\mathrm{g}_{\ell, \mathrm{k} ; \mathrm{j}}^{\mathrm{n}}$ for $\ell \leqslant 4$

\begin{tabular}{|c|c|c|c|c|c|c|c|c|c|c|c|}
\hline$\ell$ & $\mathrm{k}$ & $\mathrm{n}$ & $\mathbf{j}=0$ & $j=1$ & $j=2$ & $j=3$ & $j=4$ & $j=5$ & $j=6$ & $j=7$ & $j=8$ \\
\hline 0 & 0 & 0 & 1 & & & & & & & & \\
\hline 1 & 0 & 0 & 0 & 1 & & & & & & & \\
\hline 1 & 1 & 0 & 0 & 0 & 1 & & & & & & \\
\hline 1 & 1 & 1 & $1 / 4$ & 0 & $-1 / 4$ & & & & & & \\
\hline 2 & 0 & 0 & $-1 / 2$ & 0 & $3 / 2$ & & & & & & \\
\hline 2 & 1 & 0 & 0 & $-1 / 2$ & 0 & $3 / 2$ & & & & & \\
\hline 2 & 1 & 1 & 0 & $1 / 4$ & 0 & $-1 / 4$ & & & & & \\
\hline 2 & 2 & 0 & $1 / 4$ & 0 & $-3 / 2$ & 0 & $9 / 4$ & & & & \\
\hline 2 & 2 & 1 & 0 & 0 & $1 / 4$ & 0 & $-1 / 4$ & & & & \\
\hline 2 & 2 & 2 & $1 / 64$ & 0 & $-1 / 32$ & 0 & $1 / 64$ & & & & \\
\hline 3 & 0 & 0 & 0 & $-3 / 2$ & 0 & $5 / 2$ & & & & & \\
\hline 3 & 1 & 0 & 0 & 0 & $-3 / 2$ & 0 & $5 / 2$ & & & & \\
\hline 3 & 1 & 1 & $-1 / 16$ & 0 & $3 / 8$ & 0 & $-5 / 16$ & & & & \\
\hline 3 & 2 & 0 & 0 & $3 / 4$ & 0 & $-7 / 2$ & 0 & $15 / 4$ & & & \\
\hline 3 & 2 & 1 & 0 & $-1 / 16$ & 0 & $3 / 8$ & 0 & $-5 / 16$ & & & \\
\hline 3 & 2 & 2 & 0 & $1 / 64$ & 0 & $-1 / 32$ & 0 & $1 / 64$ & & & \\
\hline 3 & 3 & 0 & 0 & 0 & $9 / 4$ & 0 & $-15 / 2$ & 0 & $25 / 4$ & & \\
\hline 3 & 3 & 1 & $1 / 64$ & 0 & $-11 / 64$ & 0 & $35 / 64$ & 0 & $-25 / 64$ & & \\
\hline 3 & 3 & 2 & 0 & 0 & $1 / 64$ & 0 & $-1 / 32$ & 0 & $1 / 64$ & & \\
\hline 3 & 3 & 3 & $1 / 2304$ & 0 & $-1 / 768$ & 0 & $1 / 768$ & 0 & $-1 / 2304$ & & \\
\hline 4 & 0 & 0 & $3 / 8$ & 0 & $-15 / 4$ & 0 & $35 / 8$ & & & & \\
\hline 4 & 1 & 0 & 0 & $3 / 8$ & 0 & $-15 / 4$ & 0 & $35 / 8$ & & & \\
\hline 4 & 1 & 1 & 0 & $-3 / 16$ & 0 & $5 / 8$ & 0 & $-7 / 16$ & & & \\
\hline 4 & 2 & 0 & $-3 / 16$ & 0 & $39 / 16$ & 0 & $-125 / 16$ & 0 & $105 / 16$ & & \\
\hline 4 & 2 & 1 & 0 & 0 & $-3 / 16$ & 0 & $5 / 8$ & 0 & $-7 / 16$ & & \\
\hline 4 & 2 & 2 & $-1 / 384$ & 0 & $9 / 384$ & 0 & $-15 / 384$ & 0 & $7 / 384$ & & \\
\hline 4 & 3 & 0 & 0 & $-9 / 16$ & 0 & $105 / 16$ & 0 & $-255 / 16$ & 0 & $175 / 16$ & \\
\hline 4 & 3 & 1 & 0 & $3 / 64$ & 0 & $-25 / 64$ & 0 & $57 / 64$ & 0 & $-35 / 64$ & \\
\hline 4 & 3 & 2 & 0 & $-1 / 384$ & 0 & $9 / 384$ & 0 & $-15 / 384$ & 0 & $7 / 384$ & \\
\hline 4 & 3 & 3 & 0 & $1 / 2304$ & 0 & $-3 / 2304$ & 0 & $3 / 2304$ & 0 & $-1 / 2304$ & \\
\hline 4 & 4 & 0 & $9 / 64$ & 0 & $-180 / 64$ & 0 & $1110 / 64$ & 0 & $-2100 / 64$ & 0 & $1225 / 64$ \\
\hline 4 & 4 & 1 & 0 & 0 & $9 / 64$ & 0 & $-51 / 64$ & 0 & $91 / 64$ & 0 & $-49 / 64$ \\
\hline 4 & 4 & 2 & $1 / 2304$ & 0 & $-16 / 2304$ & 0 & $78 / 2304$ & 0 & $-112 / 2304$ & 0 & $49 / 2304$ \\
\hline 4 & 4 & 3 & 0 & 0 & $1 / 2304$ & 0 & $-3 / 2304$ & 0 & $3 / 2304$ & 0 & $-1 / 2304$ \\
\hline 4 & 4 & 4 & $1 / 147456$ & 0 & $-4 / 147456$ & 0 & $6 / 147456$ & 0 & $-4 / 147456$ & 0 & $1 / 147456$ \\
\hline
\end{tabular}


We also introduce the notation

$$
\mathrm{P}_{\mathrm{k} l}^{\mathrm{n}}(\mathrm{a}, \mathrm{c})=\left(1-\frac{1}{2} \delta_{\mathrm{k} l}\right)\left[\mathrm{P}_{\mathrm{k}}^{\mathrm{n}}(\mathrm{a}) \mathrm{P}_{\ell}^{\mathrm{n}}(\mathrm{c})+\mathrm{P}_{\ell}^{\mathrm{n}}(\mathrm{a}) \mathrm{P}_{\mathrm{k}}^{\mathrm{n}}(\mathrm{c})\right]
$$

to reduce the summation over all values of $k$ and $l$ to an ordered summation of less terms. Thus, finally, we have

$\Phi_{21}^{(t)}(a, c)=\frac{\Phi_{0} \alpha}{8 \pi} \sum_{l \geq k \geq 0}^{\ell=N} \omega_{\ell} \omega_{k} \sum_{n=0}^{k}\left(2-\delta_{0 n}\right) \cos n \phi_{2} P_{k \ell}^{n}(a, c) \sum_{j=0}^{l+k} g_{\ell, k ; j}^{n} e^{-h \gamma_{F}} f_{j+1}(h, \gamma-\alpha, \alpha)$

It is easily shown by an integration by parts that

$$
F_{n}(h, s, r)=e^{h s} F_{n}(h,-s, s+r) \text {. }
$$

It is also readily shown, using the symmetry properties of the Legendre polynomials, that

$$
\begin{aligned}
& \mathrm{P}_{\mathrm{k} l}^{\mathrm{n}}(\overline{\mathrm{a}}, \overline{\mathrm{c}})=(-)^{l+\mathrm{k}} \mathrm{P}_{\mathrm{k} l}^{\mathrm{n}} l^{(\mathrm{a}, \mathrm{c})} \\
& \mathrm{P}_{\mathrm{k} l}^{\mathrm{n}}(\mathrm{a}, \overline{\mathrm{c}})=(-)^{\ell+\mathrm{k}} \mathrm{P}_{\left.\mathrm{k} l^{\mathrm{n}}, \mathrm{c}\right)} .
\end{aligned}
$$

One final relation that aids in simplifying the final solution is that the $\mathrm{g}_{\ell, \mathrm{k}}^{\mathrm{n}} \mathrm{j}$ are zero if $(l+\mathrm{k})$ is odd unless $\mathrm{j}$ is also odd, and vice versa, when $(l+k)$ is even they are zero unless $j$ is even. Therefore the term $(-) l+k$ inside a sum over $l, k$, and $j$ may be replaced by $(-)^{j}$. Using this fact and the relations (49), (59), (60) and (61), we may now write the final answers

$$
\Phi_{2}^{(t)}(a, c)=\Phi_{2 I}^{(t)}+\Phi_{2 I I}^{(t)}=\Phi_{0} \sum_{l \geq k \geq 0}^{l=N} \omega_{l} \omega_{k} Q_{l k}^{(t)}\left(h, \alpha, \gamma, \phi_{2}\right)
$$

where

$$
\begin{aligned}
Q_{l, k}^{(t)}\left(h, \alpha, \gamma, \phi_{2}\right)= & \frac{\alpha}{8 \pi} \sum_{n=0}^{k}\left(2-\delta_{0 n}\right) \cos n \phi_{2} P_{k, \ell}^{n}(a, c) x \\
& \sum_{j=0}^{\ell+k} g_{\ell, k ; j}^{n} e^{-h \alpha}\left[F_{j+1}(h, \alpha-\gamma, \gamma)+(-)^{j}+F_{j+1}(h, \alpha-\gamma,-\alpha)\right]
\end{aligned}
$$

and

$$
\Phi_{2}^{(\mathrm{s})}(\mathrm{a}, \mathrm{c})=\Phi_{2 \mathrm{I}}^{(\mathrm{s})}+\Phi_{2 \mathrm{II}}^{(\mathrm{s})}=\Phi_{0} \sum_{l \geq \mathrm{k} \geq 0}^{\ell=N} \omega_{l} \omega_{\mathrm{k}} \mathrm{Q}_{l \mathrm{k}}^{(\mathrm{s})}\left(\mathrm{h}, \alpha, \gamma, \phi_{2}\right)
$$


where

$$
\begin{aligned}
\mathrm{Q}_{\ell k}^{(s)}\left(h, \alpha, \gamma, \phi_{2}\right)= & \frac{\alpha}{8 \pi} \sum_{n=0}^{k}\left(2-\delta_{0 n}\right) \cos n \phi_{2} P_{k \ell}^{n}(a, \bar{c}) x \\
& \sum_{j=0}^{l+k} g_{\ell, k ; j}^{n}\left[F_{j+1}(h,-\alpha-\gamma, \alpha)+(-)^{j} F_{j+1}(h,-\alpha-\gamma, \gamma,)\right] .
\end{aligned}
$$

G. The Integrated Doubly Scattered Flux

Next consider the total emergent doubly scattered flux

$$
\Phi_{2}=\int_{0}^{2 \pi} \mathrm{d} \phi_{2}\left\{\int_{0}^{1} \mathrm{dc} \Phi_{2}^{(\mathrm{t})}\left(\mathrm{c}, \phi_{2}\right)+\int_{-1}^{0} \mathrm{dc} \Phi_{2}^{(s)}\left(\mathrm{c}, \phi_{2}\right)\right\} .
$$

A c rude approximation for this integral can be obtained in the following manner: Vineyard* has shown that the ratio $\Phi_{n} / \Phi_{n-1}$ is approximately independent of the order, $n$, of scattering, at least for the "quasi-isotropic" case which he considers. This assumption permits the evaluation of the sum of emergent scattered flux for all orders of scatter as

$$
\sum_{n=1}^{\infty} \Phi_{n}=\Phi_{1}^{2} /\left(\Phi_{1}-\Phi_{2}\right)
$$

Since, in the cases considered here, this sum must also converge to $1-e^{-\alpha h}$, we have

$$
\Phi_{2} \simeq \Phi_{1}\left[1-\frac{\Phi_{1}}{1-\mathrm{e}^{-\alpha h}}\right] .
$$

The following exact evaluation of this integral is somewhat tedious. The integration over the azimuthal angle is trivial giving

$\Phi_{2}=\frac{\alpha}{4} \sum_{k}^{N} \sum_{l=k}^{N} \omega_{\ell} \omega_{k} \sum_{j=0}^{2 N} g_{\ell, k ; j}^{0} \frac{2-\delta_{\ell k}}{2}$

$$
\left\{\begin{aligned}
P_{l}(a) & \sum_{s=0}^{k} q_{k s}\left(I_{s, j+1}+J_{s, j+1}\right) \\
+P_{k}(a) & \sum_{s=0}^{k} q_{\ell s}\left(I_{s, j+1}+J_{s, j+1}\right)
\end{aligned}\right\}
$$

where we have made use of the definition (Eq. 58) of $\mathrm{P}_{l_{k}}^{0}(\mathrm{a}, \mathrm{c})$ and have expanded $\mathrm{P}_{\mathrm{k}}(\mathrm{c})$ in powers of $\mathrm{c}$ as before. The integrals $\mathrm{I}$ and $\mathrm{J}$ are defined by

*George H. Vineyard, Phys. Rev. 96, 93 (1954) 


$$
I_{s, j+1}(h, \alpha)=I_{s, j+1}^{(1)}(h, \alpha)+(-)^{j} I_{s, j+1}^{(2)}(h, \alpha)
$$

and

$$
J_{s, j+1}(h, \alpha)=(-)^{s+1}\left\{J_{s, j+1}^{(1)}(h, \alpha)+(-)^{j} J_{s, j+1}^{(2)}(h, \alpha)\right\},
$$

in terms of the definite integrals

$$
\begin{aligned}
& I_{s, j+1}^{(1)}(h, \alpha)=\int_{0}^{h} d \tau \int_{1}^{\infty} \frac{d \gamma}{\gamma^{s+2}} e^{(\gamma-\alpha) \tau} e^{-\gamma h} F_{j+1}(\tau, \alpha), \\
& I_{s, j+1}^{(2)}(h, \alpha)=\int_{0}^{h} d \tau \int_{1}^{\infty} \frac{d \gamma}{\gamma^{s+2}} e^{(\gamma-\alpha) \tau} e^{-\gamma h} F_{j+1}(h-\tau,-\alpha), \\
& J_{s, j+1}^{(1)}(h, \alpha)=\int_{0}^{h} d \tau \int_{1}^{\infty} \frac{d \gamma}{\gamma^{s+2}} e^{-(\gamma+\alpha) \tau} F_{j+1}(\tau, \alpha) \\
& J_{s, j+1}^{(2)}(h, \alpha)=\int_{0}^{h} d \tau \int_{1}^{\infty} \frac{d \gamma}{\gamma^{s+2}} e^{-(\gamma+\alpha) \tau} F_{j+1}(h-\tau,-\alpha) .
\end{aligned}
$$

A simple change of variables shows that

$$
J_{s, j+1}^{(1)}(h, \alpha)=e^{-\alpha h} I_{s, j+1}^{(2)}(h,-\alpha)
$$

and

$$
J_{s, j+1}^{(2)}(h, \alpha)=e^{-\alpha h} I_{s, j+1}^{(1)}(h,-\alpha)
$$

Thus

$$
\Phi_{2}=\sum_{k=0}^{N} \sum_{l=k}^{N} \omega_{l} \omega_{k} \Lambda_{l k} \quad
$$

where

$$
\begin{aligned}
\Lambda_{\ell k}(h, \alpha)= & \frac{\alpha}{4} \frac{2-\delta_{\ell k}}{2} \sum_{j=0}^{2 N} \sum_{s}^{N} g_{l, k ; j}^{0}\left[q_{k s} P_{l}(a)+q_{\ell, s} P_{k}(a)\right] \\
& \times\left\{I_{s, j+1}(h, \alpha)+(-)^{s+j+1} e^{-\alpha h} I_{s, j+1}(h,-\alpha)\right\} .
\end{aligned}
$$

The evaluation of the integral $I_{s, j+1}$ is discussed in Appendices $C$ and $D$. 
SECTION IV.

\section{PLANS FOR FUTURE ANGULAR DISTRIBUTION DATA}

It will soon be possible to obtain scattering data, using this equipment, with the detectors mounted on a circular track, at angles other than the five fixed angles at which these results were obtained. These additional data will permit a somewhat more critical analysis than has been possible previously. It is believed that in this way the major uncertainties remaining in the present results can be eliminated.

Briefly the planned modifications of the present analysis are: 1) Correction for the finite angular resolution of the detecting apparatus. 2) A least square analysis for the coefficients in a series of Legendre polynomials containing seven terms (more if warranted) for the differential cross section, and 3) An internally coded iteration process for the multiple scattering corrections.

The quantity actually measured in these experiments is the cross section averaged not only over the energy spectrum of the incident beam but also over the solid angle of the detector, i.e.,

$$
\sigma_{M}\left(\mu_{j}\right)=\int_{\Delta \Omega_{j}} d \Omega_{j} \epsilon_{j}(\mu) \lambda_{j}(\mu) \sigma(\mu) / \int_{\Delta \Omega_{j}} d \Omega_{j} \epsilon_{j}(\mu) \lambda \lambda_{j}(\mu),
$$

where $\sigma_{M}\left(\mu_{j}\right)$ is the measured cross section evaluated at the mean scattering angle of the $\mathrm{j}^{\text {th }}$ counter; $\epsilon_{j}(\mu)$ is the efficiency of this counter, and $\lambda_{j}(\mu)$ is a function describing the geometry of the experiment. Thus Eq. (12) should be written

$$
\sigma_{M}\left(\mu_{j}\right)=\sum_{\ell} a_{\ell}\left\{\int_{\Delta \Omega_{j}} d \Omega_{j} \epsilon_{j}(\mu) \lambda_{j}(\mu) P_{\ell}(\mu) / \int_{\Delta \Omega j} d \Omega_{j} \epsilon_{j}(\mu) \lambda_{j}(\mu)\right\}
$$

assuming that $\epsilon_{\mathrm{j}}$ is not a function of $\mu(i . e$. , that the counter system is equally efficient over its entire face) and using the $\lambda_{j}(\mu)$ appropriate to this geometry, we have evaluated these integrals numerically for the five scattering angles at which measurements were made. These corrections were made independently for that portion of the data for which the plura] scattering corrections were made. The results are shown in the cross section graphs.

A least square analysis carried out for the differential cross section of $\mathrm{Pd}$ at $578 \mathrm{kev}$ gives

$$
\sigma(\mu)=.558 \pm .013+(.542 \pm .019) \mathrm{P}_{1}(\mu)+(.324 \pm .020) \mathrm{P}_{2}(\mu)
$$


A comparison of the experimental (statistical) uncertainties with the least square errors is given in tabular form below:

\begin{tabular}{|c|c|c|}
\hline$\mu$ & $\sigma(\mu)$ - measured & $\sigma(\mu)$ - least square \\
\hline .92 & $1.35 \pm .02$ & $1.33 \pm .04$ \\
\hline .58 & $0.86 \pm .02$ & $0.87 \pm .03$ \\
\hline-.03 & $0.38 \pm .01$ & $0.37 \pm .02$ \\
\hline-.38 & $0.27 \pm .01$ & $0.25 \pm .02$ \\
\hline-.81 & $0.27 \pm .01$ & $0.28 \pm .03$ \\
\hline
\end{tabular}

This agreement is a measure of the adequacy of a three-term expansion of this differential cross section.

The iteration process which we have used to evaluate the plural scattering corrections would converge more rapidly (at most three iterations for $\left.h=N \sigma_{t} \simeq 0.5\right)$ if the total cross section could be obtained independently. Because of this some consideration is being given to the problem of obtaining transmission data simultaneously with the angular distribution data. Also this would make unnecessary the normalization of all total cross sections to that of a given sample of graphite.

The mobility of the counters will permit also a direct intercalibration of the efficiency of the counters for a given neutron energy. In order to obtain the energy dependence of the counter efficiency more accurately we intend to make use of the known angular distribution and energy degradation effect of a hydrogen scatterer.

Several measurements of the angular distribution of neutrons scattered from a single resonance have been carried out with good resolution. Unfortunately the correction techniques which are described in this report are no longer applicable for this type of data because of the rapid variation with energy of the measured cross section. We are presently considering a Monte Carlo calculation which will include possibly the geometrical as well as the multiple scattering corrections for the se data.This should provide also a worthwhile check on the "poor resolution" analysis as used in this report.

Finally we believe that the flexibility of this equipment, together with its high counting efficiencies, will permit both single and double scattering polarization experiments. 


\section{SECTION V}

\section{ACKNOWLEDGEMENTS}

We wish to thank Merle Burgy for his part in work with one of us (A. Langsdorf) which led to development of the type of equipment used in the present work.

Acknowledgement is also made for the vital aid of the staff of the van de Graaff machine, including particularly Jack $R$. Wallace, William F. Evans, Ronald L. Amrein, and Vincent A.Montelpasse. They helped in the setting up of the equipment, both mechanical and electronic, in preparing scattering samples and in performing the actual experiments.

Thanks are due to Donald A. Flanders, Herbert L. Gray, and others of the mathematics section for cooperation in machine computation of results of these experiments.

Thanks are due to George M. Lobell, Edward J. Lach and others of the machine shop staff for their part in construction of the equipment.

Thanks are due to Thomas Brill and the staff of the Electronics group for constructing most of the electronic equipment.

We are indebted to Professor S. Chandrasekhar for discussing with us the nature of the scattering corrections and the possible application of his general theory of scattering to our problem. 
SECTION VI

$\underline{\text { THE EXPERIMENTAL DATA }}$

Table 1 is an example of typical data. The large graphs which follow on pp. 57-137 give the complete results of the survey. The titles give detailed information for each graph.

Table 1

TYPICAL DATA

Date $2-16-55$, En $=477 \mathrm{kev}$

\begin{tabular}{|c|c|c|c|c|c|c|c|c|}
\hline \multirow[b]{2}{*}{ Run No. } & \multirow[b]{2}{*}{ E1 ement } & \multirow[b]{2}{*}{$\mathrm{N}_{\mathrm{t}}$} & \multirow[b]{2}{*}{ Monitor Counts } & \multicolumn{5}{|c|}{ Counts at } \\
\hline & & & & $22^{\circ}$ & $54^{\circ}$ & $92^{\circ}$ & $112^{\circ}$ & $144^{\circ}$ \\
\hline 254 & C & .05297 & 27762 & 20319 & 17653 & 14070 & 13304 & 12448 \\
\hline 255 & C & .02575 & 56172 & 22326 & 18372 & 14765 & 13364 & 12761 \\
\hline 256 & No sa & $\mathrm{np} 1 \mathrm{e}$ & 56045 & 3473 & 1413 & 753 & 846 & 1425 \\
\hline 275 & $\mathrm{Fe}$ & .02387 & 28348 & 13812 & 9691 & 6753 & 6197 & 6676 \\
\hline 480 & $\mathrm{Ra}-\mathrm{E}$ & esource & for 5 minutes & 3757 & 3814 & 3856 & 3777 & 3872 \\
\hline
\end{tabular}

Some general information on the graphs and their figure titles is as follows:

The "Series" number identifies the three groups of experiments carried through at three different times. Series 1 data was obtained in October 1954, Series 2 in February 1955, and Series 3 in August 1955.

The $\mathrm{N}_{\mathrm{t}}$ value is the thickness of a scattering sample measured in (atoms per $\mathrm{cm}^{2}$ ) $\times 10^{-24}$. The thickness in mean free path units is, then, $h=N_{t} \sigma_{t}$, where we consider that the total scattering cross section, $\sigma_{s t}$ equals the total cross section $\sigma_{t}$ to a sufficient accuracy for our purposes. The curves for total cross section, marked $\sigma_{t}$ on the graphs, should be considered as representing more closely the total scattering cross section than the total cross section; we should probably have used some other symbol than $\sigma_{t}$ on the graphs to make this clear.

The neutron energies at which the data points are shown are average energies. At each voltage setting of the van de Graaff machine, the maximum neutron energy was calculated from the voltage setting as standardized by the $\mathrm{Li}^{7}(\mathrm{p}, \mathrm{n})$ reaction threshold voltage of $1.882 \mathrm{Mev}$. Using the lithium layer thickness as determined by the rise curve above threshold, the energy loss of the protons in the lithium was computed using data on 
stopping power such as that given by Allison and Warshaw.* Then the minimum neutron energy was calculated and the average of this and the maximum was taken. There is considerable uncertainty as to whether the points plotted at $30 \mathrm{kev}$ (from Series 2 data only) and at $60 \mathrm{kev}$ in particular should properly be plotted at these positions. Better energy resolution must be used to resolve this question.

Figures 10 through 13 are photographs of cut-outs of the actual graphs, racked in order of increasing atomic number for each $\omega_{\bar{l}}$. A photograph from both the right and left end of each assembly is shown. In the former cases, the actual graph paper rulings show; in the latter, the plain cardboard backings were edged in black to emphasize the structure. We apologize for the crudeness of these figures, but they seem interesting enough to include nevertheless, since preparation of better figures would have delayed publication. For further general information see also notes in Figure titles on pp. 60, 62, etc., and comments on p. 50 (paragraph 3) and p. 9 (paragraph 4).

*Allison and Warshaw, Rev. Mod. Phys, 25, 779 (1953). 
GRAPHS AND FIGURES 


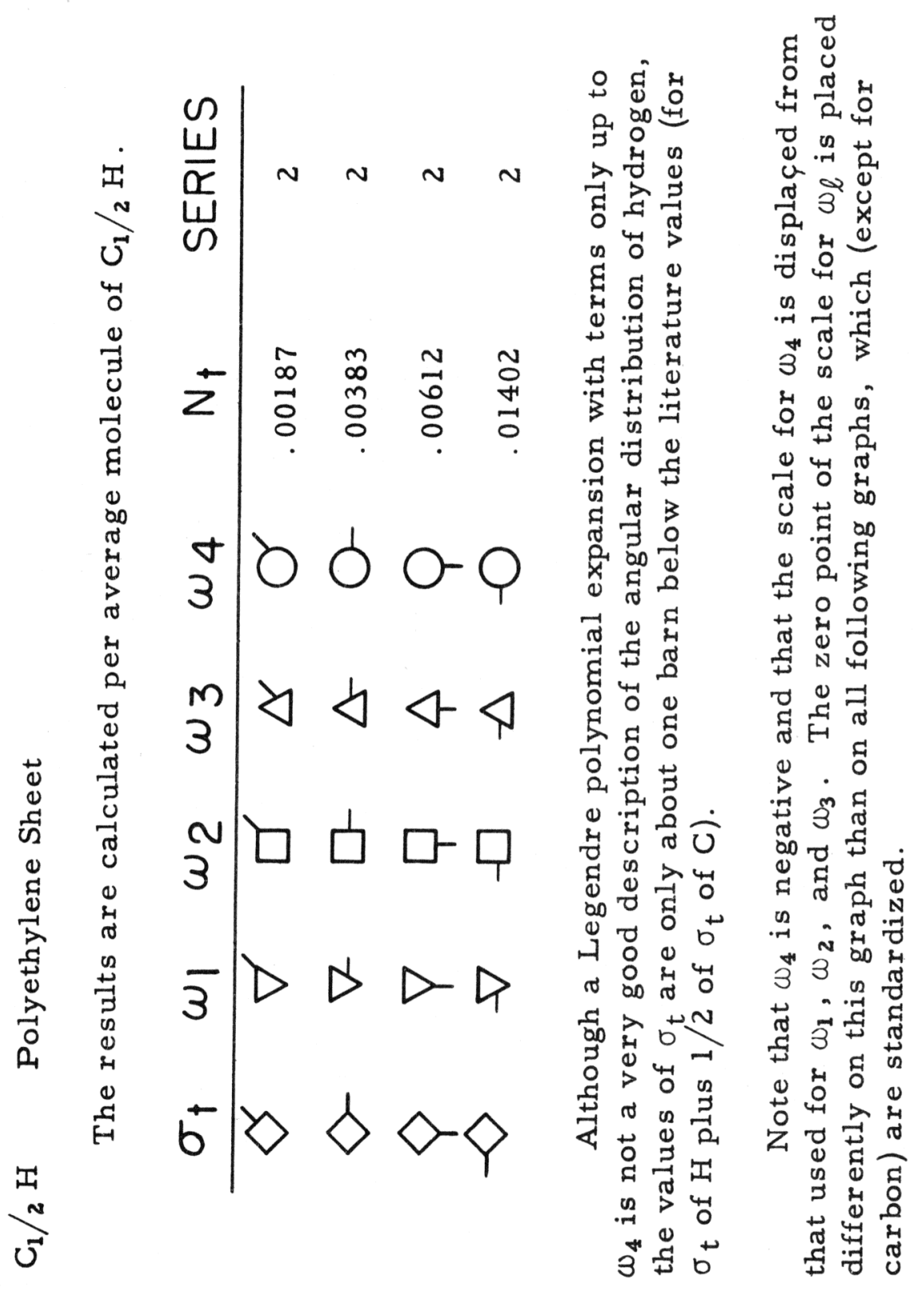



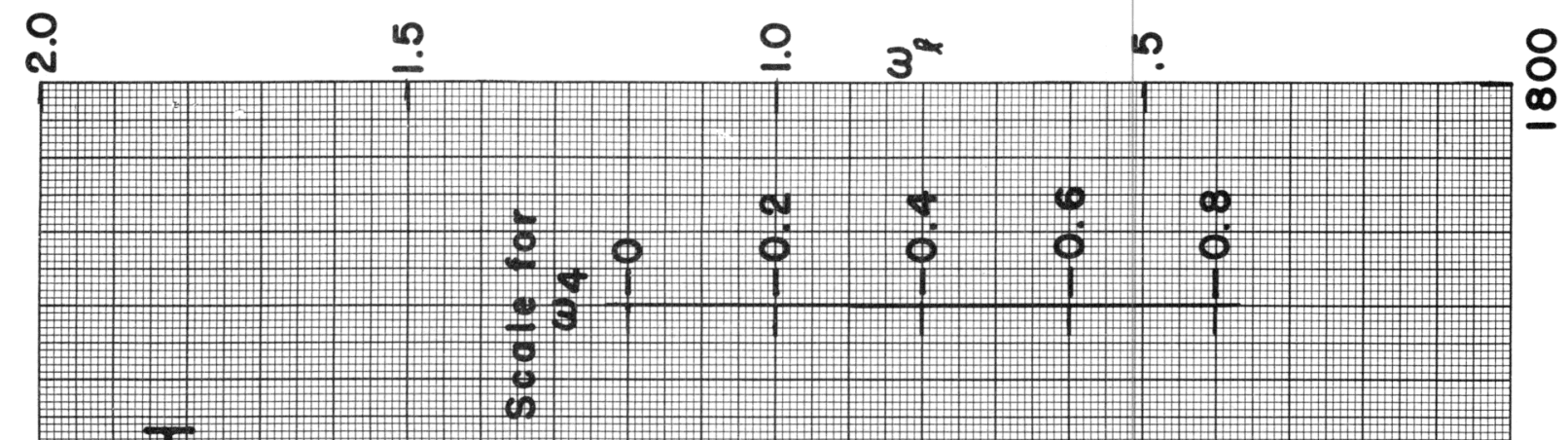

I
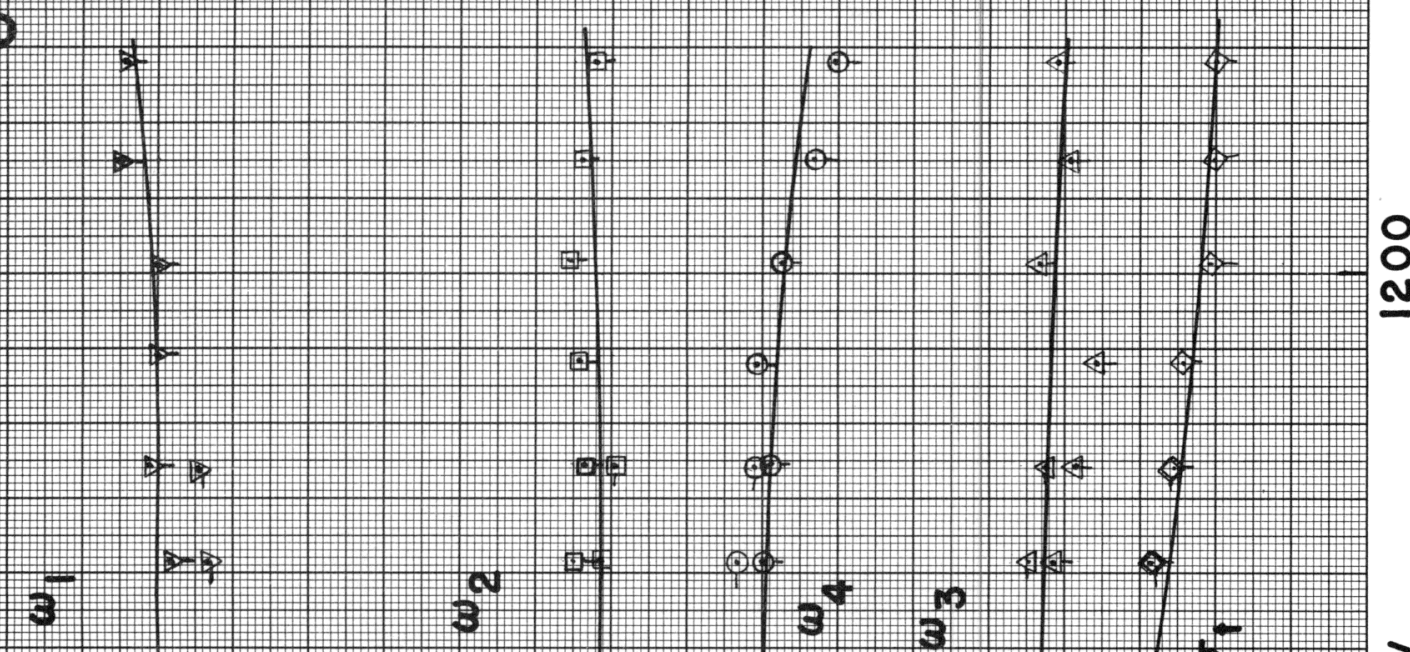

1 1
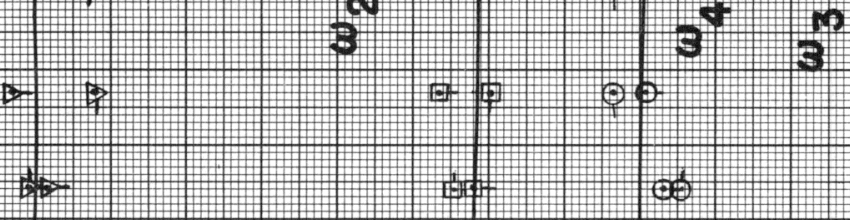

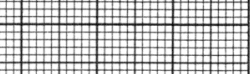


${ }_{4} \mathrm{Be} \quad$ Beryllium Metal Plates

\begin{tabular}{ccccccc}
$\sigma_{+}$ & $\omega_{1}$ & $\omega_{2}$ & $\omega_{3}$ & $\omega_{4}$ & $N_{+}$ & SERIES \\
\hline & $\nabla$ & $\square$ & $\Delta$ & $\bigcirc$ & .03277 & 1 \\
- & $\nabla$ & $\square$ & $\Delta$ & $O$ & .01857 & 2 \\
0 & $\nabla$ & $-\square$ & $\Delta$ & - & .03872 & 2
\end{tabular}

The data points for $\omega_{3}$ and $\omega_{4}$ are displaced two (small) divisions to the right and left, respectively, from the correct $E_{n}$ value, to decrease the overlap of symbols. 


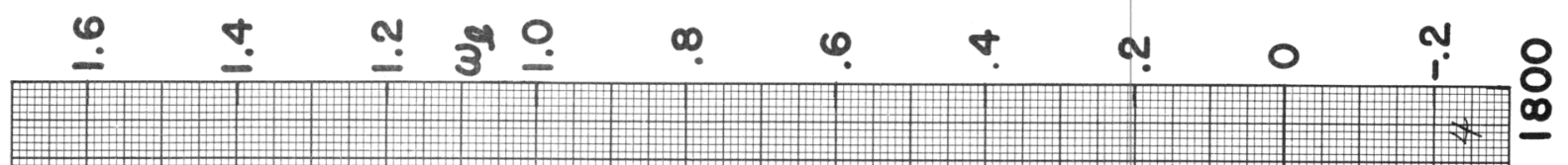

\section{at:}

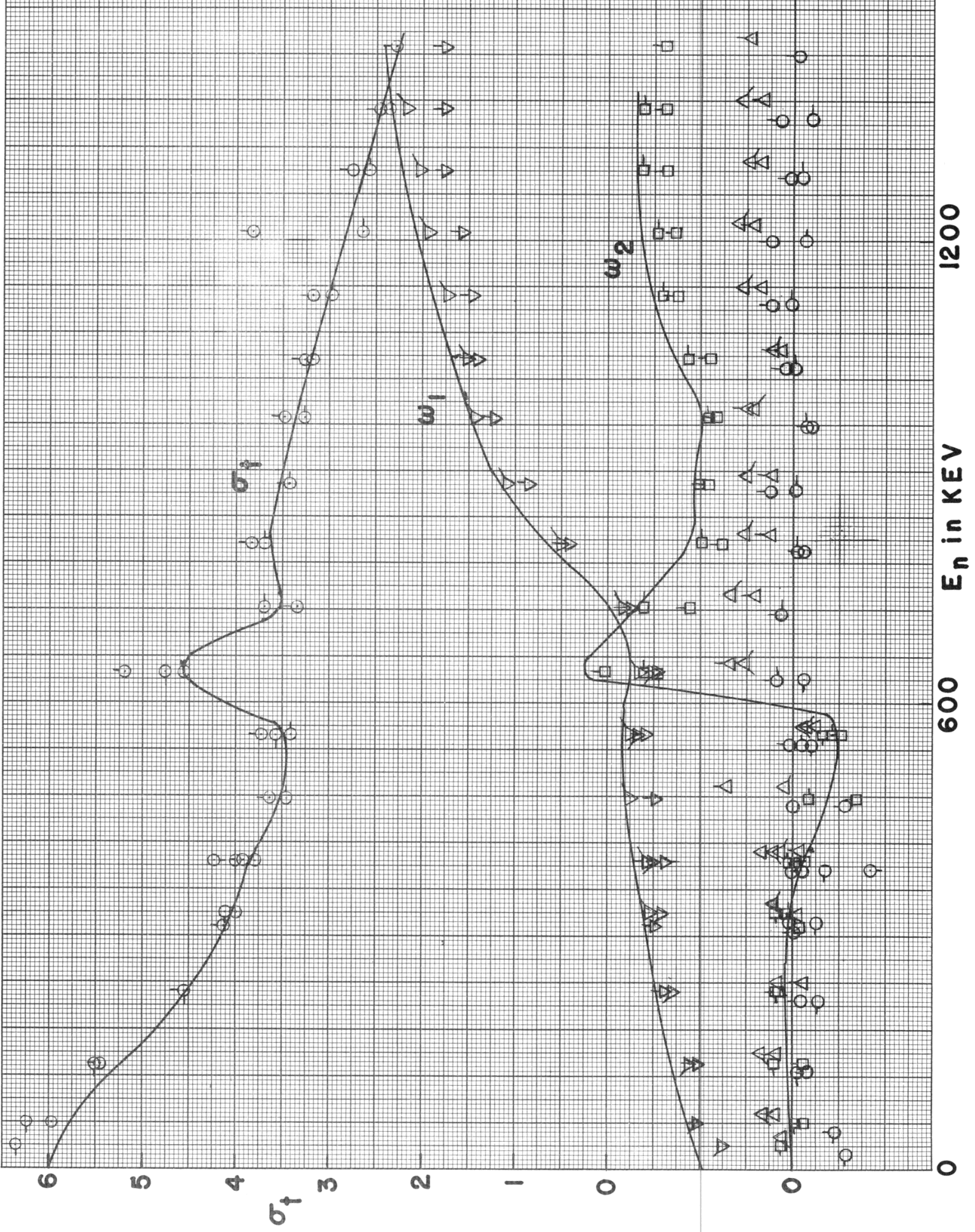




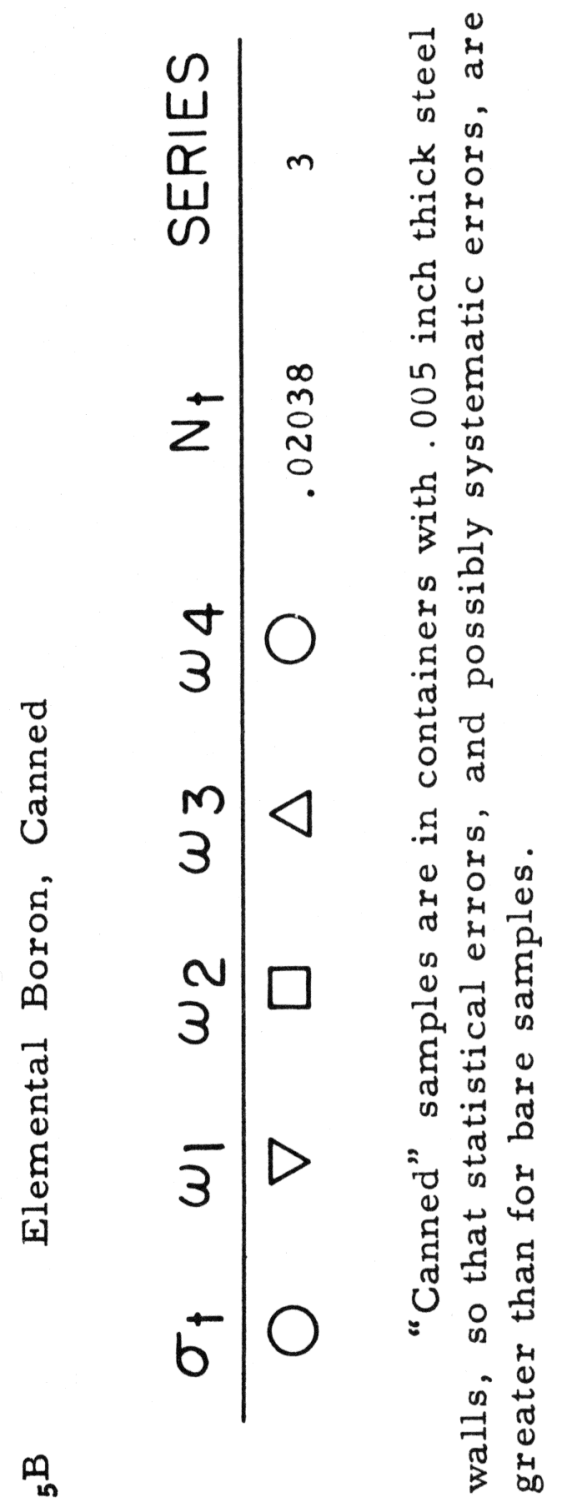




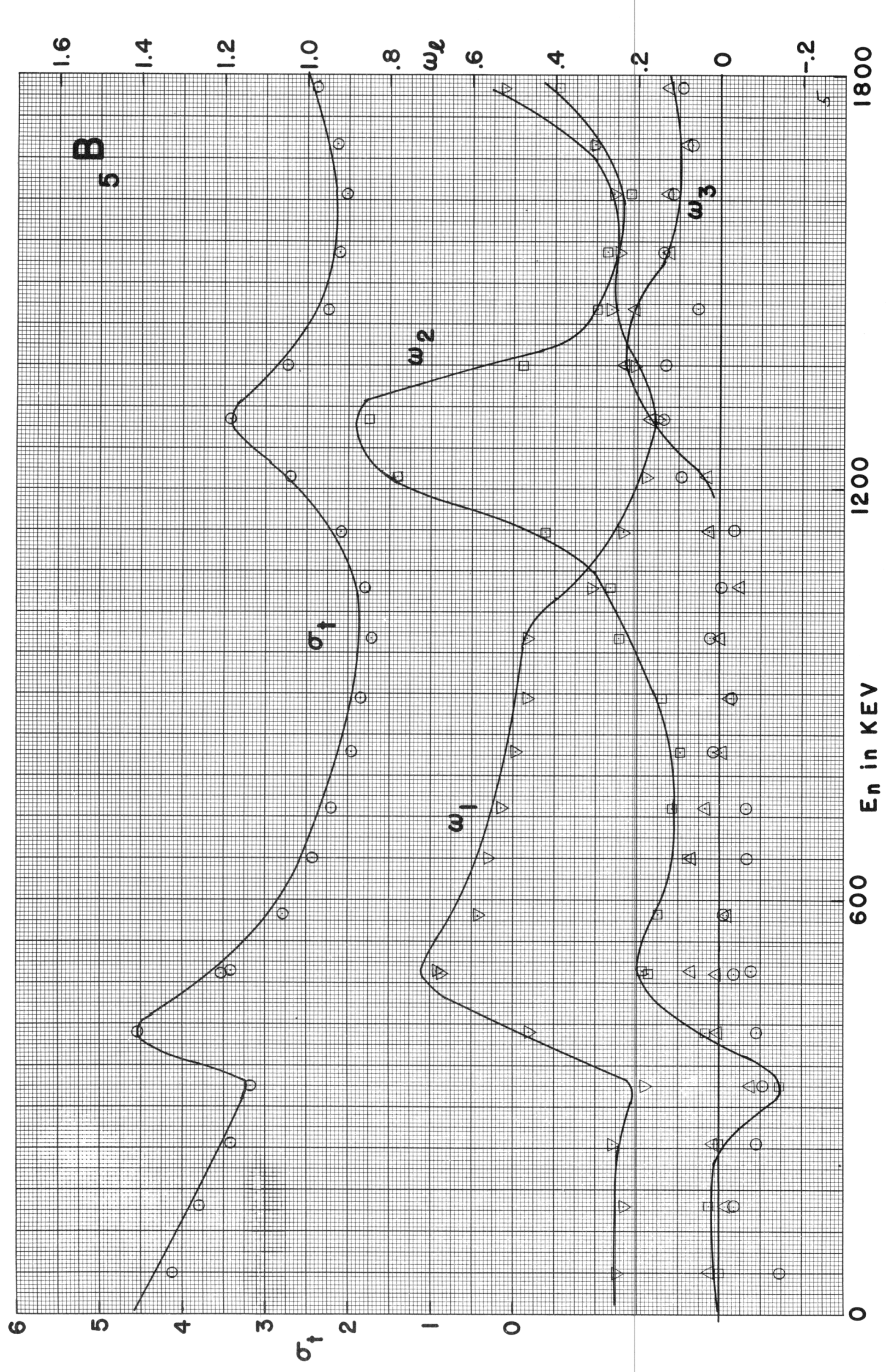




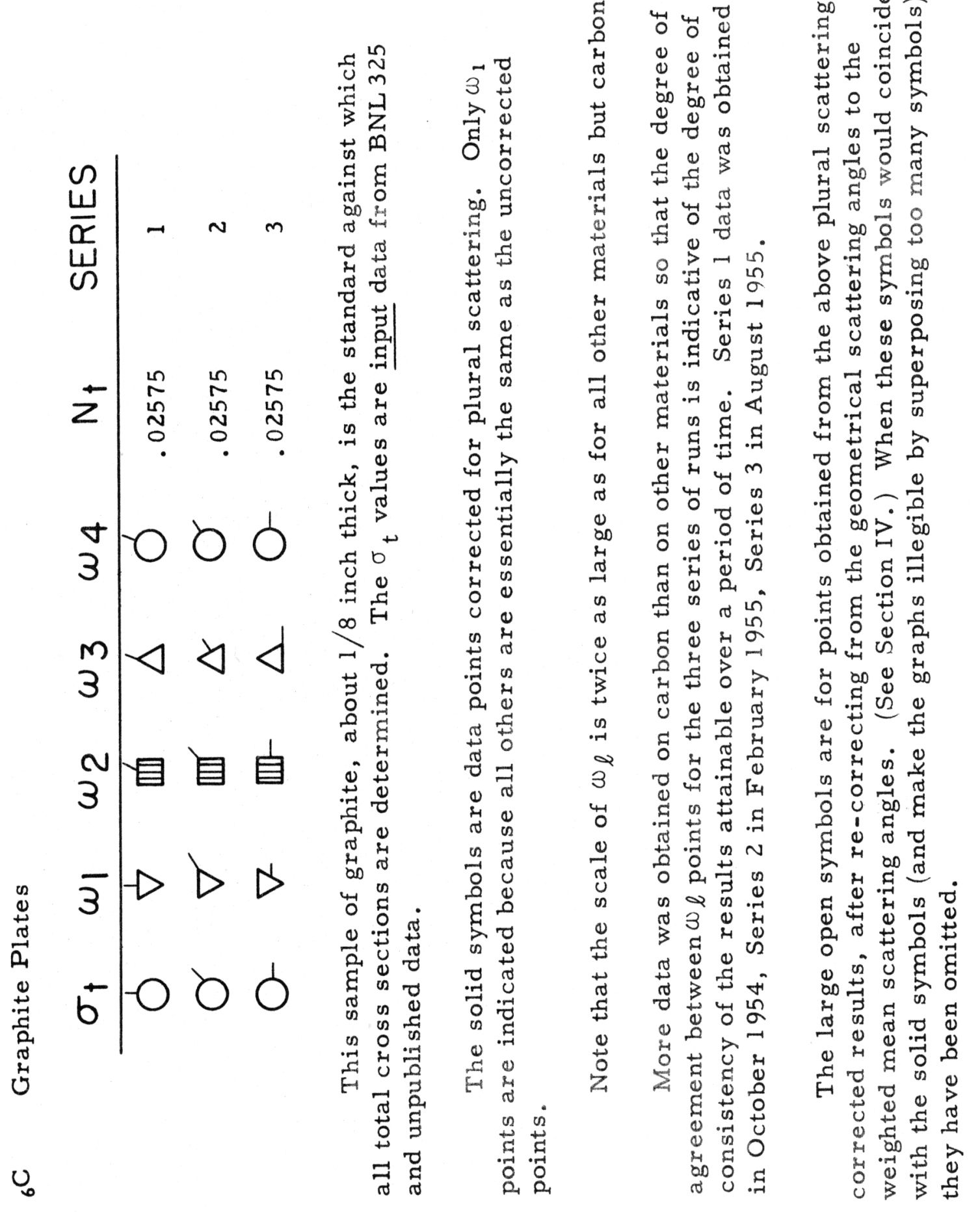




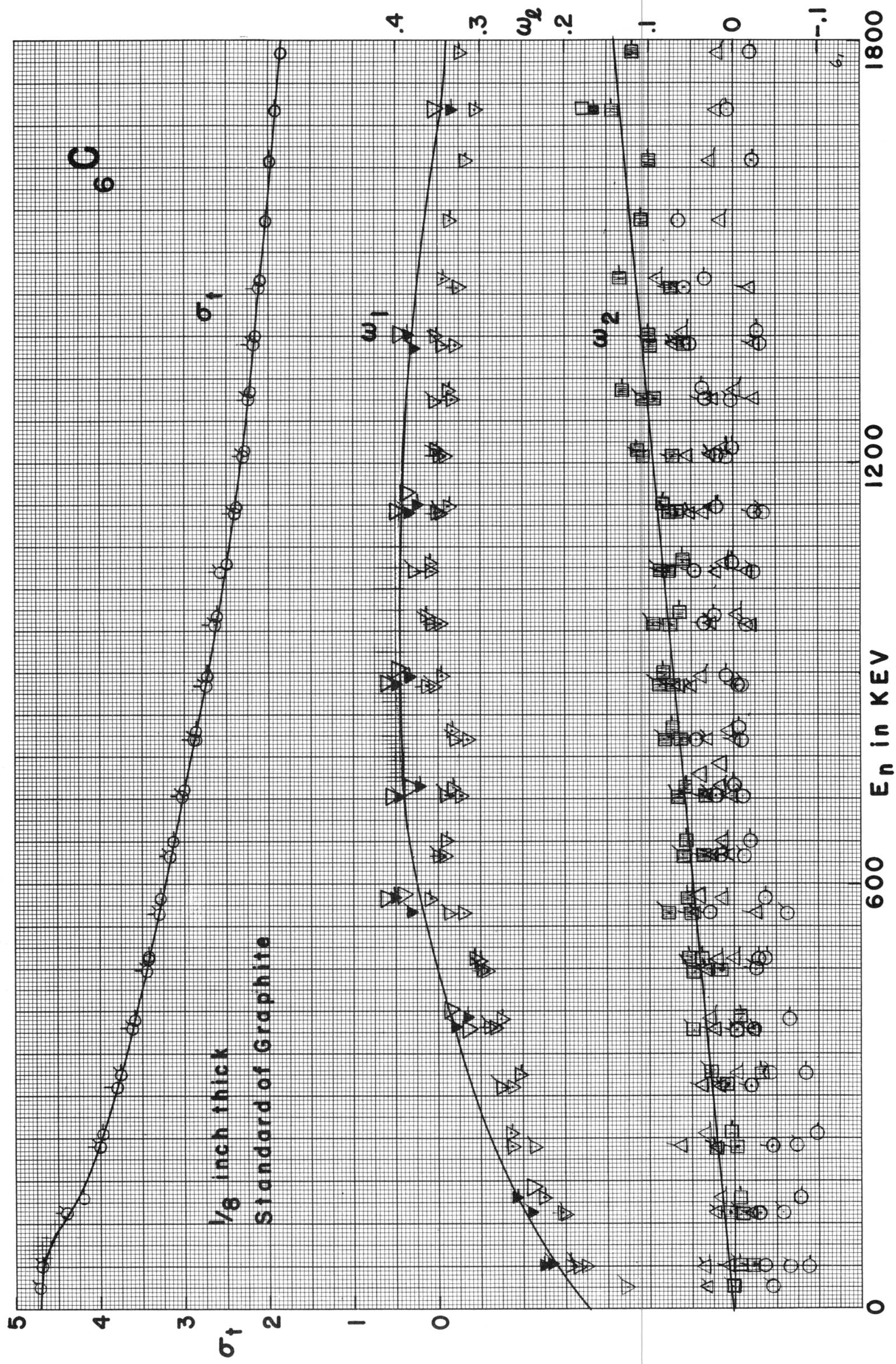




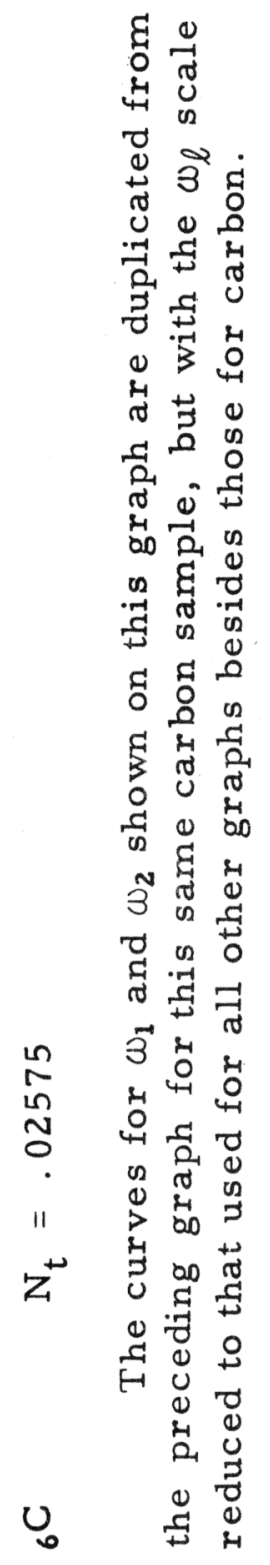




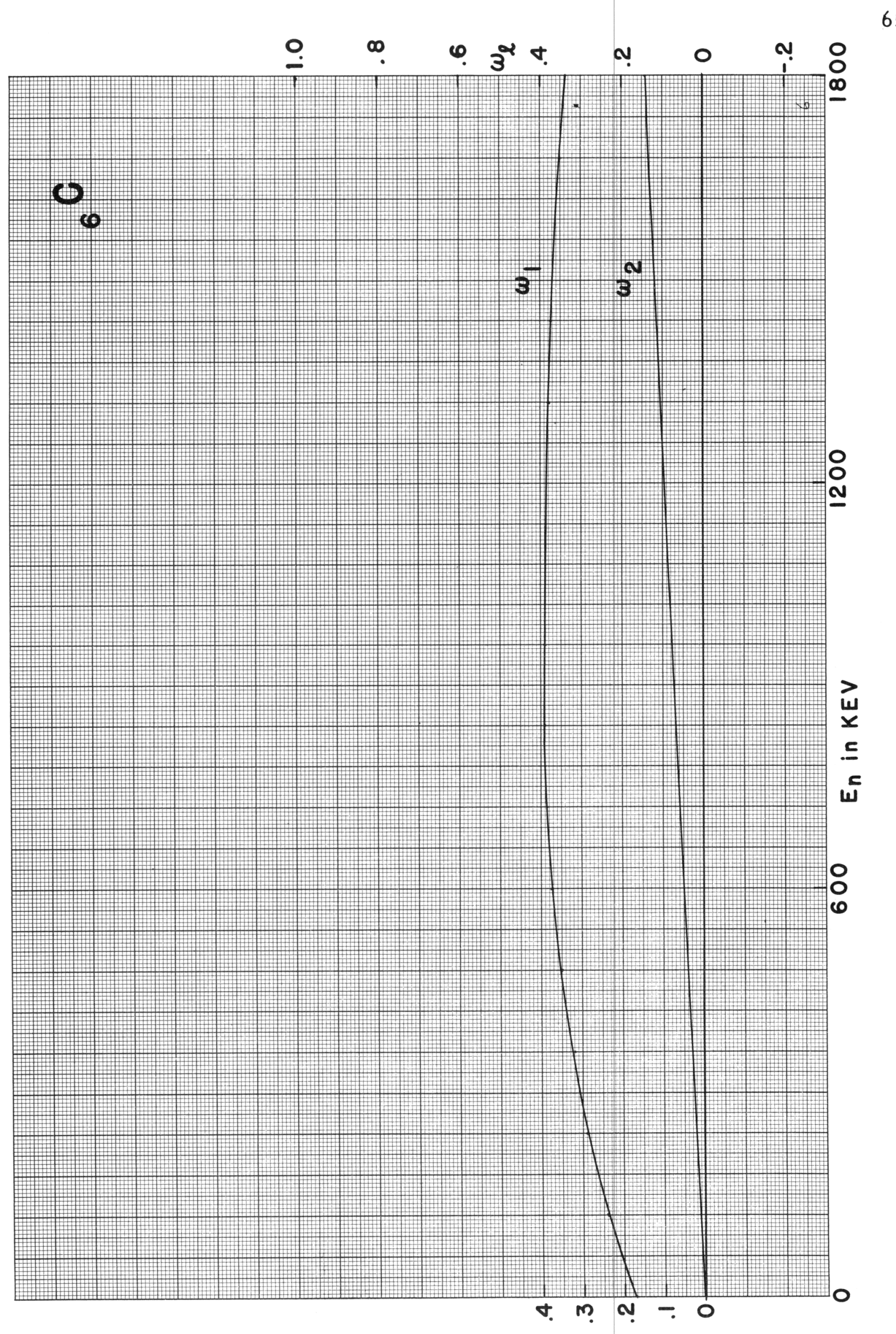




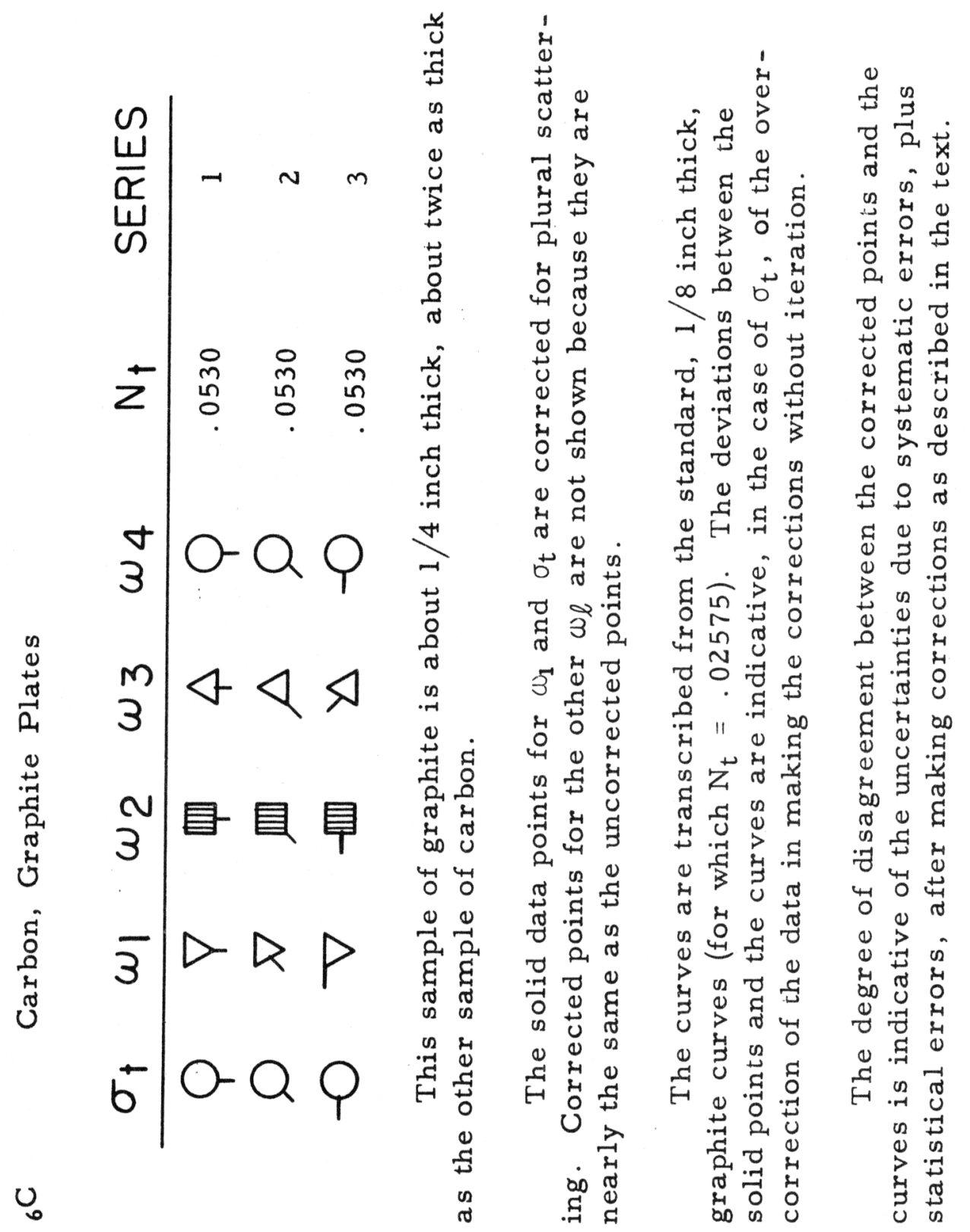




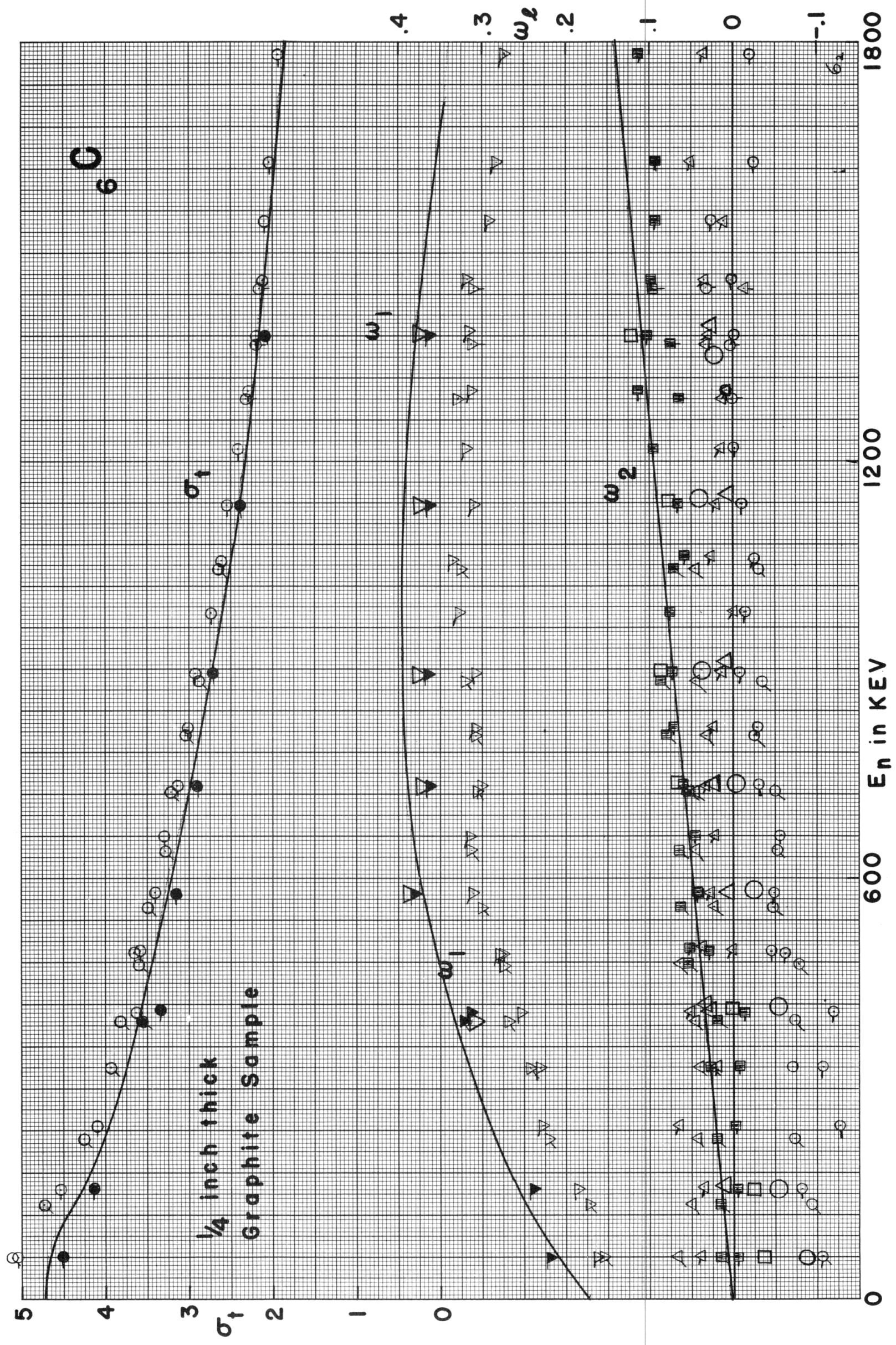




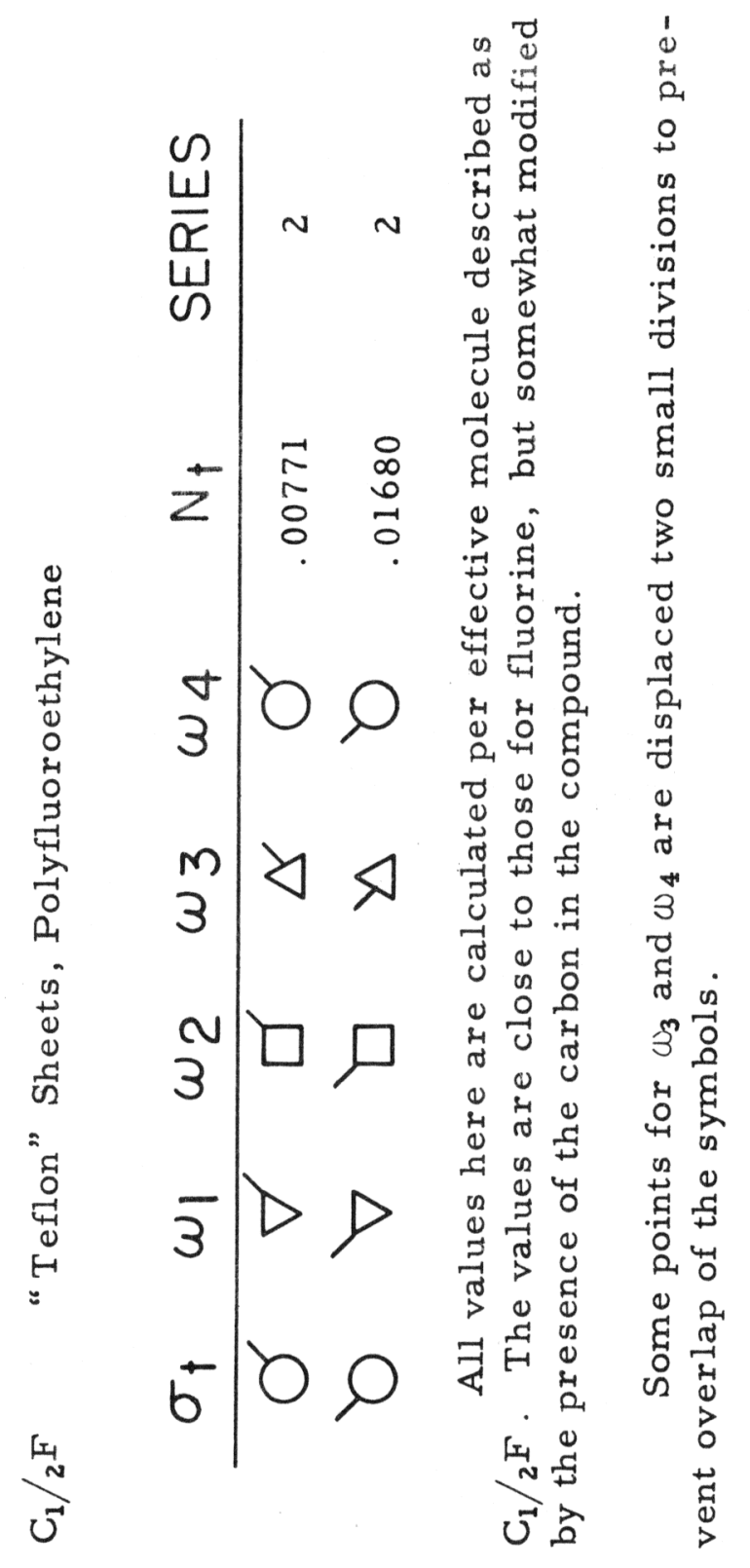




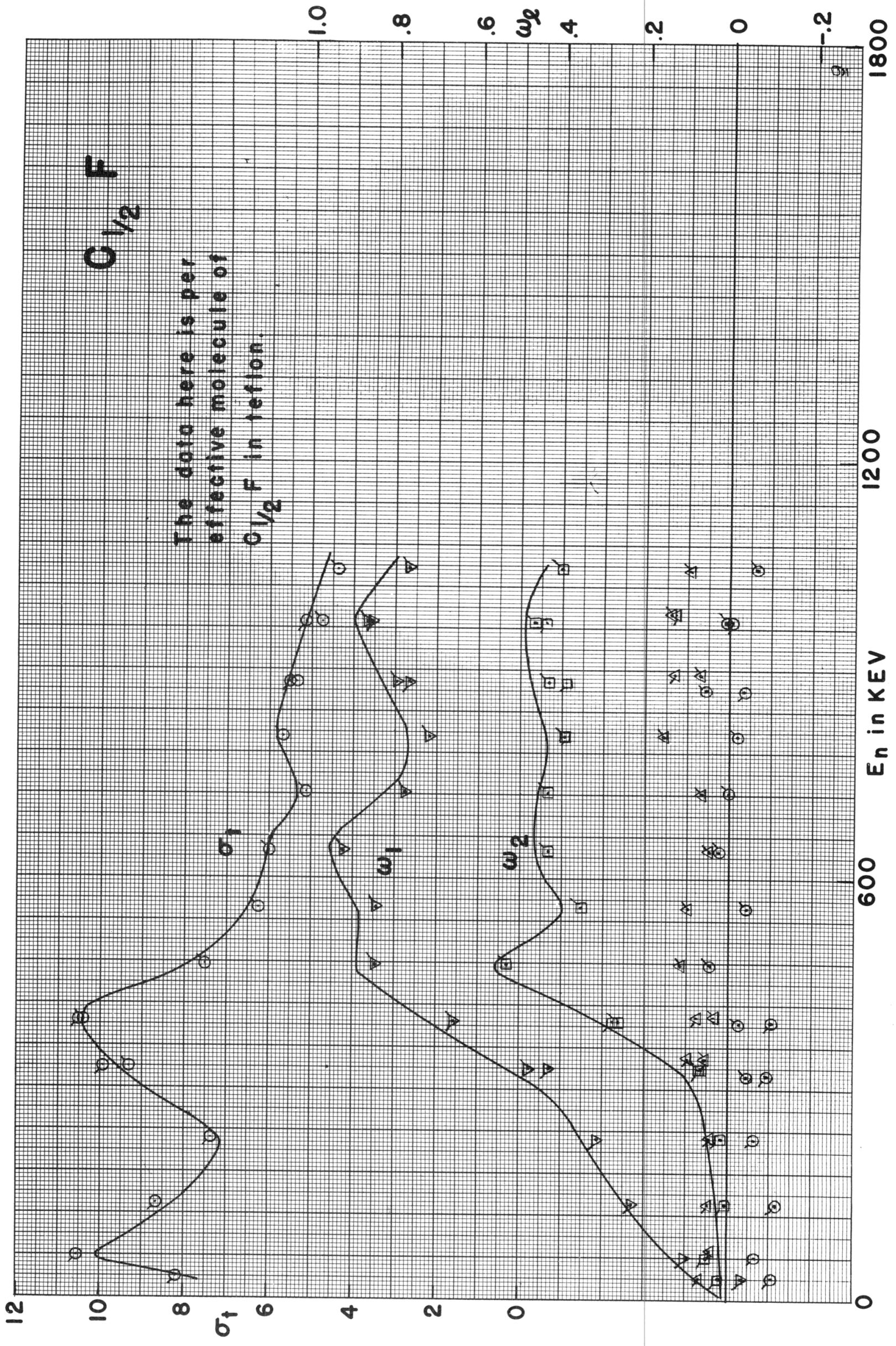




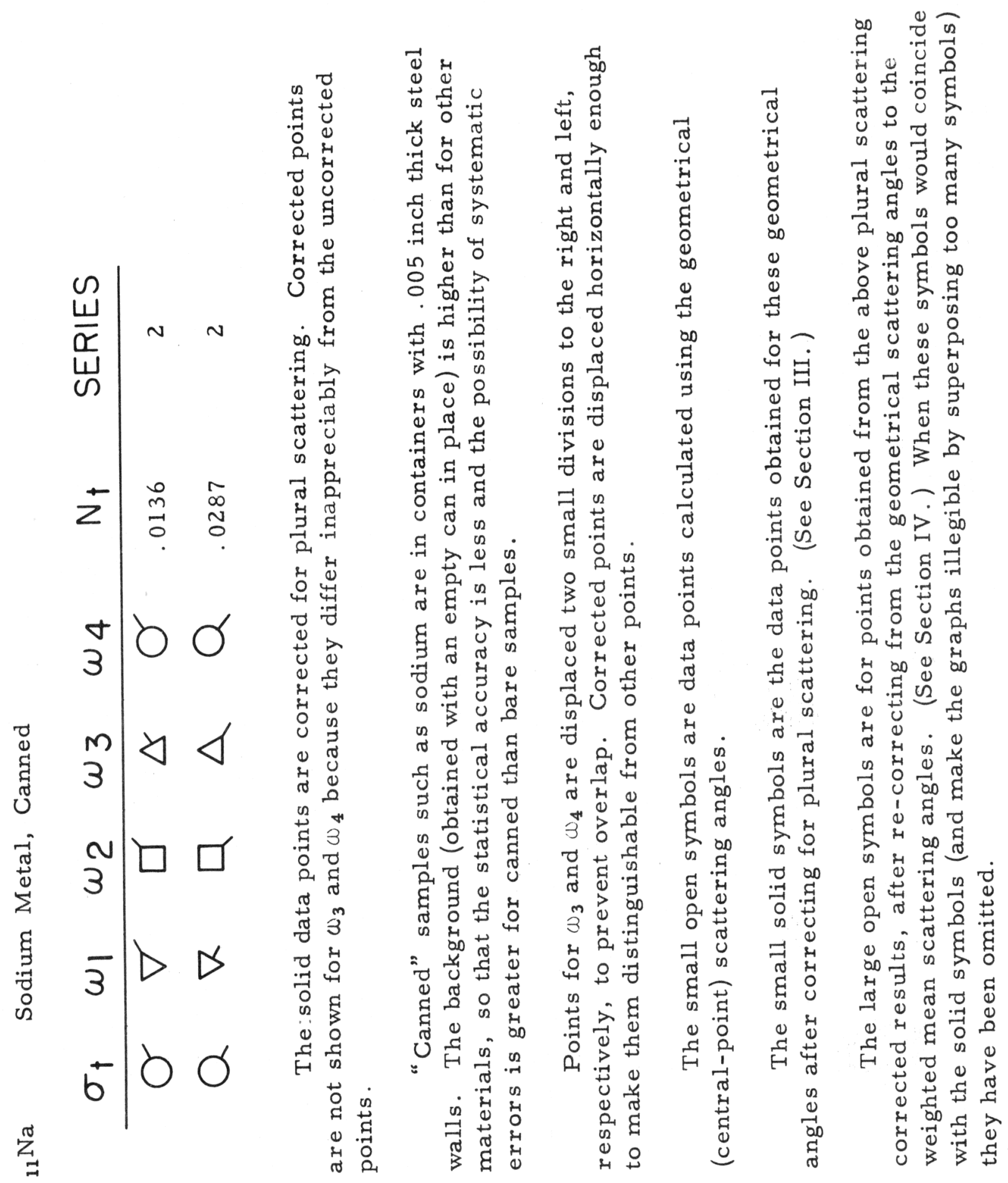




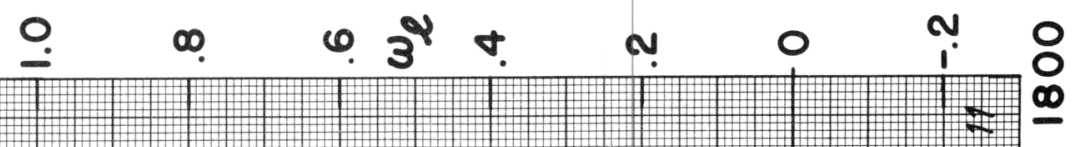

\section{$i^{i}$}

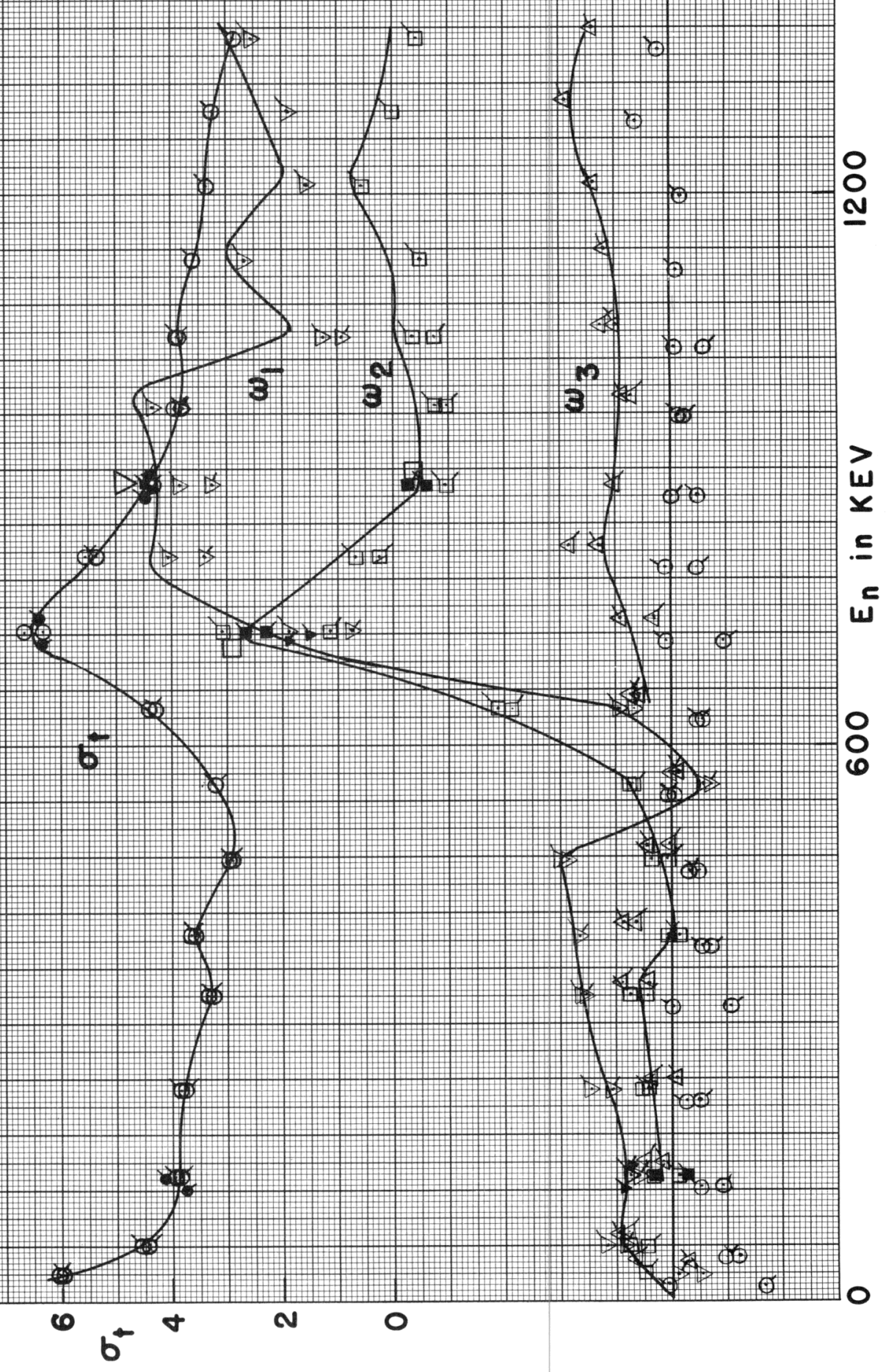




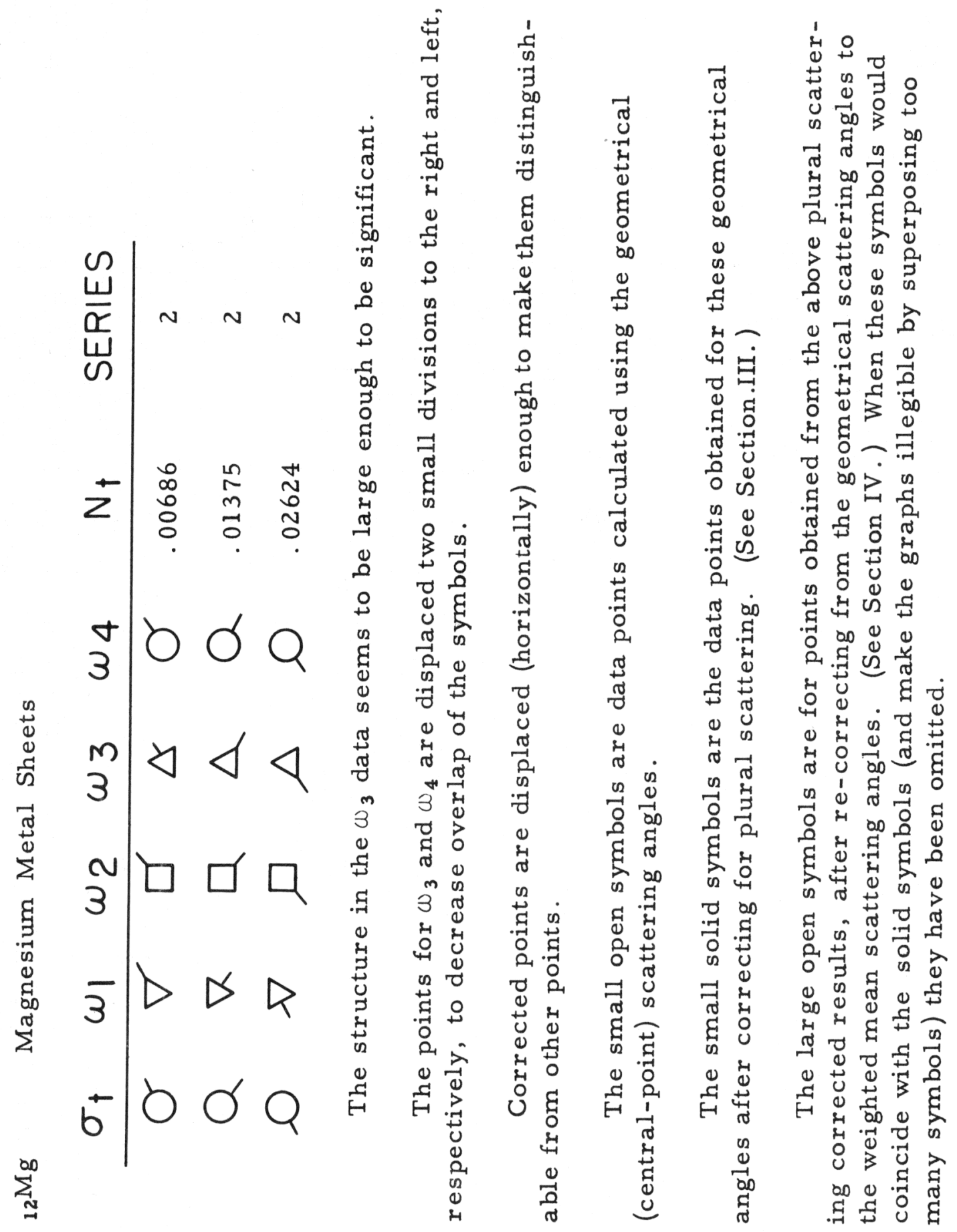




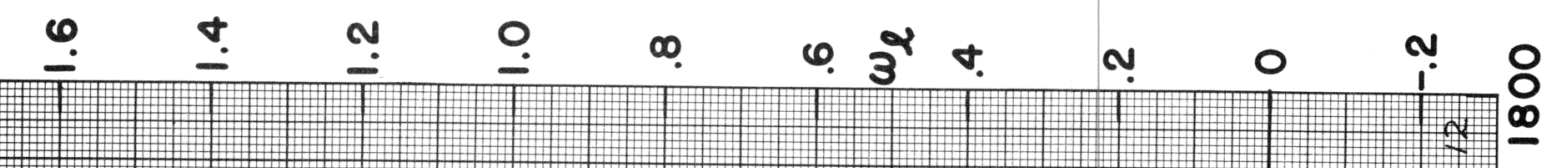
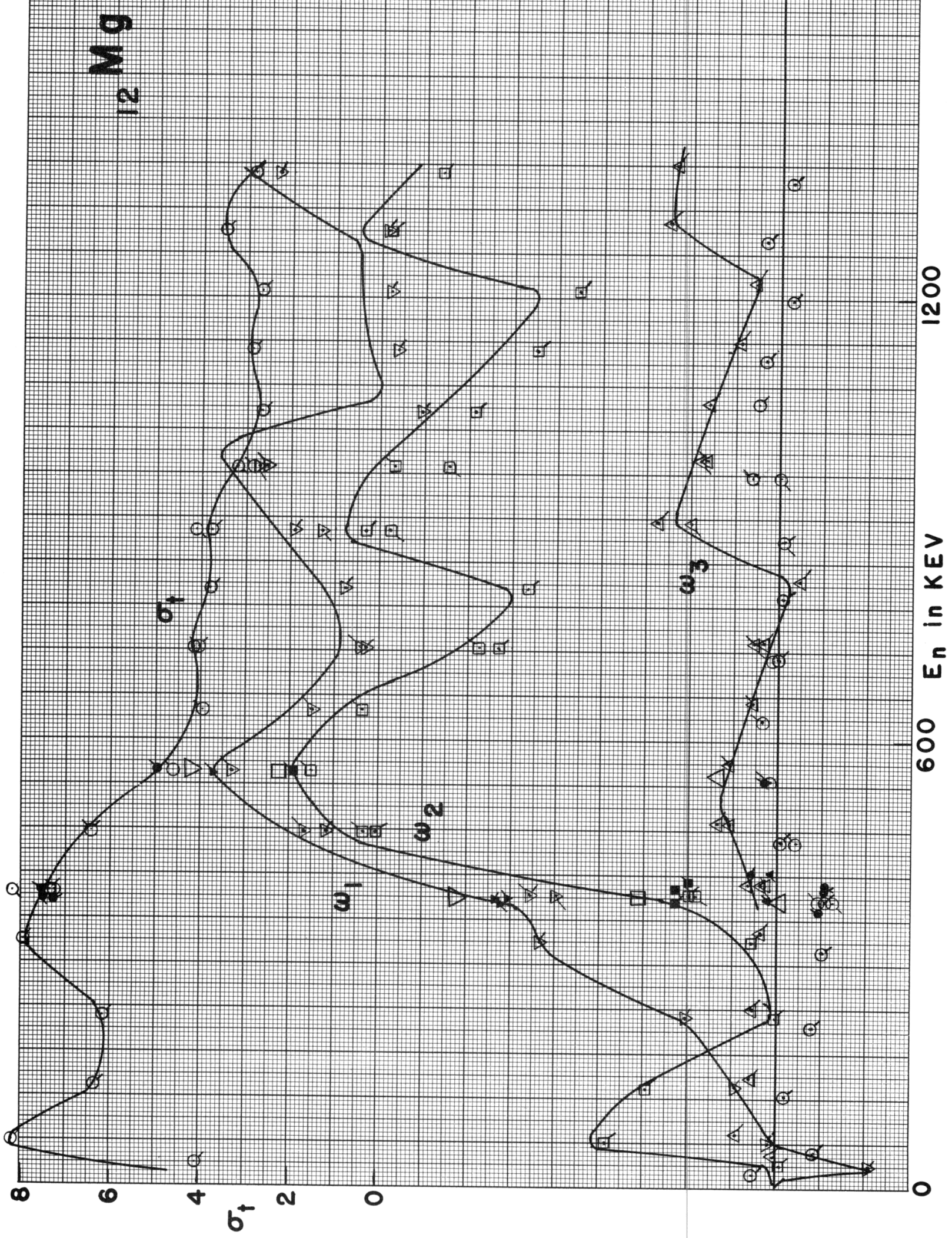


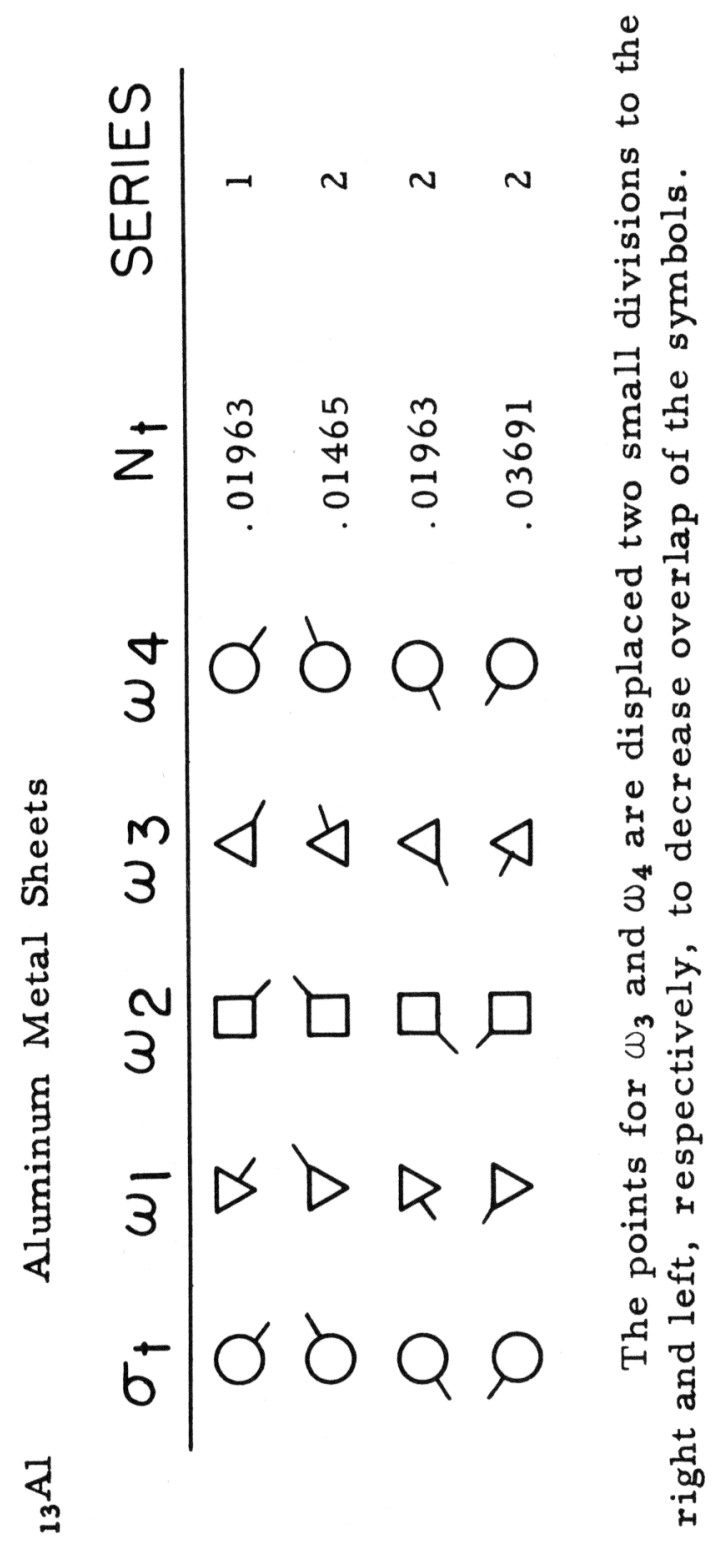




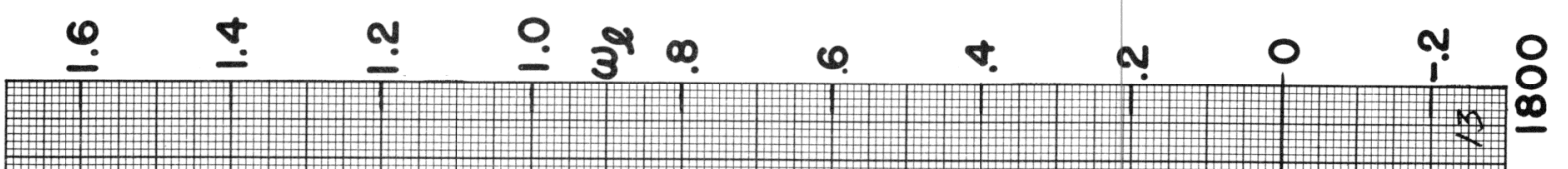
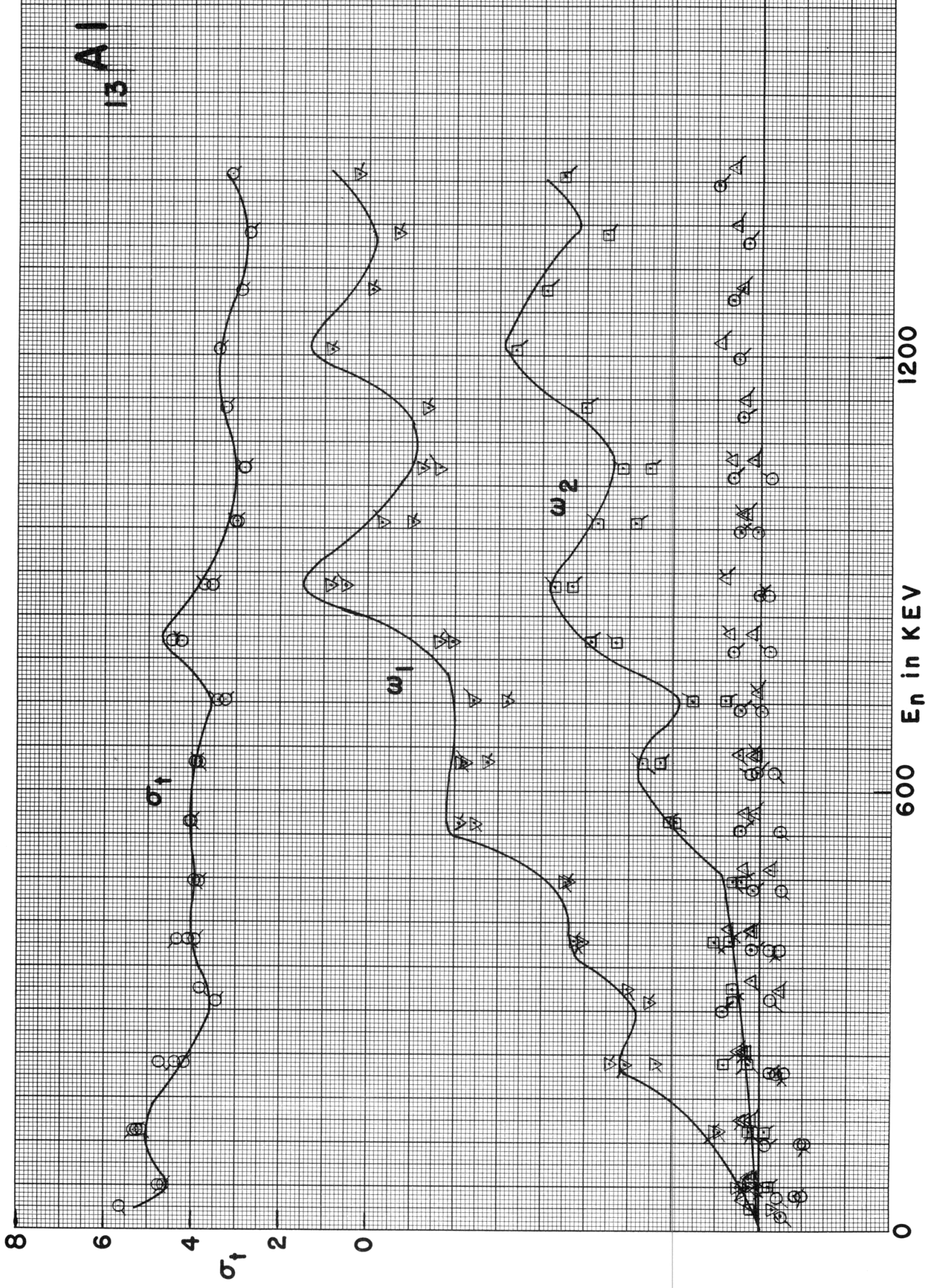


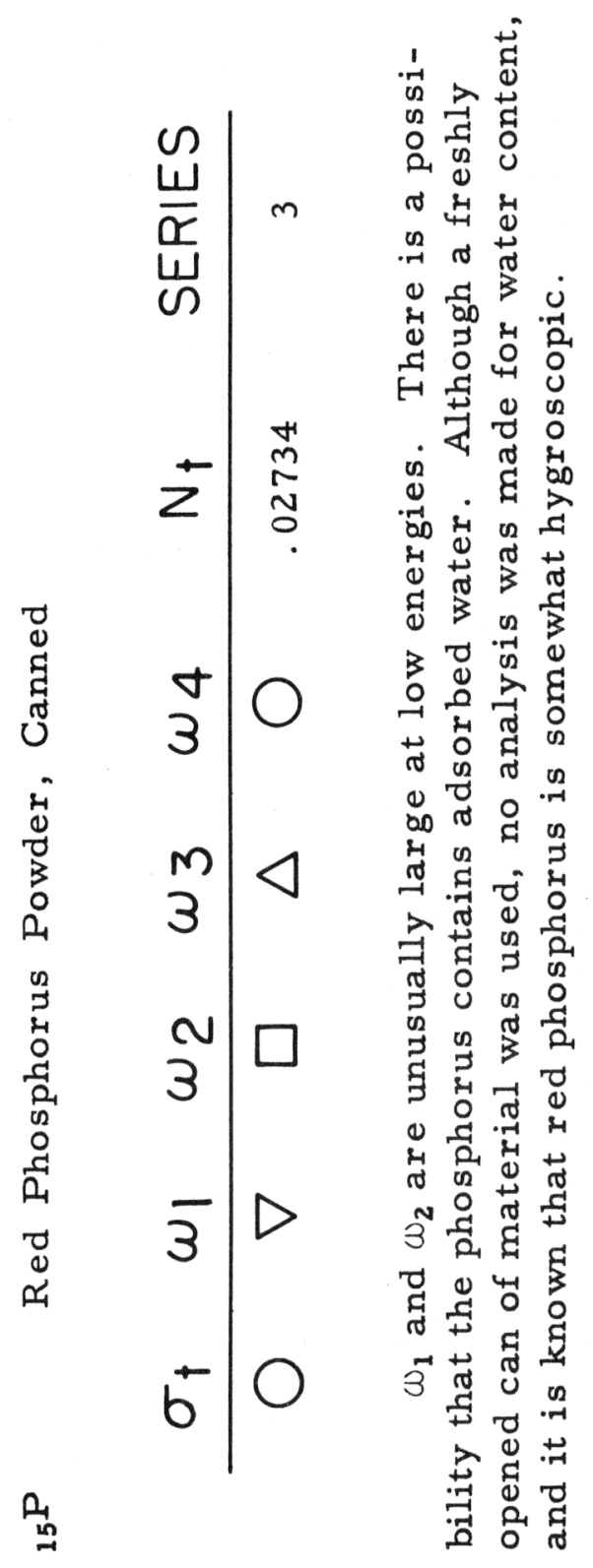




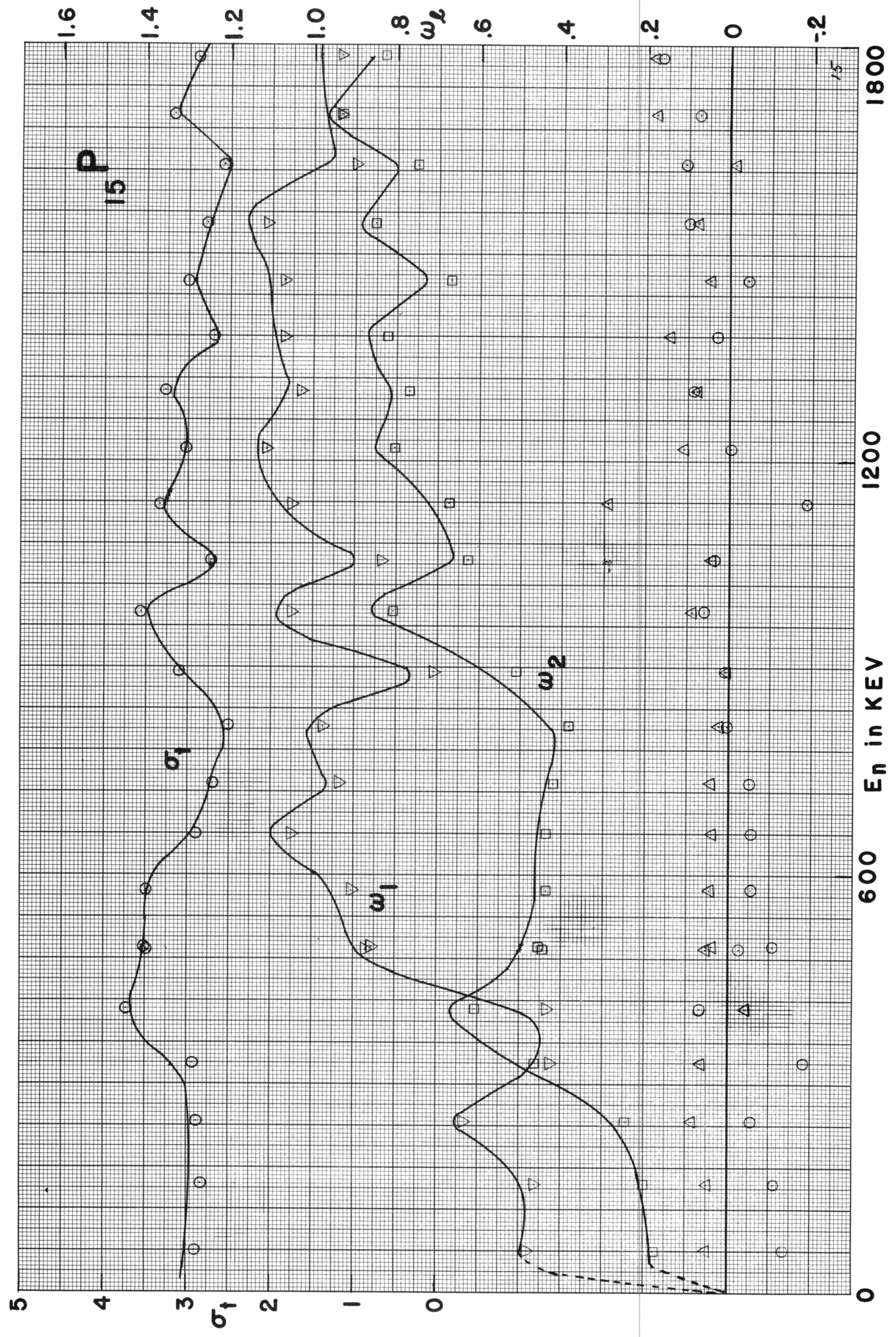




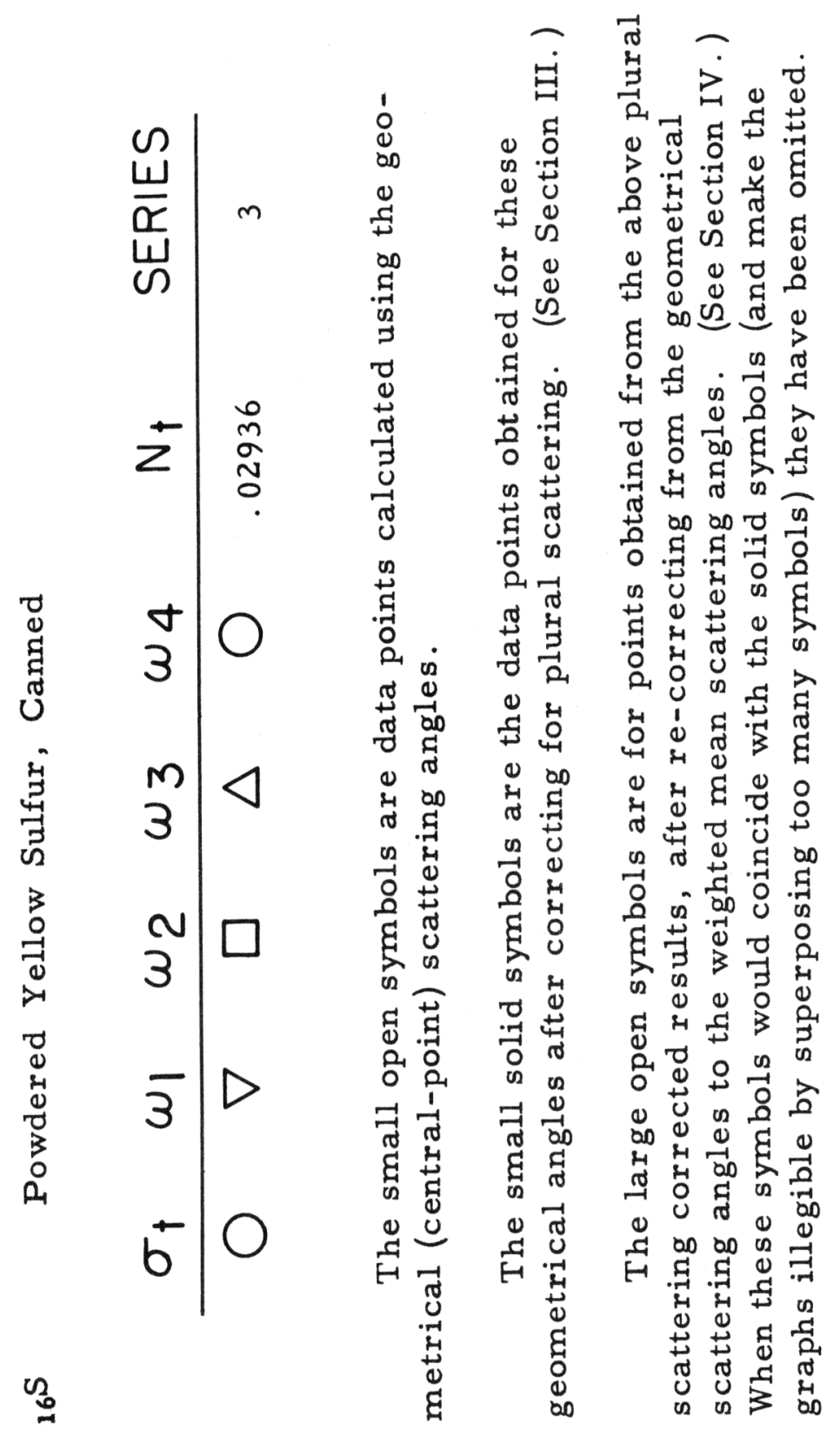




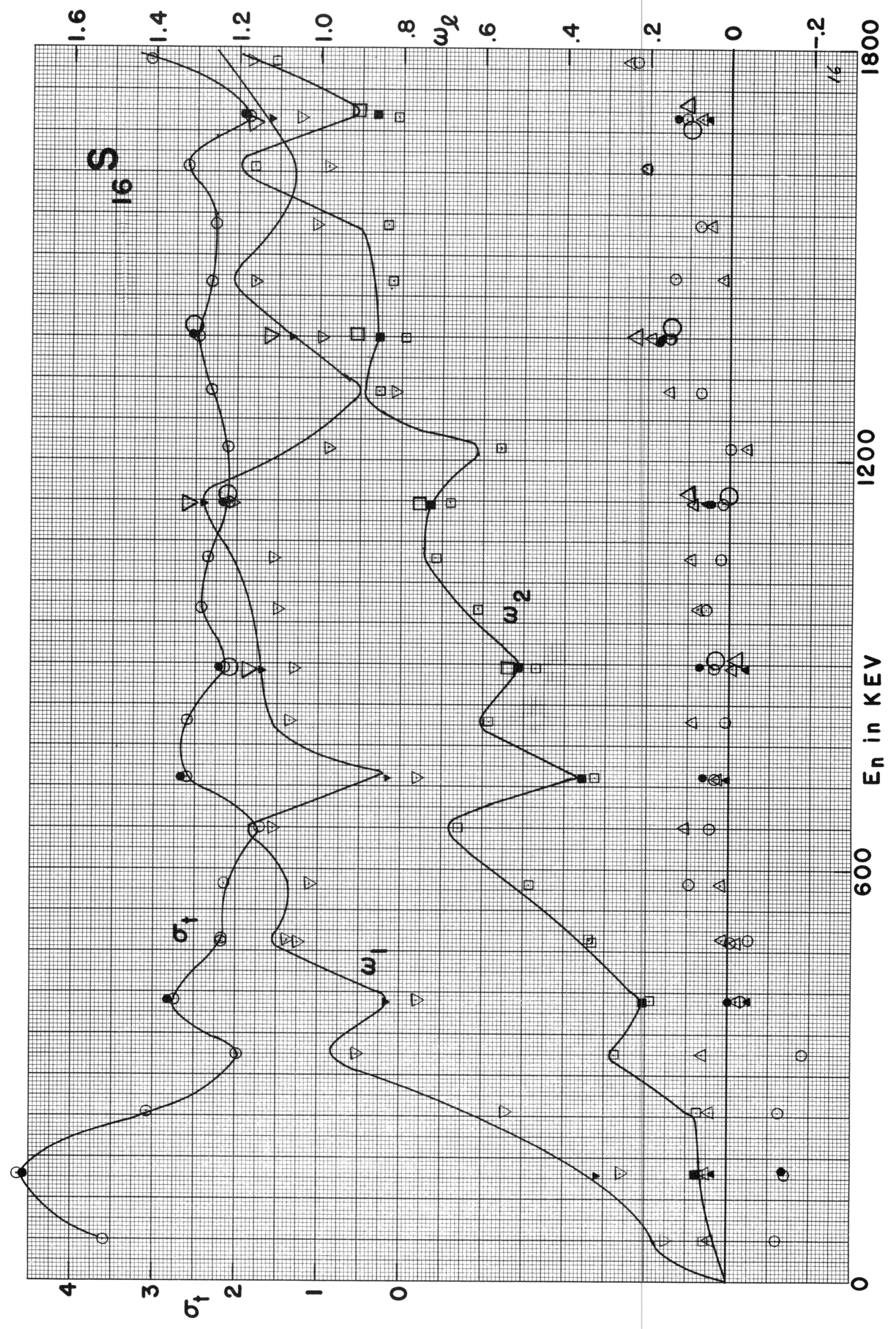




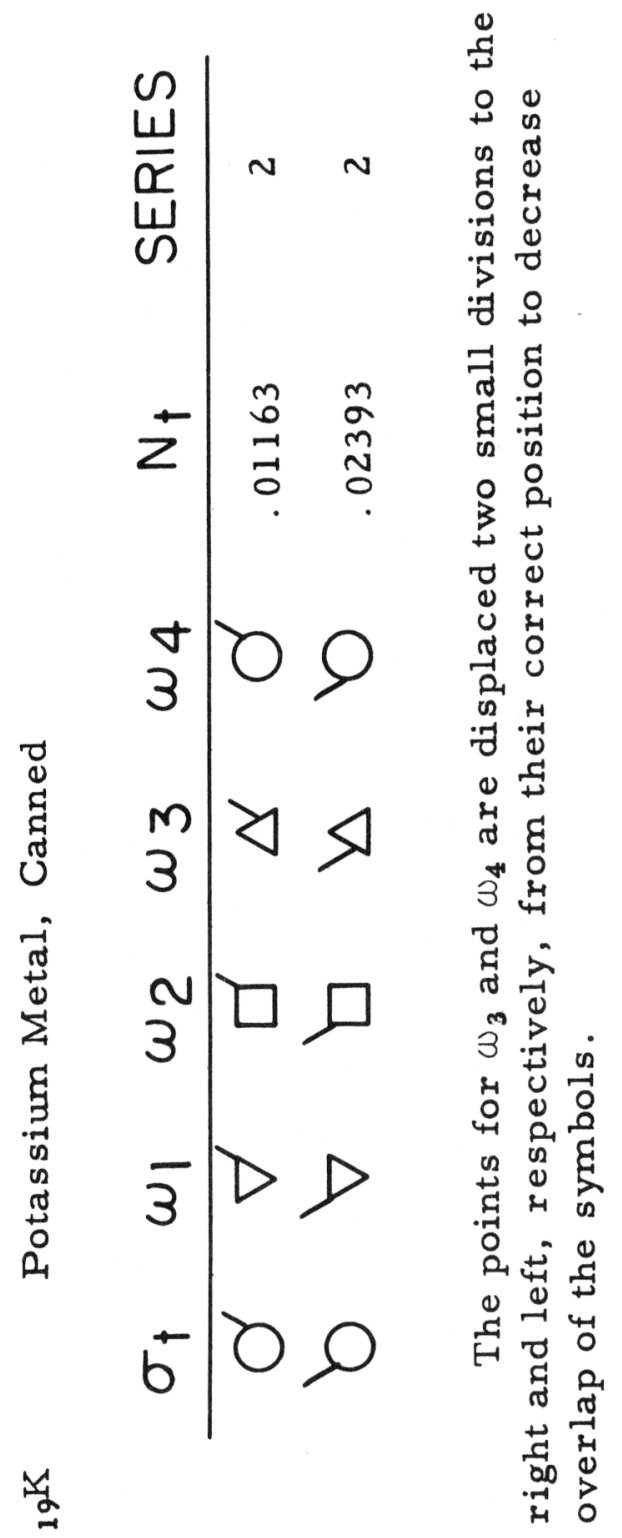




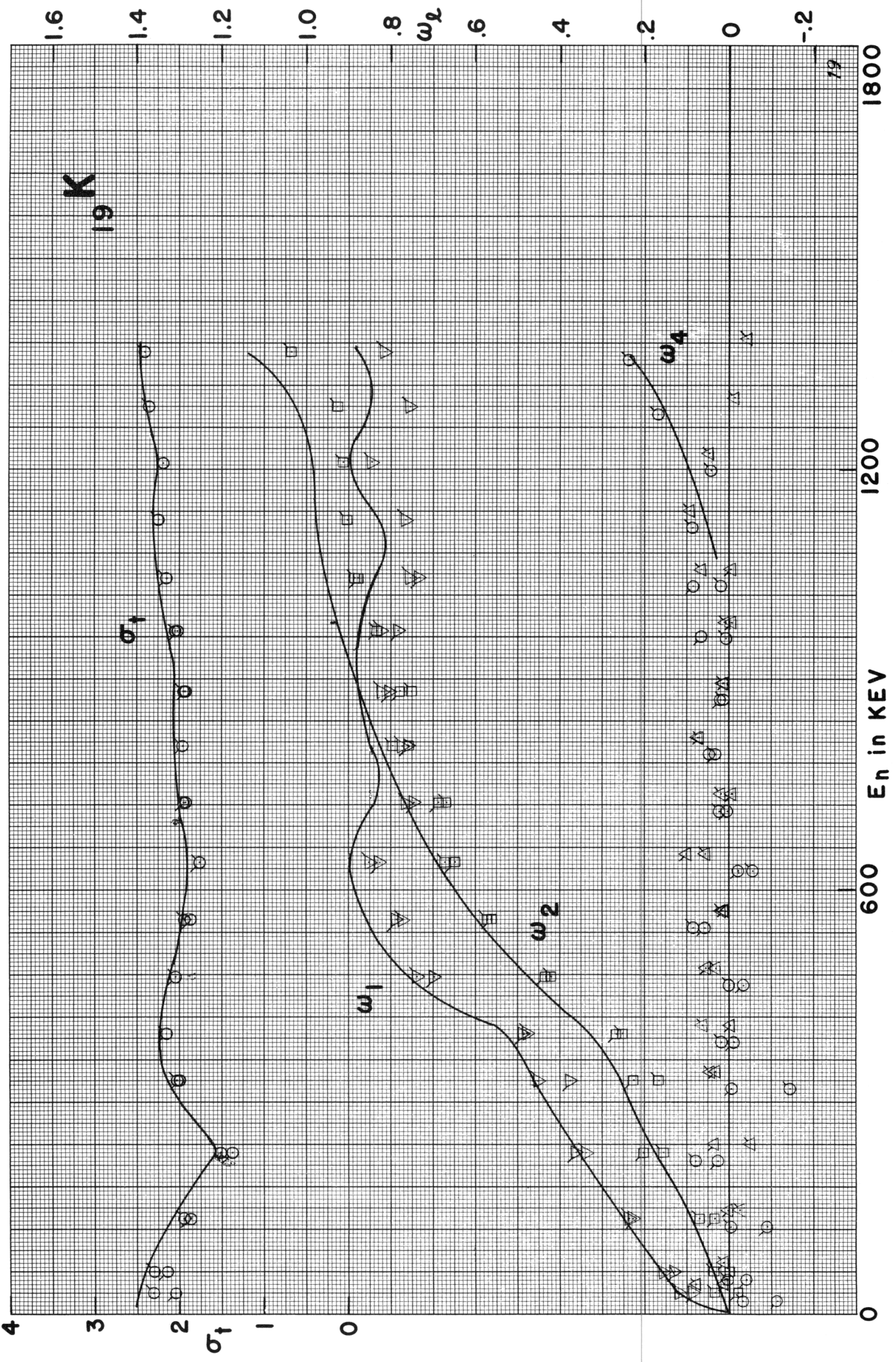




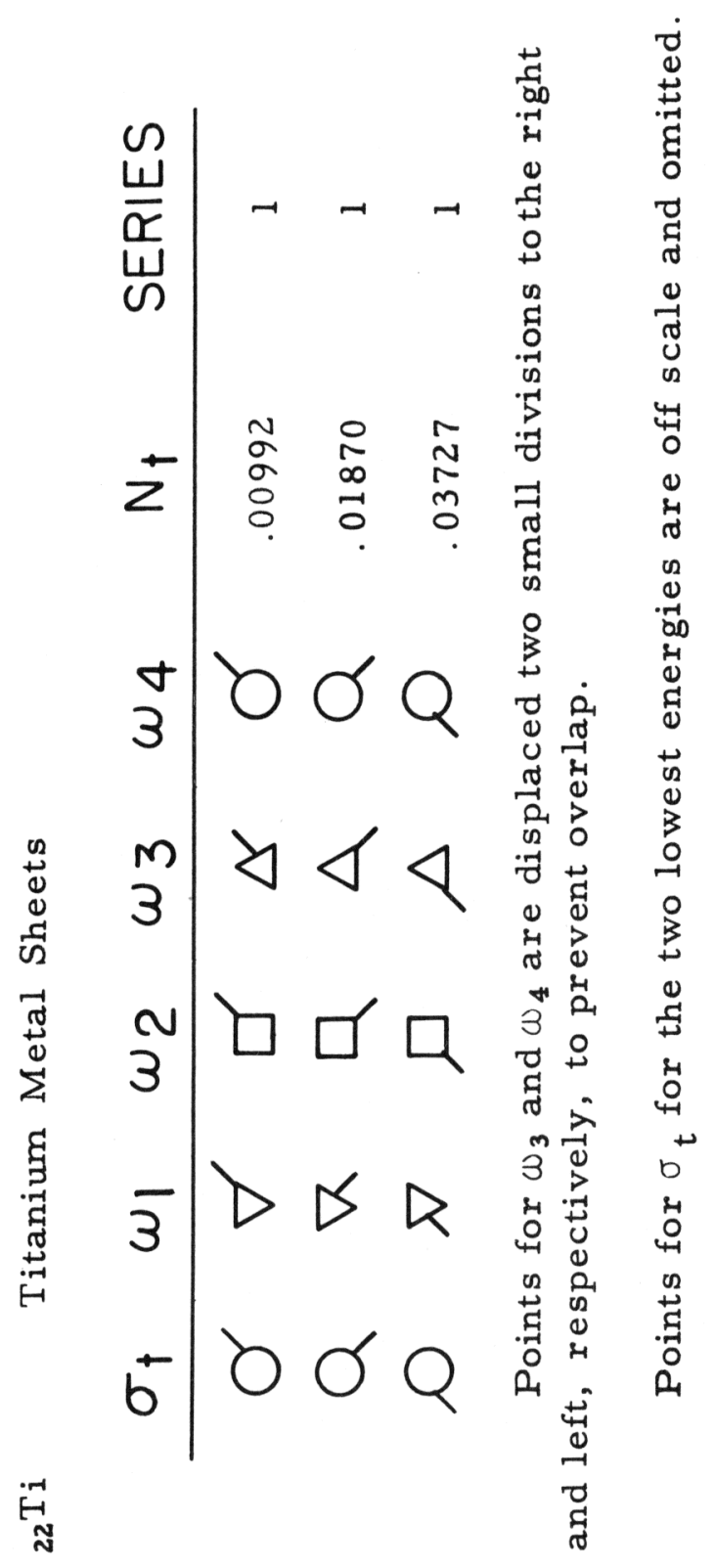




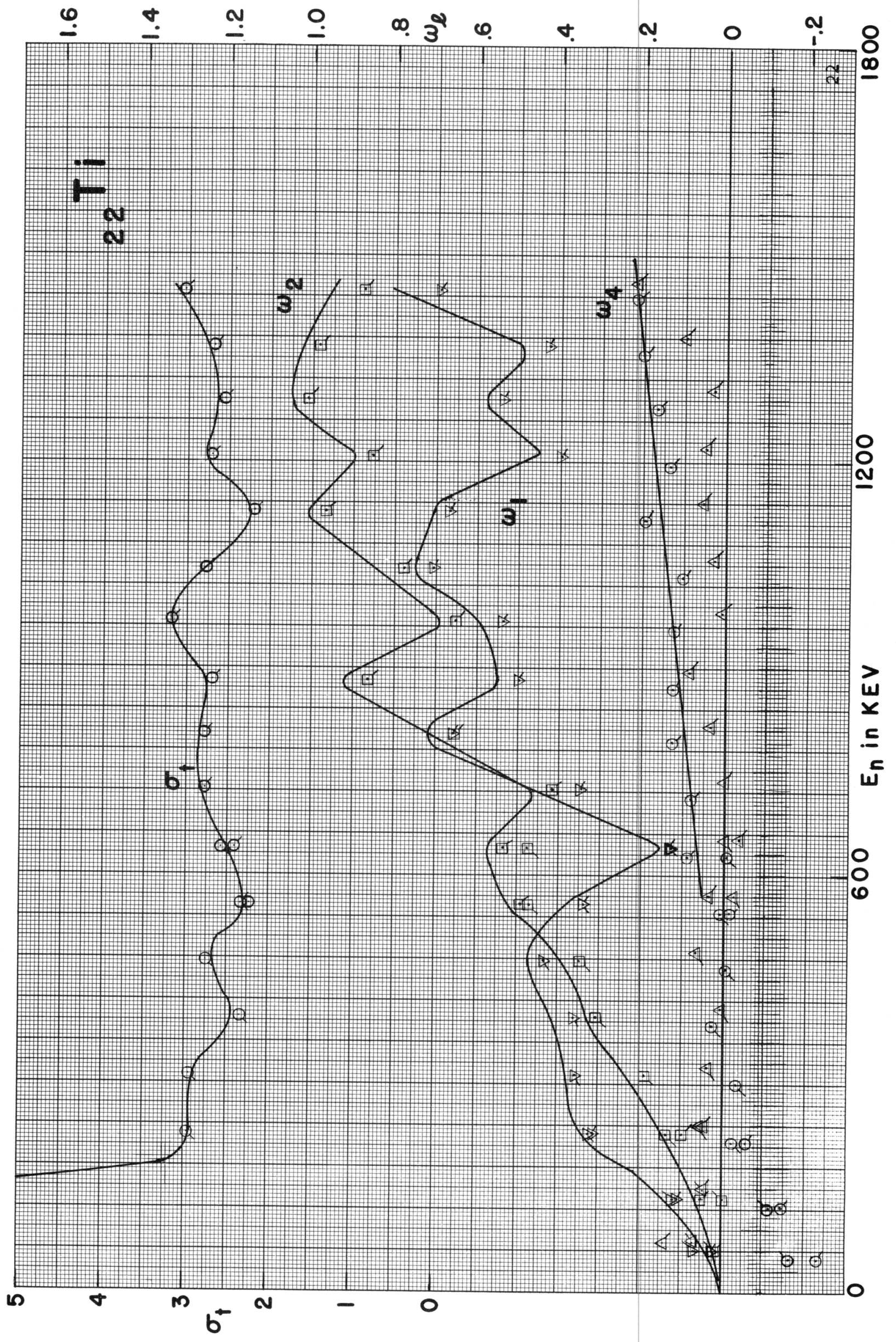




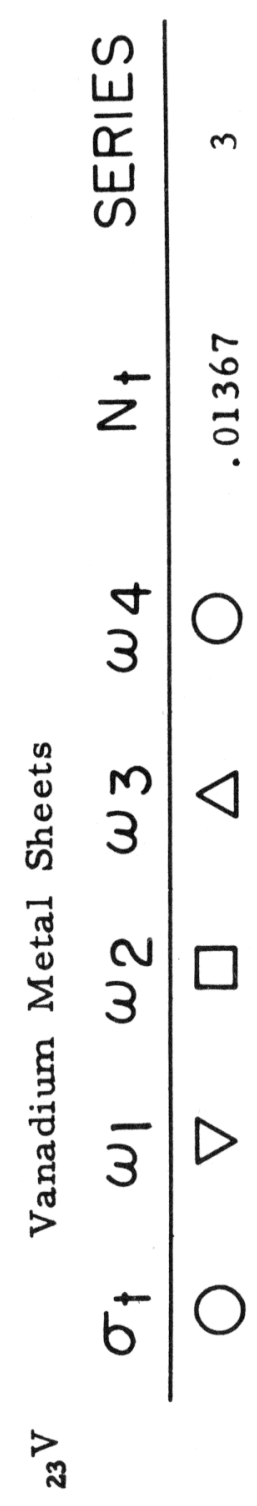




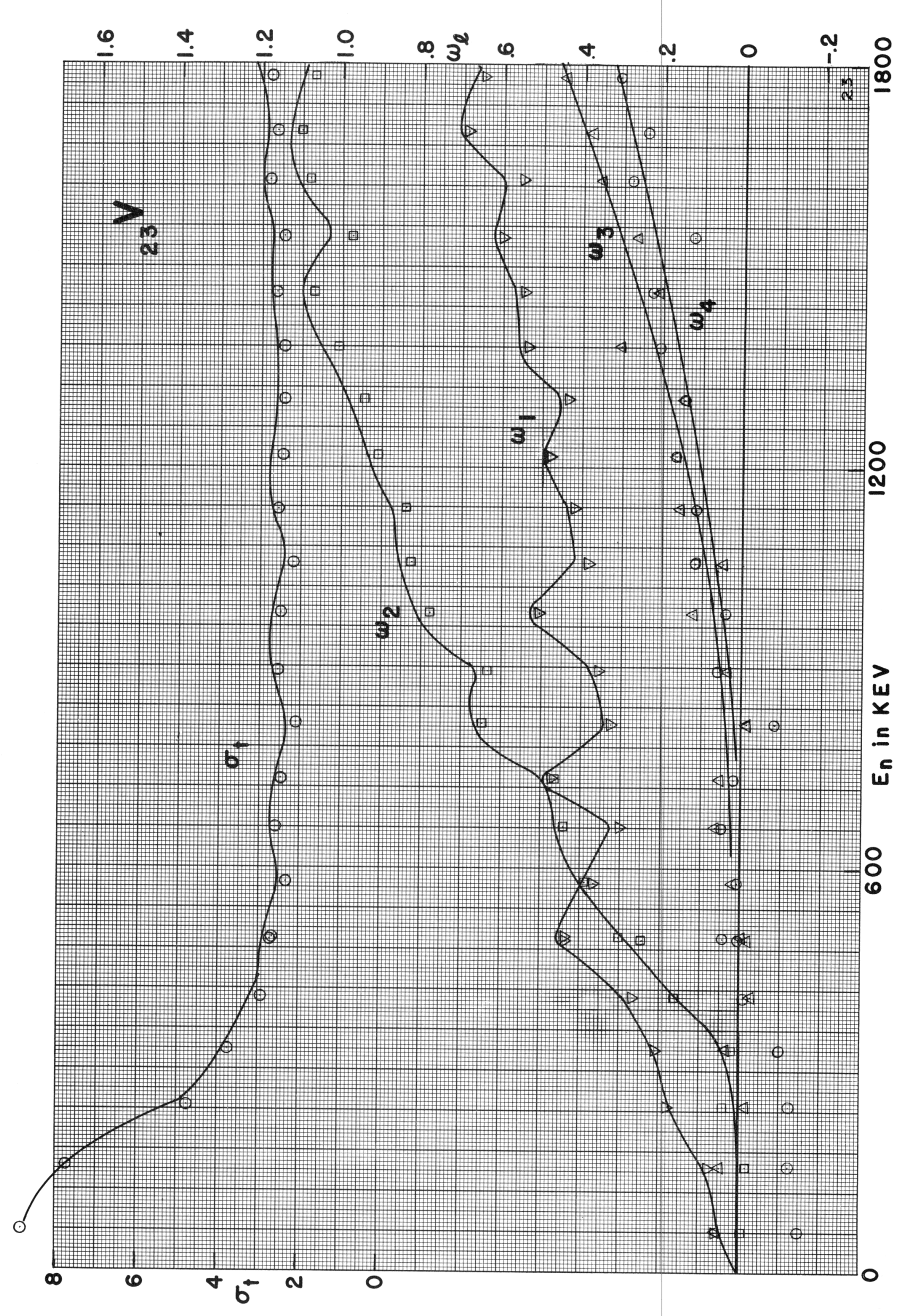




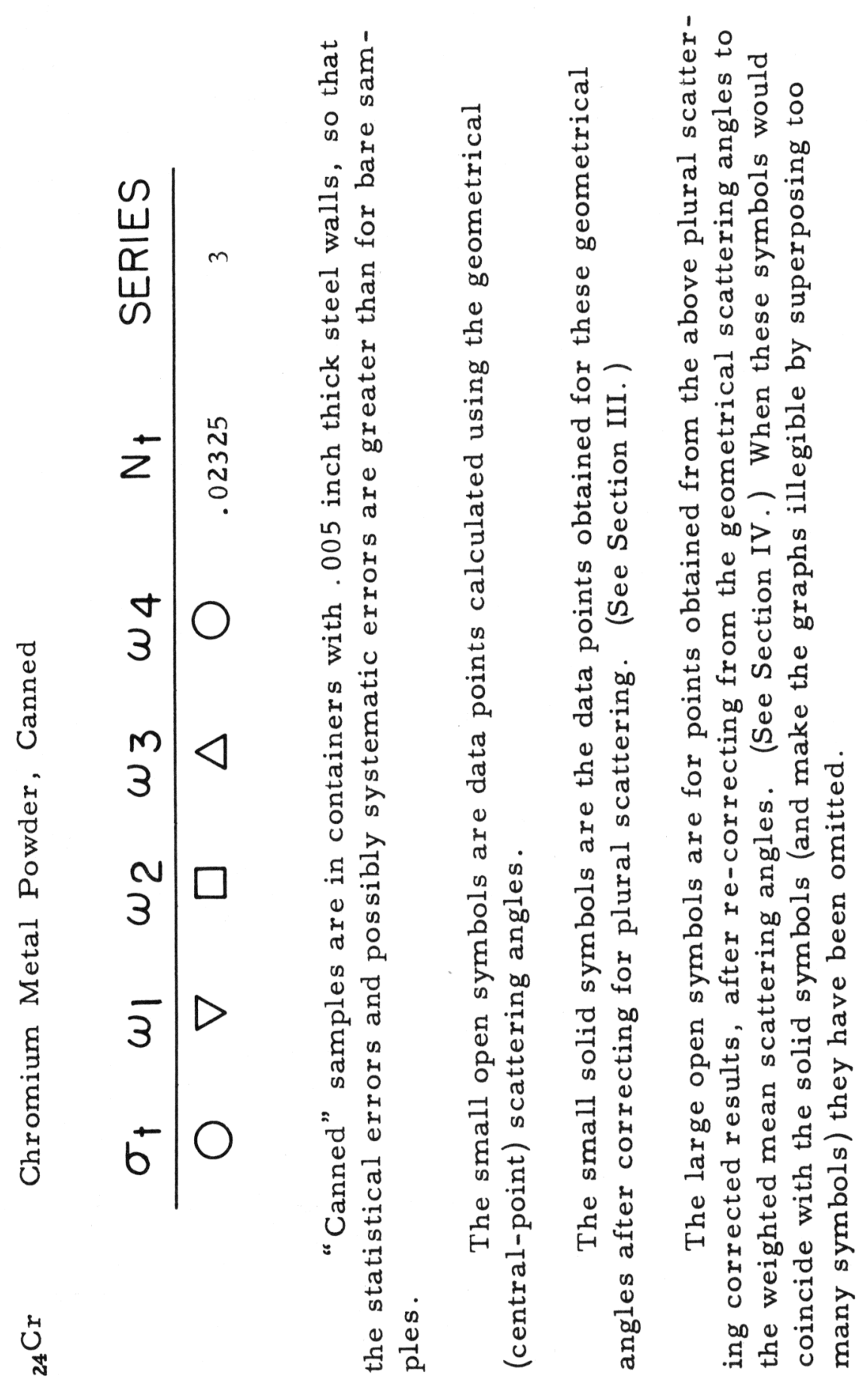




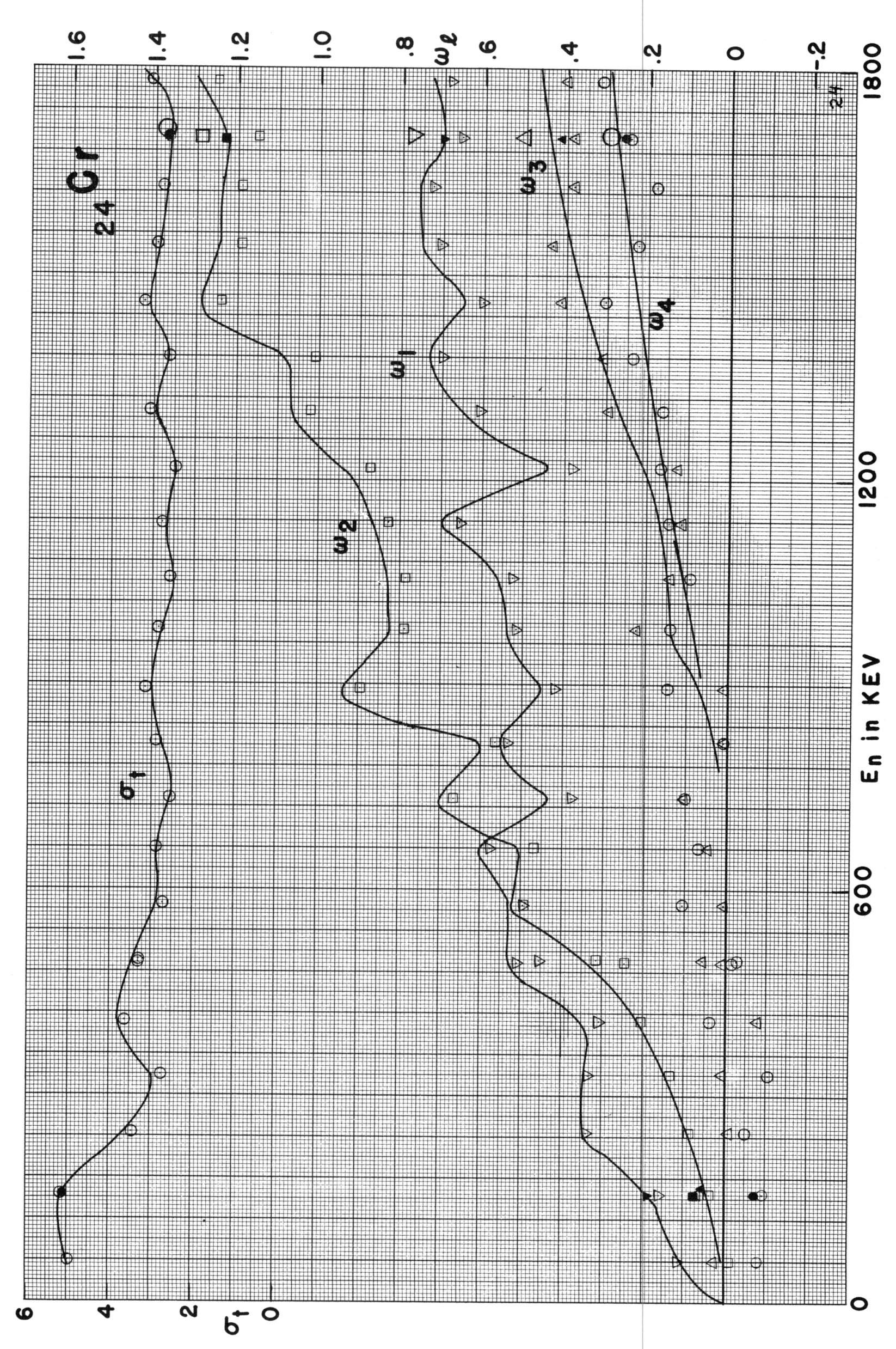



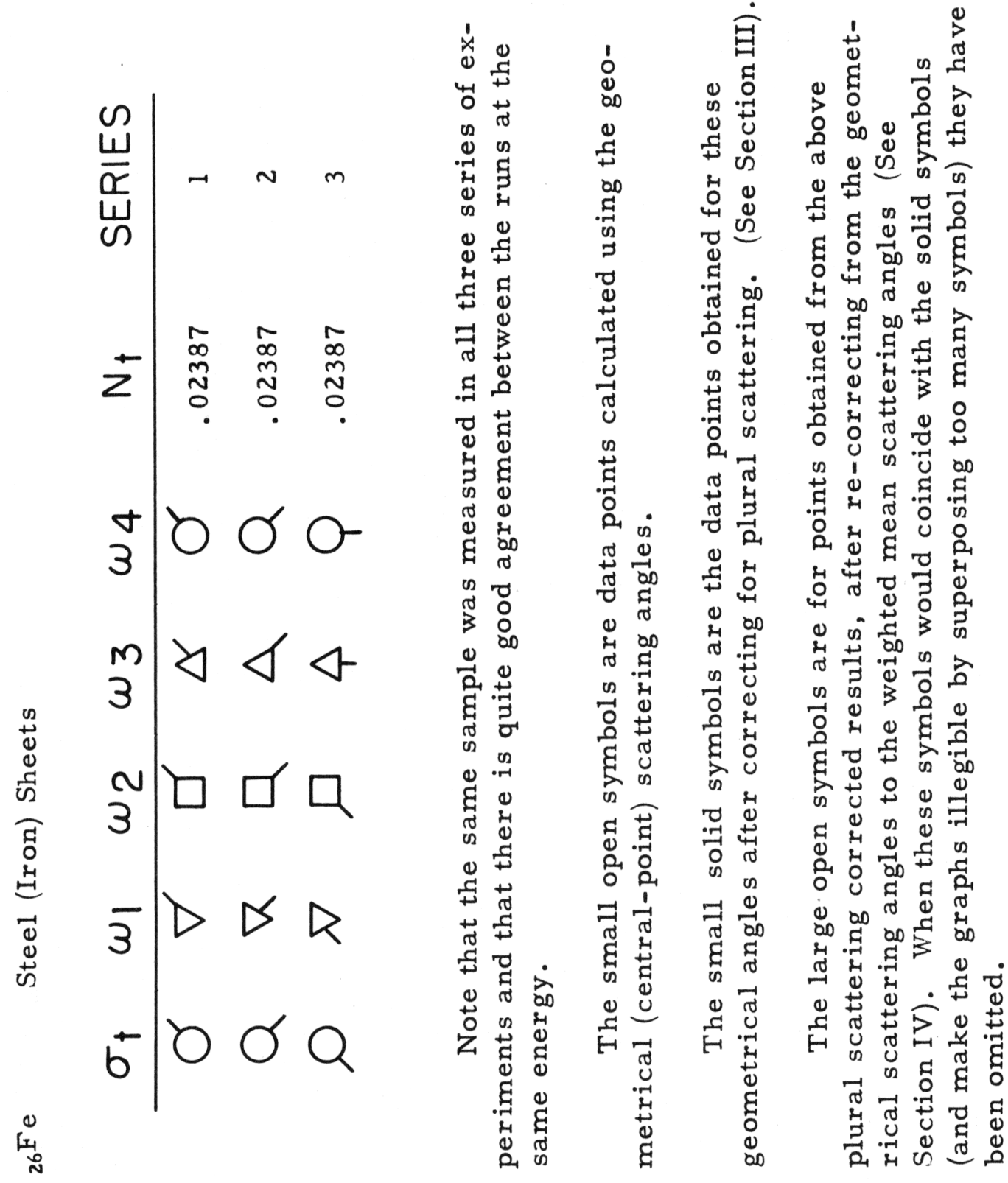


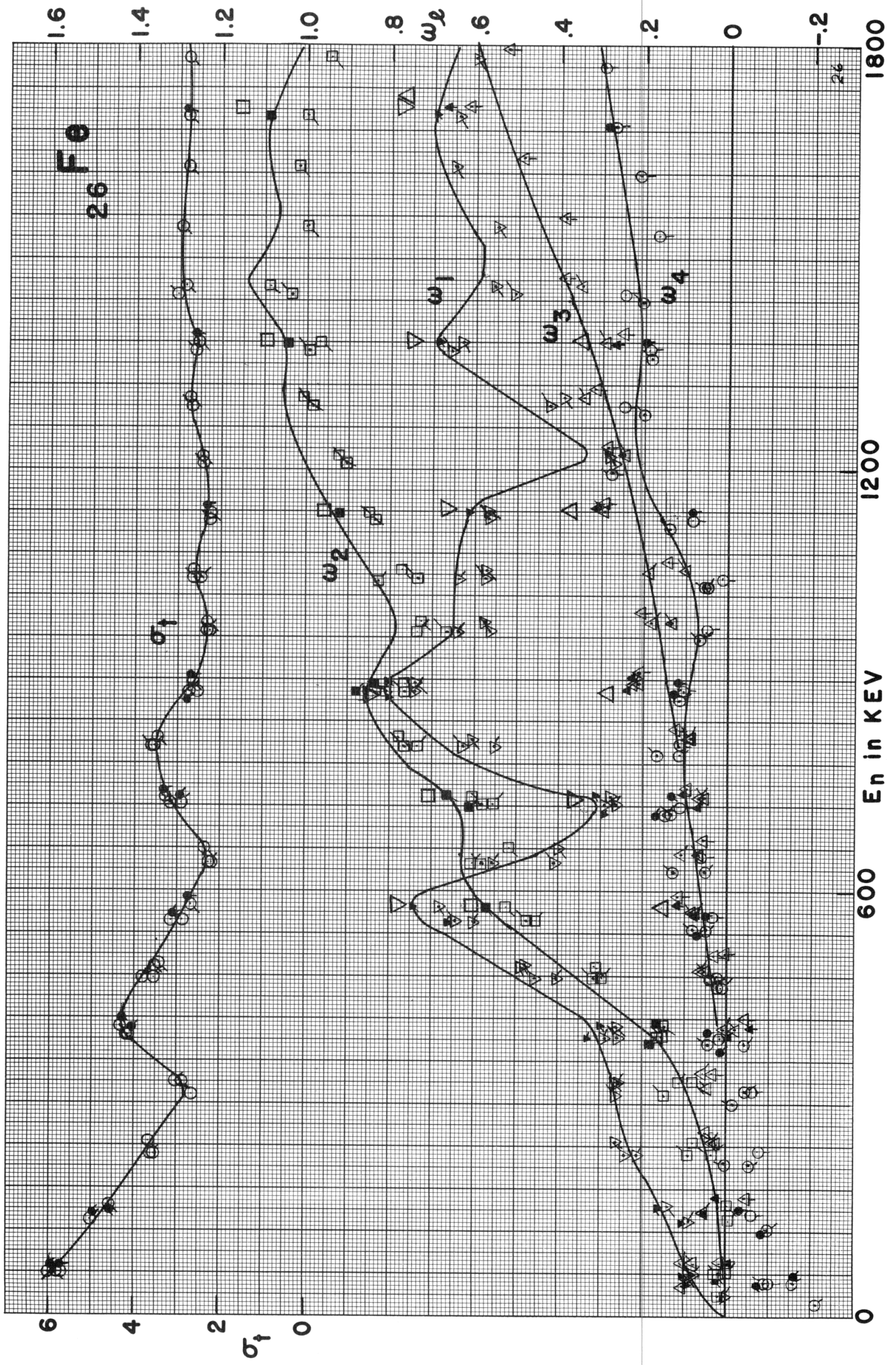




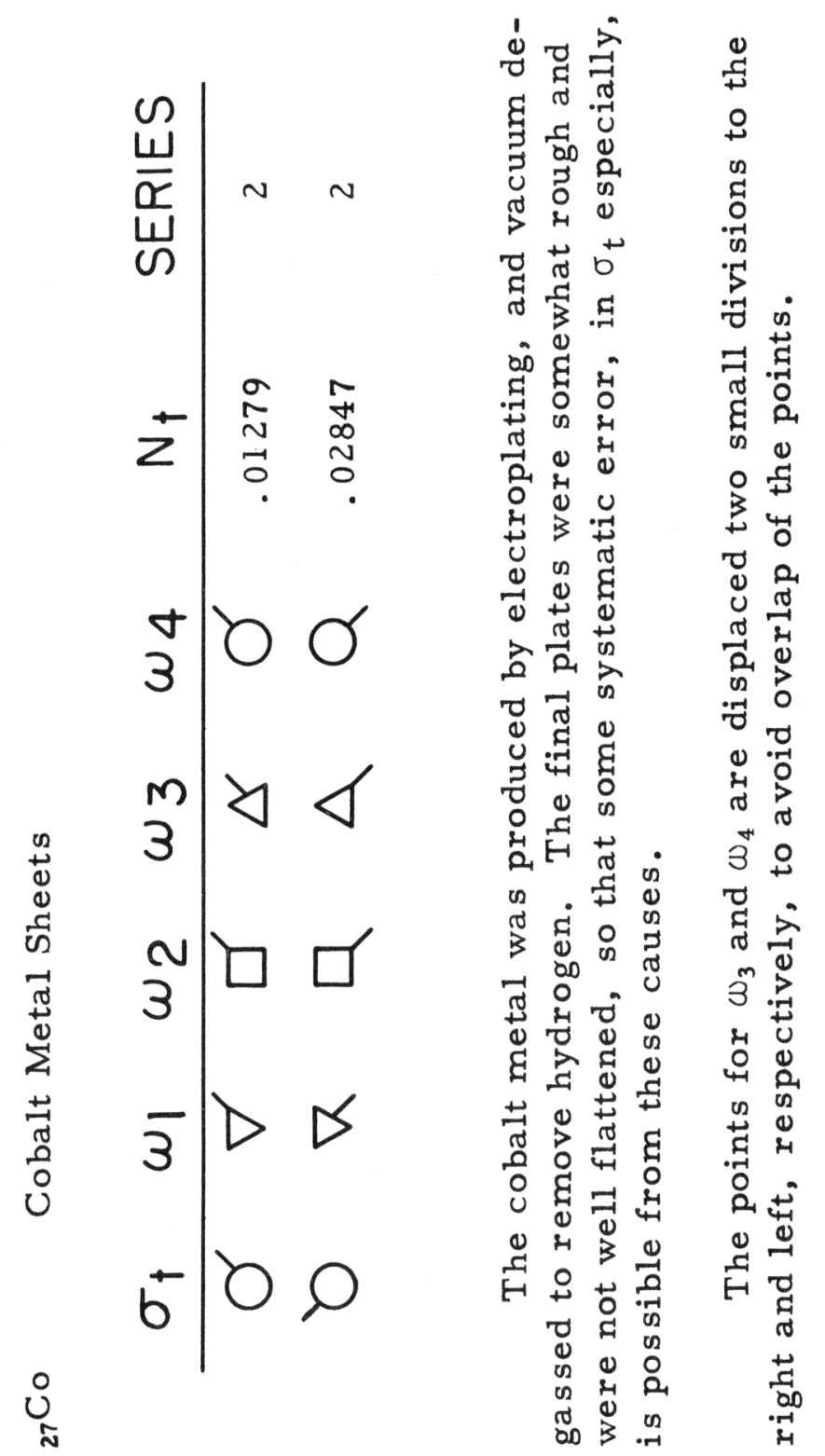




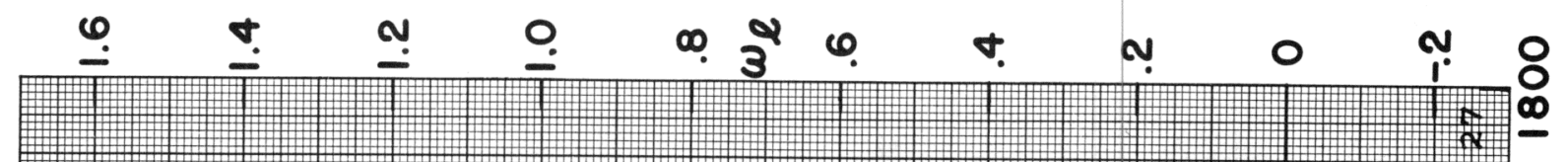

$\dot{0}_{\tilde{N}}$
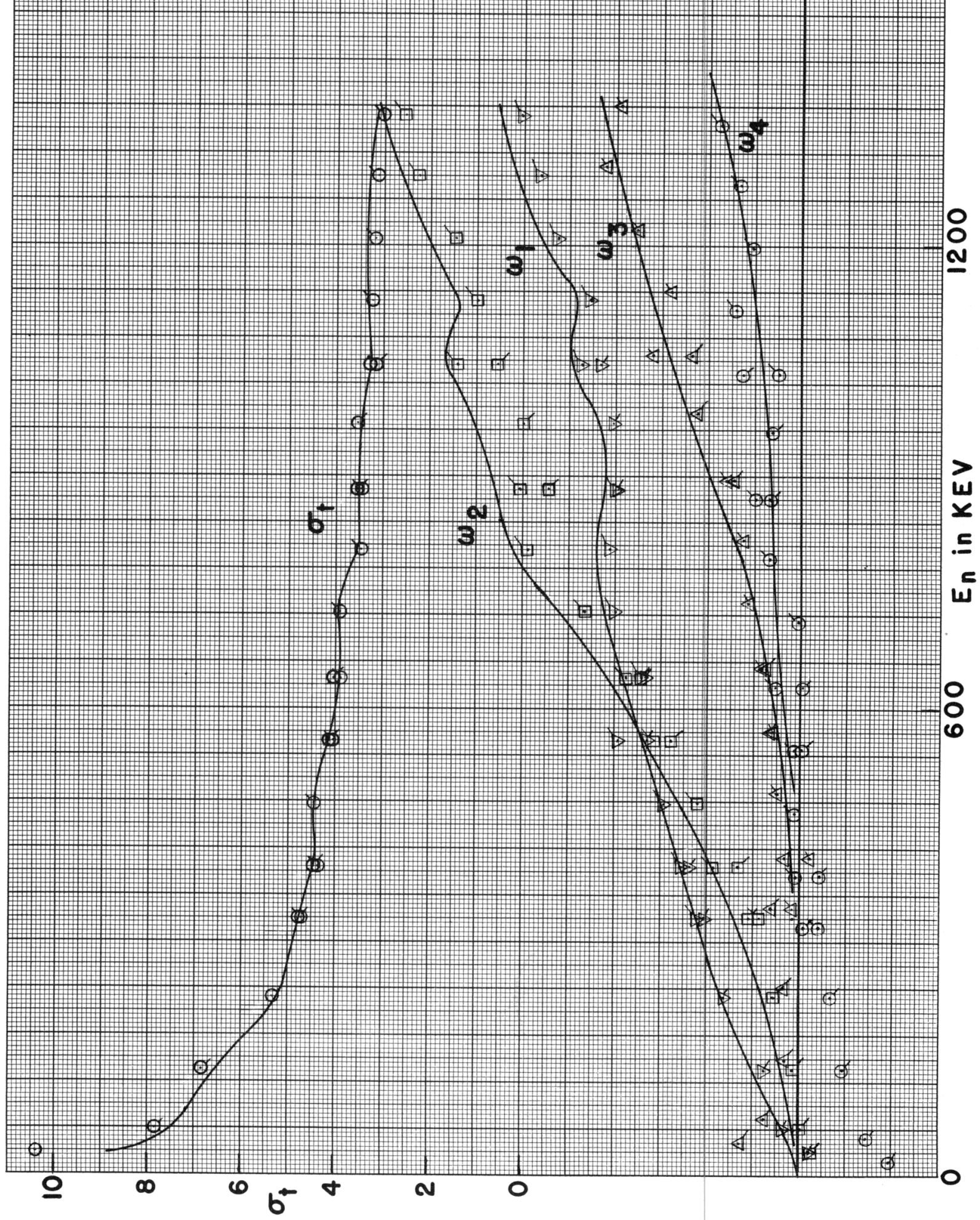


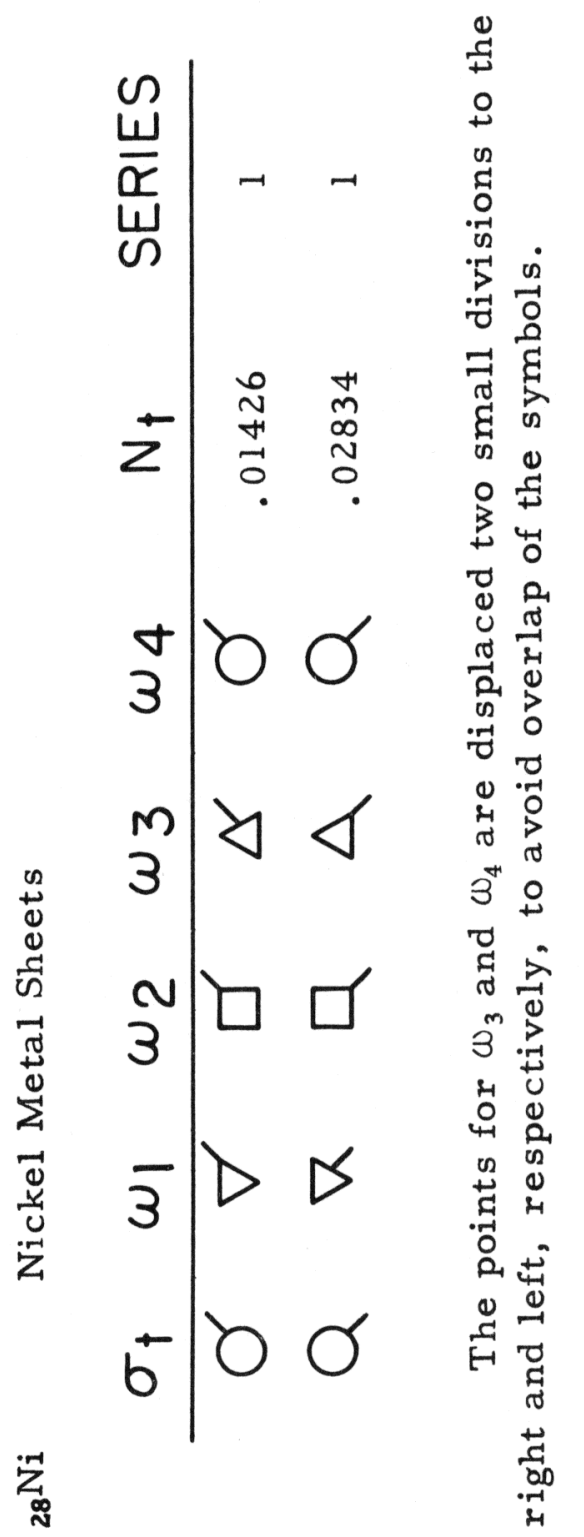




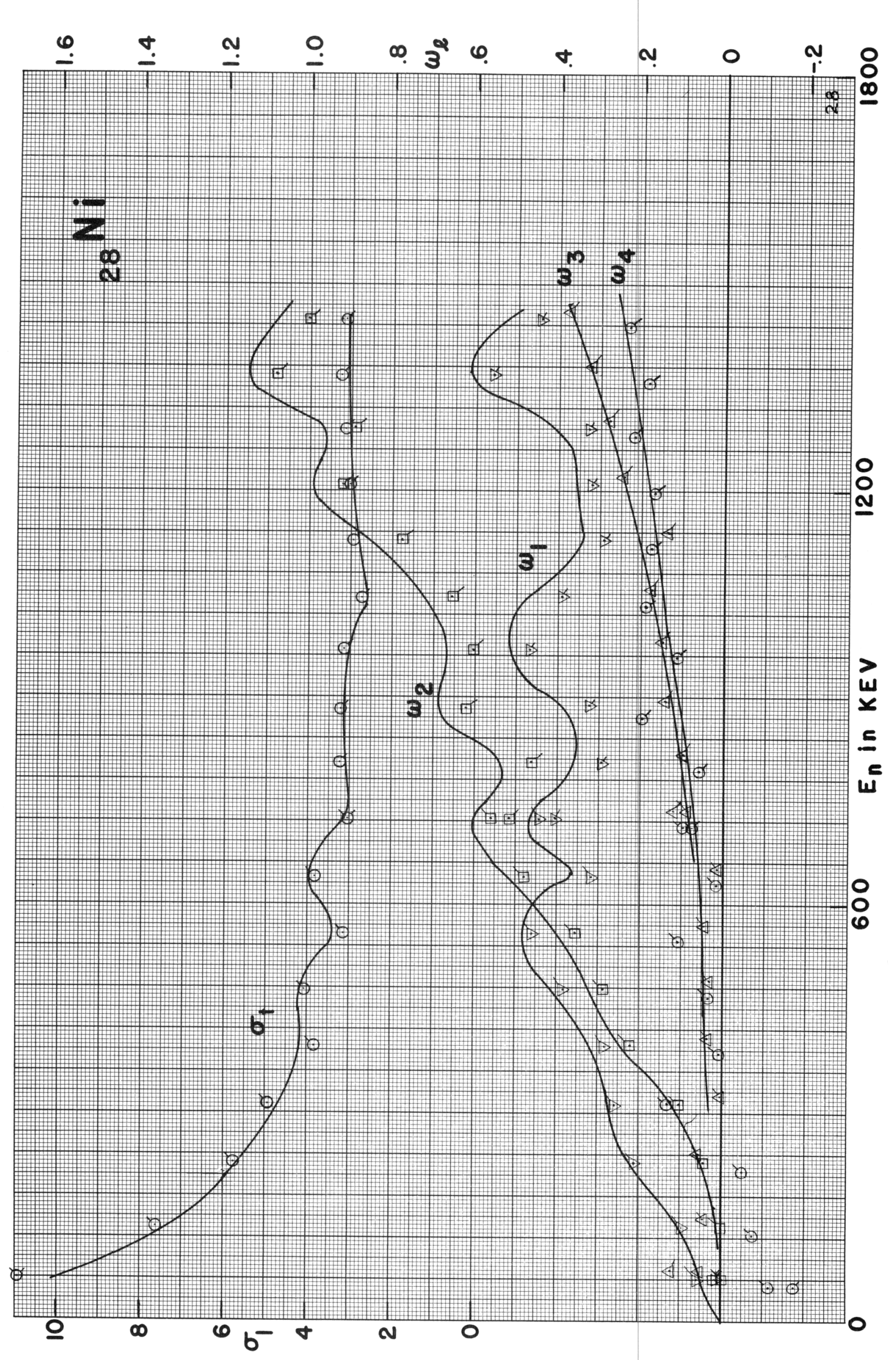




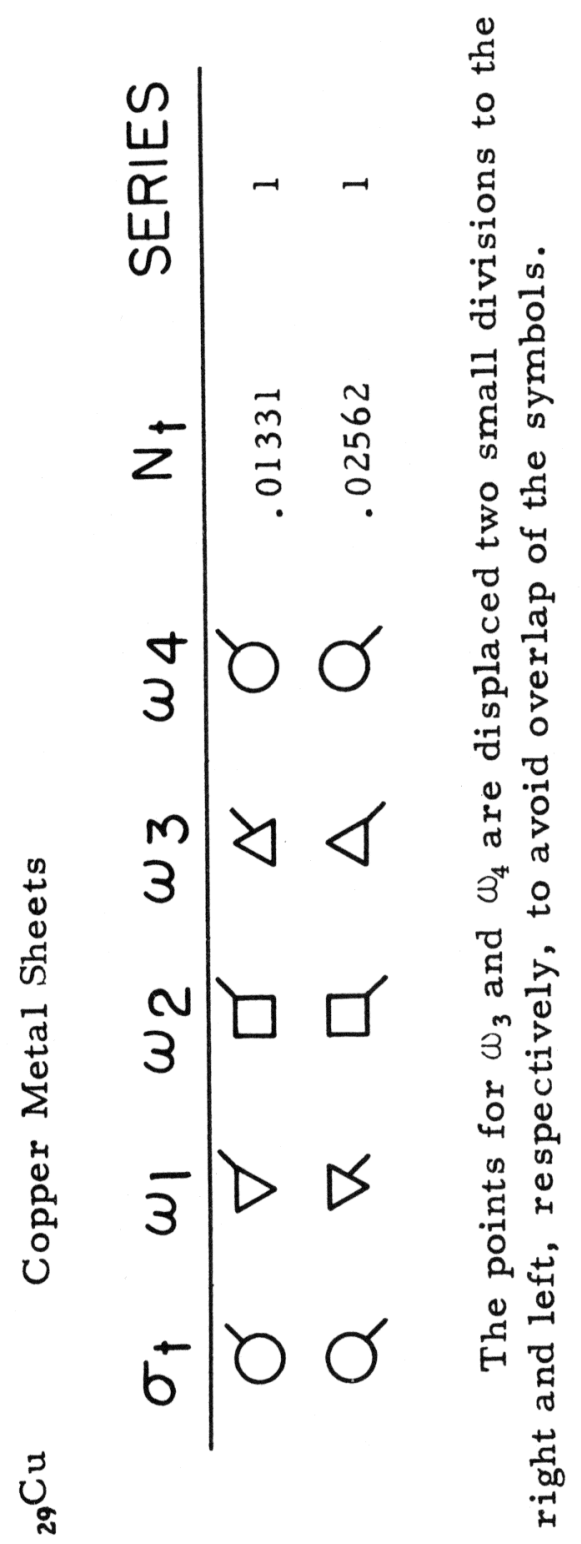




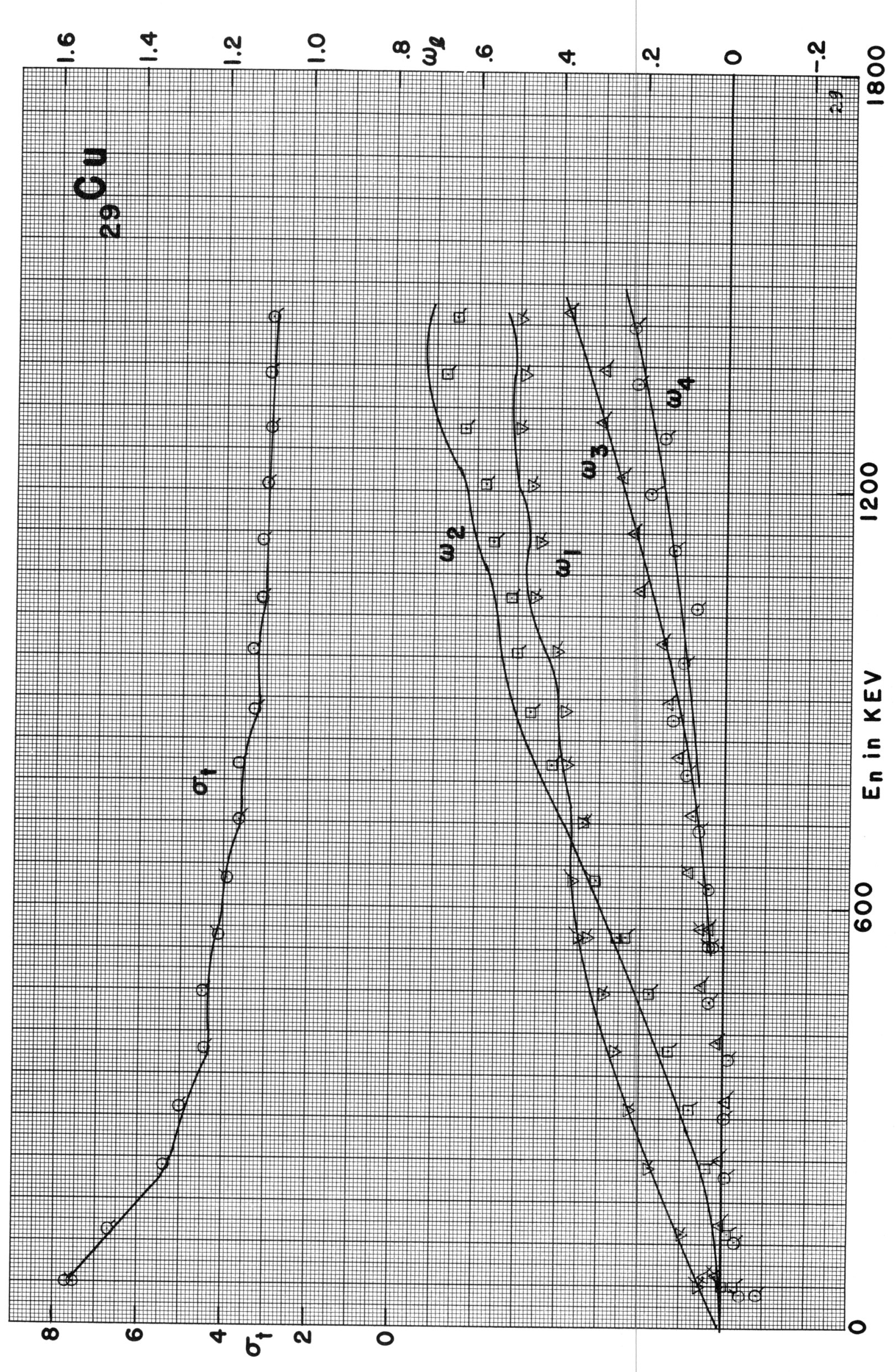




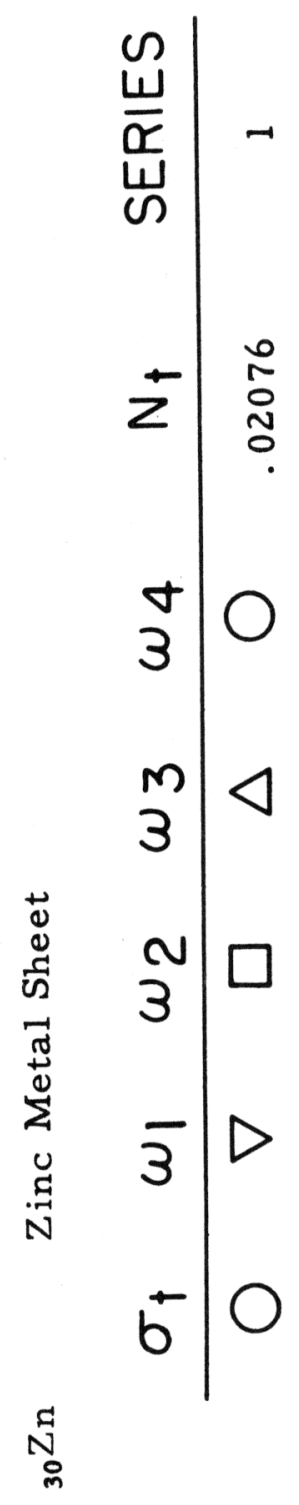




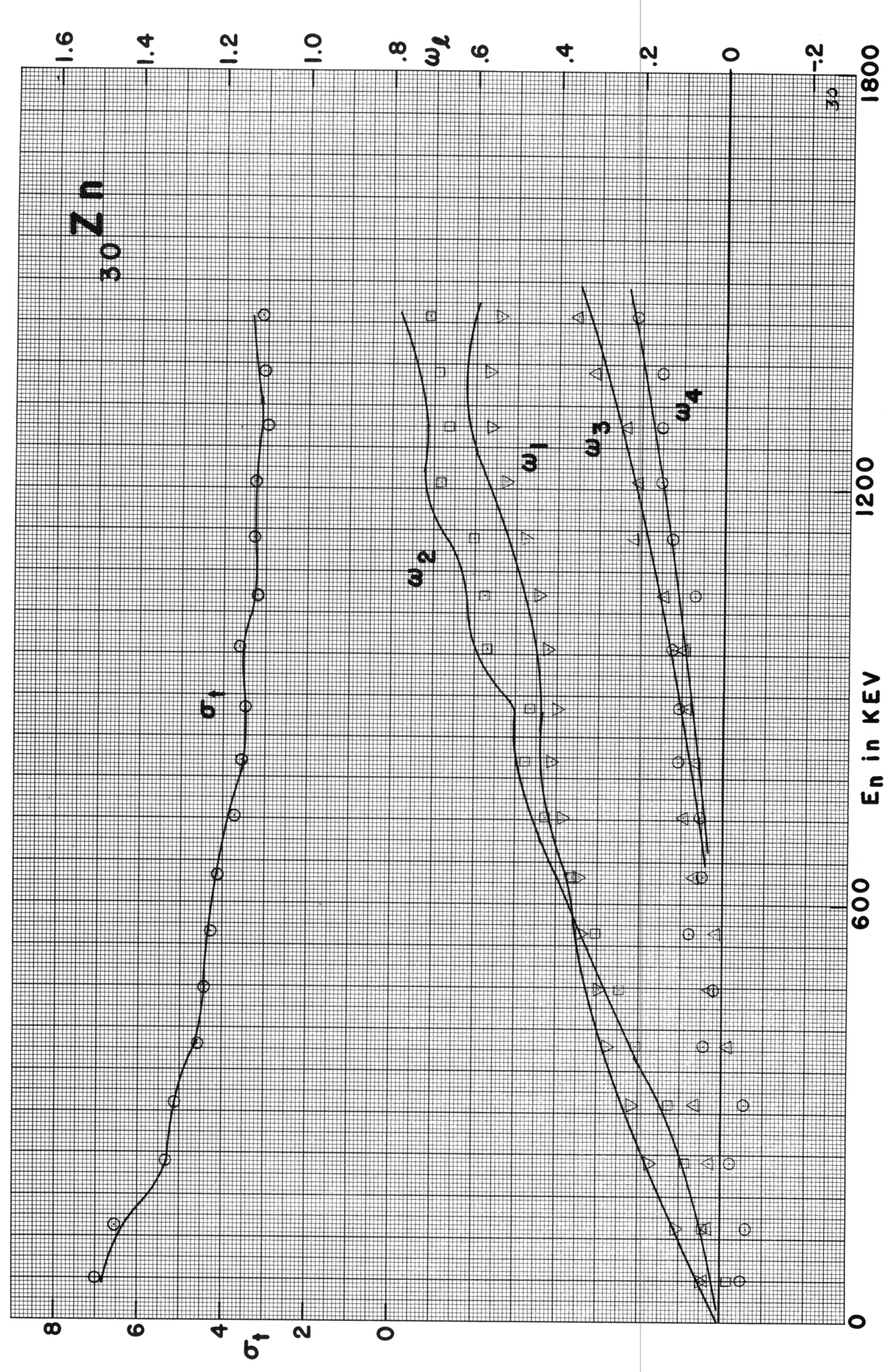




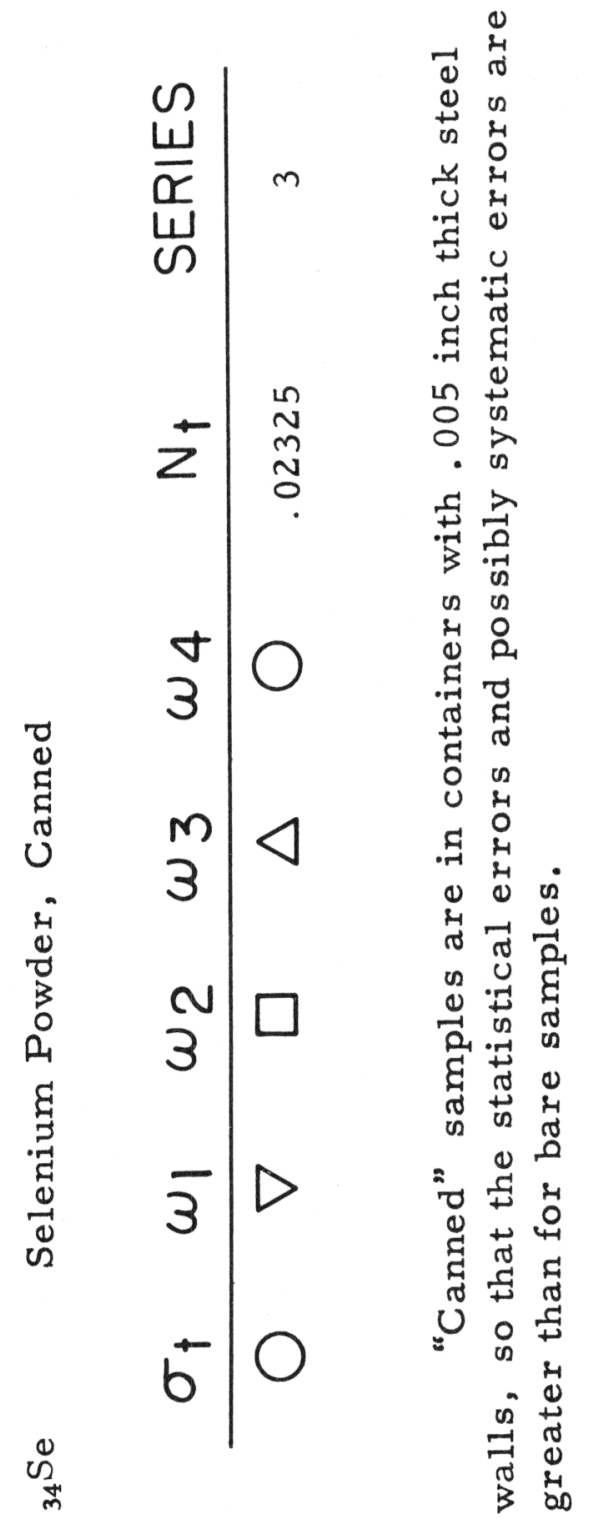




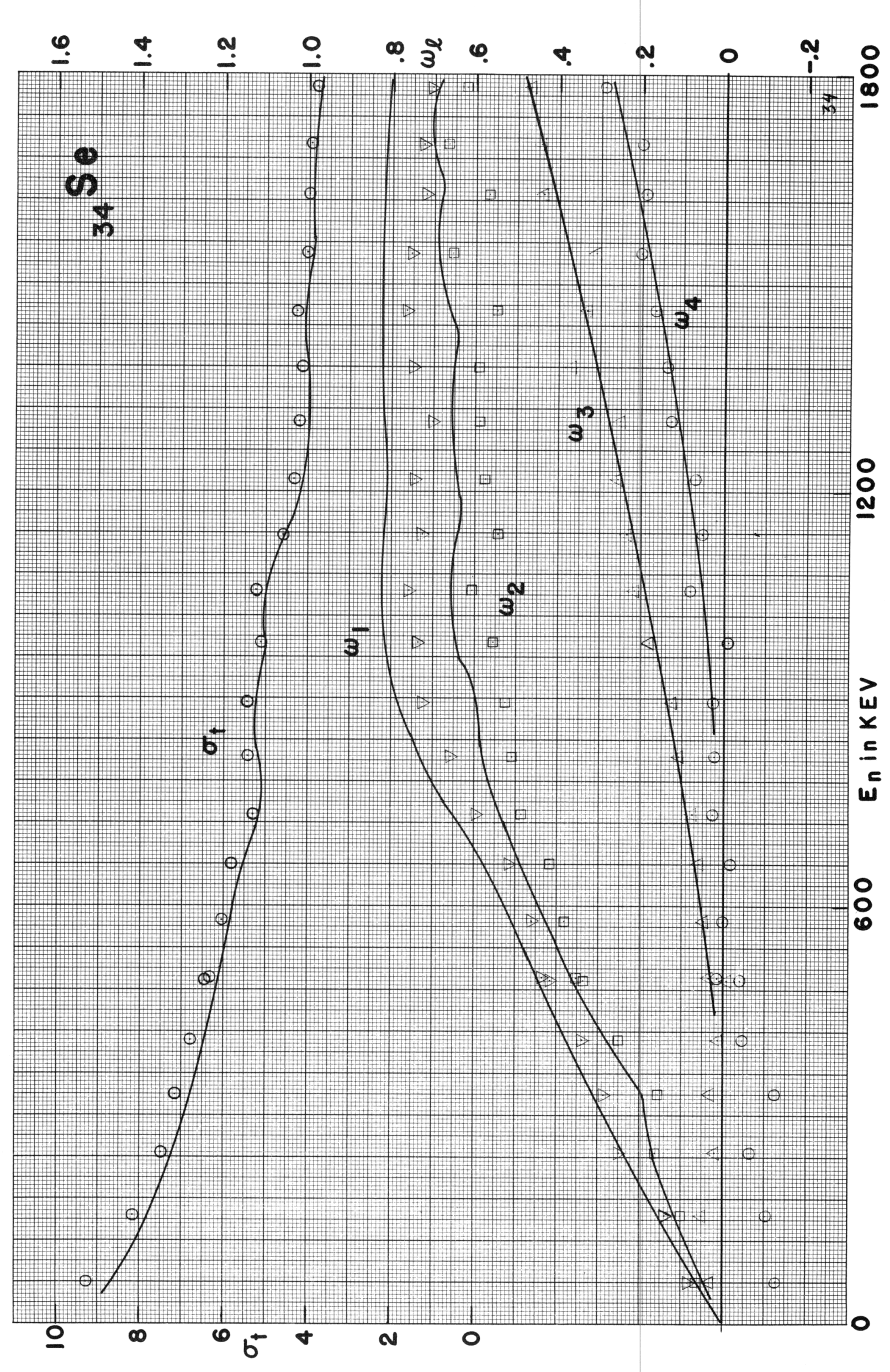




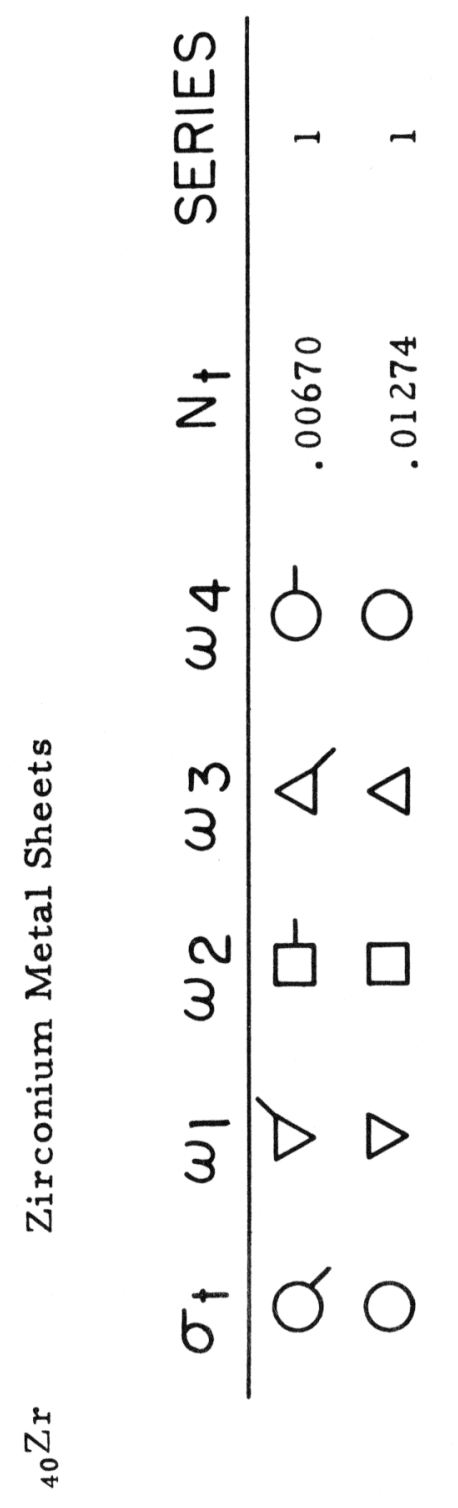




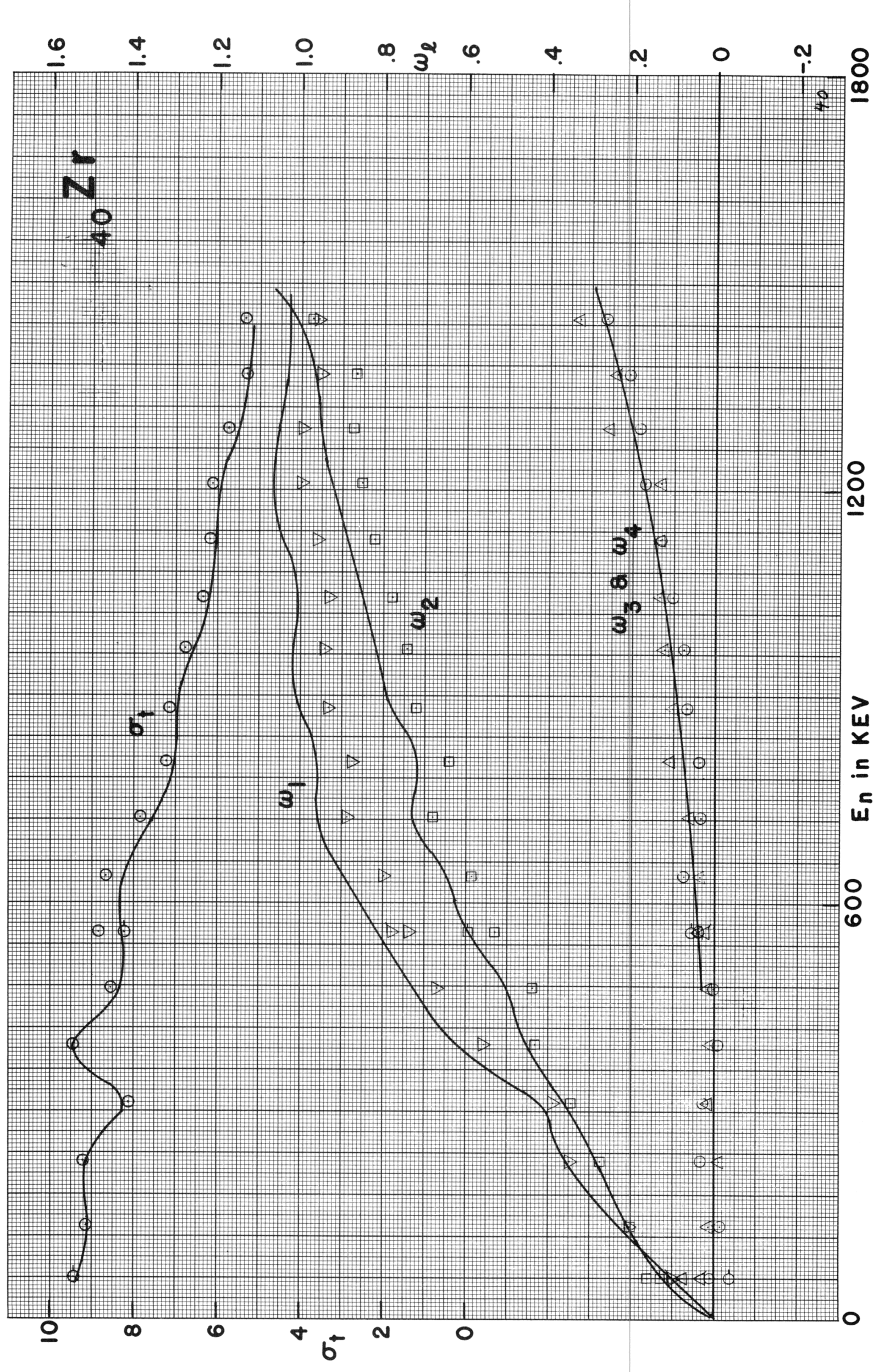




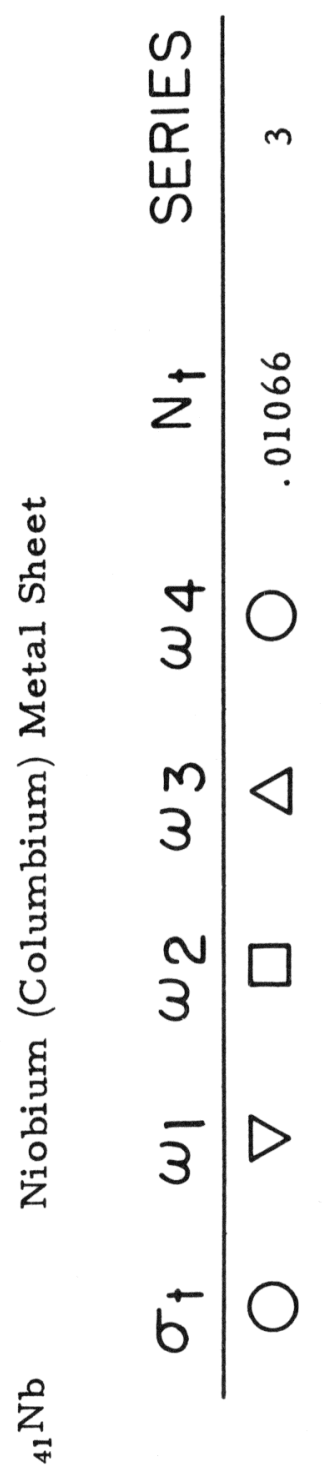




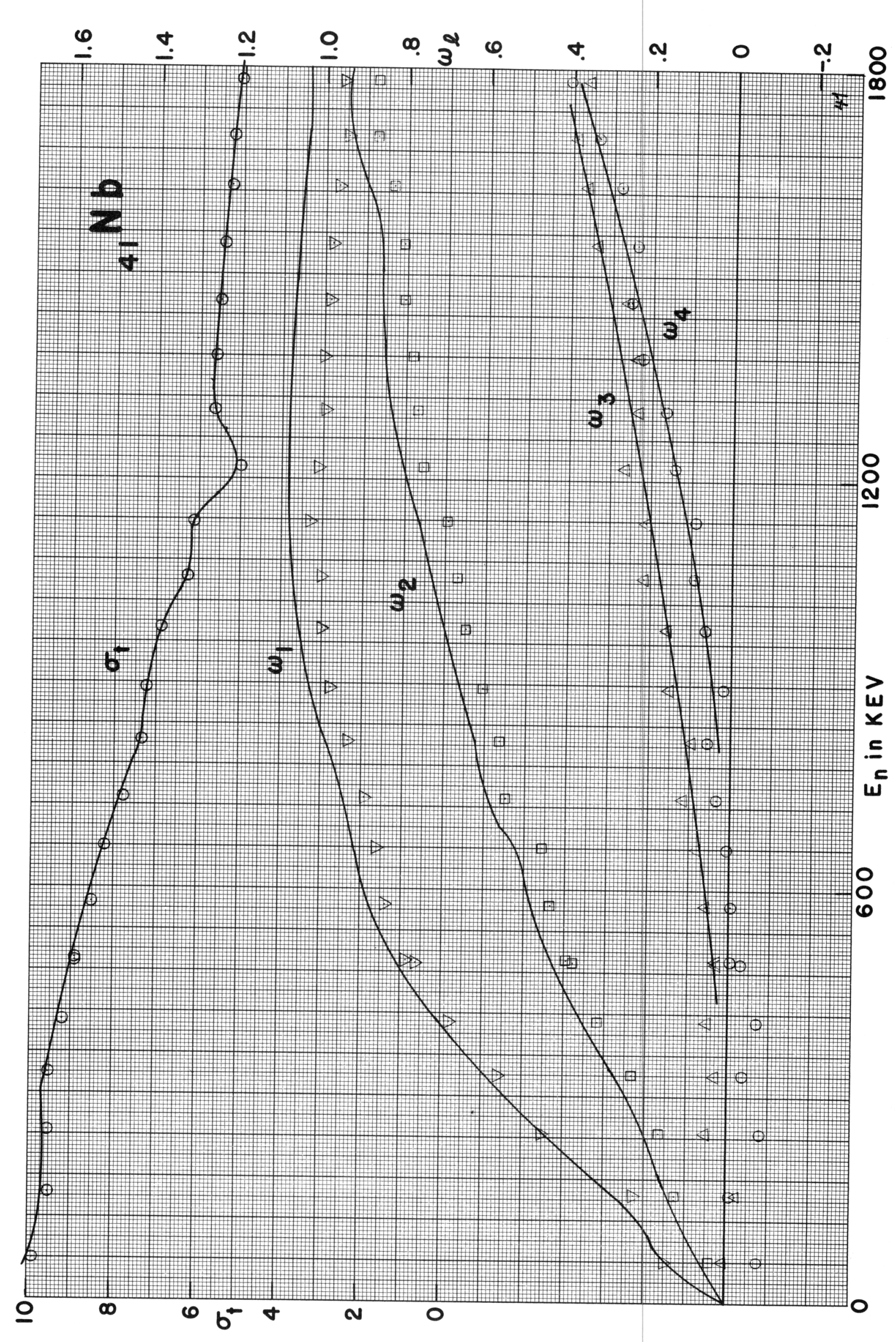




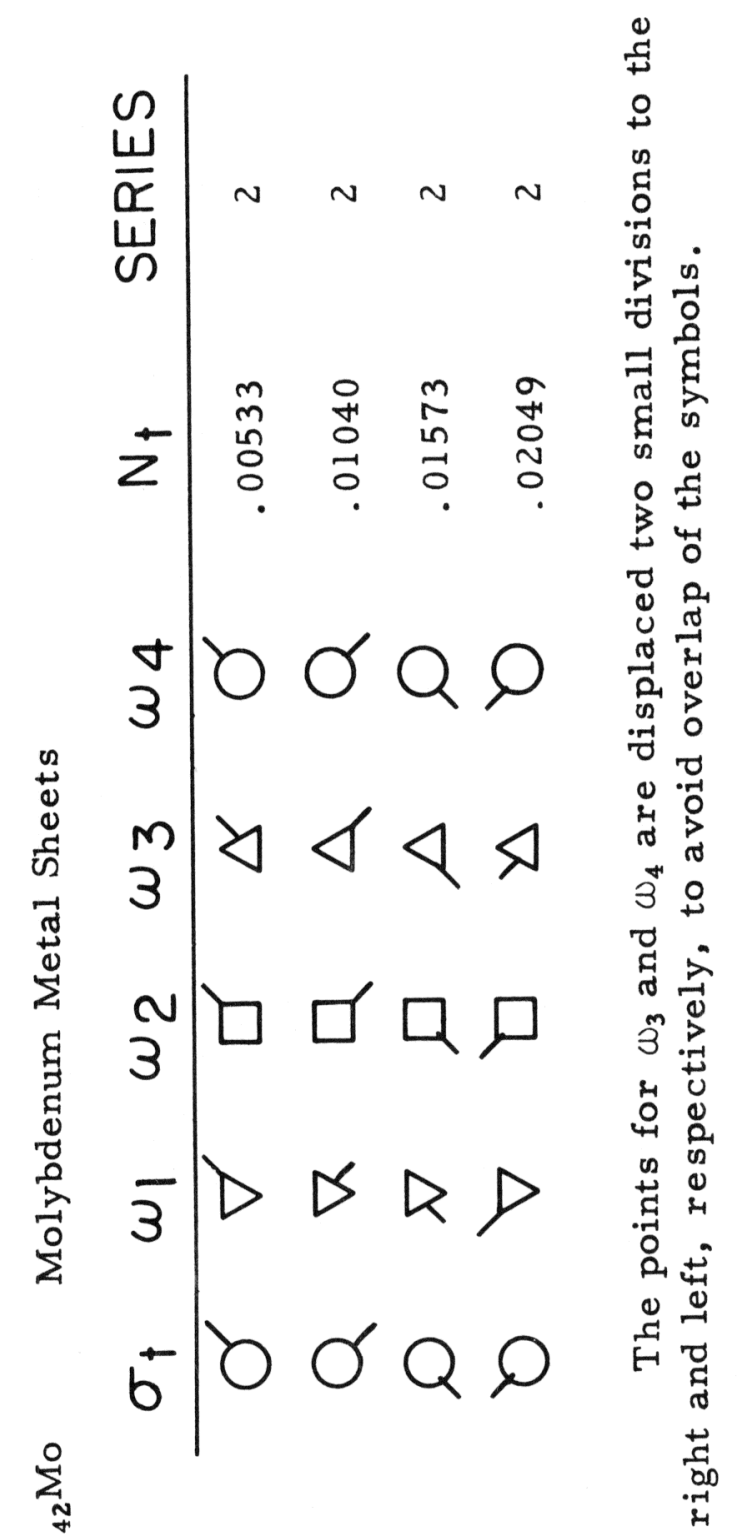




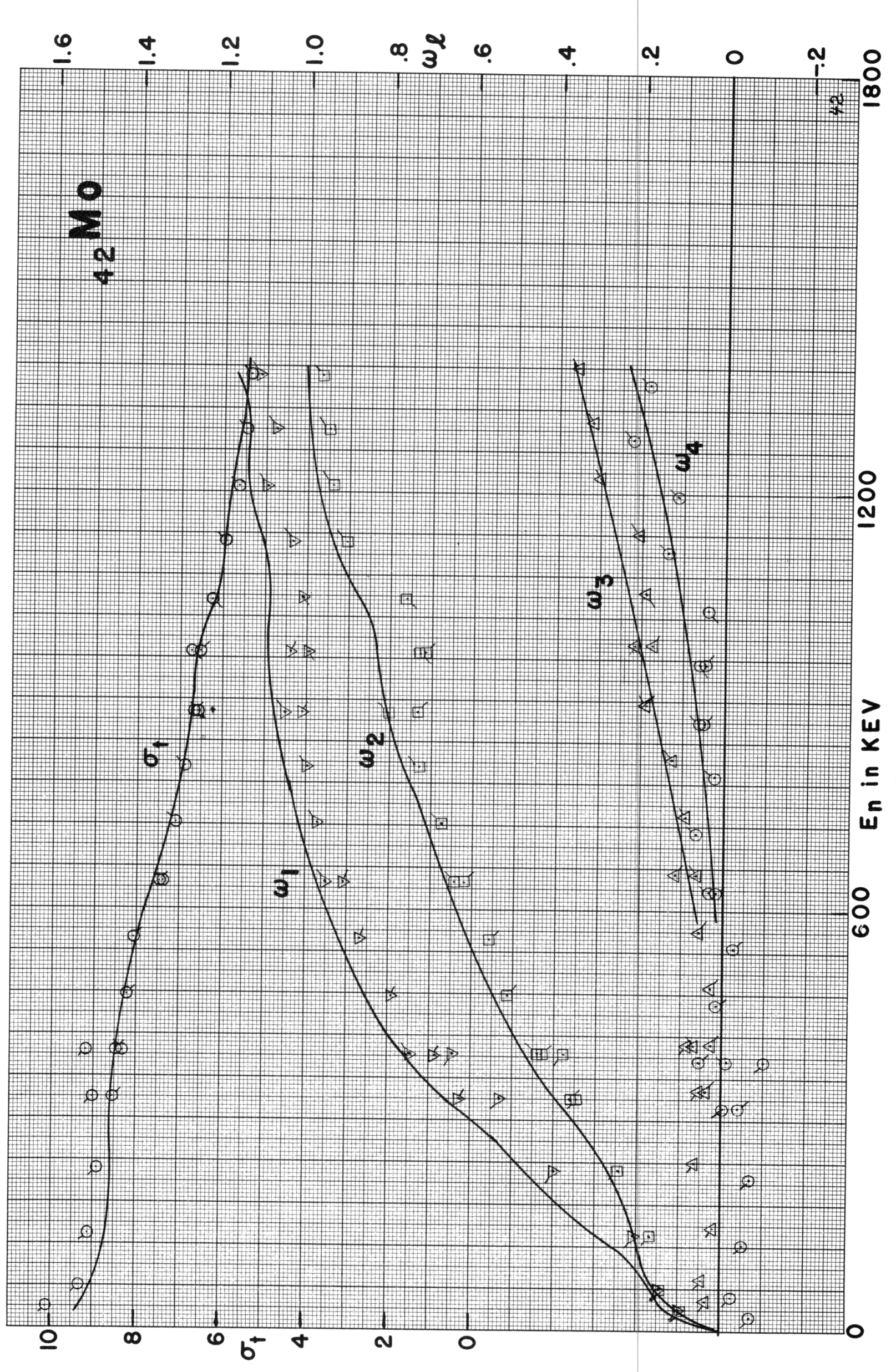




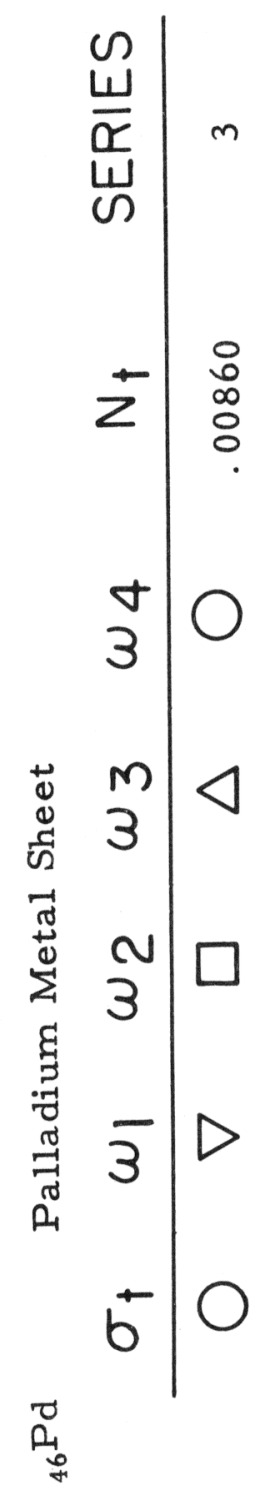




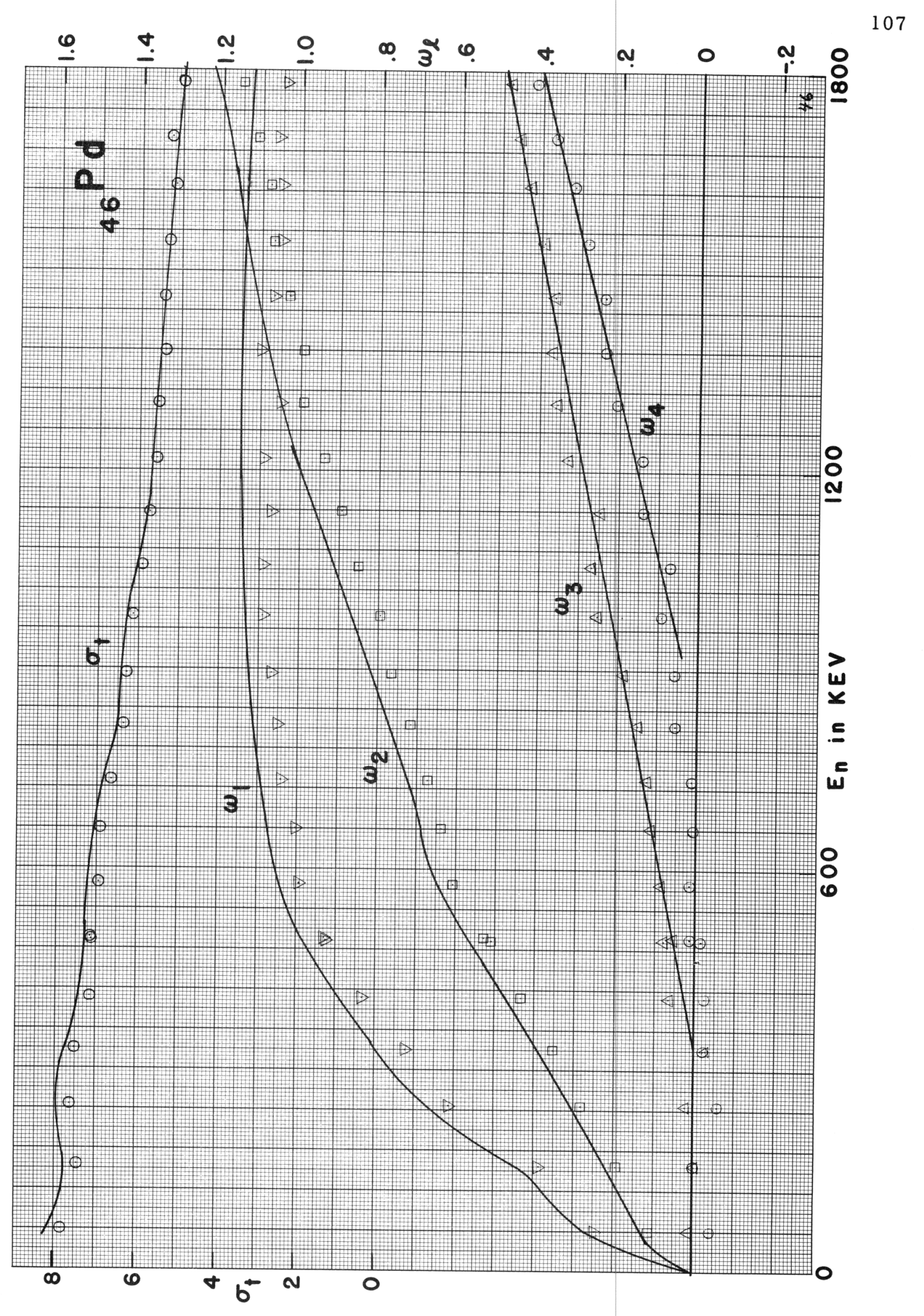




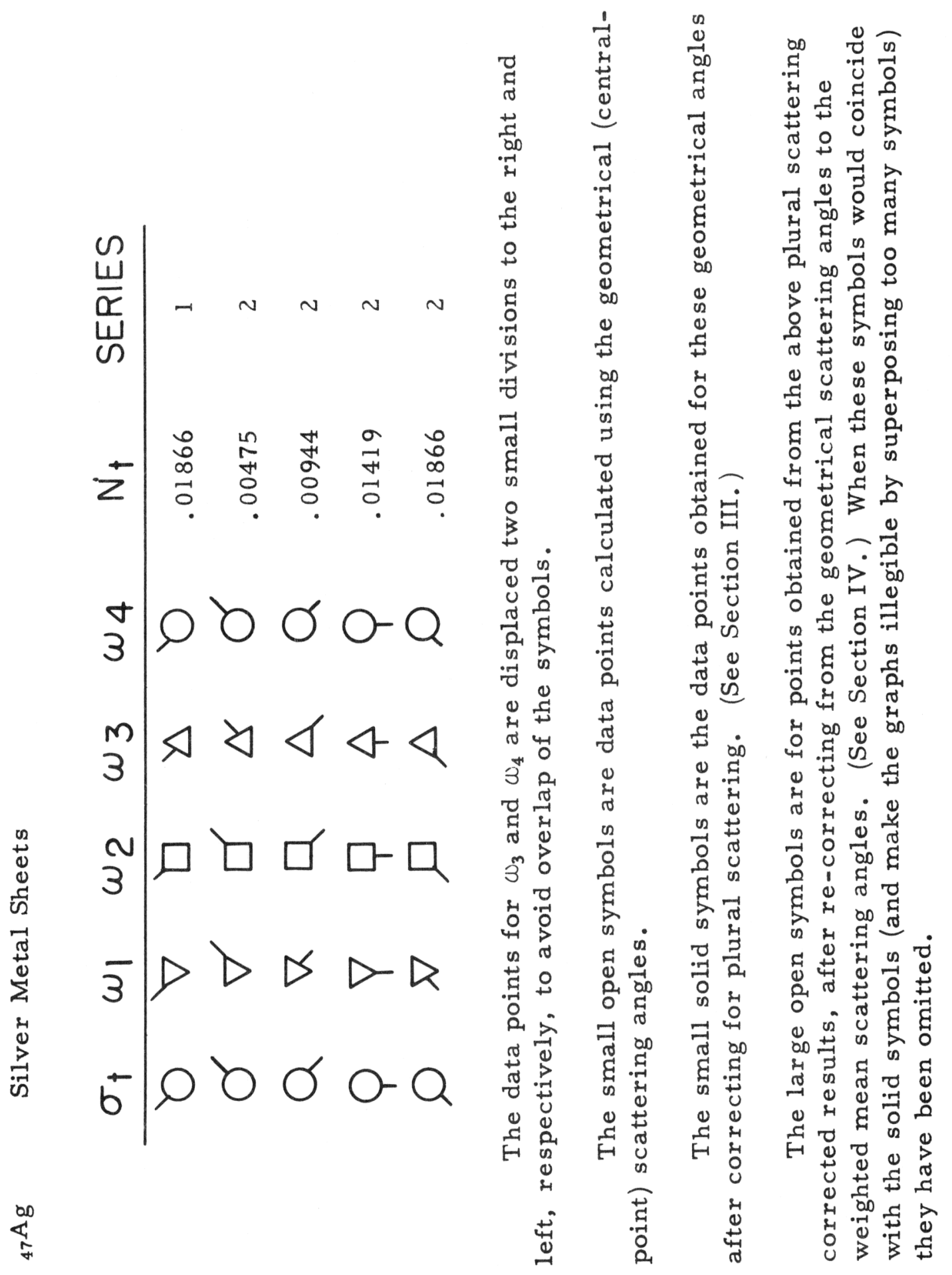




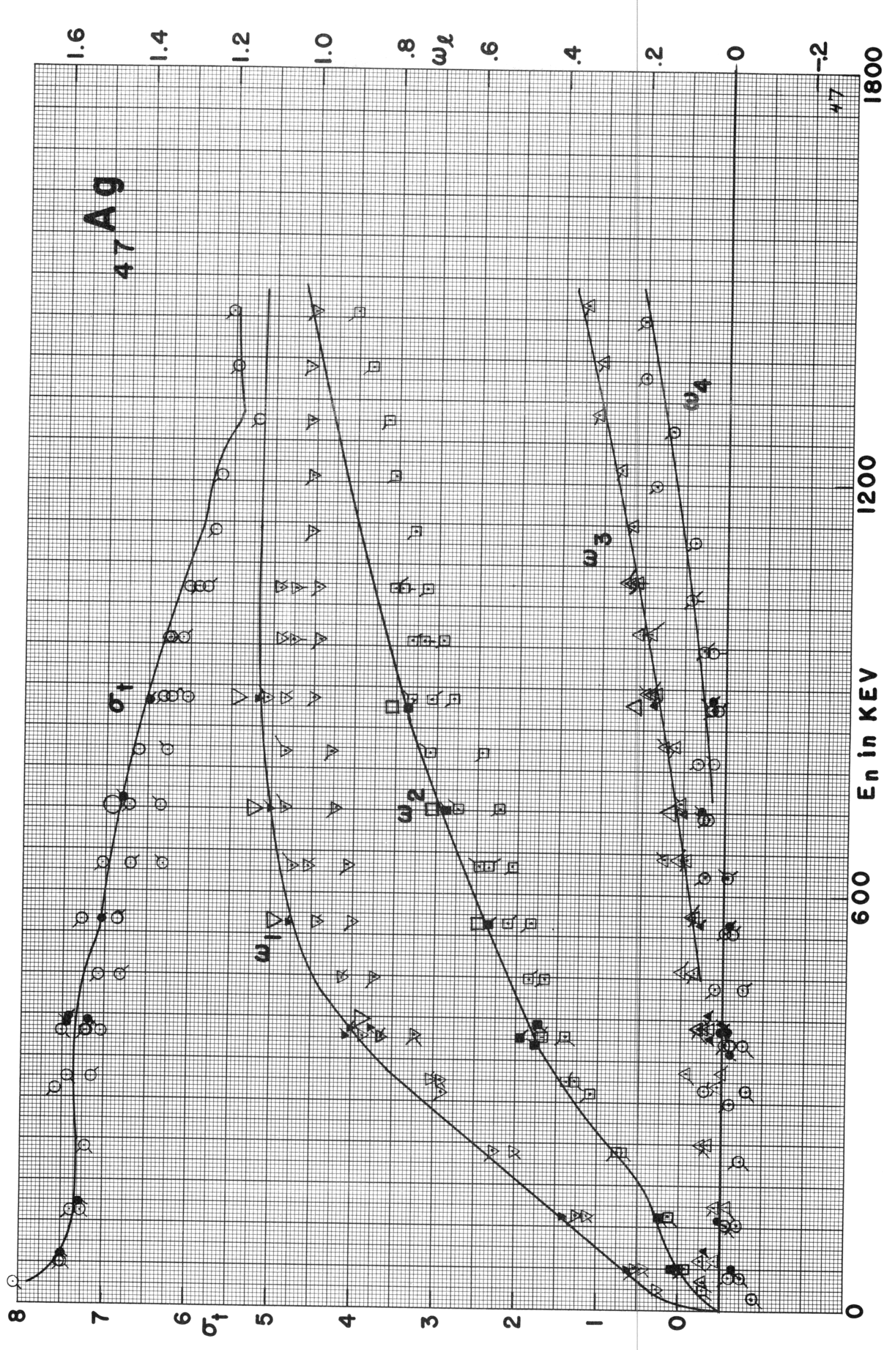




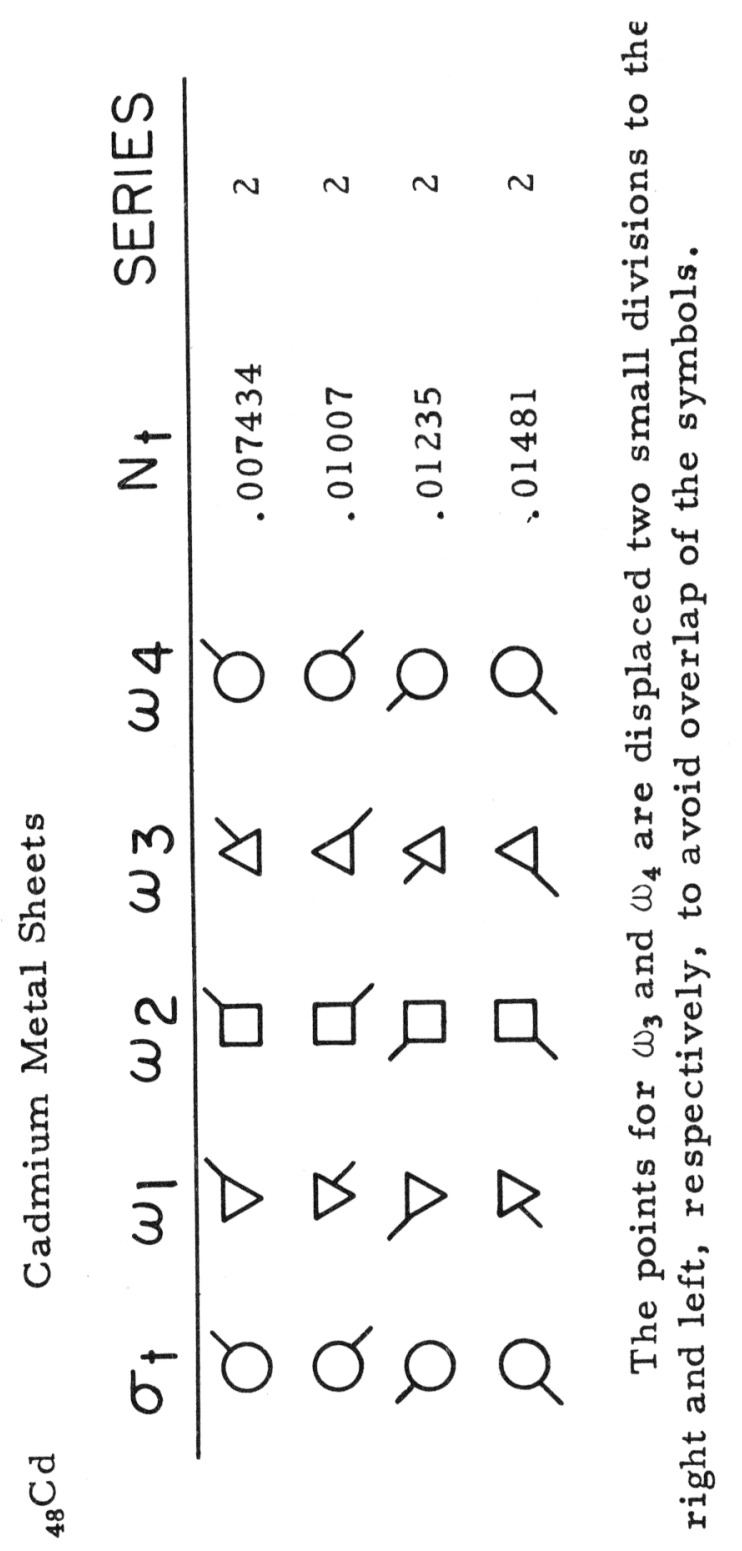




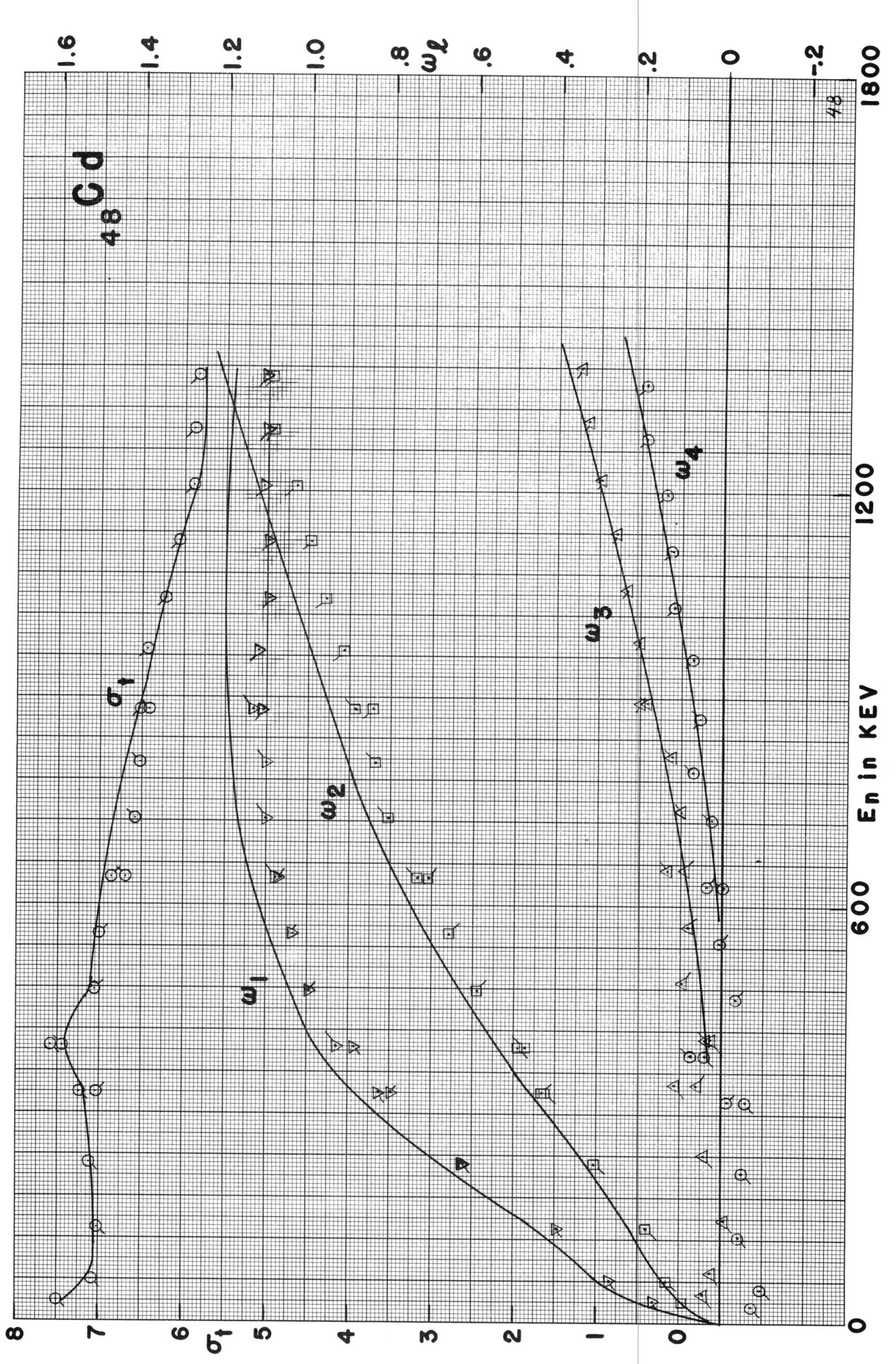




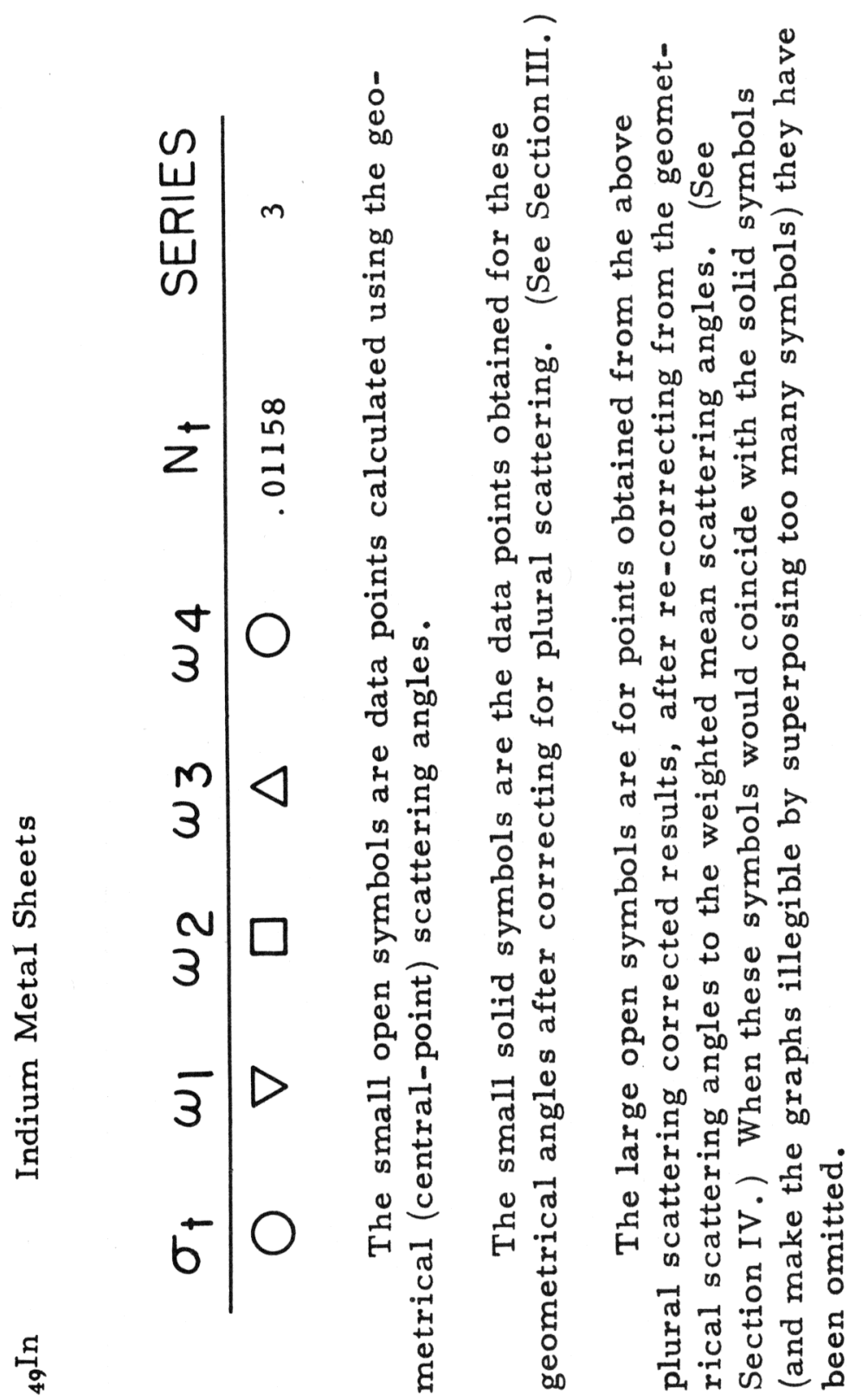




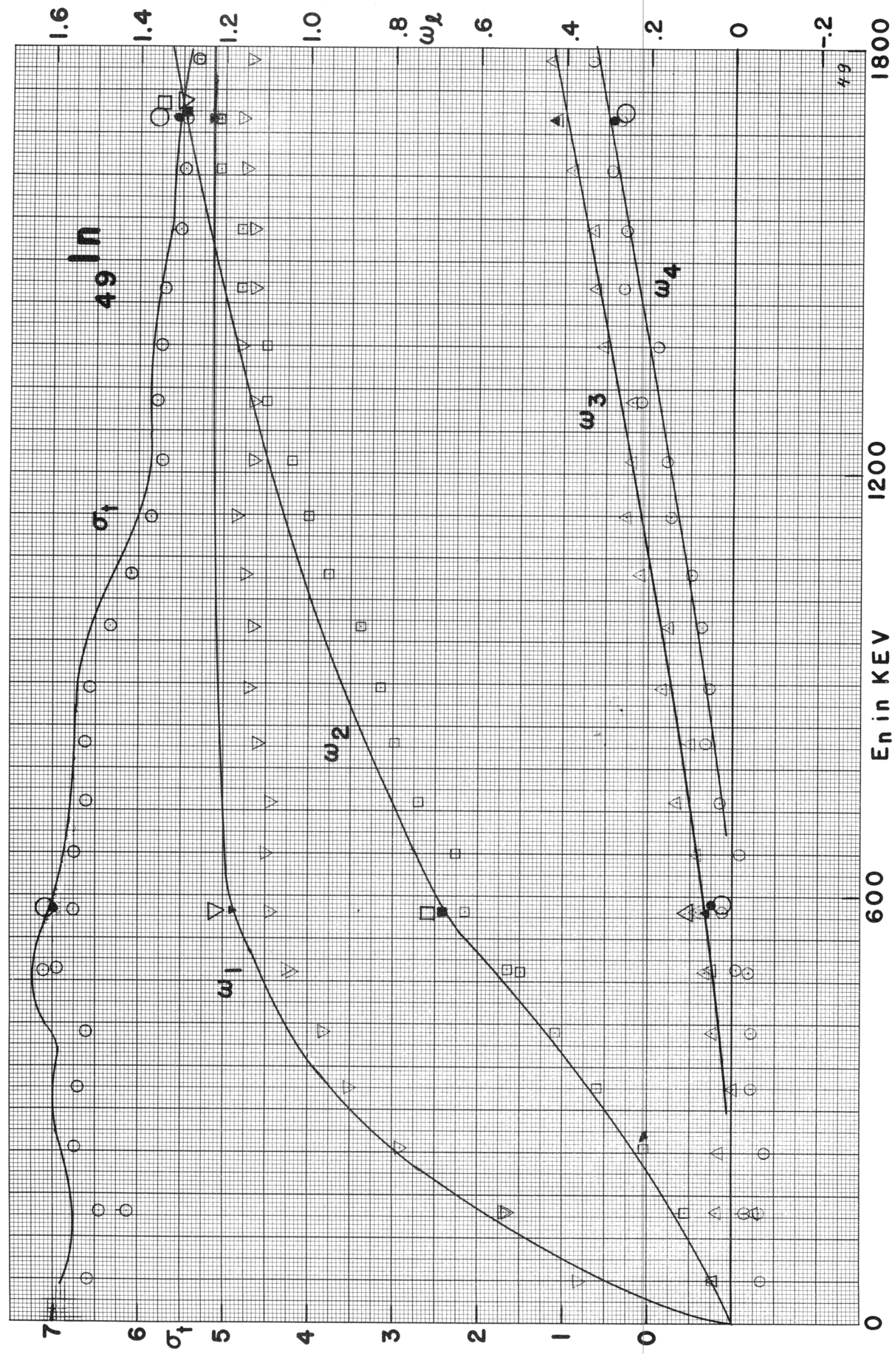




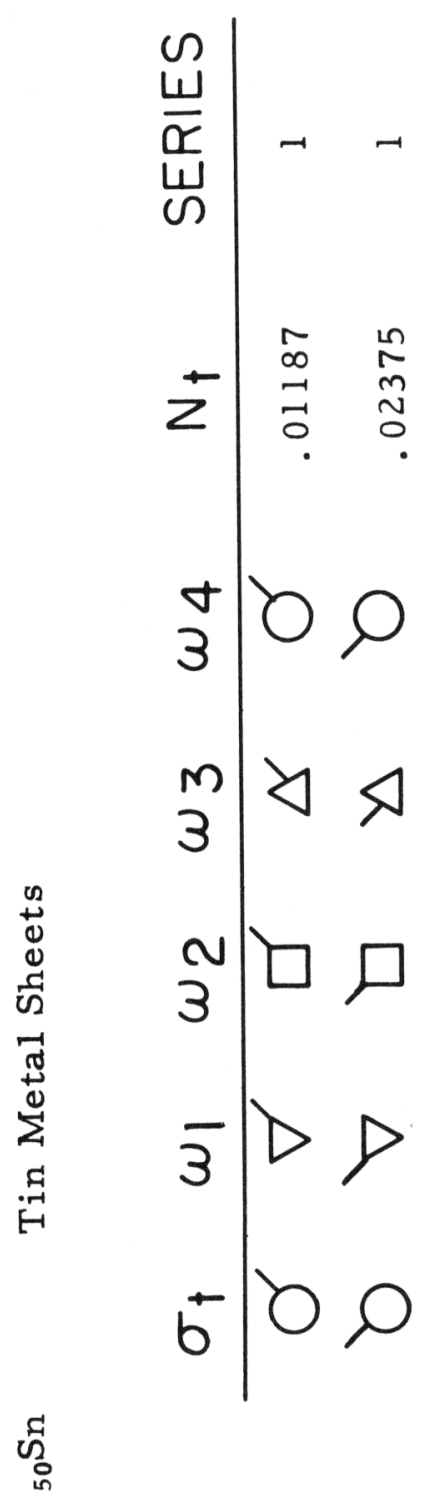




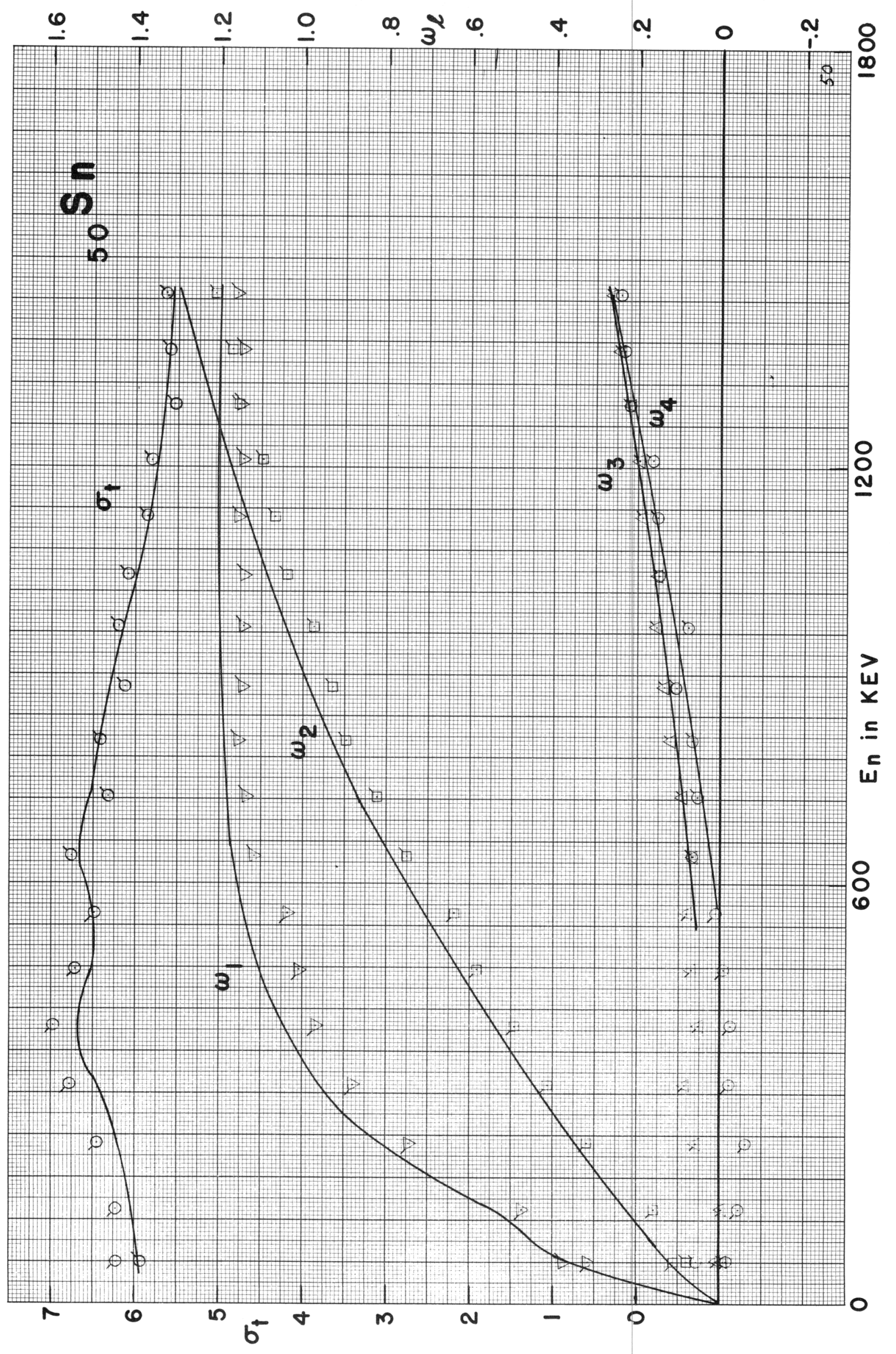




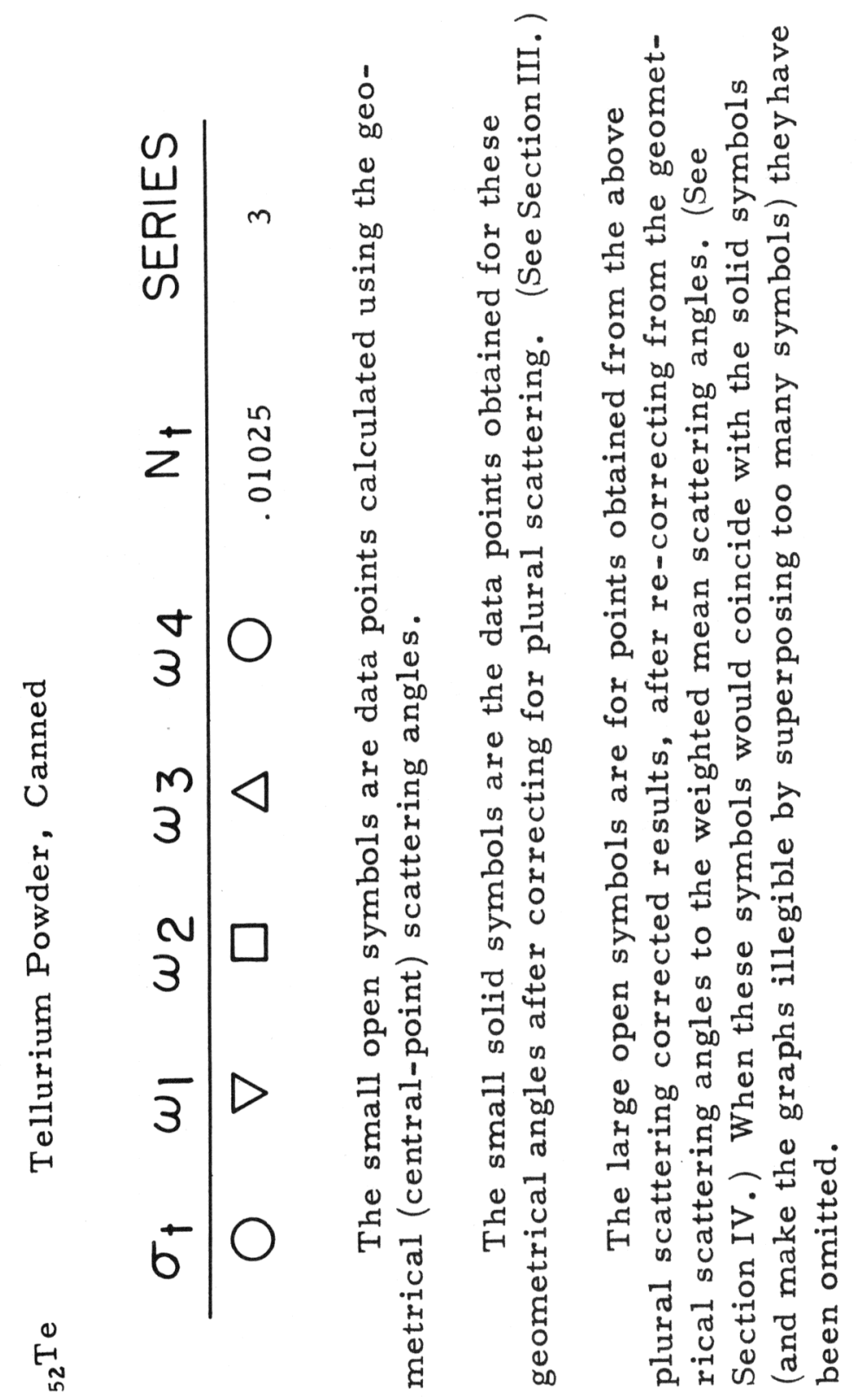




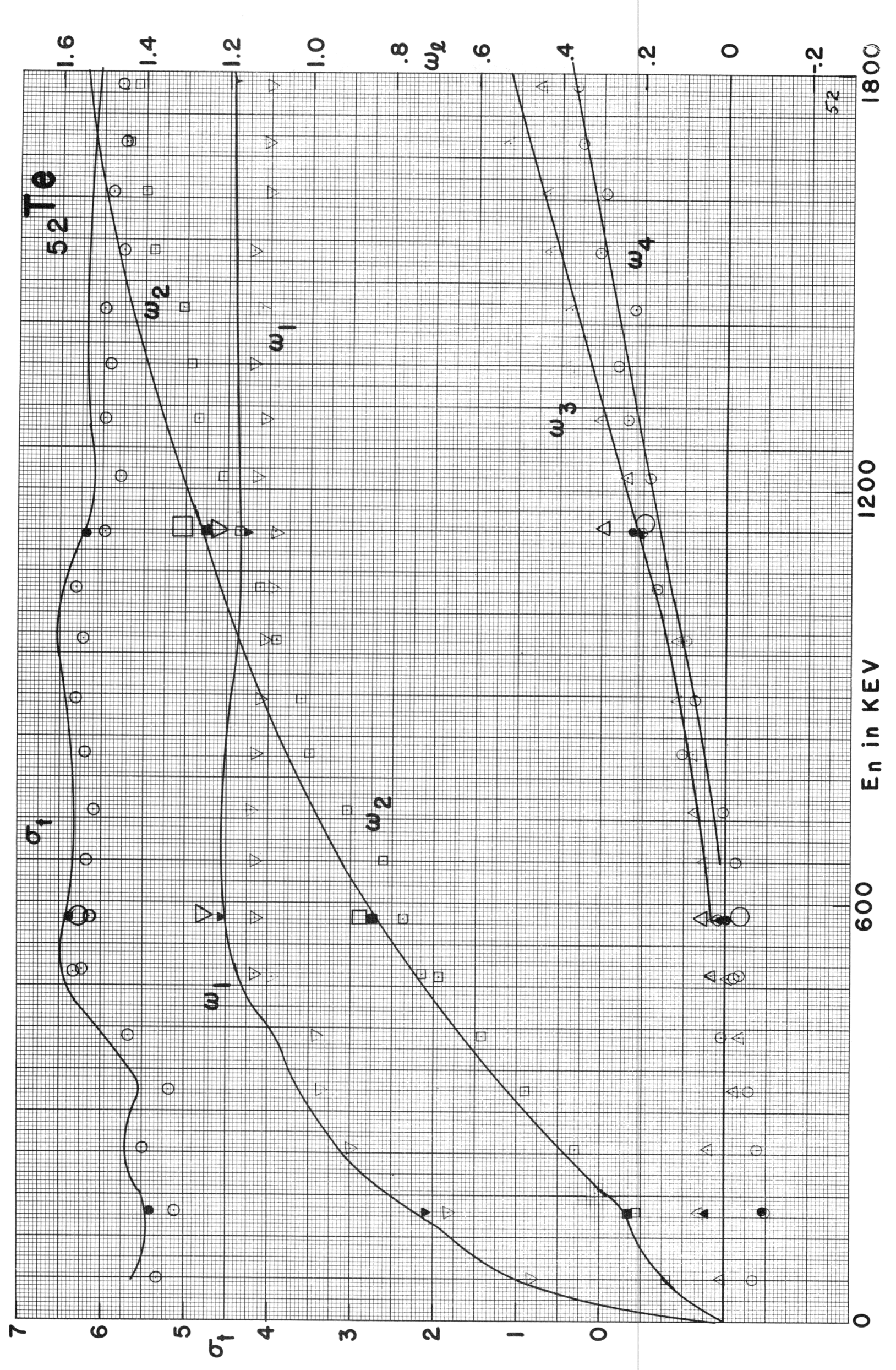




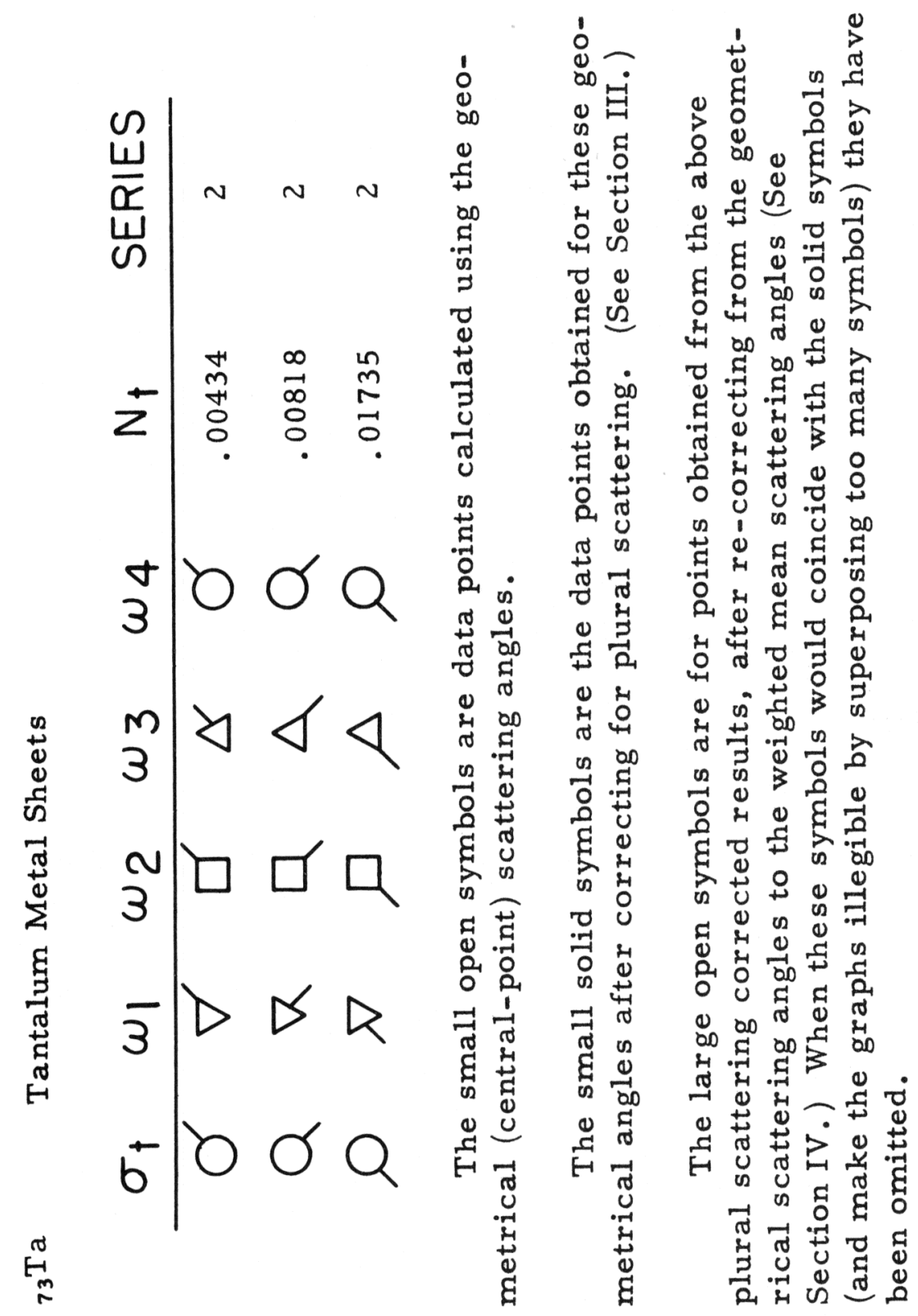




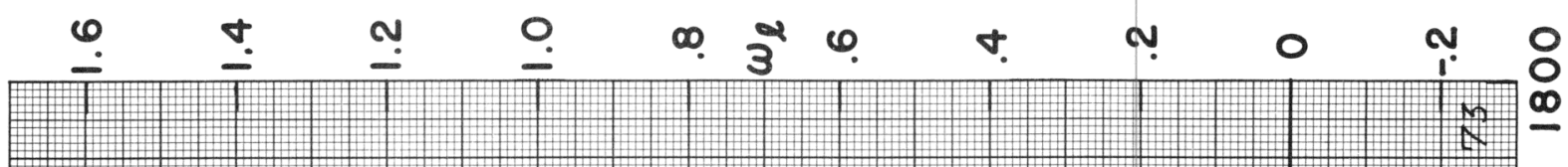
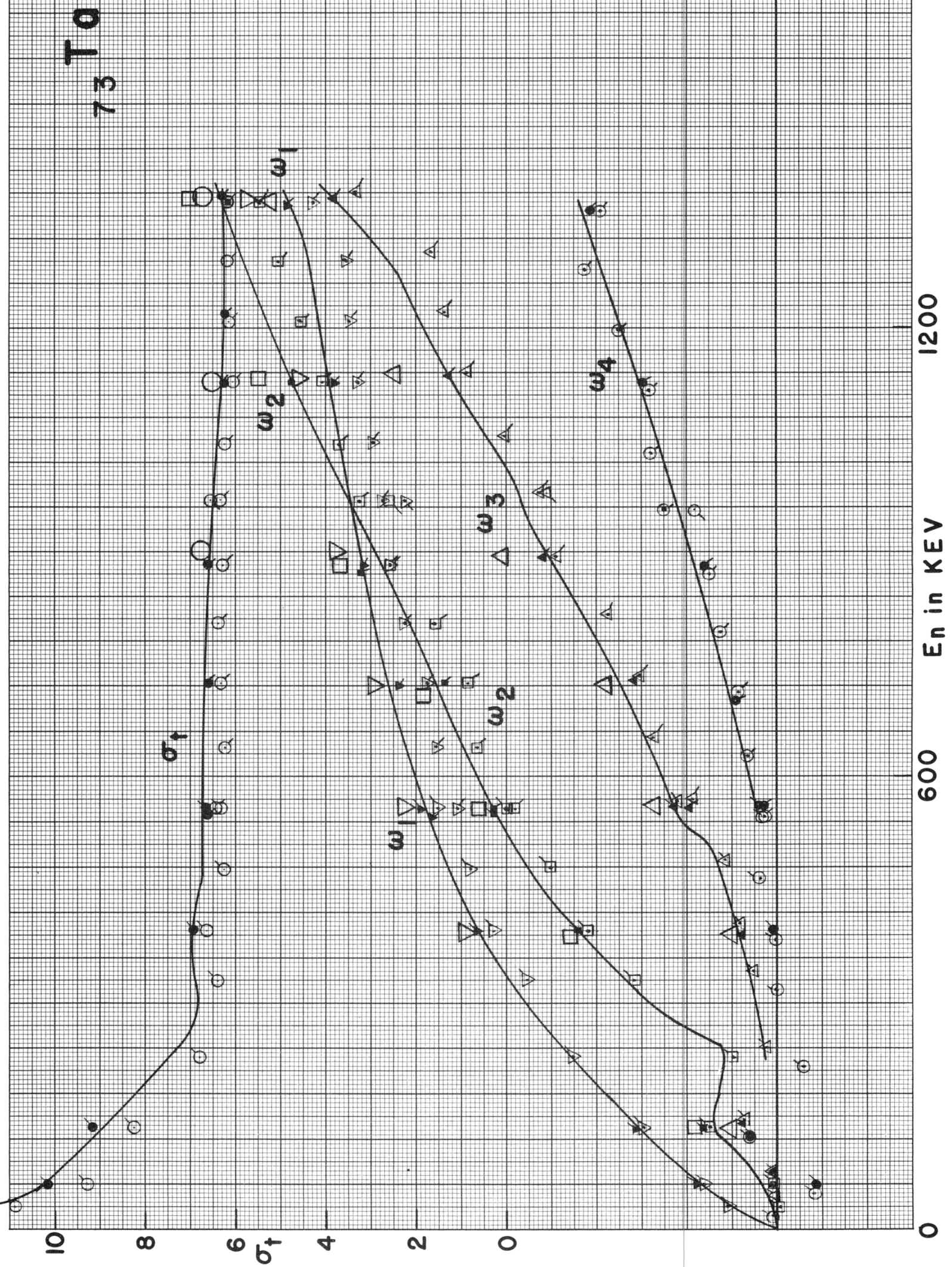


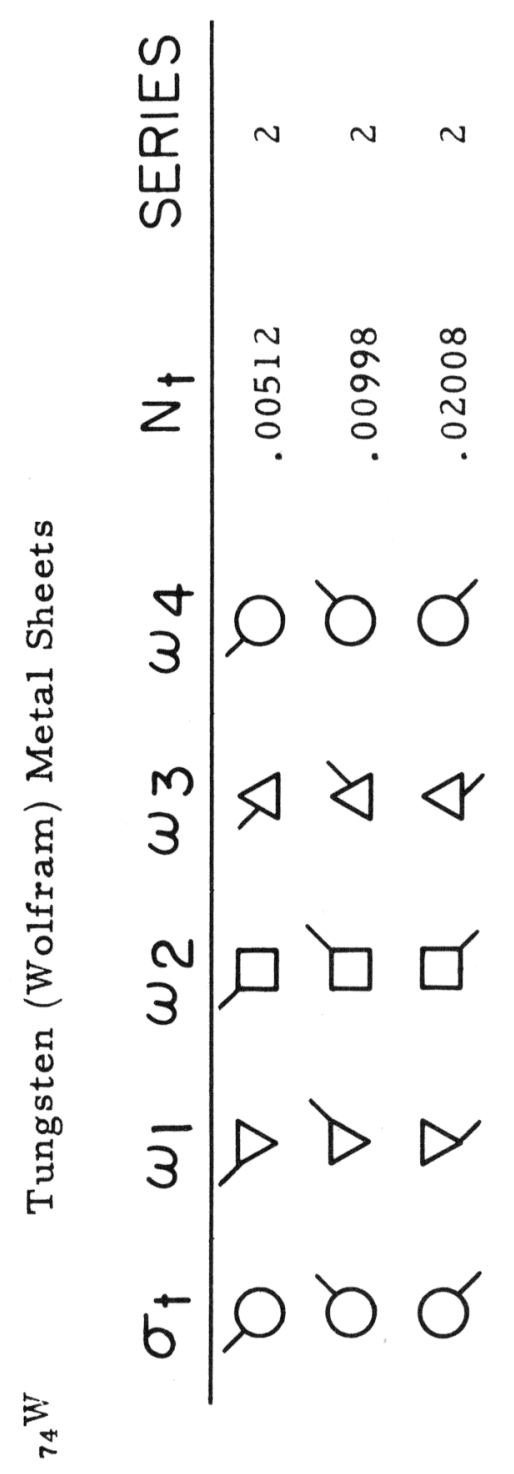




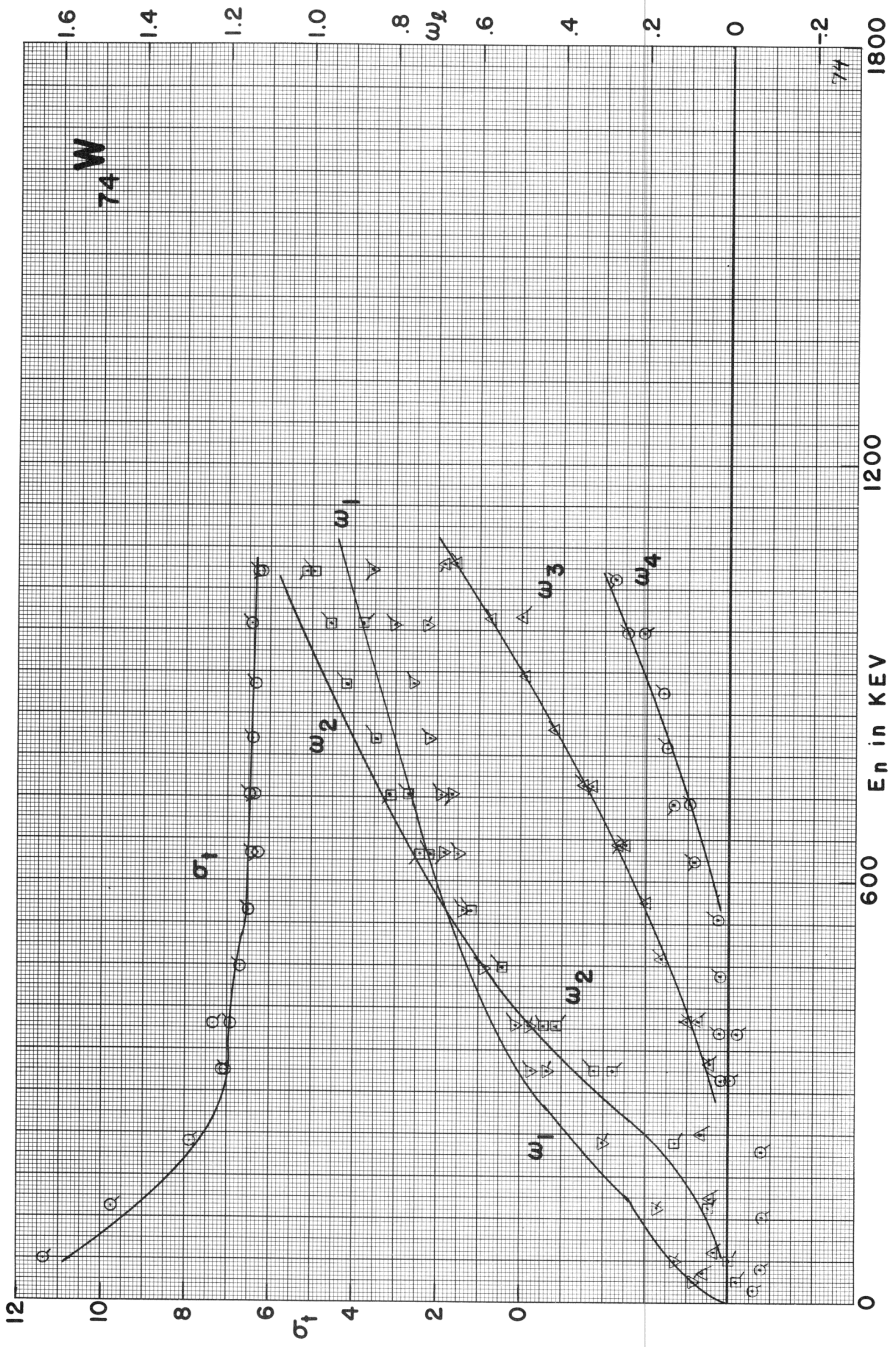


122

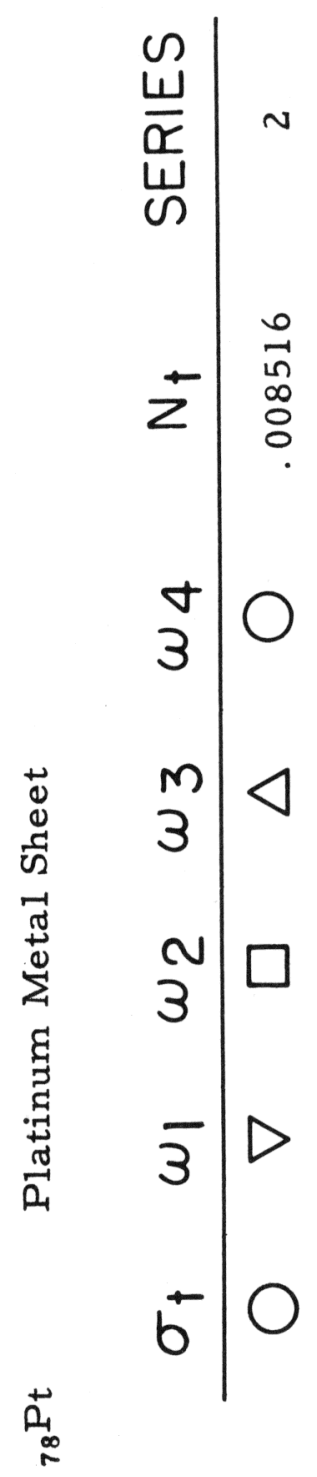




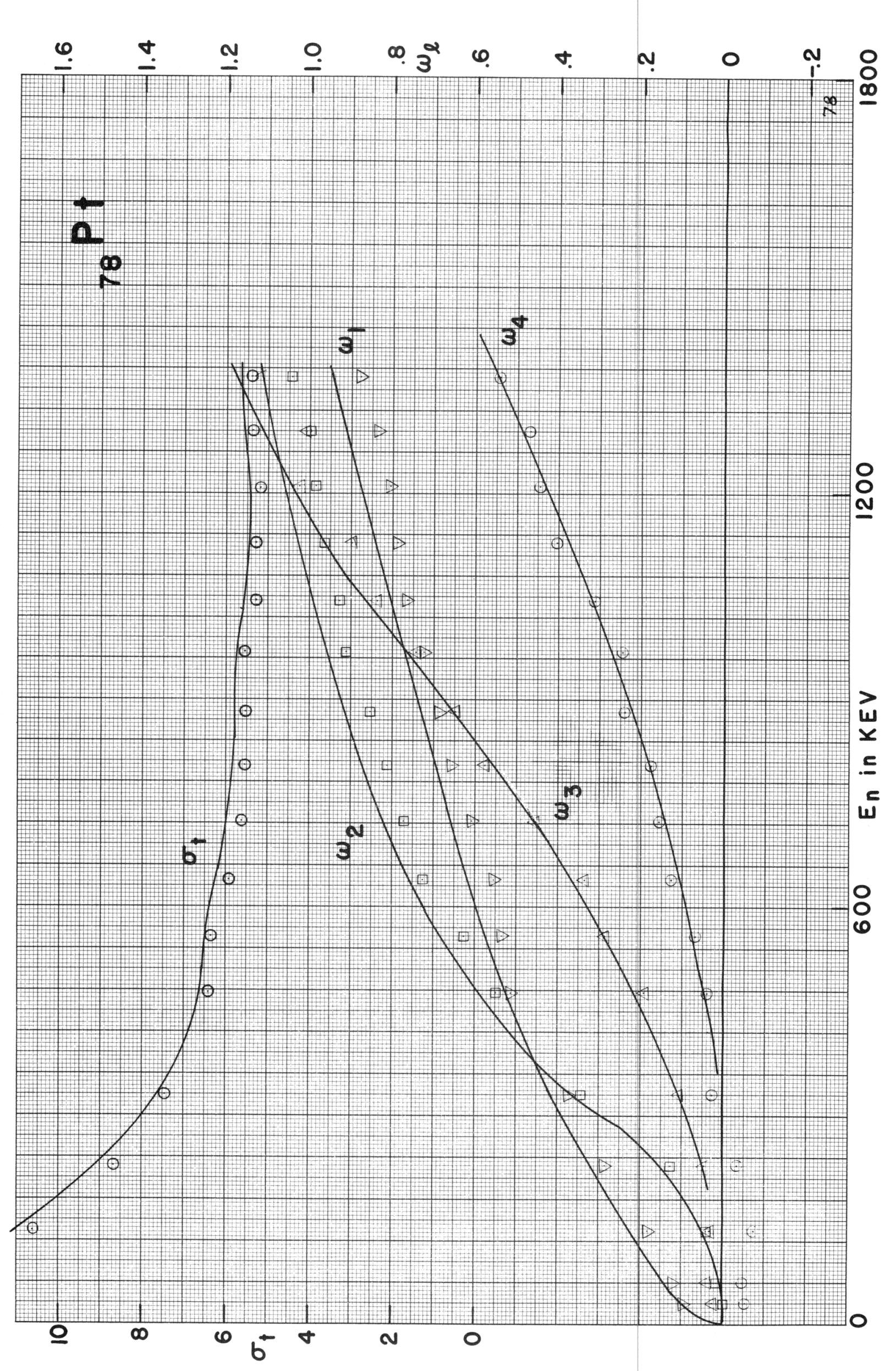




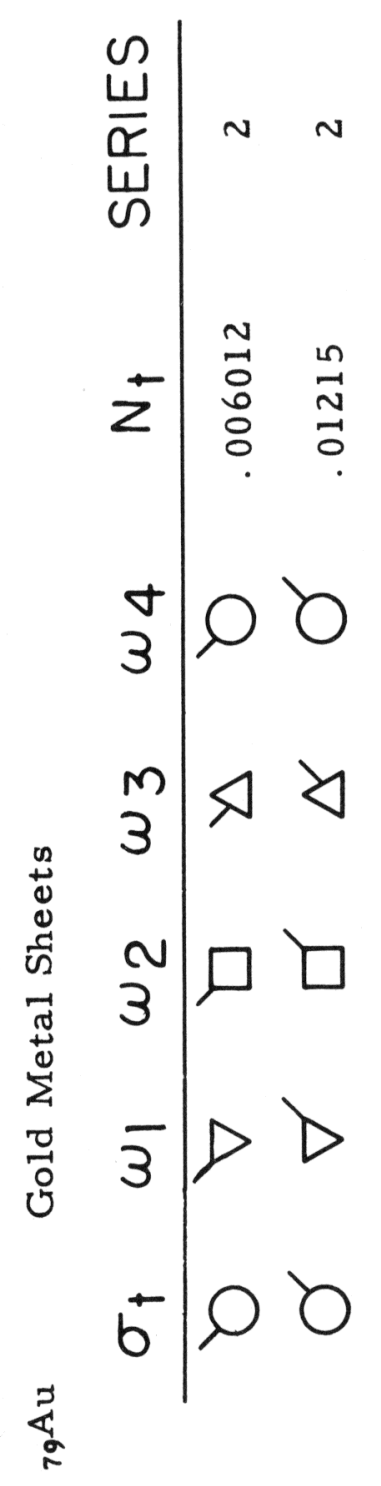




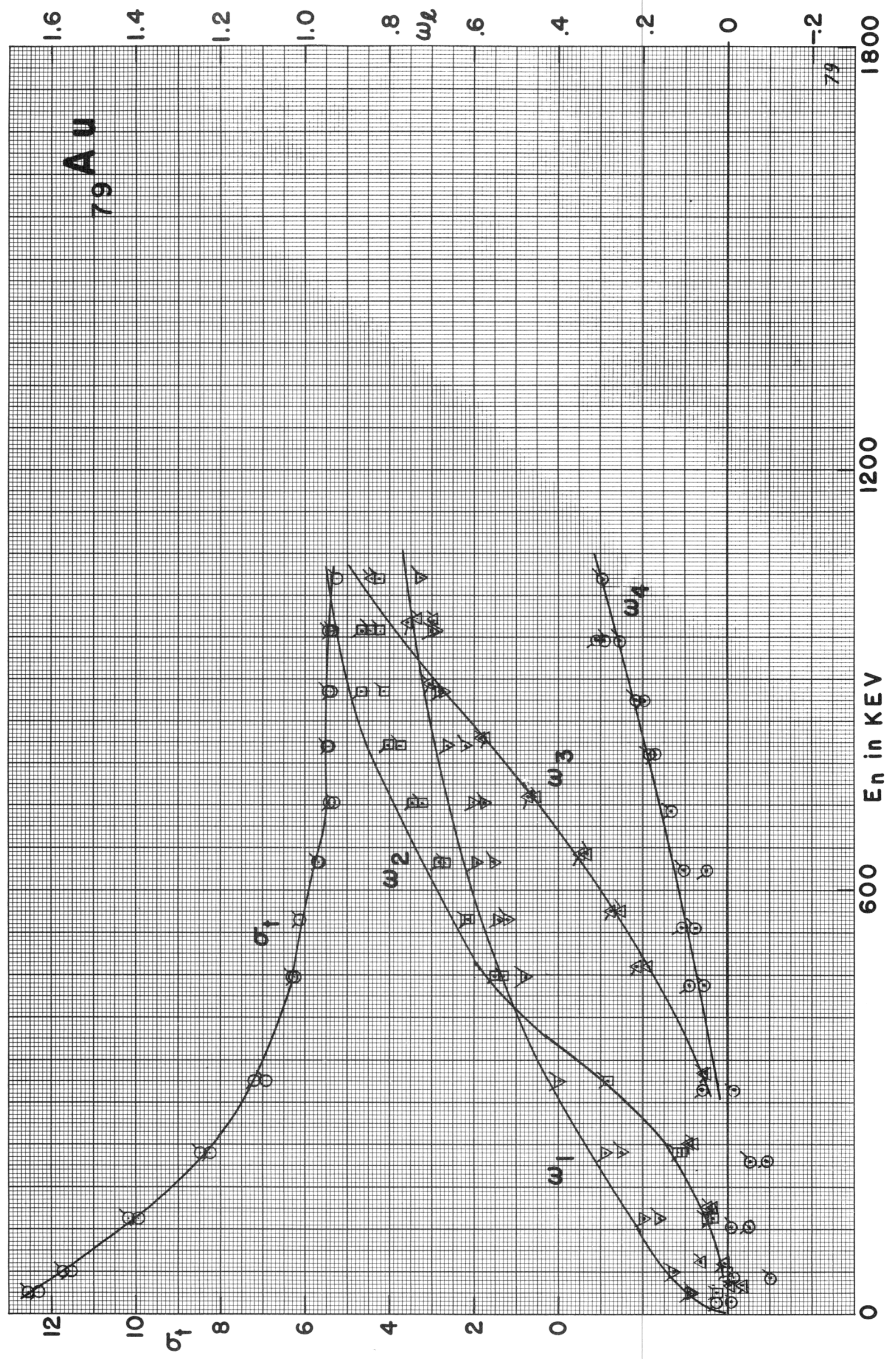




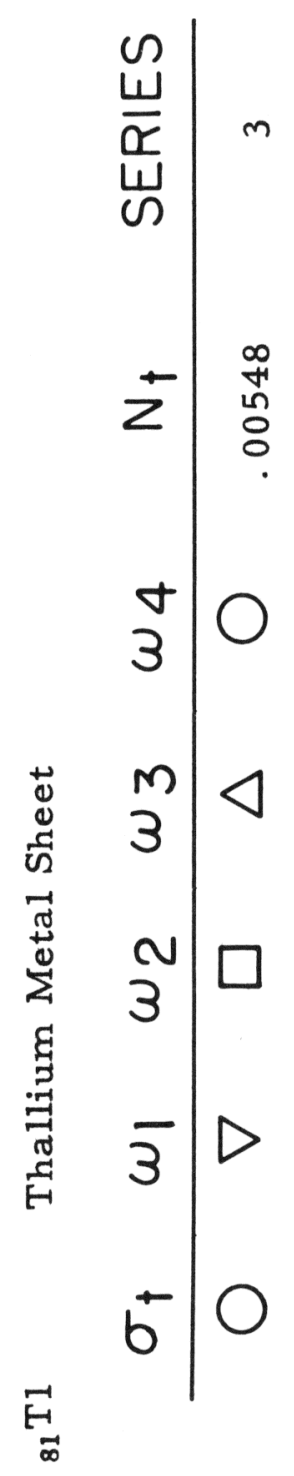




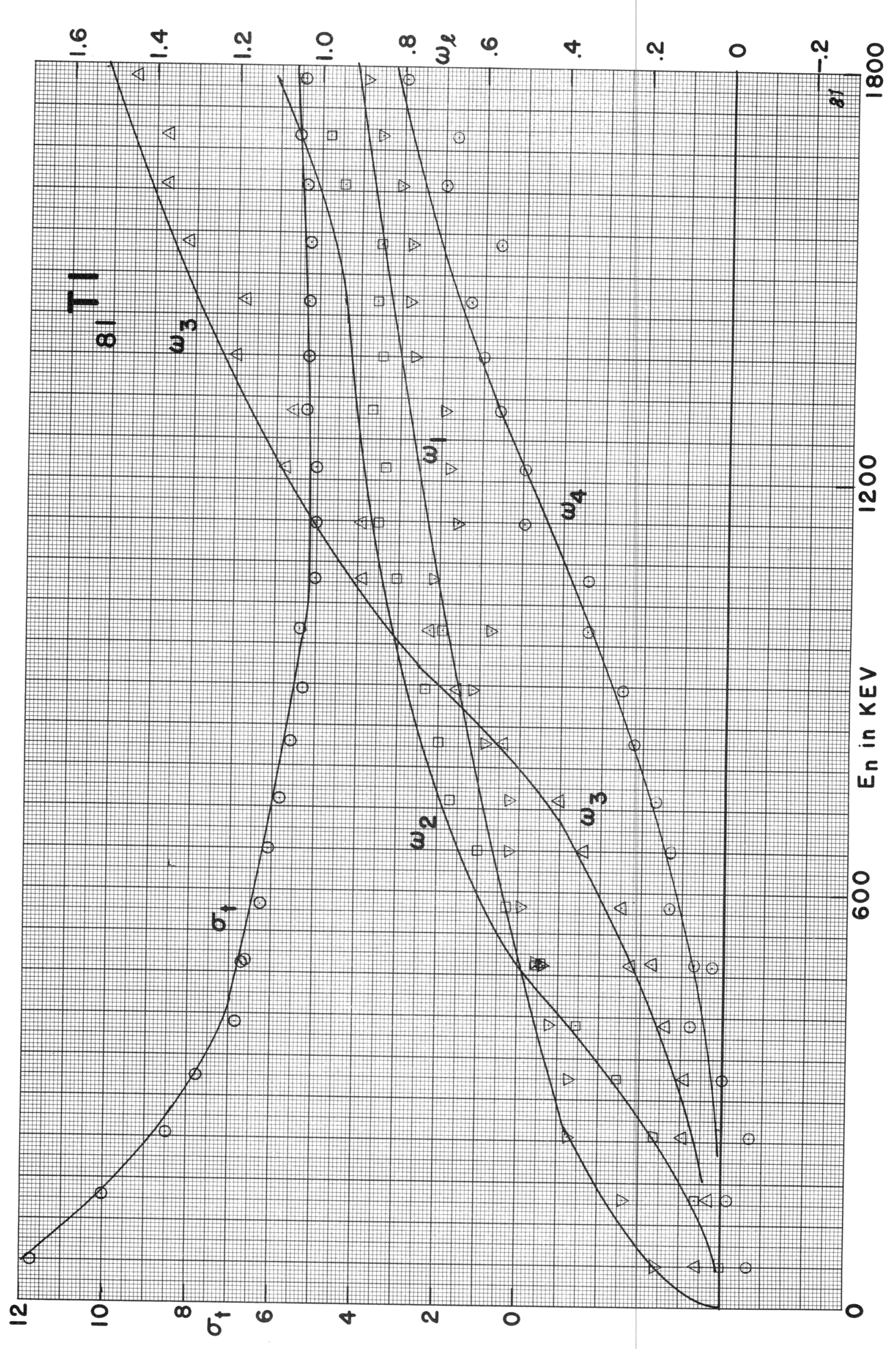




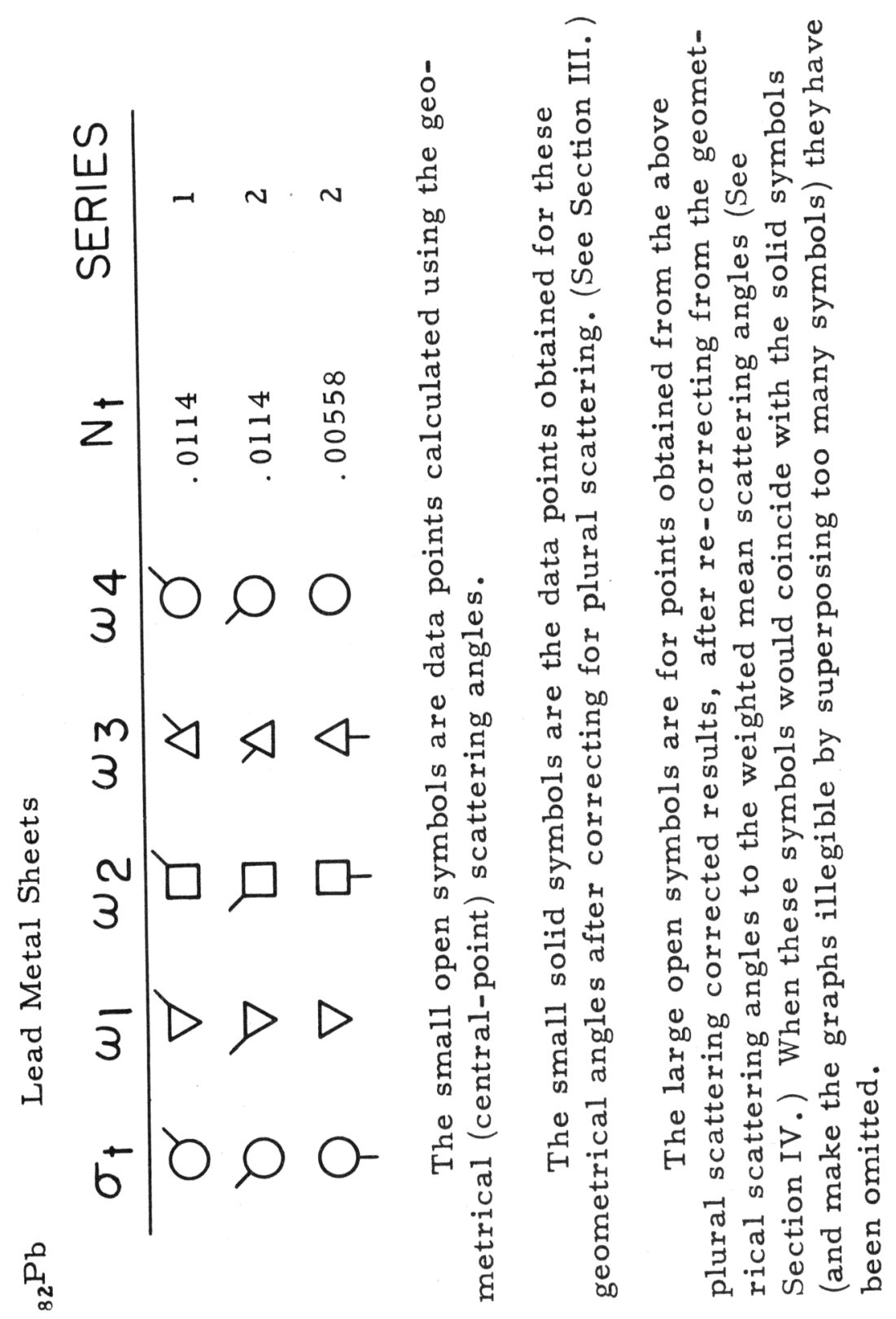




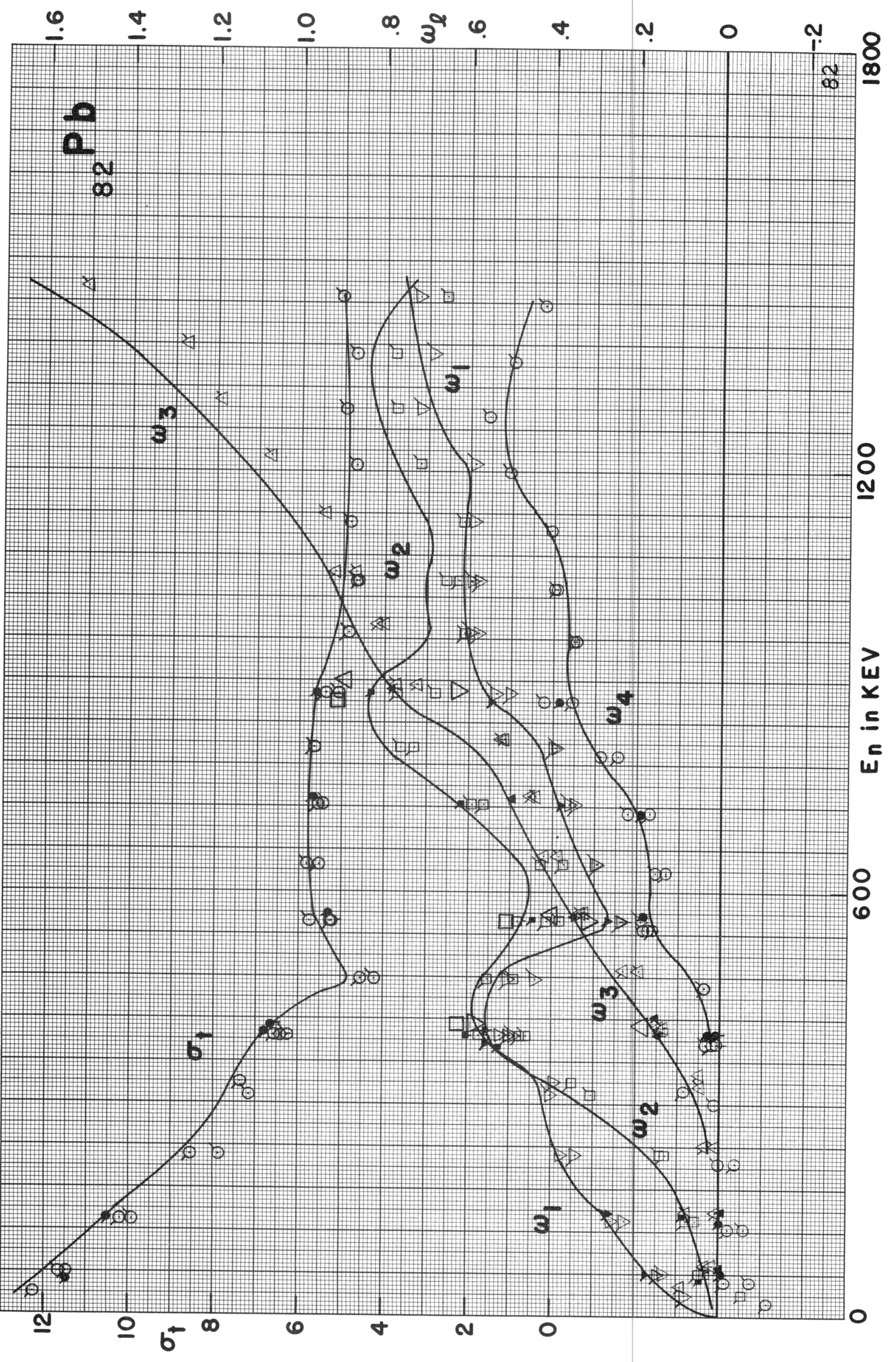




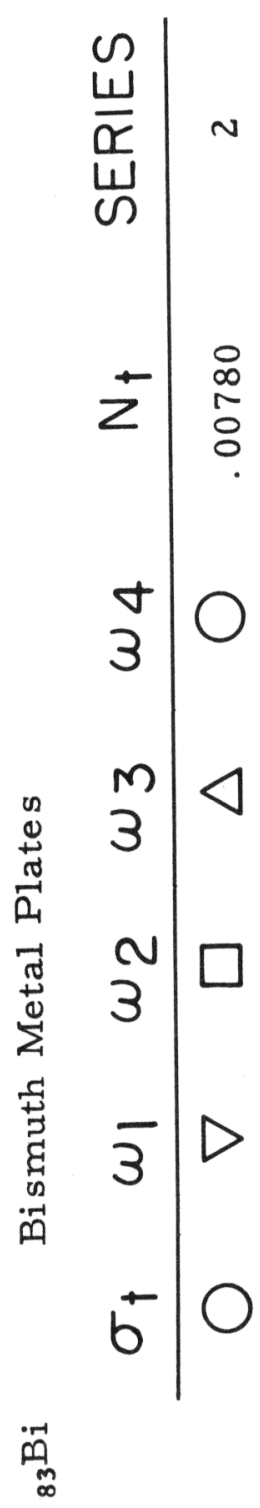




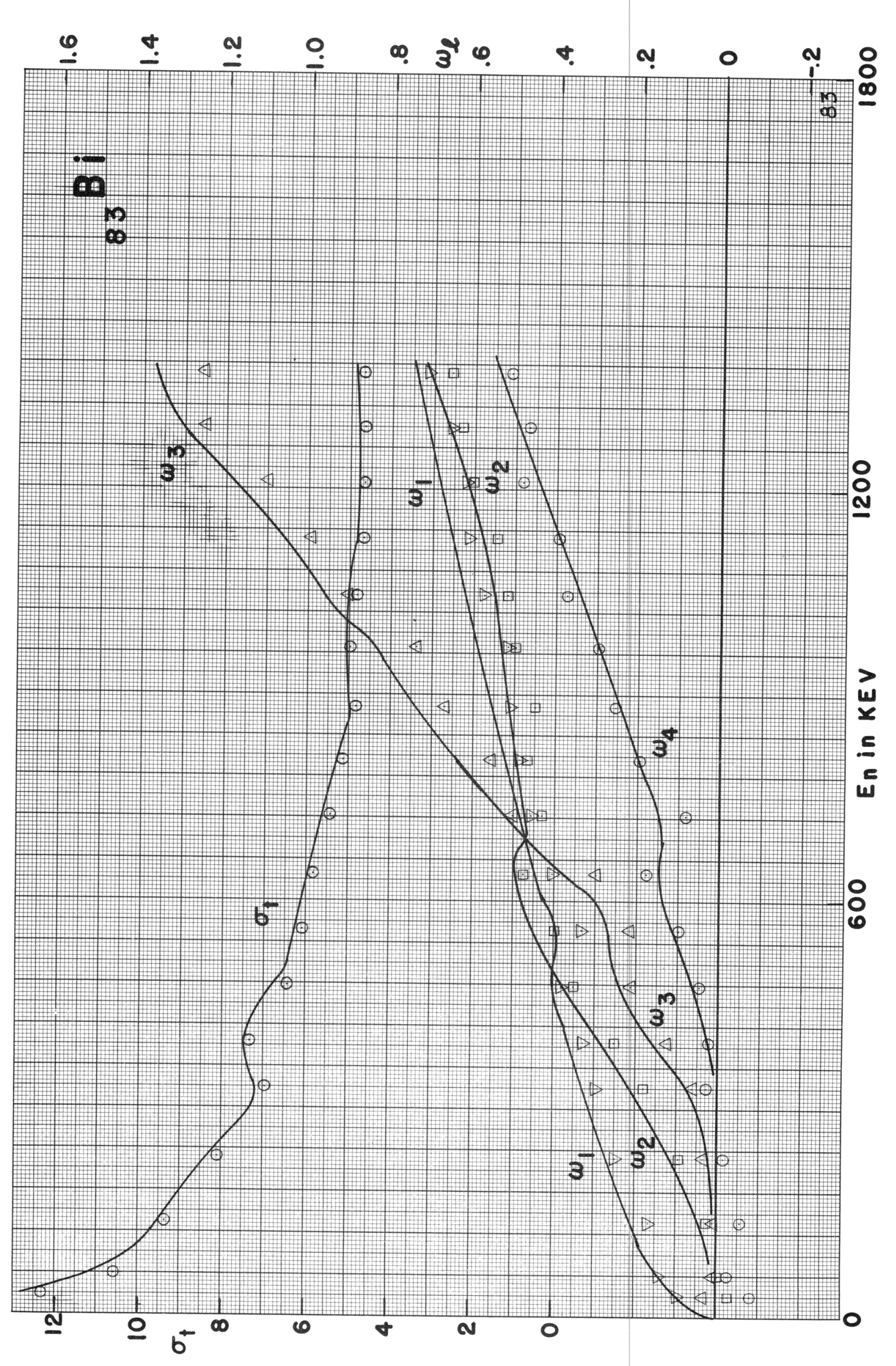




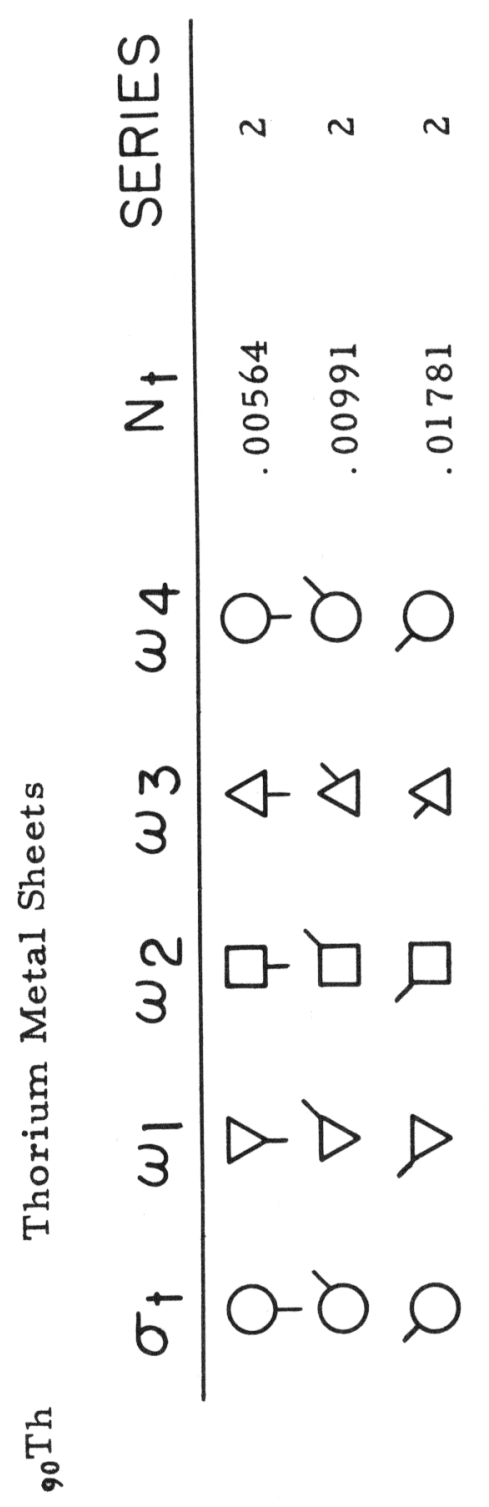




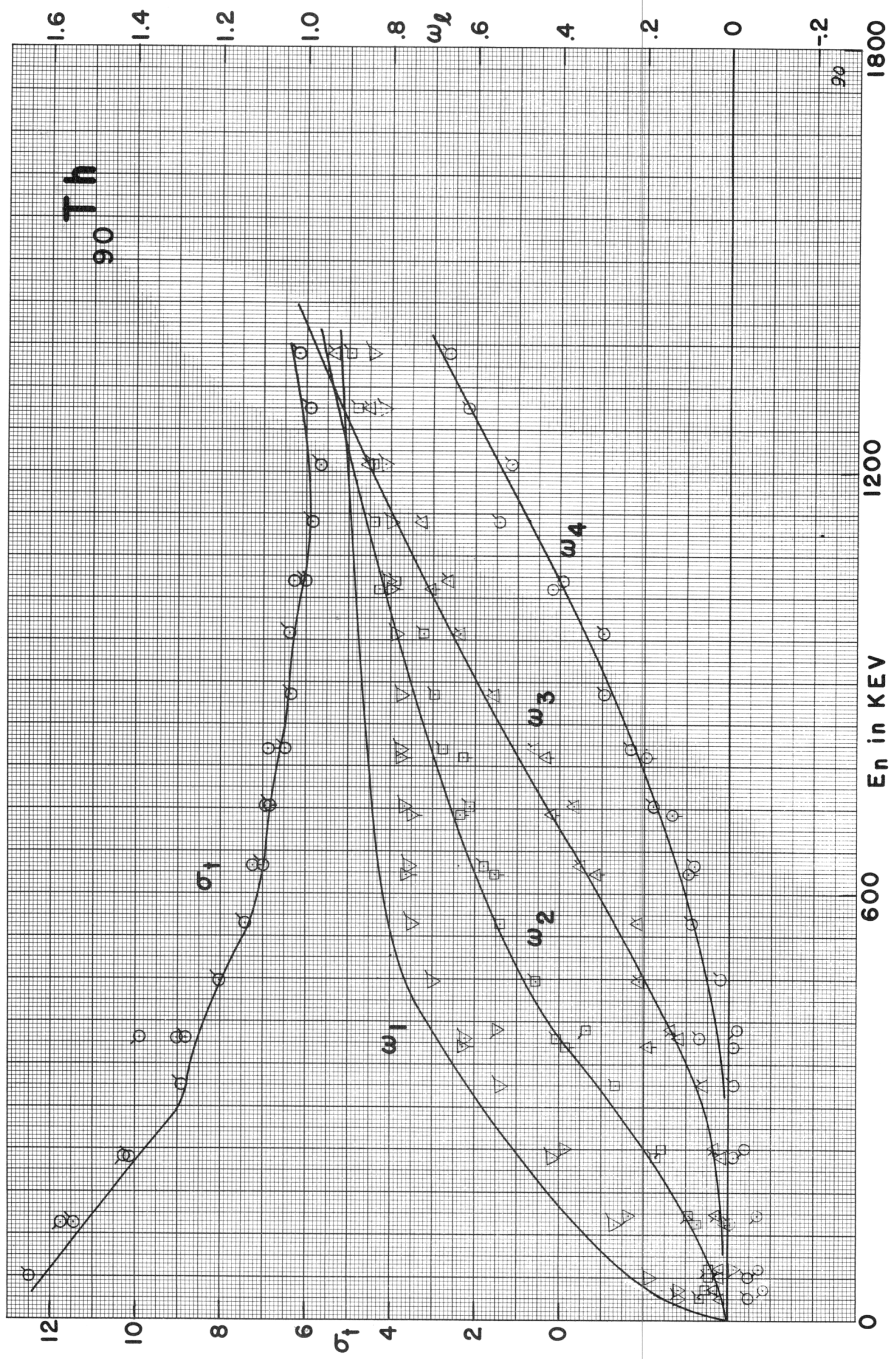




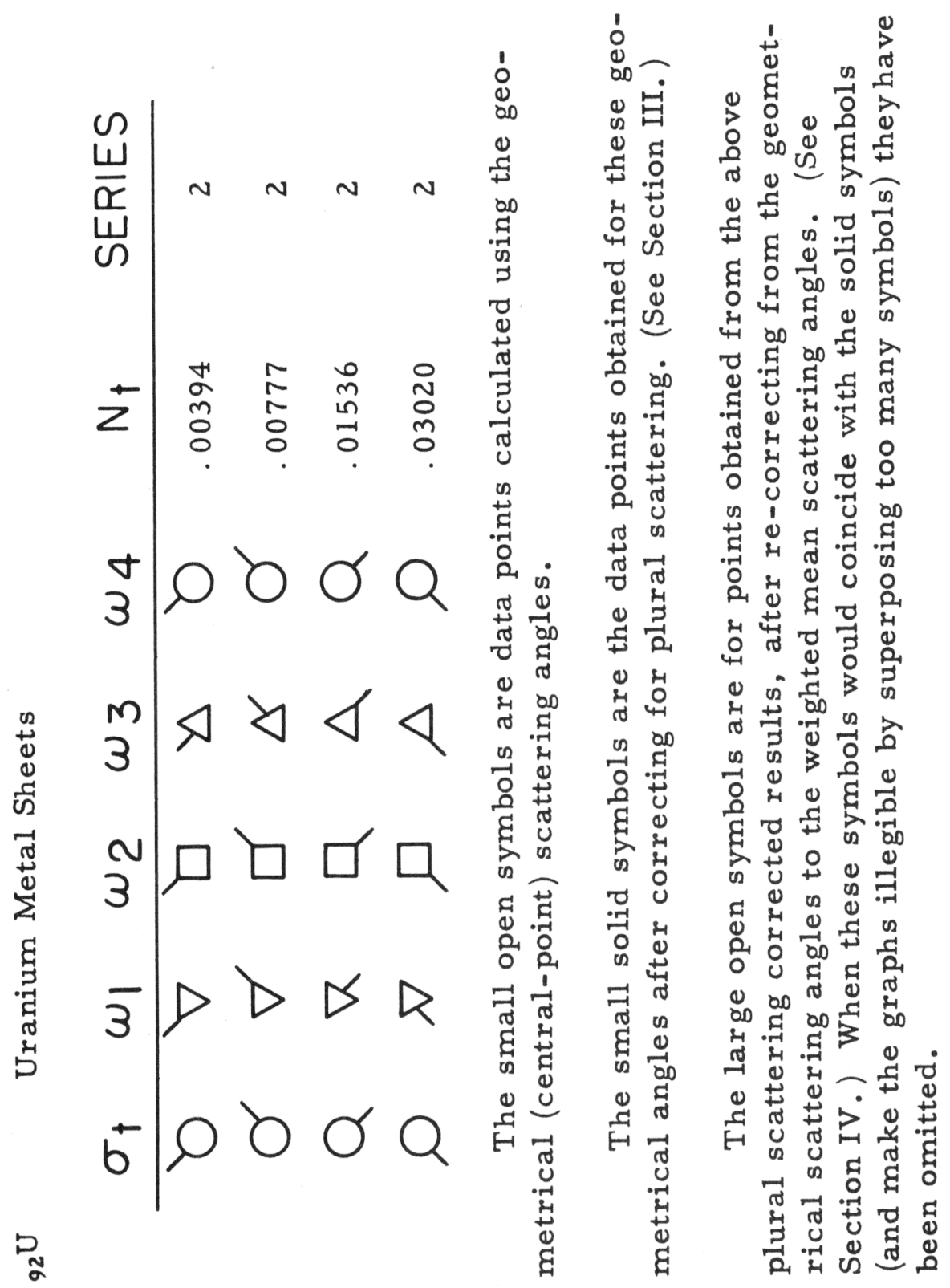




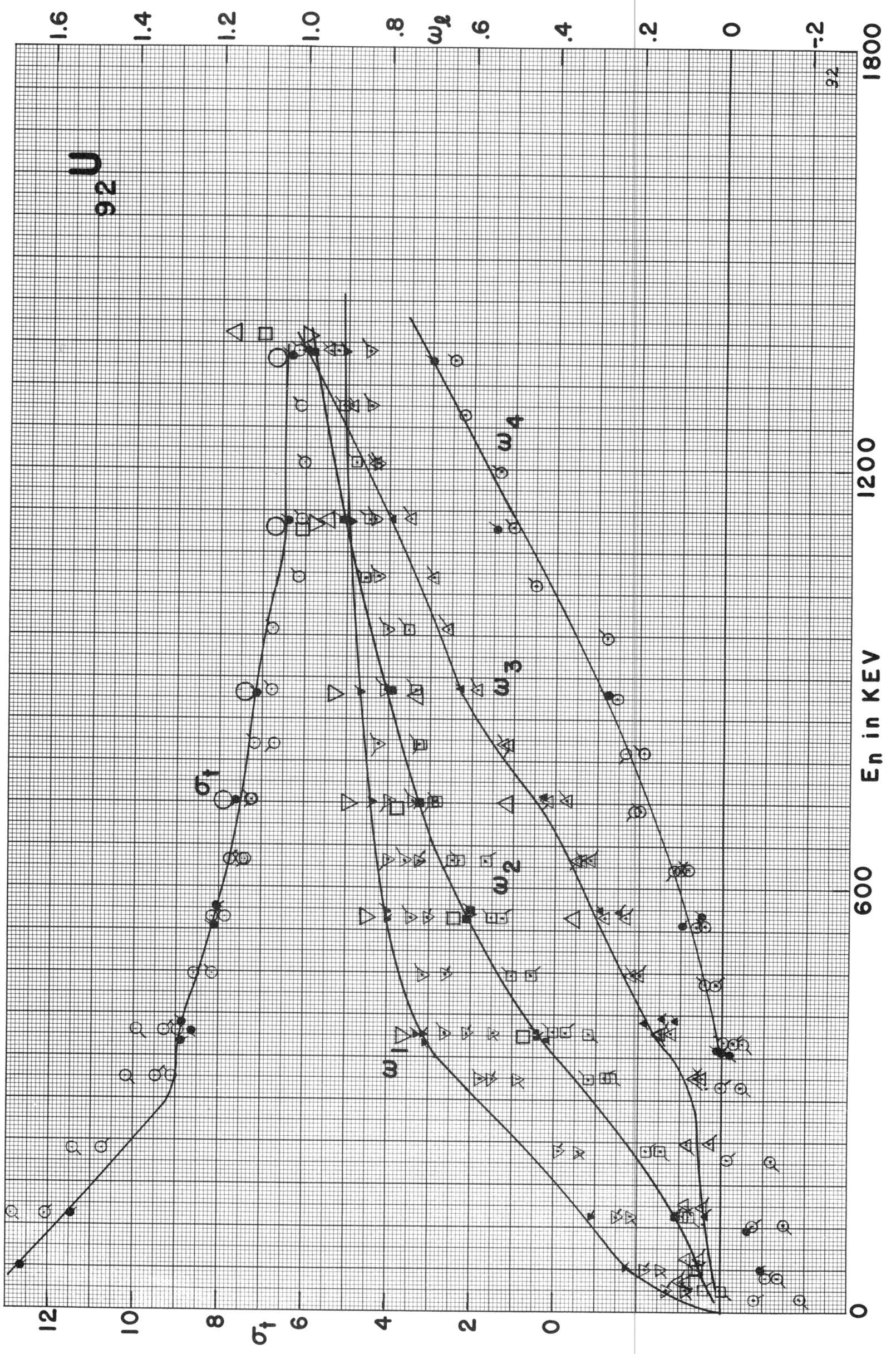




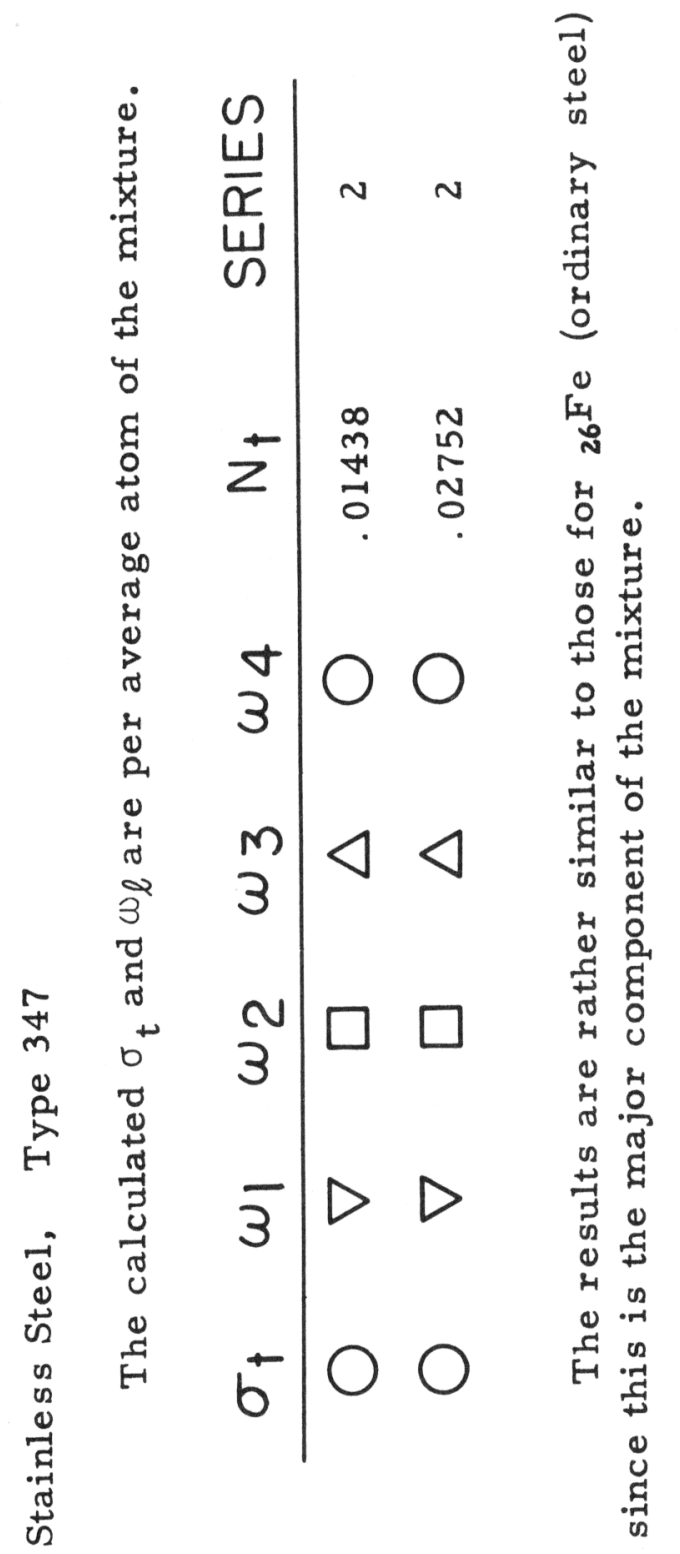




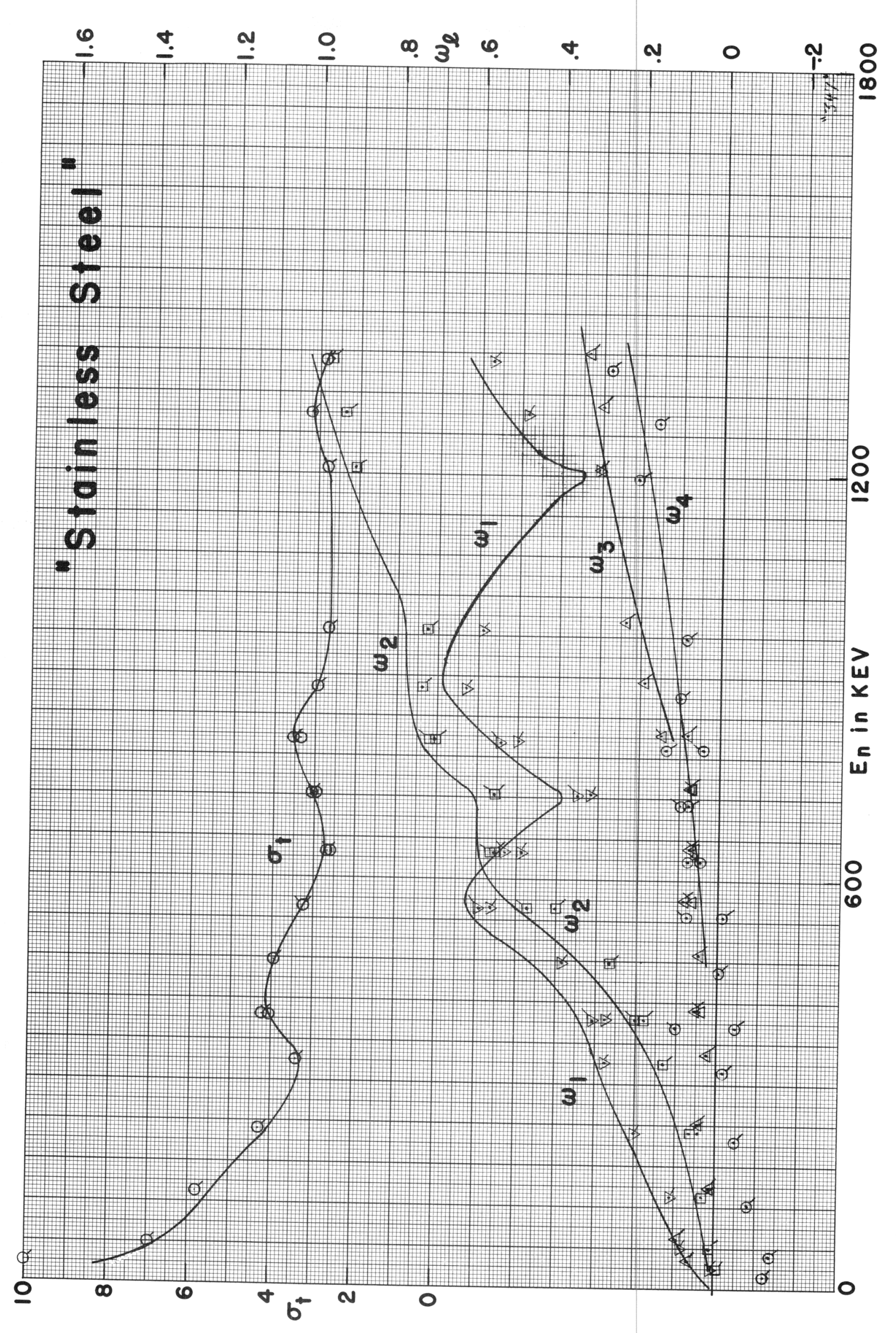




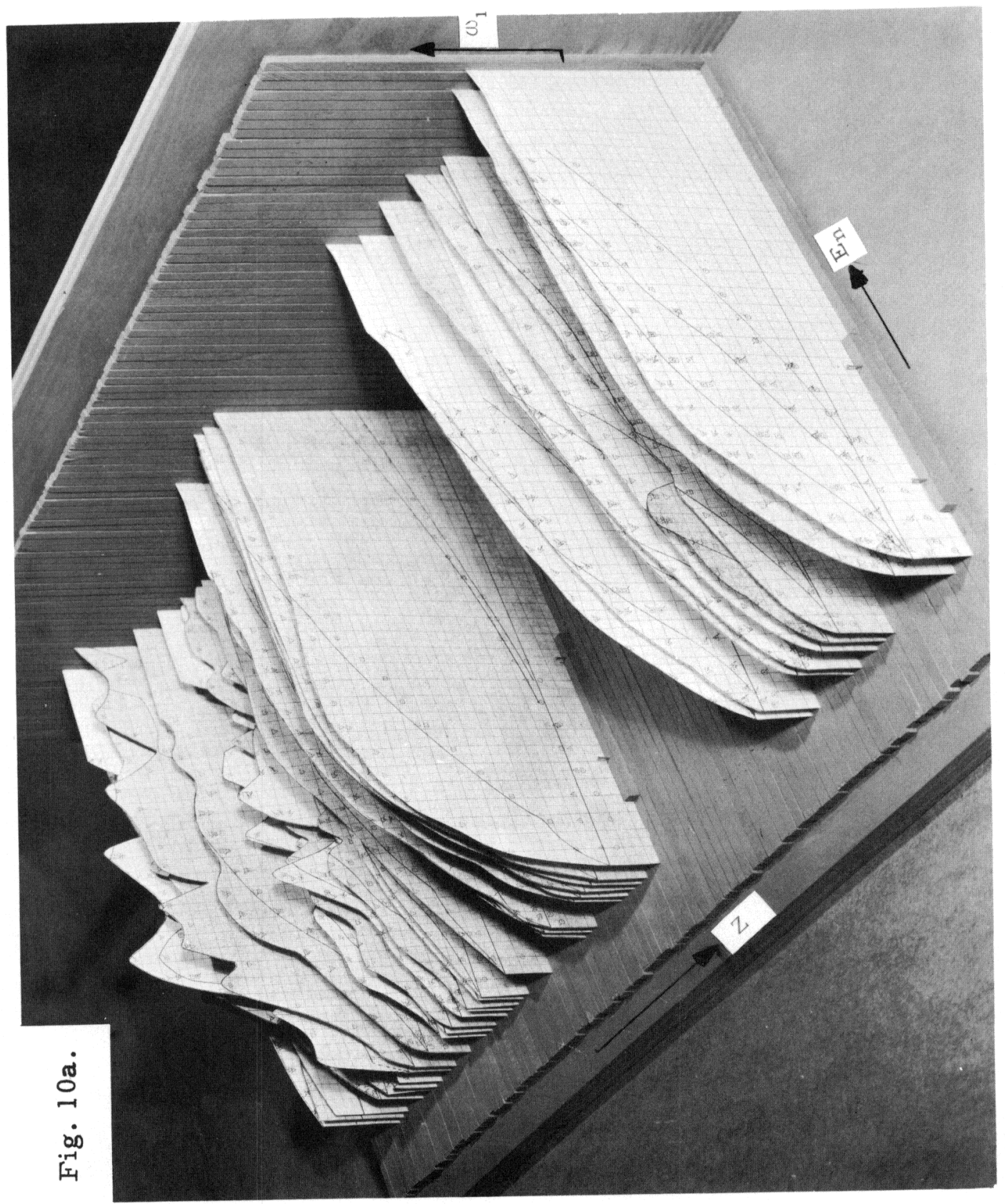




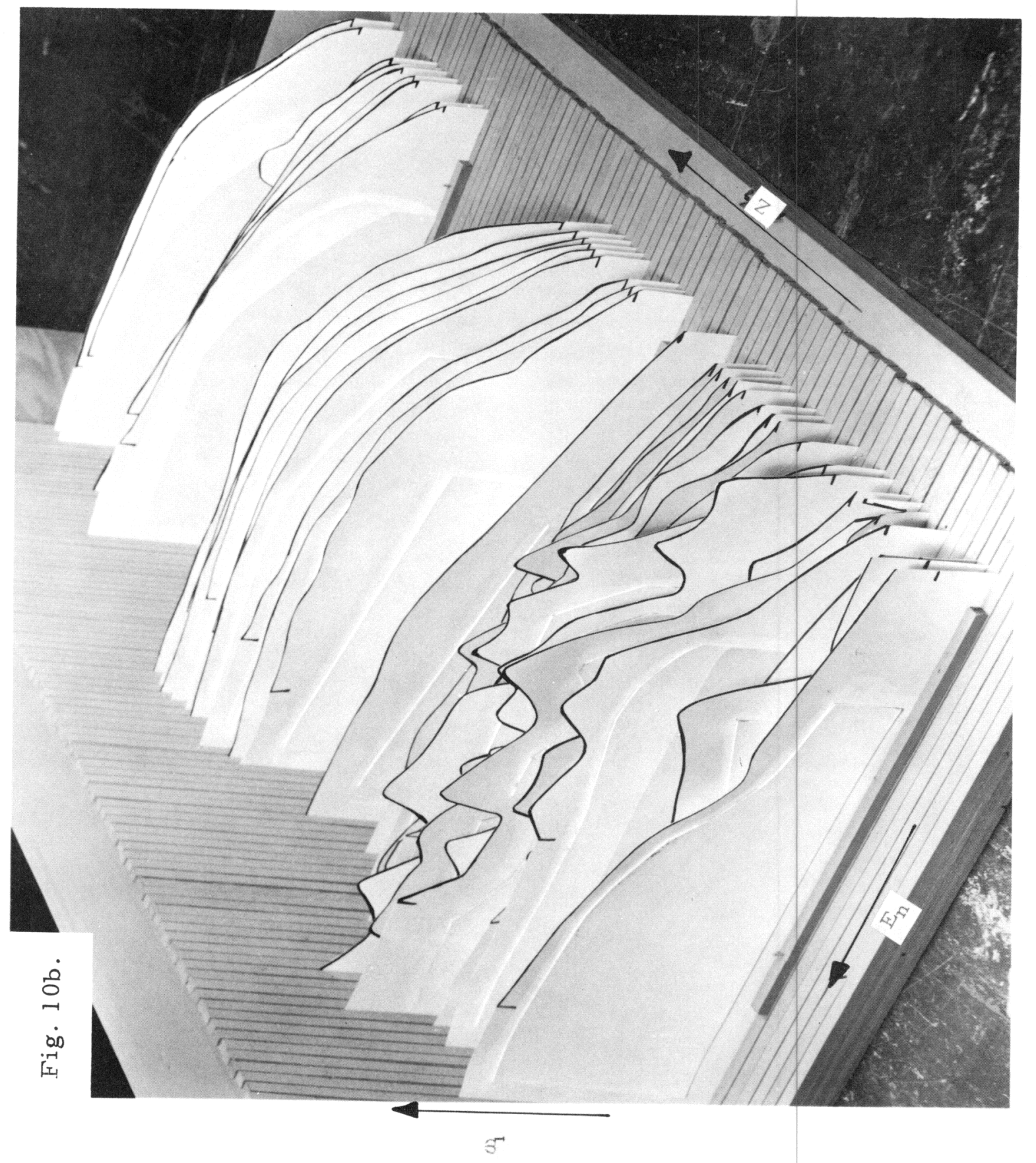

पै山 4

0

o. 5

กี

4

\&.

$+7$

언

국

$\therefore$.

कo "0

\&

(4)

पै

द्व

号过

苞

त

( )

त द्व

उे

$+3$

秀

总

0

o

ฐ

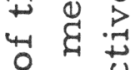

4 त 0

. 0 -

के $\beta$

(1)

(1) 0

㝴

in

\&

얼

ज द्व

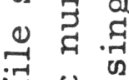

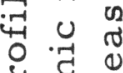

\& द्व

ष 


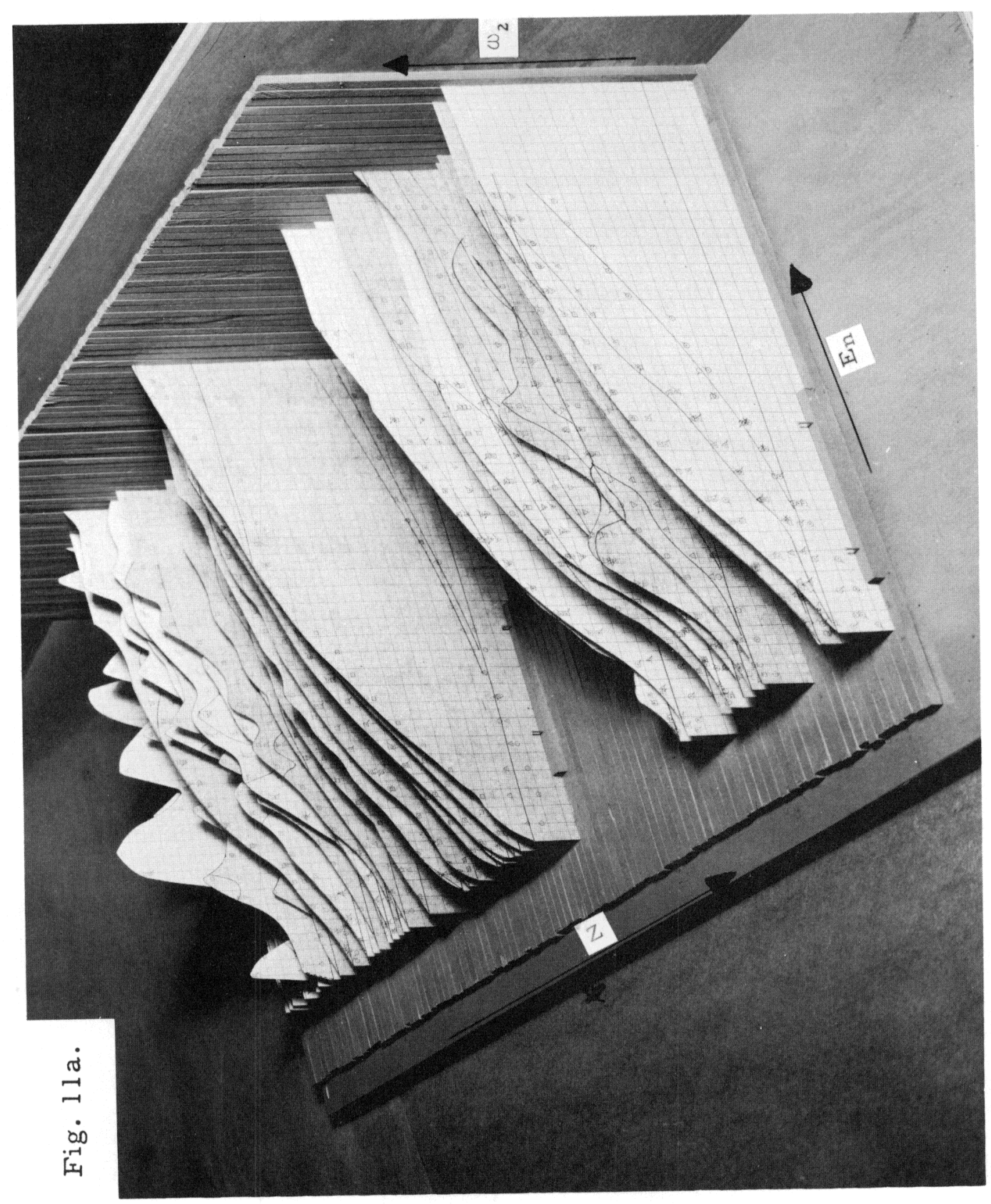




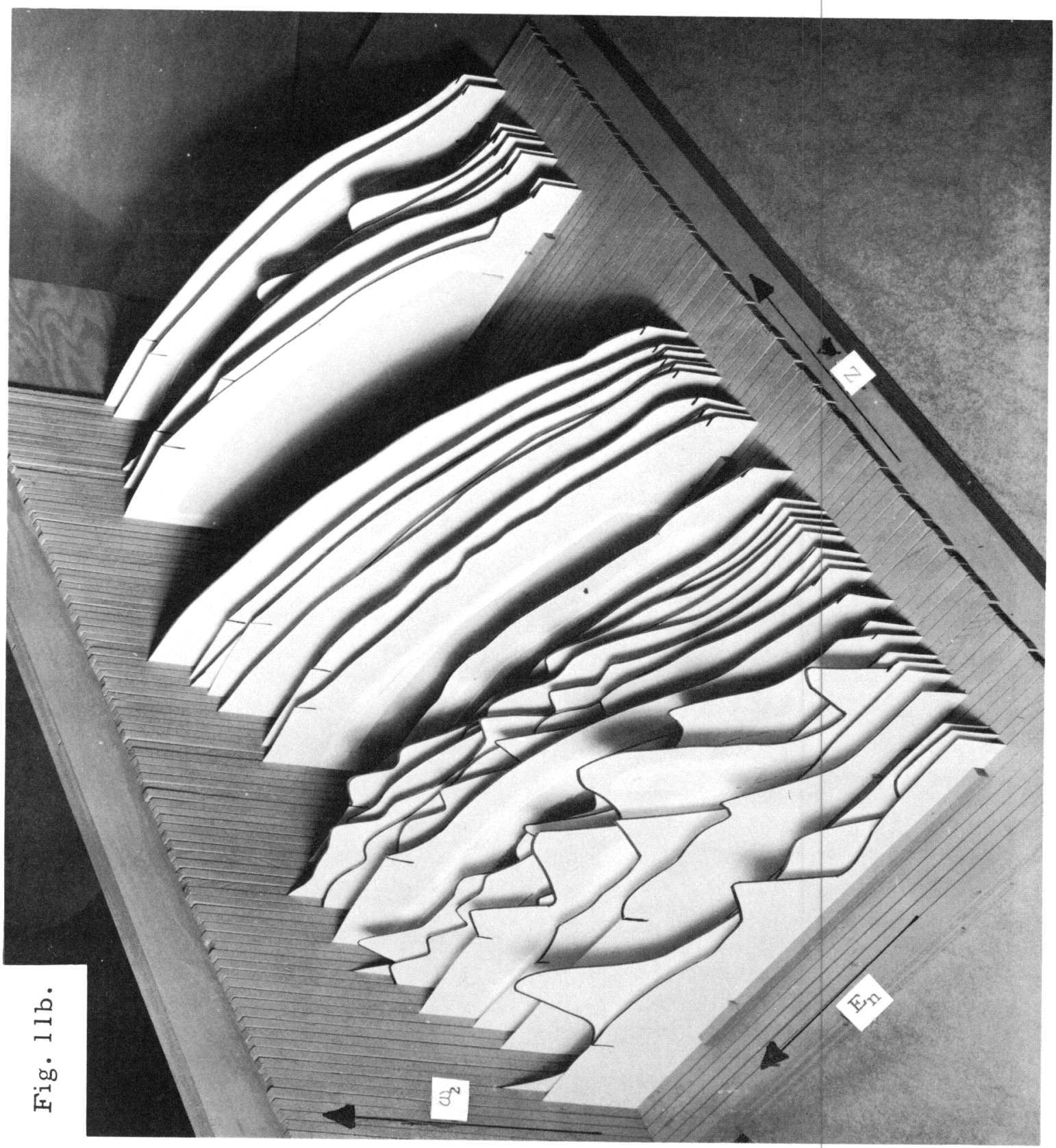

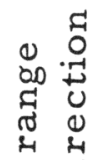

(1) न्ठ

곤

म 昰

애.

दे म्ञ

जी.

$\therefore \circ$

o

i. 3

द 0

(1) 2

फ

द

F

U

?

(ช)

त 0

त

3

苨

-

展

(1)

유

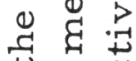

प

क न्दु थ

$\circ 3$

is

空

๑) 00

(1)

क्षे

का म

I 0 i

- 3

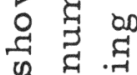

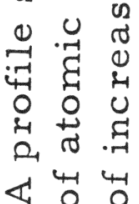




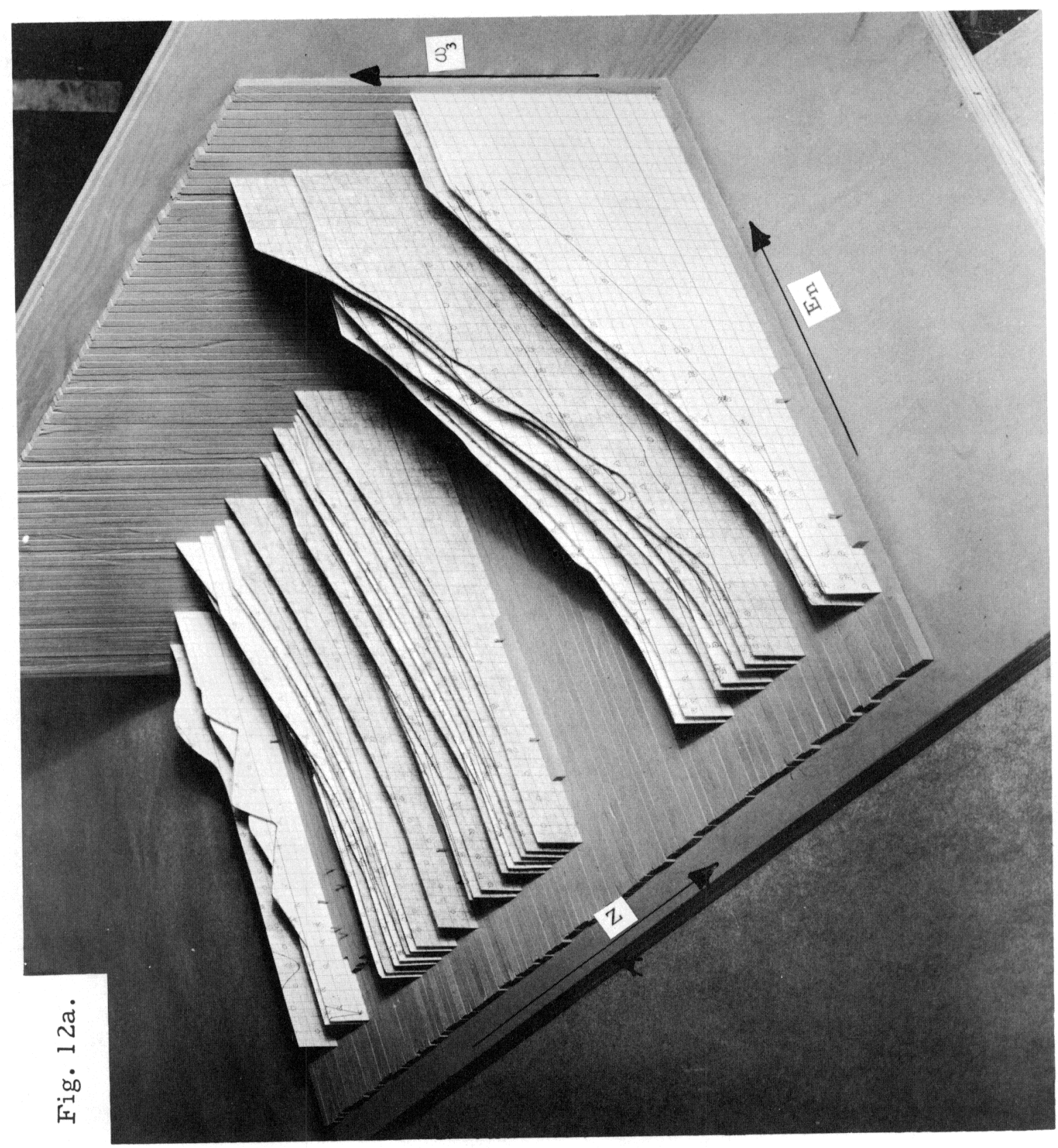



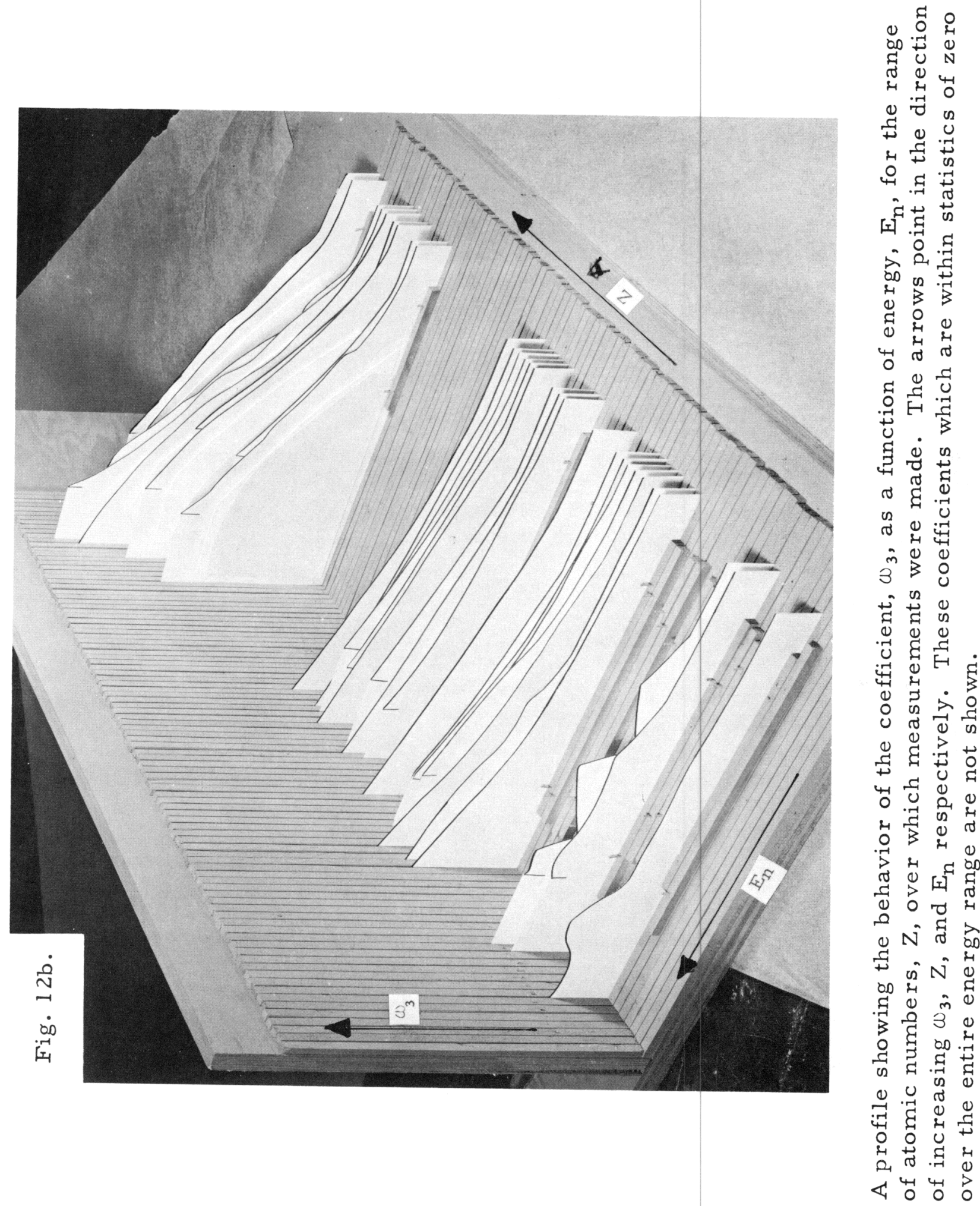


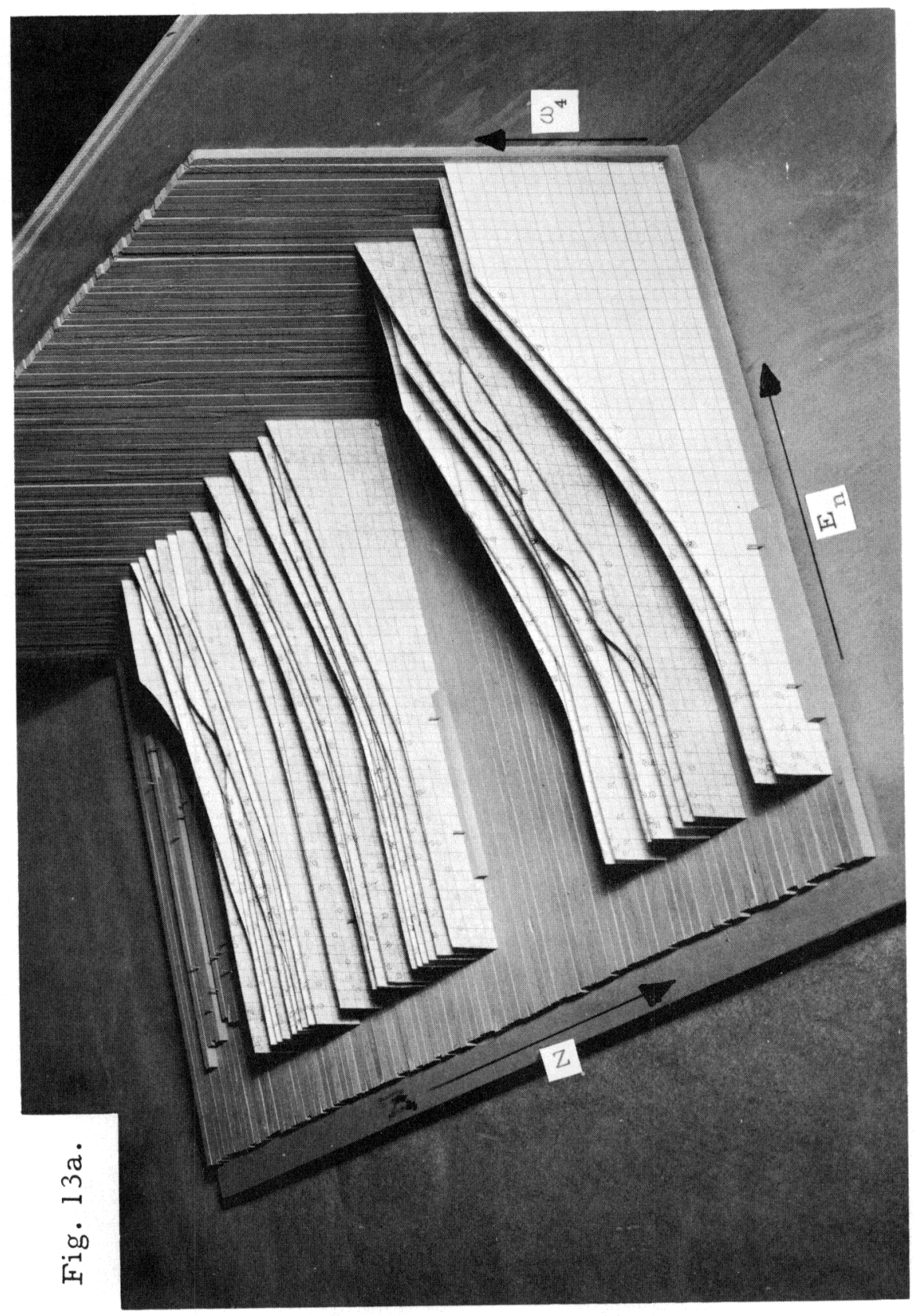



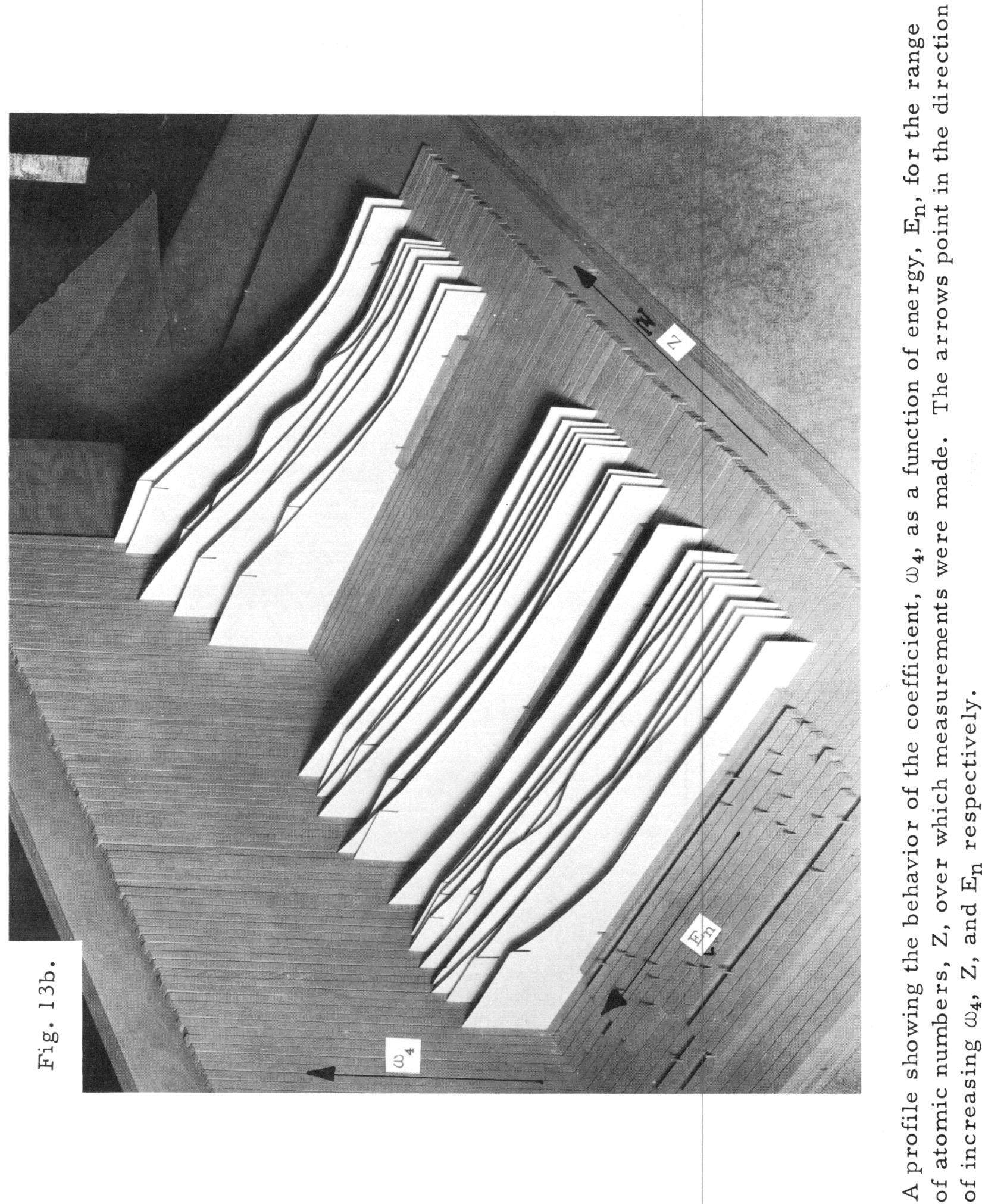


\section{APPENDIX A}

Some Generalizations of the Exponential Integrals

Placzek ${ }^{1}$ defines the functions $E_{n}(x)$ by the integral

$E_{n}(x)=\int_{1}^{\infty} e^{-x u} d u / u^{n}$.

Many properties of these functions, and integrals whose solutions involve these functions, are given in the tabulations by Placzek, ${ }^{1}$ and by Le Caine. ${ }^{2}$

Chandrasekhar 3 and van de $\mathrm{Hulst}^{4}$ discuss the function they call $E_{1}^{(2)}(x)$ which is defined by the integral

$E_{1}^{(2)}(x)=\int_{x}^{\infty} E_{1}(x) d x / x$

This same function is also to be found incorporated in Le Caine and in the much earlier work by Nielsen. 5

Kourganoff ${ }^{6}$ shows that the function $E_{1}^{(2)}(x)$ may be written as the definite integral

$$
E_{1}^{(2)}(x)=\int_{1}^{\infty} e^{-x u} \log u d u / u
$$

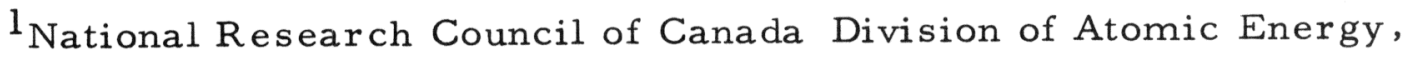

Chalk River, Ontario, Report N.R.C. No. 1547, MT-1, by

G. Placzek: The Functions $E_{n}(x)$.

2 National Research Council of Canada Atomic Energy Project, Chalk River, Ontario, Report N.R.C. No. 1553, MT-131, by $\mathrm{J}$. LeCaine: A Table of Integrals Involving the Functions $\mathrm{E}_{\mathrm{n}}(\mathrm{x})$.

${ }^{3}$ S. Chandrasekhar. Radiative Transfer. Oxford Clarendon Press 1950.

4H. C. van de Hulst, Astro. phys. J. 107, 220 (1948).

${ }^{5}$ Nielsen. Theorie des Integral logarithmus, Leipzig, 1906.

6V. Kourganoff, Basic Methods in Transfer Problems, Oxford, 1952, page 258 et seq. 
None of these authors discuss a generalization of these functions to a larger class or for negative arguments. Only for $E_{1}(x)$ is the problem considered; in this case Chandrasekhar and others make use of the well-known functions written as $\overline{\mathrm{Ei}}(\mathrm{x})$ by Jahnke-Emde. $?$

The function which we will define, for positive arguments, and positive integer $m$ and $n$,

$$
E_{n}^{m}(x)=\int_{1}^{\infty} e^{-x u}(\log u)^{m-1} d u / u^{n}
$$

includes the entire class of functions defined by Placzek and the other authors referred to above. We will also show that the functions $E_{n}^{m}(x)$ can be defined for negative as well as positive arguments and that infinite series expressions and recursion relations can be derived for the entire class.

The defining equation (A4) for $E_{n}^{m}(x)$ may be integrated by parts to give, after a slight rearrangement,

$$
E_{n}^{m+1}(x)=\frac{m E_{n}^{m}(x)-x E_{n-1}^{m+1}(x)}{n-1}, \begin{array}{r}
n>1 \\
m>0
\end{array} .
$$

If $\mathrm{m}=0$, the above recurrence relation is replaced by the one given by Placzek,

$$
E_{n}^{1}(x)=\frac{e^{-x}-x E_{n-1}^{1}(x)}{n-1} \quad n>1
$$

The above recurrence relations make it clear that if $E_{1}^{m}(x)$ are known for all $\mathrm{m}$ up to some specified $\mathrm{m}$, and all $\mathrm{E}_{\mathrm{n}}^{1}(\mathrm{x})$ are known, then the application of Eq. (A5) in principle permits the evaluation of all $E_{n}^{m}(x)$ up to the specified $m$. For positive arguments, many $E_{n}^{1}(x)$ are available in the tabulation by Placzek.

For $\mathrm{n}=1$ but general $\mathrm{m}$, an integration by parts yields

$$
\begin{aligned}
E_{1}^{m}(x) & =\int_{1}^{\infty} e^{-x u}(\log u)^{m-1} d u / u \\
& =\frac{x}{m} \int_{1}^{\infty} e^{-x u}(\log u)^{m} d u=\frac{x}{m} E_{0}^{m+1}(x) .
\end{aligned}
$$

${ }^{7}$ E. Jahnke and F. Emde, Third Edition (page 2), Tables of Functions,

G. E. Stechert and Co., New York. (Teubner, Leipzig, 1938). 
We may now write

$$
E_{1}^{m}(x)=\frac{x}{m} \int_{0}^{\infty} e^{-x u}(\log u)^{m} d u-\frac{x}{m} \int_{0}^{1} e^{-x u}(\log u)^{m} d u
$$

The final integral above may be written

$$
\mathrm{w}_{1}^{\mathrm{m}}(\mathrm{x})=\frac{\mathrm{x}}{\mathrm{m}} \int_{0}^{1} \mathrm{e}^{-\mathrm{xu}}(\log \mathrm{u})^{\mathrm{m}} \mathrm{du}=\int_{0}^{1} \frac{\mathrm{e}^{-\mathrm{xu}}-1}{u}(\log u)^{\mathrm{m}-1} \mathrm{du}
$$

so that

$$
\mathrm{w}_{1}^{\mathrm{m}+1}(\mathrm{x})=-\mathrm{m} \int_{0}^{\mathrm{x}} \mathrm{w}_{1}^{\mathrm{m}}\left(\mathrm{x}^{\prime}\right) \mathrm{d} \mathrm{x}^{\prime} / \mathrm{x}^{\prime}
$$

An expansion of the expression for $W_{1}^{1}(x)$ gives

$$
W_{1}^{1}(x)=\sum_{n=1}^{\infty} \frac{(-x)^{n}}{n \cdot n !}
$$

and by continuation one finds

$$
\mathrm{W}_{1}^{\mathrm{m}}(\mathrm{x})=(-)^{\mathrm{m}-1}(\mathrm{~m}-1) ! \sum_{\mathrm{n}=1}^{\infty} \frac{(-\mathrm{x})^{\mathrm{n}}}{\mathrm{n}^{\mathrm{m}} \cdot \mathrm{n} !}
$$

The value of the remaining integral in $\mathrm{Eq}$. (A8) for $\mathrm{E}_{\mathbf{1}}^{\mathrm{m}}(\mathrm{x})$ can be determined as follows:

Le Caine uses the relation for the derivatives of the gamma function:

$$
\left[\frac{\mathrm{d}^{\mathrm{n}} \Gamma(\mathrm{z})}{\mathrm{d} \mathrm{z}^{\mathrm{n}}}\right]_{\mathrm{Z}=1}=\int_{0}^{\infty} \mathrm{e}^{-\mathrm{t}}(\log \mathrm{t})^{\mathrm{n}} \mathrm{dt}
$$

to evaluate

$$
\int_{0}^{\infty} e^{-x u}(\log u)^{m} d u
$$

up to $\mathrm{m}=4$.

It is clear results such as Le Caine gives may be extended, with sufficient labor, to as large an $\underline{m}$ as may be desired. Therefore one can construct, in principle, tables of numerical values of $\mathrm{E}_{\mathrm{n}}^{\mathrm{m}}(\mathrm{x})$, 
at least for positive argument, to any extent desired. Of course, for a very large argument, $x$, it would be necessary to find asymptotic expressions because the functions $W_{1}^{m}(x)$ become very slowly convergent for large $\mathrm{x}$. relations

For the functions $\mathrm{E}_{\mathrm{n}}^{\mathrm{m}}(\mathrm{x})$ the integral and derivative recurrence

$$
\begin{aligned}
& E_{n+1}^{m}(x)=\int_{x}^{\infty} E_{n}^{m}\left(x^{\prime}\right) d x^{\prime} \\
& E_{n+1}^{\prime m}(x)=-E_{n}^{m}(x)
\end{aligned}
$$

follow by simple integration and differentiation.

In the case of Placzek's $\mathrm{E}_{\mathrm{n}}^{1}(\mathrm{x})$, one may consider that

$$
E_{0}^{1}(x)=\frac{e^{-x}}{x}
$$
is a kernel from which all $E_{n}^{1}(x)$ can be derived. Similarly for a general
$\underline{m}$, we observe that

$$
\begin{aligned}
E_{1}^{m}(x) & =\int_{1}^{\infty} e^{-x u}(\log u)^{m-1} d u / u=\int_{1}^{\infty} e^{-x u} d(\log u)^{m} / m \\
& =\frac{x}{m} \int_{1}^{\infty} e^{-x u}(\log u)^{m} d u=\frac{x}{m} E_{0}^{m+1}(x) .
\end{aligned}
$$

This relation leads to the concept that for each order $\underline{m+l}$, the kernel may be considered to be $\mathrm{E}_{0}^{\mathrm{m}+1}(\mathrm{x})$ with

$$
E_{0}^{m+1}(x)=\frac{m E_{1}^{m}(x)}{x}
$$

so that, for example, we may write

$$
E_{1}^{m+1}(x)=\int_{x}^{\infty} E_{0}^{m+1}\left(x^{\prime}\right) d x^{\prime}=m \int_{x}^{\infty} \frac{E_{1}^{m}\left(x^{\prime}\right)}{x^{\prime}} d x^{\prime}
$$

which is the direct connection between orders, $\underline{m}$, of the functions $E_{n}^{m}(x)$.

It will turn out that all the integral, derivative, and recursion relations above apply equally well when the functions have been generalized to negative arguments. Only the defining integrals so far employed have to be abandoned in defining the $\mathrm{E}_{\mathrm{n}}^{\mathrm{m}}(\mathrm{x})$ for $\mathrm{x}$ negative. 
We will now construct a complex generating function for the series expansions of all $E_{n}^{m}(x)$. We find that the complex function

$$
\xi_{n}(z, x)=(-)^{n-1} \frac{(x)^{n-z} \Gamma(z)}{(2-z)(3-z) \ldots(n-z)}+\sum_{\substack{r=0 \\ r \neq n-1}}^{\infty} \frac{(-x)^{r}(r+1-n)}{r !(r+z-n)} \text { (A21) }
$$

has the property

$$
\frac{d \varepsilon_{n}(z, x)}{d x}=-\varepsilon_{n-1}(z, x)
$$

consistent with the derivative relation previously derived for the functions $\mathrm{E}_{\mathrm{n}}^{\mathrm{m}}(\mathrm{x})$, as in Eq. (Al6).

This function also has the property that

$$
\left[\frac{d^{m} \xi_{n}(z, x)}{d z^{m}}\right]_{z=1}=E_{n}^{m}(x) \text {. }
$$

That this is so follows from use of the expressions for the incomplete gamma function, as given, for example, in Jahnke-Emde. 8

From the expression there given, which may be written

$\frac{(\mathrm{x}, \mathrm{y}) !}{(\mathrm{x}, \infty) !}=\frac{1}{\mathrm{x} !} \int_{0}^{\mathrm{y}} \mathrm{e}^{-\mathrm{t}} \mathrm{t}^{\mathrm{x}} \mathrm{dt}=\frac{\mathrm{y}^{\mathrm{x}+1}}{(\mathrm{x}+1) !} \mathrm{r} \stackrel{\infty}{\stackrel{\infty}{\infty}} \frac{(-\mathrm{y})^{\mathrm{r}}(\mathrm{x}+1)}{\mathrm{r} !(\mathrm{x}+\mathrm{r}-1)}$

using also $\Gamma(x+1)=x$, ,

and the definition

$$
\Gamma(x+1, y)=(x, y) !,
$$

we rewrite

$$
\int_{y}^{\infty} e^{-t} t^{z-1} d t=\Gamma(z)-\Gamma(z, y)
$$

where

$$
\Gamma(z, y)=y^{z} \quad \sum^{\infty}=0 \frac{(-y)^{r}}{r !(r+z)}
$$

${ }^{8}$ Ibid. (page 22). 
Substituting $t=-x v$ and $y=-x$, one obtains

$$
(-x)^{z} \int_{1}^{\infty} e^{x v} v^{z-1} d v=\Gamma(z)-\Gamma(z,-x)
$$

and finally,

$$
-x \int_{1}^{\infty} e^{x v} v^{z-1} d v=(-x)^{1-z} \Gamma(z)+\sum_{r=1}^{\infty} \frac{x^{r} r}{r !(r+z-1)}
$$

This equation leads to the expression for $\varepsilon_{n}(z, x)$ of Eq. (A21) by repeated integrations over $\mathrm{x}$ from $\mathrm{x}$ to infinity .

The actual evaluation of expansions for $\mathrm{E}_{\mathrm{n}}^{\mathrm{m}}(\mathrm{x})$ by this generating function method involves some interesting properties related to the technique of differentiation, having to do with the use of logarithmic derivative to evaluate the derivatives of the product term involving the gamma function. We define

$$
\infty_{n}(z, x)=\frac{x^{1-z} \Gamma(z)}{(2-z) \ldots(n-z)}=\frac{x\left[e^{-z} \log x\right]_{\Gamma}(z)}{(2-z) \ldots(n-z)}
$$

so that

$$
E_{n}(z, x)=(-x)^{n-1} \infty_{n}(z, x)+\sum_{r=0}^{\infty} \frac{(-x)^{r}(r+1-n)}{r(r+z-n)} .
$$

Then

$$
\log \mathcal{P}_{n}(\mathrm{z}, \mathrm{x})=-\mathrm{z} \log \mathrm{x}+\log \Gamma(\mathrm{z})-\sum_{\mathrm{s}=2}^{\mathrm{n}} \log (\mathrm{s}-\mathrm{z})+\log \mathrm{x} .
$$

We also define the function $\phi_{\mathrm{n}}(\mathrm{z}, \mathrm{x})$ to be the logarithmic derivative of $\boldsymbol{D}_{\mathrm{n}}(z, \mathrm{x})$,

$$
\phi_{\mathrm{n}}(\mathrm{z}, \mathrm{x})=\frac{\mathrm{d} \log \boldsymbol{\rho}_{\mathrm{n}}}{\mathrm{d} \mathrm{z}}=-\log \mathrm{x}+\psi(\mathrm{z})+\sum_{\mathrm{s}=2}^{\mathrm{n}} \frac{1}{\mathrm{~s}-\mathrm{z}}
$$

where $\psi(z)$ is the logarithmic derivative of the gamma function.

Now

$$
\infty_{n}(1, x)=\frac{1}{(n-1) !}
$$




$$
\phi_{n}(1, x)=-\log x-\gamma+{ }_{s}^{n} \sum_{1}^{-1} \frac{1}{s}
$$

so that

$$
\left[\frac{\mathrm{d} P_{\mathrm{n}}(\mathrm{z}, \mathrm{x})}{\mathrm{d} z}\right]_{\mathrm{z}=1}=\infty_{\mathrm{n}}(1, \mathrm{x}) \phi_{\mathrm{n}}(1, \mathrm{x})=\frac{1}{(\mathrm{n}-1)} !\left(-\log \mathrm{x}-\gamma+\sum_{\mathrm{s}=1}^{\mathrm{n}-1} \frac{1}{\mathrm{~s}}\right)
$$

and, finally

$$
\left[\frac{\mathrm{d} \boldsymbol{\varepsilon}_{\mathrm{n}}(\mathrm{z}, \mathrm{x})}{\mathrm{d} \mathrm{z}}\right]_{\mathrm{z}=1}=\sum_{\substack{\mathrm{r}=0 \\ \mathrm{r} \neq \mathrm{n}-1}}^{\infty} \frac{(-\mathrm{x})^{\mathrm{r}}}{\mathrm{r} !(\mathrm{n}-1-\mathrm{r})}-\frac{(-\mathrm{x})^{\mathrm{n}-1}}{(\mathrm{n}-1) !}\left(\gamma+\log \mathrm{x}-\sum_{\mathrm{s}=1}^{\mathrm{n}-1} \frac{1}{\mathrm{~s}}\right)
$$

which in fact agrees exactly with the equation given for $E_{n}(x)$ by Placzek. 9

In the same manner, one may derive the series for $E_{1}^{2}(x)$ given by van de Hulst 10 and, in general, for any $\mathrm{E}_{\mathrm{n}}^{\mathrm{m}}(\mathrm{x})$ that may be desired. To carry through these more complex cases, the material which follows after the next paragraph is useful.

For negative arguments we use a Cauchy principle value to obtain a real value of $\varepsilon_{n}(z,-x)$. We take

$$
\begin{aligned}
& \xi_{n}^{+}(z,-x)=\varepsilon_{n}\left(z, e^{+i \pi} x\right) \\
& \xi_{n}^{-}(z,-x)=\xi_{n}\left(z, e^{-i \pi} x\right)
\end{aligned}
$$

and then define

$$
\xi_{n}(z,-x)=1 / 2 \xi_{n}^{+}(z,-x)+1 / 2 \xi_{n}^{-}(z,-x) \text {. }
$$

The value of $\xi_{n}(z,-x)$ so defined may be shown to yield the same answers for $\mathrm{E}_{\mathrm{n}}^{\mathrm{m}}(\mathrm{x})$ as are given in its series expansion by always reading each logarithm term as the logarithm of the magnitude, e.g., $\log x$ is read as $\log |\mathbf{x}|$.

Some additional material on relations of the derivatives of the gamma functions, useful in evaluating series expansions for exponential integrals, follows:

$$
{ }^{9} \text { Op. cit. }
$$


Following Whittaker and Watson, 11

$\Gamma(z)=\int_{0}^{\infty} e^{-t} e^{(z-1) \log t} d t$.

Thus

$$
\Gamma_{n}=\Gamma_{n}(1)=\left[\frac{d^{n} \Gamma(z)}{d z^{n}}\right]_{z=1}=\int_{0}^{\infty} e^{-t}(\log t)^{n} d t .
$$

We wish to evaluate the $\Gamma_{\mathrm{n}}$ defined as above.

Now

$$
\begin{aligned}
& \psi(z)=\frac{d \ln \Gamma(z)}{d z}=-\gamma-\frac{1}{z}+\sum_{\nu=1}^{\infty}\left(\frac{1}{\nu}-\frac{1}{z+\nu}\right) \\
& \frac{d \psi(z)}{d z}=\sum_{\nu=0}^{\infty} \frac{1}{(z+\nu)^{2}} \\
& \psi_{n}(z)=\frac{d^{n} \psi(z)}{d z^{n}}=(-)^{n+1} n ! \quad \sum_{\nu=0}^{\infty}(z+\nu)^{-n-1}
\end{aligned}
$$

We will abbreviate $\psi_{\mathrm{n}}(1)=\psi_{\mathrm{n}}$ and obtain

$$
\psi_{\mathrm{n}}=(-)^{\mathrm{n}+1} \mathrm{n} ! \zeta(\mathrm{n}+1), \mathrm{n}>1
$$

where $\zeta$ is the Riemann zeta function, except that we define

$$
\zeta(1)=\gamma
$$

where $\gamma$ is the Euler - Macheroni number so that we can relate this special $\zeta(1)$ to

$\psi(1)=\psi_{0}=\psi_{0}(\mathrm{I})=-\gamma$ and apply Eq. (A45) to the case $\mathrm{n}=0$ also.

Now

$$
\Gamma_{1}(z)=\frac{d \Gamma(z)}{d z}=\psi(z) \Gamma(z)
$$

${ }^{11}$ E. T. Whittaker and G. N. Watson, A Course of Modern Analysis, Cambridge (1943); pp 235-280. 
from which

$$
\Gamma_{\mathrm{n}}=\sum_{\mathrm{k}=0}^{\mathrm{n}-1}\left(\begin{array}{c}
\mathrm{n}-1 \\
\mathrm{k}
\end{array}\right) \psi_{\mathrm{k}} \Gamma_{\mathrm{n}-1-\mathrm{k}}
$$

where we have defined $\Gamma_{0}=\Gamma(1)=1$.

This equation can also be written

$$
\Gamma_{n}=\sum_{k=0}^{n-1} \frac{(n-1) !}{(n-1-k) !} \Gamma_{n-1-k}(-)^{k+1} \zeta(k+1) .
$$

If we substitute

$$
\begin{aligned}
& \mathrm{M}_{\mathrm{n}}=\frac{\Gamma_{\mathrm{n}}}{\mathrm{n} !} \\
& \mathrm{N}_{\mathrm{k}}=\frac{\psi_{\mathrm{k}-1}}{\mathrm{k} !}=(-)^{\mathrm{k}} \frac{\zeta(\mathrm{k})}{\mathrm{k}}
\end{aligned}
$$

the equation for $\Gamma_{\mathrm{n}}$ leads to

$$
M_{n}=\sum_{k=1}^{n} \frac{k}{n} N_{k} M_{n-k}
$$

from which it may be shown that

$$
\begin{aligned}
M_{n} & =M_{0} \sum * \frac{N_{1}^{a_{1}}}{a_{1} !} \cdot \frac{N_{2}^{a_{2}}}{a_{2} !} \cdots \cdots \frac{N_{n}^{a_{n}}}{a_{n} !} \\
& =M_{0} \sum * \prod_{i=1}^{n} \frac{N_{1}^{a_{i}}}{a_{i} !}
\end{aligned}
$$

where the sum $\Sigma^{*}$ extends over all possible products with integers $\mathrm{a}_{\mathbf{i}}$ consistent with the condition that

$$
\mathrm{n}=\sum_{\mathrm{i}=1}^{\mathrm{n}} \mathrm{i} \mathrm{a}_{\mathrm{i}}
$$

This is a problem in partitions, such as is discussed in Dickson. 12 This type of solution is valid for more general problems than the particular one of evaluation of gamma function derivatives. It may be used, for example, to obtain detailed solutions for the function $\infty_{n}(1, x)$ in Eq. (A29).

${ }^{12}$ L. E. Dickson, History of the Theory of Numbers, Vol. II, Chelsea Publishing Co. (1952). [Thanks are due to Richard S. Preston for finding this reference.] 


\section{APPENDIX B}

The Functions $F_{j}(\tau, \alpha), F_{j}(\tau, s, \alpha)$, and $M_{0}(\tau, \alpha)$

The Functions $F_{j}(\tau, \alpha)$ and $F_{j}(\tau, s, \alpha)$

The defining equations for the function $F_{j}(\tau, \alpha)$ are

$$
F_{j}(\tau,-\alpha)=\int_{1}^{\infty} \frac{d \beta}{\beta^{j}(\beta+\alpha)}\left[1-e^{-T(\beta+\alpha)}\right]=\int_{0}^{T} d t e^{-\alpha t} E_{j}(t) .
$$

The recursion relation,

$$
F_{j}(\tau,-\alpha)=\frac{1}{\alpha}\left\{\frac{1}{j-1}-e^{-\alpha \tau} E_{j}(\tau)-F_{j-1}(\tau,-\alpha)\right\},
$$

follows directly from the definition since

$$
F_{j}(\tau,-\alpha)=\frac{1}{\alpha} \int_{1}^{\infty} d \beta\left[\beta^{-j}-\beta^{1-j}(\beta+\alpha)^{-1}\right]\left[1-e^{-\tau(\beta+\alpha)}\right] .
$$

This, together with the expression*

$$
F_{1}(\tau, \alpha)=\alpha^{-1}\left[e^{\alpha \tau} E_{1}(\tau)-E_{1}(\tau-\tau \alpha)-\ln |\alpha-1|\right],
$$

permits, at least in principle, the evaluation of $F_{j}(\tau, \alpha)$.

The integral

$$
F_{j}(\tau, s, \alpha)=\int_{0}^{\tau} e^{s x} F_{j}(x, \alpha) d x
$$

occurs frequently in the treatment of double scattering. It can be evaluated by partial integration as

$$
F_{j}(\tau, s, \alpha)=\int_{0}^{\tau} d x \frac{d}{d x}\left(\frac{e^{s x}}{s}\right) F_{j}(x, \alpha)=\frac{1}{s}\left\{e^{s \tau} F_{j}(\tau, \alpha)-F_{j}(\tau, s+\alpha)\right\} .
$$

From this equation it is immediately obvious that

$$
\mathrm{e}^{\mathrm{sh}} \mathrm{F}_{\mathrm{j}}(\mathrm{h},-\mathrm{s}, \mathrm{s}+\alpha)=\mathrm{F}_{\mathrm{j}}(\mathrm{h}, \mathrm{s}, \alpha) .
$$

The Functions $\mathrm{M}_{0}(\tau, \lambda)$

By analogy with Kourganoff's* $M_{0}(\tau)$ - function, we define a two parameter family of integrals

$$
\mathrm{M}_{0}(\tau, \lambda)=\int_{1}^{\infty} \frac{\mathrm{d} \mu}{\mu} \ln (\mu+\lambda) \mathrm{e}^{-\tau \mu} .
$$

*V. Kourganoff, Basic Methods in Transfer Problems, Oxford (1952), Appendix I., page 253 et. seq. 
The Taylor's expansion of $\mathrm{M}_{0}(\tau, \lambda)$ around $\lambda=\lambda_{0}$ is

$$
\mathbf{M}_{0}(\tau, \lambda)=\mathbf{M}_{0}\left(\tau, \lambda_{0}\right)+\sum_{\mathrm{n}=1} \frac{\left(\lambda_{-} \lambda_{0}\right)^{\mathrm{n}}}{\mathrm{n}}(-)^{\mathrm{n}-1} \int_{1}^{\infty} \frac{\mathrm{d} \mu}{\mu\left(\mu+\lambda_{0}\right)^{\mathrm{n}}} \mathrm{e}^{-\tau \mu} .
$$

Since for $\lambda_{0} \geqslant 0$,

$$
\int_{1}^{\infty} \frac{\mathrm{d} \mu \mathrm{e}^{-\tau \mu}}{\mu\left(\mu+\lambda_{0}\right)^{\mathrm{n}}} \leqslant \int_{1}^{\infty} \frac{\mathrm{d} \mu \mathrm{e}^{-\tau \mu}}{\mu^{\mathrm{n}+1}}=\mathrm{E}_{\mathrm{n}+1}(\tau) \leqslant \frac{1}{\mathrm{n}},
$$

we see that the Taylor's series is absolutely convergent for $\lambda-\lambda_{0} \leqslant 1$. For $\lambda_{0}=0, \mathrm{Eq} \cdot(\mathrm{B} 8)$ gives

$$
\mathbf{M}_{0}(\tau, \lambda)=E_{1}^{(2)}(\tau)-\sum_{n}(-)^{n} \frac{\lambda^{n}}{n} E_{n+1}(\tau)
$$

while for $\lambda_{0}=1$, we obtain

$$
\mathrm{M}_{0}(\tau, \lambda)=\mathrm{M}_{0}(\tau)+\sum_{\mathrm{n}=1}(-)^{\mathrm{n}} \frac{(\lambda-1)^{\mathrm{n}}}{\mathrm{n}}\left[\mathrm{e}^{\tau} \sum_{\mathrm{i}=0}^{\mathrm{n}-1} 2^{-\mathrm{i}} \mathrm{E}_{\mathrm{i}+\mathrm{I}}(2 \tau)-\mathrm{E}_{\mathbf{l}}(\tau)\right] \text {. }
$$

In this last expression we have used the expansion

$$
\int_{1}^{\infty} \frac{d \mu}{\mu\left(\mu+\lambda_{0}\right)^{n}} e^{-\tau \mu}=\frac{1}{\lambda_{0}^{n}}\left[E_{1}(\tau)-\sum_{i=0}^{n} \lambda^{i} \int_{1}^{\infty} \frac{d \mu}{\left(\mu+\lambda_{0}\right)^{i+1}} e^{-\tau \mu}\right] .
$$

That the function $M_{0}(\tau, \lambda)$ is related to the integral

$$
\int_{1}^{\infty} \frac{\mathrm{d} \beta}{\beta+\alpha} \mathrm{E}_{1}[\mathrm{~h}(1+\beta)]=\mathrm{h} \int_{\mathrm{h}(1+\alpha)}^{\infty} \frac{\mathrm{d} \mu}{\mu} \mathrm{E}_{1}[\mu+\mathrm{h}(1-\alpha)]
$$

can be shown in the following manner. Consider the function.

$$
W_{1}(h x)=h x \int_{0}^{1} e^{-h x \mu} \ln \mu d \mu=x \int_{0}^{h} d y e^{-x y} \operatorname{lny}+\left(e^{-x h}-1\right) \operatorname{lnh} ;
$$

then

$$
x \int_{a}^{b} d y e^{-x y} \ln y=w_{1}(b x)-w_{1}(a x)-\left(e^{-x b}-1\right) \ln b+\left(e^{-x a}-1\right) \ln a .
$$

A further change of variables gives

$$
\int_{a-c}^{b-c} d \mu e^{-x} \mu_{l n}(\mu+c)=\frac{e^{x c}}{x}\left\{w_{1}(b x)-w_{1}(a x)-\left(e^{-x b}-1\right) \ln b+\left(e^{-x a}-1\right) \ln a\right\} .
$$


Integrating both sides of this expression over $\mathrm{x}$ from $\mathrm{xto} \mathrm{z}$, we obtain $\int_{a-c}^{b-c} d \mu \ln (\mu+c)\left\{\frac{e^{-x \mu}-e^{-z} \mu}{\mu}\right\}=\int_{x}^{z} d y \frac{e^{c y}}{y}\left\{w_{1}(b y)-w_{1}(a y)-\left(e^{-b y}-1\right) \ln b+\left(e^{-a y}-1\right) \ln a\right\}$.

In the limit as $\mathrm{b} \rightarrow \infty$, this becomes

$$
\int_{\delta}^{\infty} \frac{d \mu}{\mu} e^{-x \mu} \ln (\mu+c)=\int_{x}^{\infty} d y \frac{e^{c y}}{y}\left\{E_{1}[(\delta+c) y]+e^{-(\delta+c) y \ln (\delta+c)\}}\right.
$$

where $\delta=a-c$, and we have made use of the relation

$$
\mathrm{W}_{1}(\mathrm{x})=-\gamma-\ln |\mathrm{x}|-\mathrm{E}_{1}(\mathrm{x}) .
$$

Equation (B14) can be rewritten

$$
\int_{\delta}^{\infty} \frac{\mathrm{d} \mu}{\mu} \mathrm{e}^{-\mathrm{x} \mu \ell \mathrm{n}}(\mu+\mathrm{c})=\mathrm{E}_{\mathbf{1}}(\delta \mathrm{x}) \ln |\delta+\mathrm{c}|+\int_{\mathrm{x}}^{\infty} \mathrm{dy} \frac{\mathrm{e}^{\mathrm{cy}}}{\mathrm{y}} \mathrm{E}_{\mathbf{1}}[(\delta+\mathrm{c}) \mathrm{y}],
$$

where

$\int_{x}^{\infty} d y \frac{e^{c y}}{y} E_{1}[(\delta+c) y]=\int_{1}^{\infty} \frac{d \beta}{\beta} \int_{x}^{\infty} \frac{d y}{y} e^{[(\delta+c) \beta-c] y}=\int_{1}^{\infty} \frac{d \beta}{\beta} E_{1}[(\delta+c) x \beta-c x]$.

With $\delta=2, \mathrm{x}=\mathrm{h}$, and $\mathrm{c}=\alpha-1, \mathrm{Eq}(\mathrm{B} 15)$ becomes

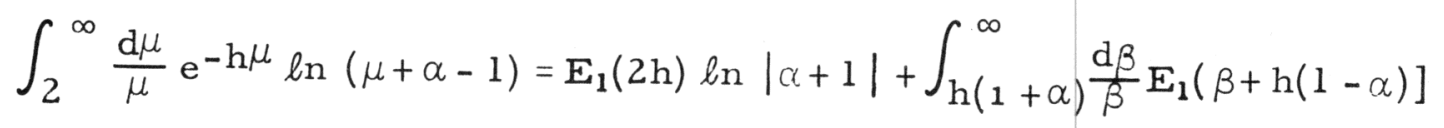

so that the integral of Eq. (Bll) is given by

$$
\int_{h(1+\alpha)}^{\infty} \frac{\mathrm{d} \mu}{\mu} \mathrm{E}_{1}[\mu+\mathrm{h}(1-\alpha)]=\mathrm{E}_{1}(2 \mathrm{~h}) \ln \frac{2}{|\alpha+1|}+\mathrm{M}_{0}\left(2 \mathrm{~h}, \frac{\alpha-1}{2}\right) .
$$

We shall use this relation in the evaluation of the integral $I_{S}^{(2)}, j+l(h, \alpha)$. 
AFPENDIX C

The Integral $\mathrm{I}_{\mathrm{s}, \mathrm{j}+1}^{(1)}(\mathrm{h}, \alpha)$

integral

In order to make the notation less cumbersome, consider the

$$
\mathrm{I}_{\ell \mathrm{m}}^{(1)}(\mathrm{h}, \alpha)=\int_{1}^{\infty} \frac{\mathrm{d} \gamma}{\gamma^{\ell}} \mathrm{e}^{-\gamma \mathrm{h}} \int_{0}^{\mathrm{h}} \mathrm{d} \tau \mathrm{e}^{(\gamma-\alpha) \tau} \mathrm{F}_{\mathrm{m}}(\tau, \alpha) .
$$

Although this involves a change in the meaning of the indices, no confusion should result. A recursion relation for this integral can be obtained by partial integration:

$$
\begin{aligned}
\mathrm{I}_{\ell \mathrm{m}}^{(1)}(\mathrm{h}, \alpha)= & \int_{0}^{\mathrm{h}} \mathrm{d} \tau \mathrm{E}_{\ell}(\mathrm{h}-\tau) \frac{\mathrm{d}}{\mathrm{d} \tau}\left\{\frac{\mathrm{F}_{\mathrm{m}}(\tau, 0)-\mathrm{e}^{-\alpha \tau} \mathrm{F}_{\mathrm{m}}(\tau, \alpha)}{\alpha}\right\} \\
= & \frac{\mathrm{E}_{\ell}(0)}{\alpha}\left\{\mathrm{F}_{\mathrm{m}}(\mathrm{h}, 0)-\mathrm{e}^{-\alpha \mathrm{h}} \mathrm{F}_{\mathrm{m}}(\mathrm{h}, \alpha)\right\} \\
& +\frac{1}{\alpha}\left\{\mathrm{I}_{\ell-1, \mathrm{~m}}^{(1)}(\mathrm{h}, \alpha)-\mathrm{I}_{\ell-1, \mathrm{~m}}^{(1)}(\mathrm{h}, 0)\right\}, \quad(l>1),
\end{aligned}
$$

where

$$
\begin{array}{r}
I_{\ell m}^{(1)}(h, 0)=E_{m+1}(0)\left[E_{\ell+1}(0)-E_{\ell+1}(h)\right]-G_{\ell, m+1}^{\prime}(h), \\
(\ell, m>0) .
\end{array}
$$

The integral

$$
\mathrm{G}_{\ell \mathrm{m}}^{\prime}(\tau)=\int_{0}^{\tau} \mathrm{dt} \mathrm{E}_{\mathrm{m}}(\mathrm{t}) \mathrm{E}_{\ell}(\tau-\mathrm{t})=\mathrm{G}_{\mathrm{m} \ell}^{\prime}(\tau)
$$

is discussed by Kourganoff and Chandrasekhar (op. cit.). Making use of the defining integrals for the $\mathrm{E}_{\ell}(\tau)$ and $\mathrm{F}_{\mathrm{j}}(\tau, \alpha)$-functions, Eq. (C1) can be rewritten

$$
\mathrm{I}_{\ell \mathrm{m}}^{(1)}(\mathrm{h}, \alpha)=\mathrm{e}^{-\alpha \mathrm{h}} \int_{0}^{\mathrm{h}} \mathrm{d} \tau \mathrm{e}^{\alpha \tau} \mathrm{G}_{\ell_{\mathrm{m}}}^{\prime}(\tau) .
$$

From Eq. (C4) it is obvious that

$$
\mathrm{I}_{\ell \mathrm{m}}^{(1)}(h, \alpha)=\mathrm{I}_{\mathrm{m} \ell}^{(1)}(\mathrm{h}, \alpha) \quad .
$$


Thus it is necessary to consider only the integral

$$
I_{1,1}^{(1)}(h, \alpha)=e^{-\alpha \cdot h} \int_{0}^{h} d \tau e^{\alpha T} G_{1,1}^{\prime}(\tau) .
$$

However it is convenient to treat the integral

$$
I_{1,1}^{(1)}(h, \epsilon, \alpha)=e^{-\alpha h} \int_{\epsilon}^{h} d \tau e^{\alpha \tau} G_{1,1}^{\prime}(\tau),
$$

and obtain Eq. (C7) by taking the limit as $\epsilon \rightarrow 0$. Making use of Eq. (K 37.30)*

$$
\begin{aligned}
I_{1,1}^{(1)}(h, \epsilon, \alpha) & =2 e^{-\alpha h} \int_{\epsilon}^{h} d \tau e^{\alpha \tau} \int_{1}^{\infty} d z \frac{e^{-\tau} z}{z} \int_{1}^{\infty} \alpha \frac{d x}{(x-z)} \\
& =-2 e^{-\alpha h} \int_{1}^{\infty} \frac{d z}{z^{2}} \frac{e^{h(\alpha-z)}-e^{\epsilon(\alpha-z)}}{\alpha-z} \ln (z-1) .
\end{aligned}
$$

Expanding the integrand in partial fractions and making use of Eq. (K 37.27) we obtain

$$
\begin{aligned}
\mathrm{I}_{1,1}^{(1)}(\mathrm{h}, \epsilon, \alpha)= & -\frac{2}{\alpha} \mathrm{N}_{1}(\mathrm{~h})+\frac{2}{\alpha} \mathrm{e}^{\alpha(\epsilon-\mathrm{h})} \mathrm{N}_{1}(\epsilon)-\frac{2}{\alpha^{2}} \mathrm{~N}_{0}(\mathrm{~h}) \\
& +\frac{2}{\alpha^{2}} \mathrm{e}^{\alpha(\epsilon-\mathrm{h})} \mathrm{N}_{0}(\epsilon)-\frac{2}{\alpha^{2}} \mathrm{e}^{-\alpha \mathrm{h}} \int_{\epsilon}^{\mathrm{h}} \mathrm{d} \tau \mathrm{e}^{\alpha \tau} \int_{1}^{\infty} \mathrm{dz} \mathrm{e}^{-\tau \mathrm{z}} \ln (\mathrm{z}-1) .
\end{aligned}
$$

This last integral can be written

$$
\int_{\epsilon}^{\mathrm{h}} \mathrm{d} \tau \mathrm{e}^{\alpha \tau} \int_{1}^{\infty} \mathrm{dz} \mathrm{e}^{-\tau \mathrm{z}} \ln (\mathrm{z}-1)=\int_{\epsilon}^{\mathrm{h}} \mathrm{d} \tau \mathrm{e}^{(\alpha-1) \tau} \int_{0}^{\infty} \mathrm{dy} \ln \mathrm{y} \mathrm{e}^{-\tau \mathrm{y}} ;
$$

*In the remainder of this Appendix references to specific equations in Kourganoff's Basic Methods in Transfer Problems will be denoted by $\mathrm{K}$ followed by the number of the equation as found in this text. 
the integral over $\mathrm{y}$ is just the Laplace transform of $l_{\mathrm{n}} \mathrm{y}$ which is

$$
-\frac{\gamma+\ell \mathrm{n} T}{T}
$$

Further, for $\alpha>1, \mathrm{Eq} .(\mathrm{C} 10)$ becomes

$$
\begin{aligned}
-\gamma \int_{\epsilon}^{\mathrm{h}} \mathrm{d} \tau \frac{\mathrm{e}^{(\alpha-1) \tau}}{\tau} & -\int_{\epsilon}^{\mathrm{h}} \mathrm{d} \tau \frac{\mathrm{e}^{(\alpha-1) \tau} \ln \tau}{\tau}=-\gamma \int_{\epsilon(\alpha-1)}^{\mathrm{h}(\alpha-1)} \mathrm{d} \mu \frac{\mathrm{e}^{\mu}}{\mu} \\
& -\int_{\epsilon(\alpha-1)}^{\mathrm{h}(\alpha-1)} \mathrm{du} \frac{\mathrm{e}^{\mu} \ln \mu}{\mu}+\ell \mathrm{n}(\alpha-1) \int_{\epsilon(\alpha-1)}^{\mathrm{h}(\alpha-1)} \mathrm{d} \mu \frac{\mathrm{e}^{\mu}}{\mu} .
\end{aligned}
$$

These integrals are directly related to the $E_{1}(x)$ and $E_{1}^{(2)}(x)$ integrals for negative arguments. Integrating by parts, it can be shown that

$$
\int_{\delta}^{\mathrm{a}} \mathrm{d} \mu \frac{\mathrm{e}^{\mu} \ln \mu}{\mu}=-\mathrm{E}_{1}(-\mathrm{a}) \ln \mathrm{a}+\mathrm{E}_{1}(-\delta) \ln \delta+\mathrm{E}_{1}^{(2)}(-\delta)-\mathrm{E}_{1}^{(2)}(-\mathrm{a})
$$

Combining these results and taking the limit as $\epsilon \rightarrow 0$, we obtain for $\alpha>1$

$$
\begin{aligned}
I_{1,1}^{(1)}(h, \alpha)= & -\frac{2}{\alpha} N_{1}(h)+\frac{2}{\alpha^{2}} E_{1}^{(2)}(h)+\frac{2}{\alpha^{2}}(\gamma+l n h) E_{1}(h) \\
+ & \frac{2 e^{-\alpha h}}{\alpha^{2}}\left\{[-\gamma-l n h] E_{1}(-h(\alpha-1))-E_{1}^{(2)}(-h(\alpha-1))\right. \\
& \left.+\frac{1}{2} l n^{2}(\alpha-1)\right\}
\end{aligned}
$$




\section{APPENDIX D}

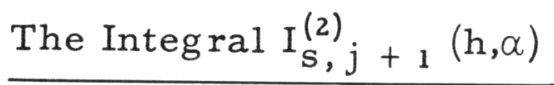

Again with a change in the meaning of the indices, we rewrite Eq. (73) in the form

$$
\begin{aligned}
\operatorname{Ilm}_{l \mathrm{~m}}^{(2)}(\mathrm{h}, \alpha) & =\int_{0}^{\mathrm{h}} \mathrm{d} \tau \int_{1}^{\infty} \frac{\mathrm{d} \gamma}{\gamma l} \mathrm{e}^{(\gamma-\alpha) \tau} \mathrm{e}^{-\gamma \mathrm{h}} \mathrm{F}_{\mathrm{m}}(\mathrm{h}-\tau,-\alpha) \\
& =\mathrm{e}^{-\alpha \mathrm{h}} \int_{1}^{\infty} \frac{\mathrm{d} \gamma}{\gamma l} \int_{0}^{\mathrm{h}} \mathrm{d} \tau \mathrm{e}^{(\alpha-\gamma) \tau} \mathrm{F}_{\mathrm{m}}(\tau,-\alpha) \\
& =\mathrm{e}^{-\alpha \mathrm{h}} \int_{0}^{\mathrm{h}} \mathrm{d} \tau \mathrm{e}^{\alpha \tau} \mathrm{E}_{\ell}(\tau) \mathrm{F}_{\mathrm{m}}(\tau,-\alpha) .
\end{aligned}
$$

As in Appendix C recursion formulae can be obtained for this integral by partial integration:

$$
\begin{aligned}
I_{l m}^{(2)}(h, \alpha) & =e^{-\alpha h} \int_{0}^{h} \mathrm{~d} \tau F_{m}(\tau,-\alpha) \frac{d}{d \tau} F_{l}(\tau, \alpha) \\
& =e^{-\alpha h} F_{m}(h,-\alpha) F_{l}(h, \alpha)-I_{m_{l} l}^{(2)}(h,-\alpha),
\end{aligned}
$$

and

$$
\begin{aligned}
& I_{l m}^{(2)}(h, \alpha)=e^{-\alpha h} \int_{0}^{h} \mathrm{~d} \tau E_{l}(\tau) \frac{d}{d \tau}\left\{\frac{e^{\alpha \tau} F_{m}(\tau,-\alpha)-F_{m}(\tau, 0)}{\alpha}\right\} \\
& =\frac{E_{\ell}(h)}{\alpha}\left\{F_{m}(h,-\alpha)-e^{-\alpha h} F_{m}(h, 0)\right\}+\frac{1}{\alpha}\left\{I_{l}^{(2)}-1, m(h, \alpha)-e^{-\alpha h} I_{l-1, m}^{(2)}(h, 0)\right\}
\end{aligned}
$$

where

$$
(\ell>1)
$$

$$
I_{l m}^{(2)}(0)=E_{m+1}(0)\left\{E_{l+1}(0)-E_{l+1}(h)\right\}-G_{l, m+1}(h) .
$$

The integral

$\mathrm{G}_{\ell, \mathrm{m}+1}(\mathrm{~h})=\int_{0}^{\mathrm{h}} \mathrm{d} \tau \mathrm{E}_{\ell}(\tau) \mathrm{E}_{\mathrm{m}+{ }_{1}}(\tau)=\frac{\mathrm{h} \mathrm{E}_{\ell}(\mathrm{h}) \mathrm{E}_{\mathrm{m}+1}(\mathrm{~h})+\mathrm{F}_{\ell}(\mathrm{h},-1)+\mathrm{F}_{\mathrm{m}+1}(\mathrm{~h},-1)}{\ell+\mathrm{m}}$ 
is discussed by Chandrasekhar.*

It is necessary therefore to investigate only $I_{1, m}^{(2)}(h, \alpha)$, where

$$
\mathrm{I}_{1 \mathrm{~m}}^{(2)}(\mathrm{h}, \alpha)=\mathrm{e}^{-\alpha \mathrm{h}} \int_{0}^{\mathrm{h}} \mathrm{d} \tau \mathrm{e}^{\alpha \tau} \mathrm{E}_{1}(\tau) \mathrm{F}_{\mathrm{m}}(\tau,-\alpha) .
$$

Making use of the recursion relation (B2), this can be rewritten

$$
I_{1 m}^{(2)}(h, \alpha)=\frac{e^{-\alpha h}}{\alpha}\left\{\frac{1}{m-1} F_{1}(h, \alpha)-G_{m, 1}(h)\right\}-\frac{1}{\alpha} I_{1, m-1}^{(2)}(h, \alpha),(m>1) .
$$

The recursion relations (D3) and (D7) permit the evaluation of any integral, $I_{\mathrm{l}}^{(2)}(h, \alpha)$, in terms of

$$
I_{1}^{(2), 1}(h, \alpha)=e^{-\alpha h} \int_{0}^{h} d \tau e^{\alpha \tau} E_{1}(\tau) F_{1}(\tau,-\alpha)
$$

Making use of the definition of $F_{1}$, this becomes

$I_{1,1}^{(2)}(h, \alpha)=e^{-\alpha h}\left\{\frac{1}{\alpha} l n|\alpha+1| F_{1}(h, \alpha)-\frac{1}{\alpha} \int_{0}^{h} d \tau\left[E_{1}(\tau)\right]^{2}+\frac{1}{\alpha} \int_{0}^{h} d \tau E_{1}(\tau) \int_{1}^{\infty} d \beta \frac{e^{-\beta \tau}}{\beta+\alpha}\right\}$

where**

$$
\int_{0}^{h} \mathrm{~d} \tau\left[E_{1}(\tau)\right]^{2}=2 \ln 2-E_{2}(h) E_{1}(h)-e^{-h} E_{1}(h)+2 E_{1}(2 h)
$$

Next consider

$$
\begin{aligned}
\int_{0}^{h} \mathrm{~d} \tau \mathrm{E}_{1}(\tau) \int_{1}^{\infty} \mathrm{d} \beta \frac{\mathrm{e}^{-\beta \tau}}{\beta+\alpha}= & -\mathrm{E}_{1}(\mathrm{~h}) \frac{\mathrm{E}_{1}(\mathrm{~h})-\mathrm{e}^{-\alpha \mathrm{h}} \mathrm{E}_{1}[(1+\alpha) \mathrm{h}]}{\alpha} \\
& +\int_{1}^{\infty} \frac{\mathrm{d} \beta}{\beta(\beta+\alpha)} \ln (1+\beta)+\int_{1}^{\infty} \frac{\mathrm{d} \beta}{\beta(\beta+\alpha)} \mathrm{E}_{1}[\mathrm{~h}(1+\beta)]
\end{aligned}
$$

*Chandrasekhar, Radiative Transfer, Oxford (1950), p. 376.

**J. Le Caine, A Table of Integrals Involving the Functions $E_{n}(x)$,

N.R.C. No. 1553; Eqs. (3.2.1) and (3.3.1). 
where

$$
\int_{1}^{\infty} \frac{d \beta}{\beta(\beta+\alpha)} \ln (1+\beta)=\left\{\begin{array}{l}
\frac{1}{\alpha}\left\{\frac{\pi^{2}}{12}+\sum_{n=1}^{\infty} \frac{(-)^{n}}{n^{2}}\left(\frac{1-\alpha}{1+\alpha}\right)^{n}+\frac{\ln ^{2}(1+\alpha)}{2}\right\}, \quad(\alpha>0) \\
-\frac{1}{\alpha}\left\{\frac{\pi^{2}}{12}+\sum_{n=1}^{\infty} \frac{(-1)^{n}}{n^{2}}\left(\frac{1+\alpha}{1-\alpha}\right)^{n}+\frac{\ln ^{2}(1-\alpha)}{2}-\ln (1-\alpha) \ln (1+\alpha)\right\},(-1<\alpha \leqslant 0) \quad .
\end{array}\right.
$$

(Le Caine, Eqs. (B.1.2) and (B.3.1). In view of this limitation on negative values of $\alpha$, it may be necessary to evaluate the set of integrals, $I^{2}, m(h, \alpha)$, for positive $\alpha$ and use Eq. (D2) to obtain the corresponding set for negative values of $\alpha$. Finally

$\int_{1}^{\infty} \frac{\mathrm{d} \beta}{\beta(\beta+\alpha)} \mathrm{E}_{1}[\mathrm{~h}(1+\beta)]=\frac{1}{2 \alpha}\left[\mathrm{E}_{1}(\mathrm{~h})\right]^{2}-\frac{1}{\alpha}\left[\mathrm{hE}_{1}(2 \mathrm{~h}) \ln \frac{2}{|\alpha+1|}+\mathrm{h} \mathrm{M}_{0}\left(2 \mathrm{~h}, \frac{\alpha-1}{2}\right)\right]$

where we have used Eq. (B17).

Combining these results, we have for $\alpha \geqslant 0$

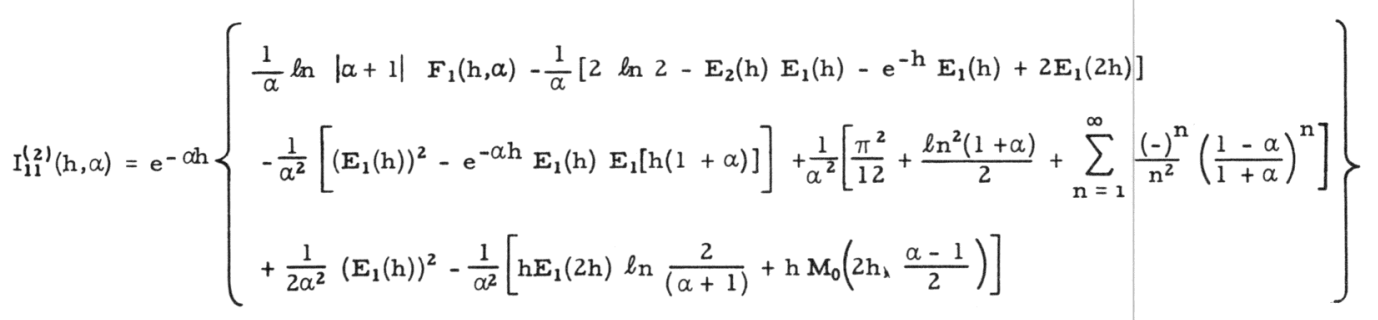




\section{APPENDIX E}

Summary of the Newer Data Published in the Annals of Physics*

We present here in graphical form the results of an analysis of the angular distributions obtained in the scattering of neutrons from various nuclei. These measurements are a continuation of the survey of neutron angular distributions described previously, except that the data presented here were obtained from measurements at eight scattering angles rather than at five as in the previous work. The results include a series of measurements of the neutrons scattered from Li ${ }^{6}(96 \%)$, Li (normal), Be, $\mathrm{C}, \mathrm{Si}, \mathrm{Ca}, \mathrm{Cu}, \mathrm{Sn}, \mathrm{Pb}$, and $\mathrm{U}^{238}$ in the energy range from 50 kev to $2300 \mathrm{kev}$. The energy spread of the incident neutron beam was sufficient to average out most of the pronounced fluctuations due to individual scattering resonances. In addition, a series of angular distributions of neutrons scattered at resonances was measured for $\mathrm{C}^{12}, \mathrm{O}^{16}, \mathrm{Si}, \mathrm{S}^{32}, \mathrm{Li}^{6}$, and $\mathrm{Li}^{7}$. At present it will suffice to say that the neutrons used for this study were obtained from the proton bombardment of evaporated lithium targets in the $\mathrm{Li}^{7}(\mathrm{p}, \mathrm{n}) \mathrm{Be}^{7}$ reaction. The neutrons passed down a collimator and were incident on scattering samples in the form of flat slabs placed at the center of a circular track. The scattered neutrons were detected by oil-moderated $\mathrm{BF}_{3}$ proportional counters in specially constructed shield tanks, ** which could be moved independently along the track. There were five such detector tanks and, except for limitations due to their size, any combination of angles between $22^{\circ}$ and $145^{\circ}$ could be obtained. The forward neutron flux was monitored by two additional oil-moderated $\mathrm{BF}_{3}$ detectors.

For the measurements with broad resolution, the lithium targets produced neutrons with an energy spread of about $40 \mathrm{kev}$ over the range of neutron energies between 50 and $600 \mathrm{kev}$, and neutrons with spreads of 80 to $130 \mathrm{kev}$ in the range from 600 to $2250 \mathrm{kev}$. The scattering samples were chosen to give approximately $90 \%$ transmission. The $\mathrm{Li}, \mathrm{Ca}$, and Si samples were "canned" in thin-walled steel containers. For the measurements of resonance scattering, the total spread in the incident neutron beam was limited to between 5 and $10 \mathrm{kev}$. In the $\mathrm{O}^{16}$ measurements, "canned" samples of $\mathrm{SnO}_{2}$ and matching $\mathrm{Sn}$ samples were employed.

Analysis

The data are presented in terms of the coefficients $B_{\ell}$ in the expansion of the differential scattering cross section $\sigma_{S}(\mu)$ in a series of

*R. O. Lane, A. Langsdorf, Jr。, E. Monahan, and A。J.Elwyn, Ann. Phys.12, 135-171 (1961)。 This summary is from Argonne National Laboratory Report ANL-6169 (June 1960), pp.4-22。

**Alexander Langsdorf, Jr。, Fast Neutron Physics, Part I, edited by J.B. Marion and J.L.Fowler (Interscience Publishers, New York, 1960), p. 721 . 
Legendre polynomials $\mathrm{P}_{\ell}(\mu)$ of the form

$$
\sigma_{\mathbf{s}}(\mu)=\sum_{l}^{\mathrm{N}-1} \mathrm{~B}_{\ell} \mathrm{P}_{\ell}(\mu),
$$

where $\mu=\cos \theta$, and $\theta$ is the scattering angle. The values of the coefficients $\mathrm{B}_{\ell}$ were obtained from an analysis of the entire set of data by means of a least-squares procedure.

In order to obtain the proper relation between the normalized yields and the differential scattering cross section, it was necessary to make a number of corrections to the data and to determine the absolute efficiency factor for the experimental setup. Corrections for the attenuation of the primary neutron beam and for the finite resolution of the experimental equipment were made in essentially the same manner as previously described. Multiple scattering of the neutrons was taken into account on the basis of an energy-dependent calculation by Monte Carlo procedures described in the Annals article and by Lane and Miller .* Further corrections, to take into account the variations in detector efficiency as a function of neutron energy and the effects of the second group of neutrons in the $\mathrm{Li}^{7}(\mathrm{p}, \mathrm{n}) \mathrm{Be}^{7}$ reaction (arising from a state in $\mathrm{Be}^{7}$ at $0.43 \mathrm{Mev}$ excitation), were made to the data in those cases in which the effects were considered significant.

The absolute efficiency factor was evaluated by normalizing all integrated (with respect to angle) scattering cross sections to the independently measured scattering cross section of some standard scatterer. For most of this work, the standard sample chosen was carbon.

$\underline{\text { Results }}$

Figures 1 through 15 present the results of the angular-distribution measurements and analysis. For each scattering sample, the coefficients $\mathrm{B}_{\ell}$ are given as functions of the incident energy $\mathrm{E}$. Both $\mathrm{B}_{\ell}$ and $\mathrm{E}$ are given in the laboratory system of coordinates. The value of $E$ used in this work is calculated as

$$
\mathrm{E}=\mathrm{E}_{\mathrm{m}}-\frac{1}{2} \Delta
$$

where $E_{m}$ is the maximum energy of the neutrons in the incident beam and $\Delta$ is the half width of the energy distribution of the neutron beam (as determined from "rise curve" measurements just above threshold). Also shown on the figures are the differential cross sections as a function of $\cos \theta$ in the laboratory system at a number of incident energies $\mathrm{E}$.

*R. Lane and W.F. Miller, "The Multiple Scattering Effects of Neutrons in Thin Plates" (to be published). 
Both the $\mathrm{B}_{\ell}$ and the differential cross sections are given in barns per steradian. The solid curve through the points on the plots of $B_{l}$ are drawn merely to indicate the trend of the data, whereas those on the plots of differential cross sections are the results of the least-squares fitting. It is to be noted that $4 \pi \mathrm{B}_{0}$ is equivalent to the total scattering cross section for the element under consideration. The numerical values for the coefficients and differential cross sections are tabulated in a separate report.*

The error bars drawn on the figures represent the standard deviation associated with the $\mathrm{B}_{\ell}$. Included in such an error is the error in the total cross section of the standard scatterer, i.e., carbon. In most cases, this error gave the dominant contribution to the errors shown.

A comparison of the results of these measurements with the original five-angle data, for those cases in which overlap exists, showed no differences greater than those expected on the basis of the assigned errors, except in those cases in which the $\mathrm{B}_{\ell}$ for $l>4$ were significantly nonzero.

* These detailed results are tabulated in an informal report entitled "Tables of Differential Cross Sections for Neutrons Scattered from Various Nuclei," by R. O. Lane, A.S. Langsdorf, Jr., J。E. Monahan, and A.J. Elwyn. This report may be obtained from the authors. 


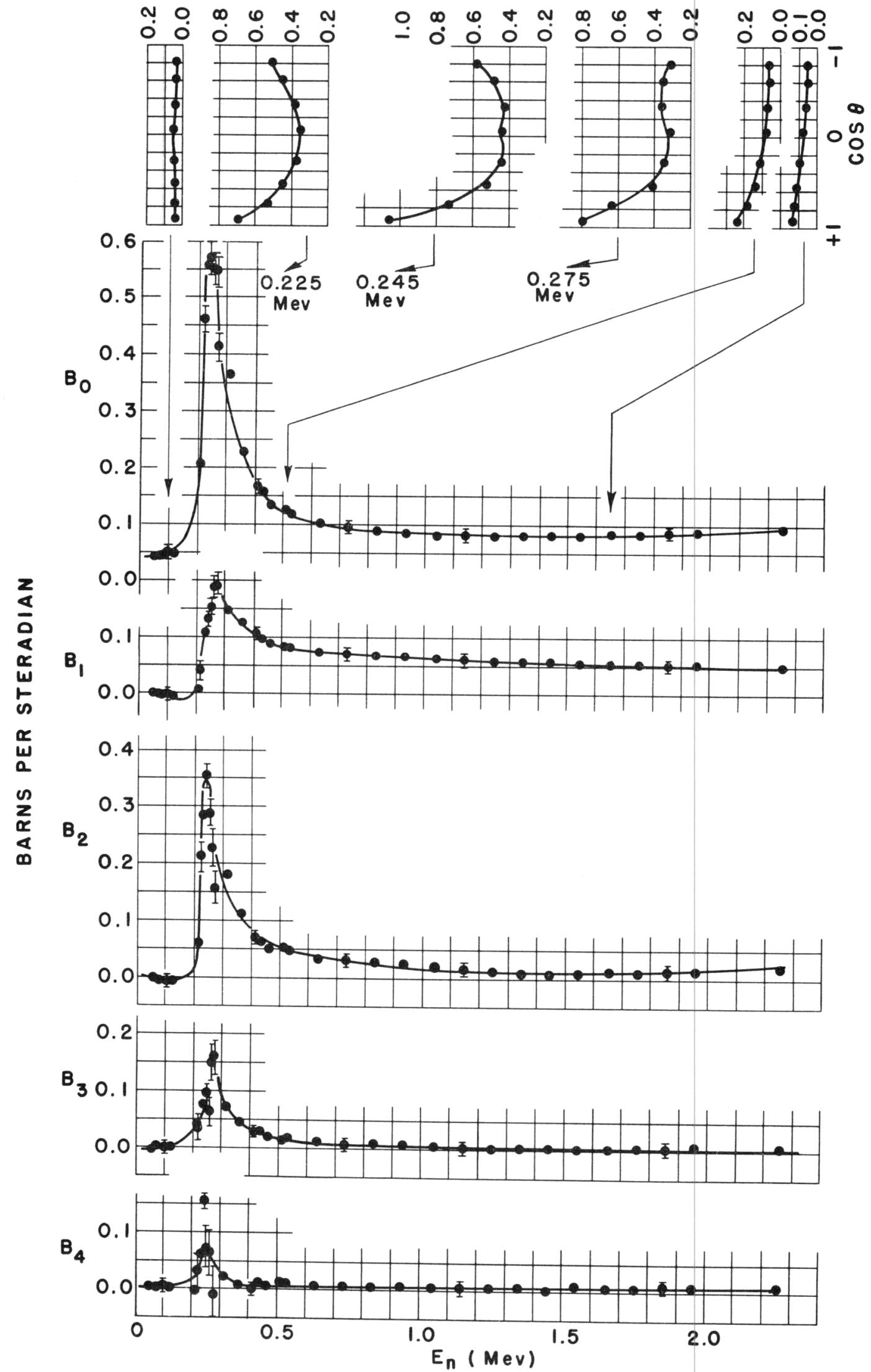

Fig. 1. The differential cross section for scattering of neutrons by $\mathrm{Li}^{6}$. 

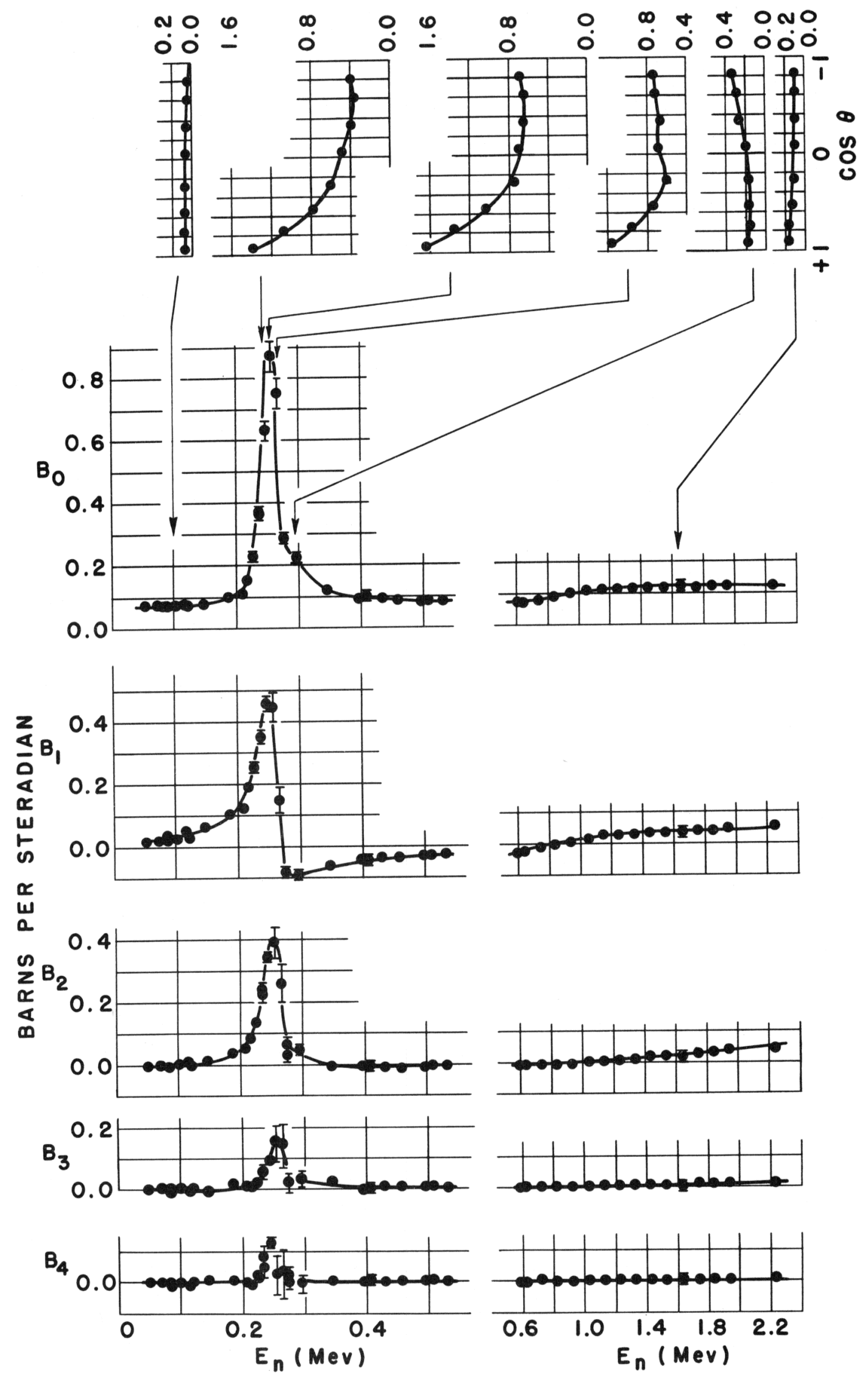

Fig. 2. The differential cross section for scattering of neutrons by $\mathrm{Li}^{7}$. 

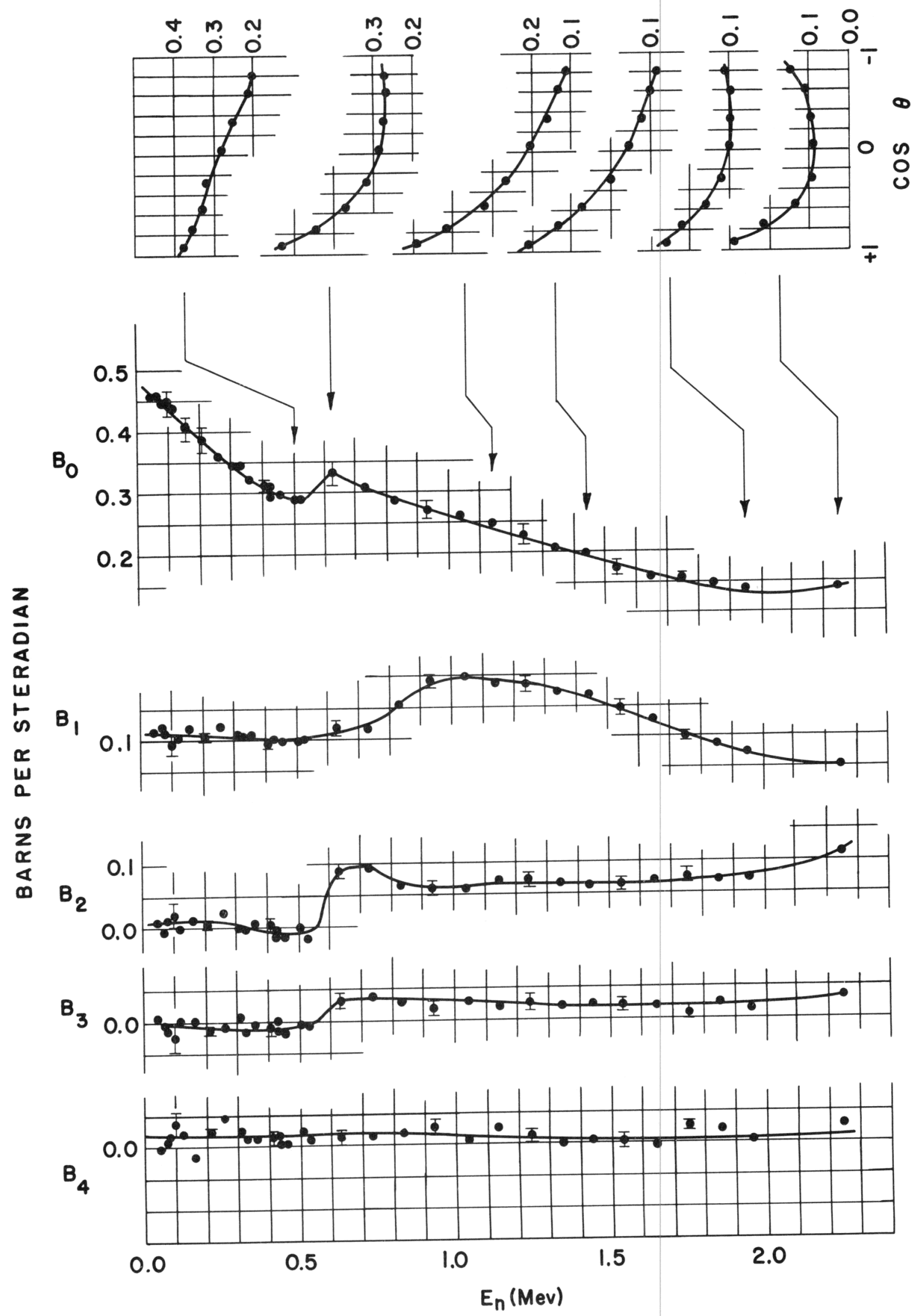

Fig. 3. The differential cross section for scattering of neutrons by Be. 


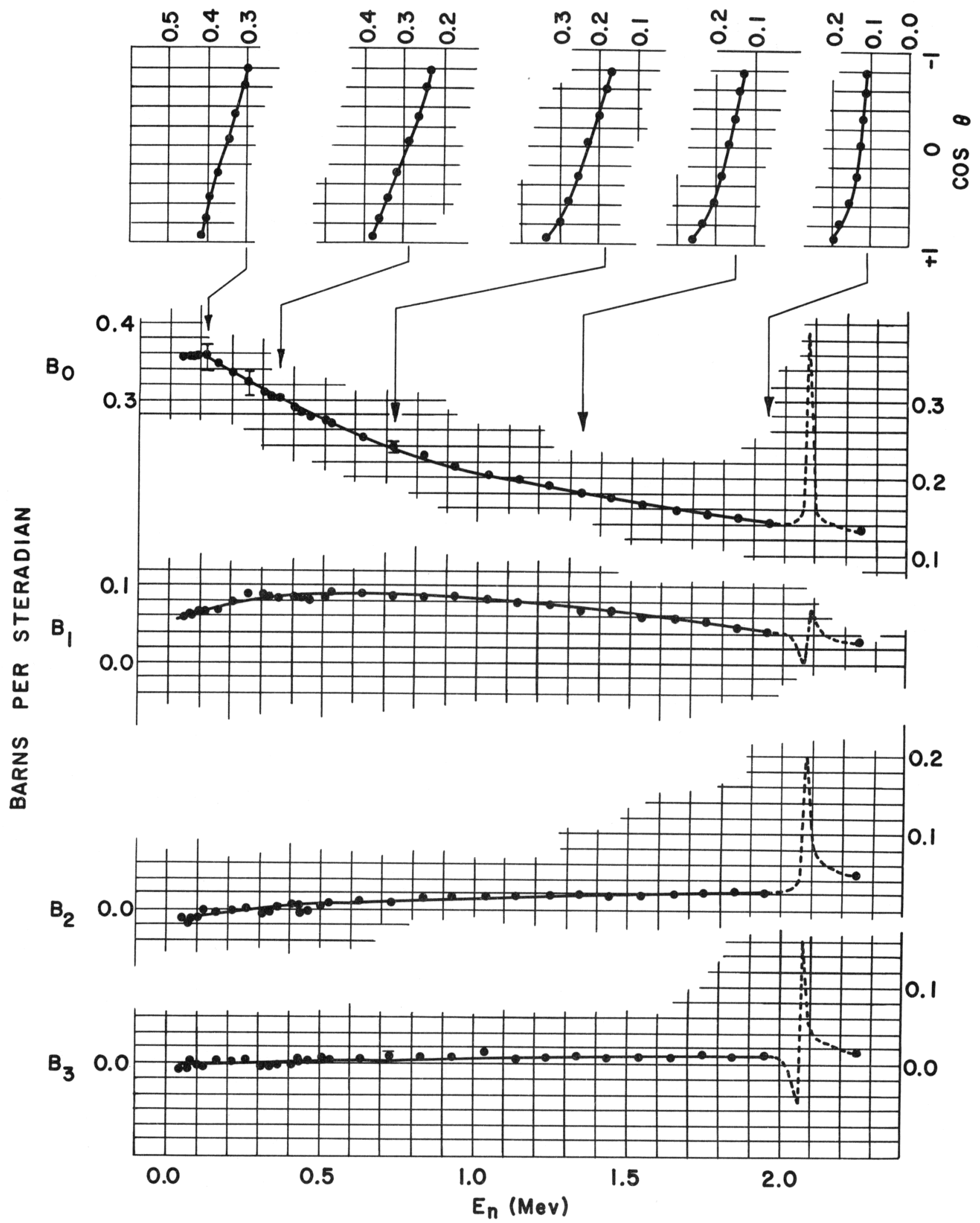

Fig. 4. The differential cross section for scattering of neutrons by $\mathrm{C}^{12}$. 

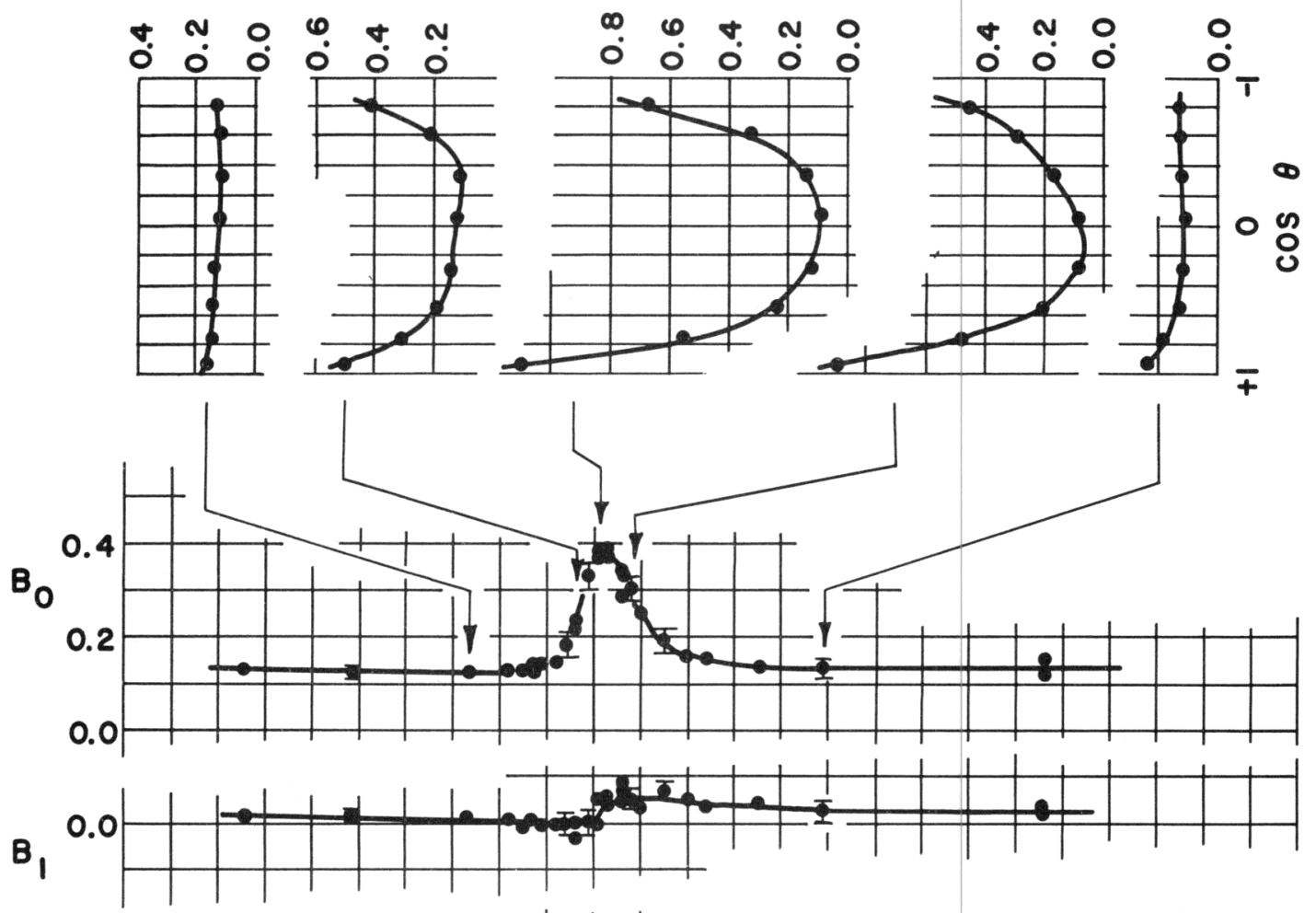

$\frac{2}{0}$

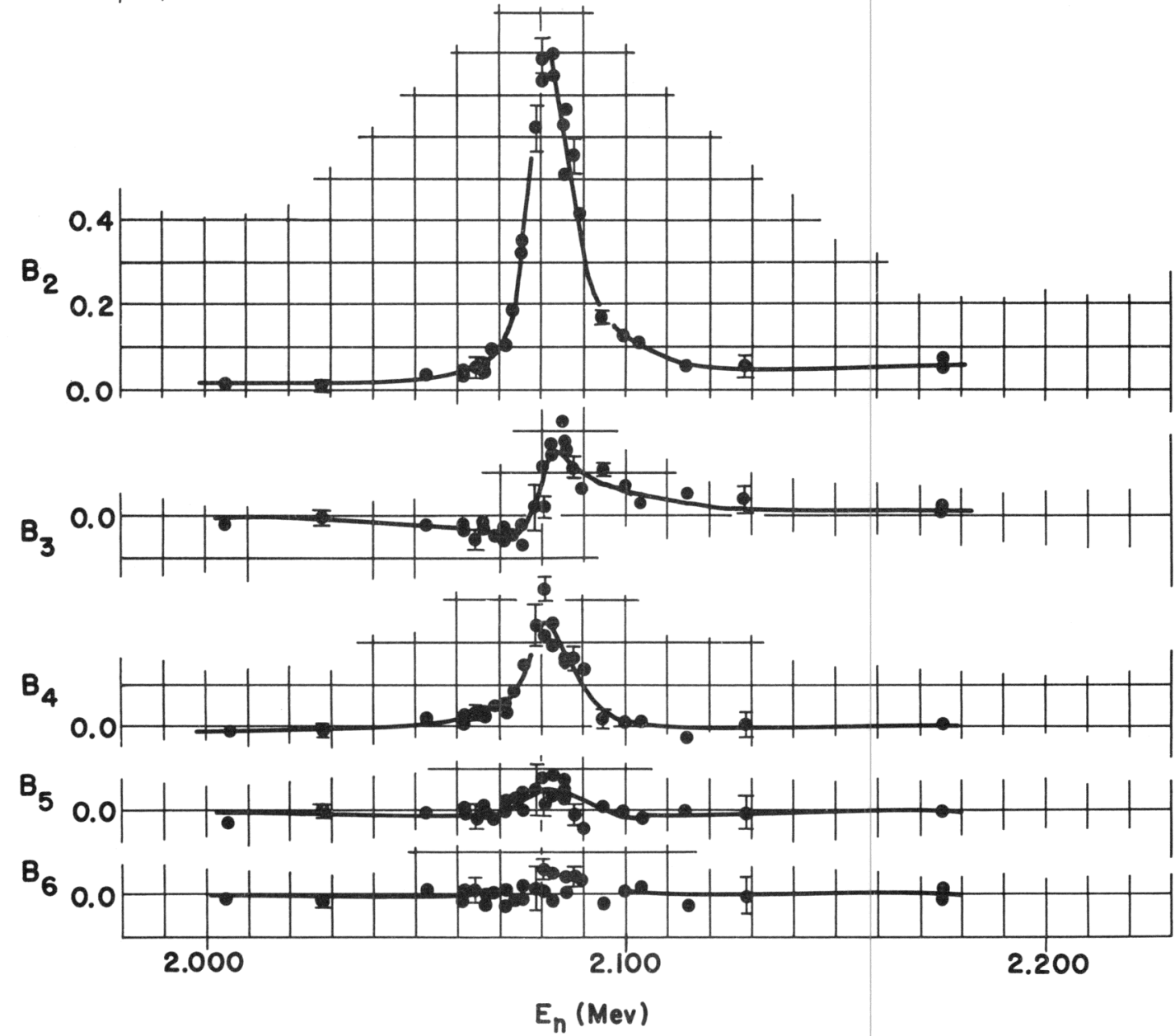

Fig. 5. The differential cross section for scattering of neutrons by $\mathrm{C}^{\mathbf{1 2}}$. 


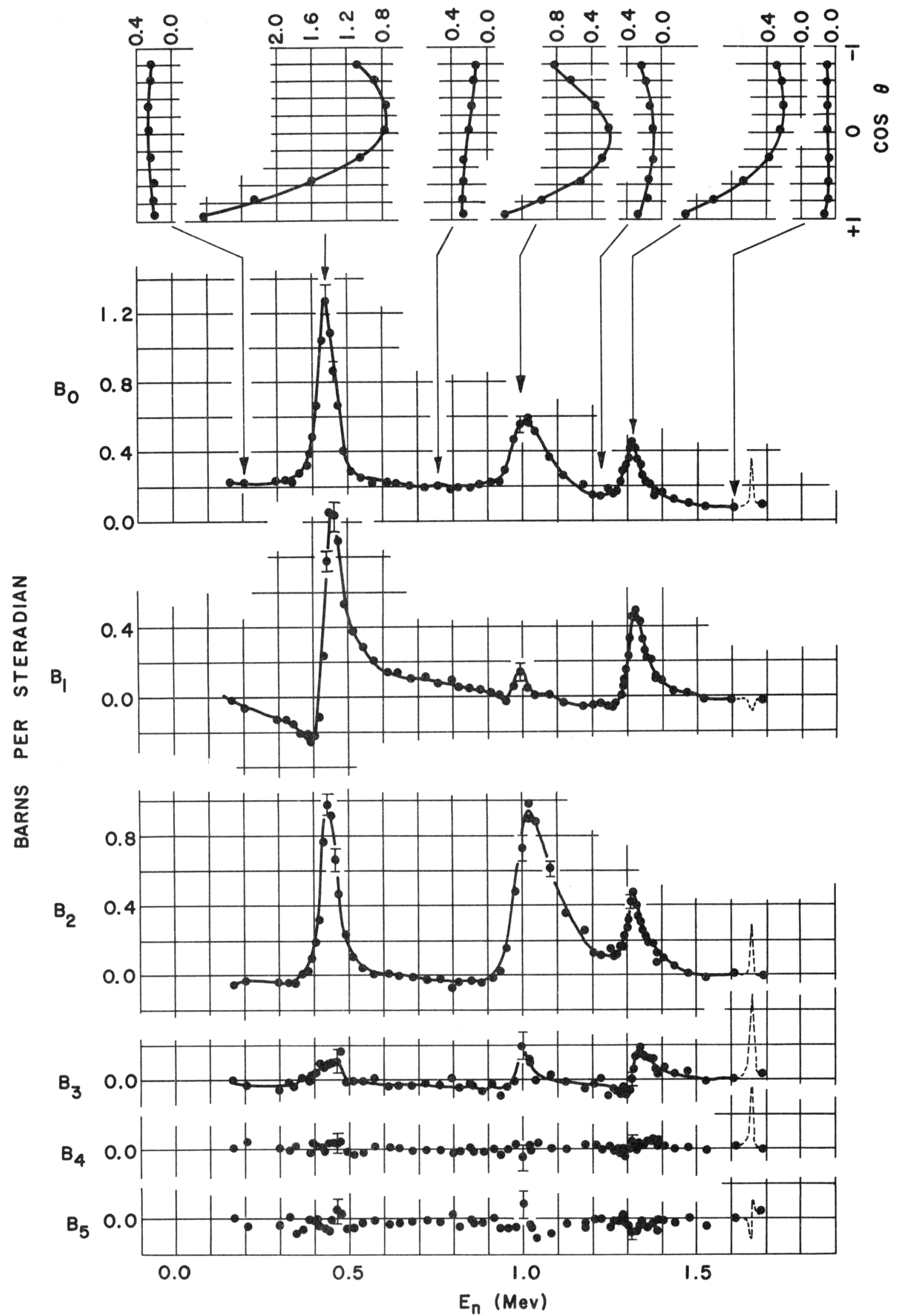

Fig. 6. The differential cross section for scattering of neutrons by $\mathrm{O}^{16}$. 


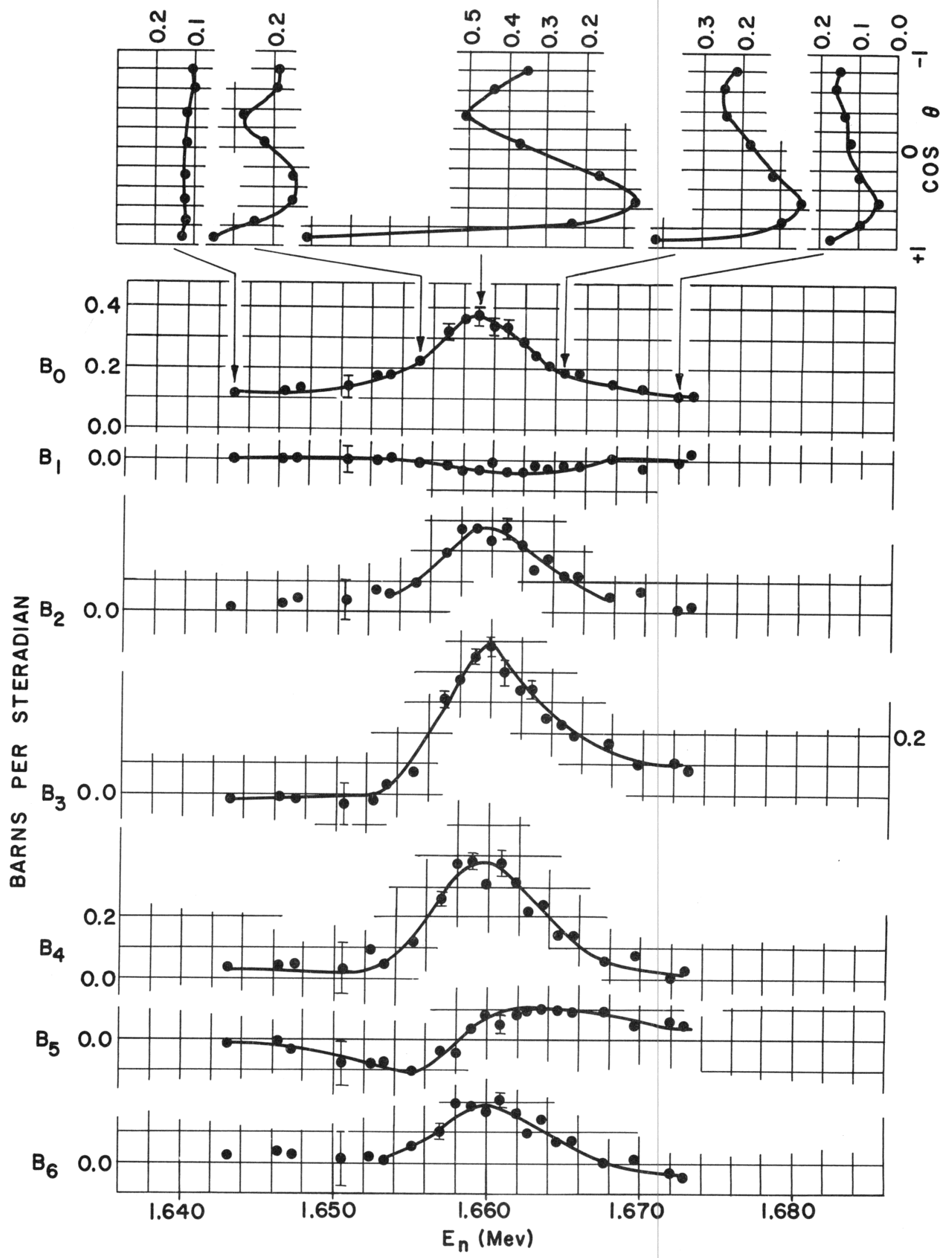

Fig. 7. The differential cross section for scattering of neutrons by $\mathrm{O}^{16}$. 


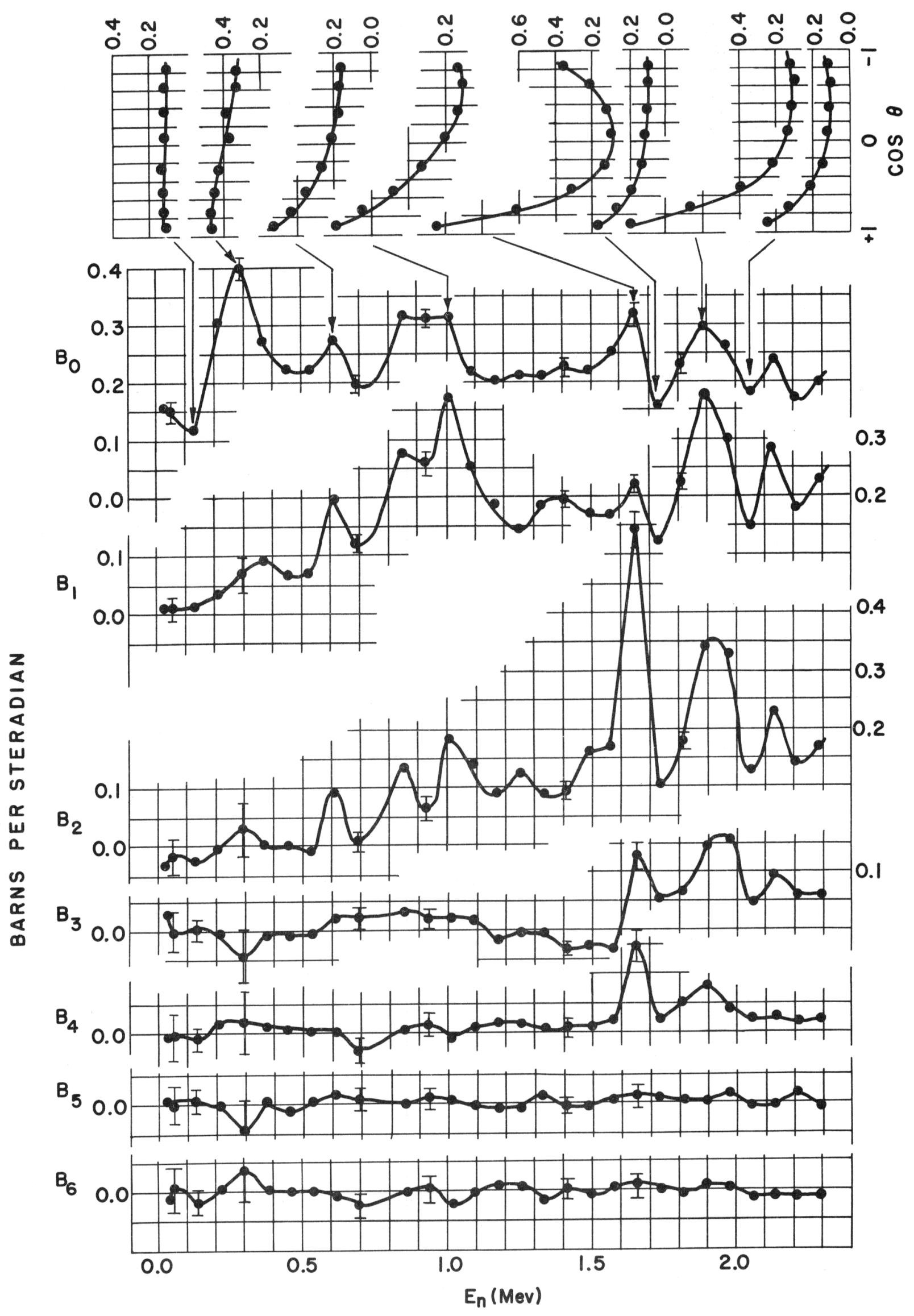

Fig. 8. The differential cross section for scattering of neutrons by Si. 


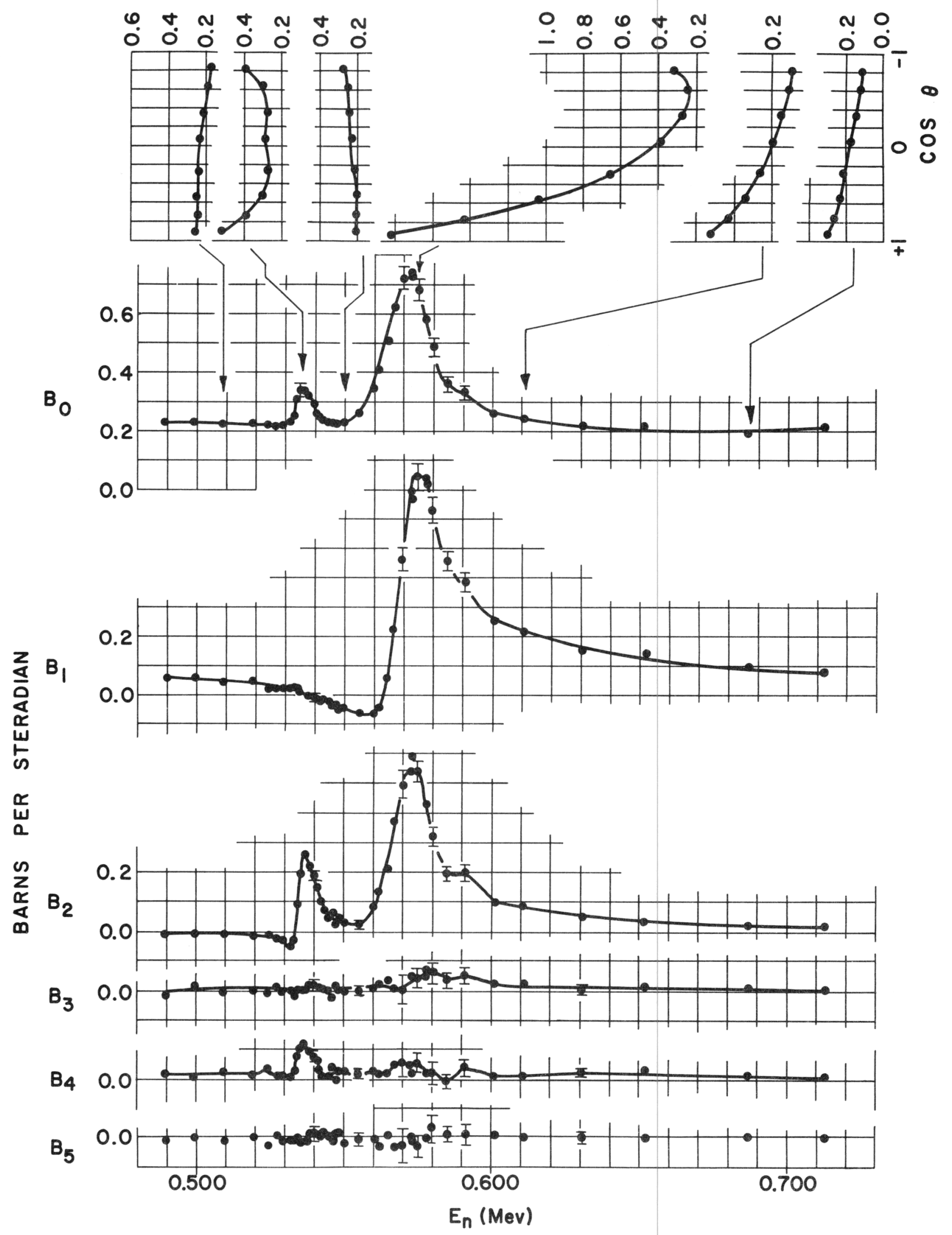

Fig. 9. The differential cross section for scattering of neutrons by Si. 


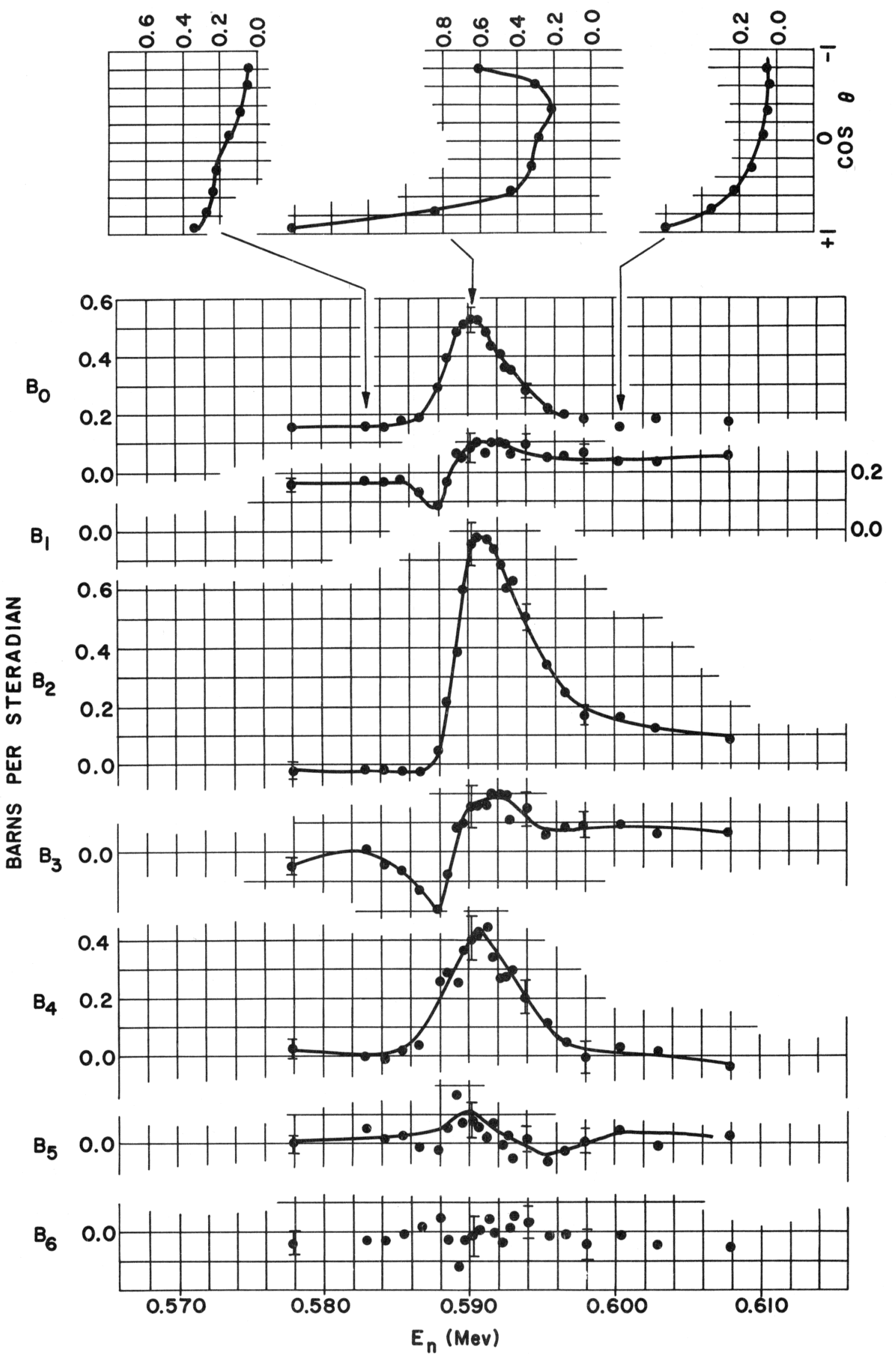

Fig. 10. The differential cross section for scattering of neutrons by S. 

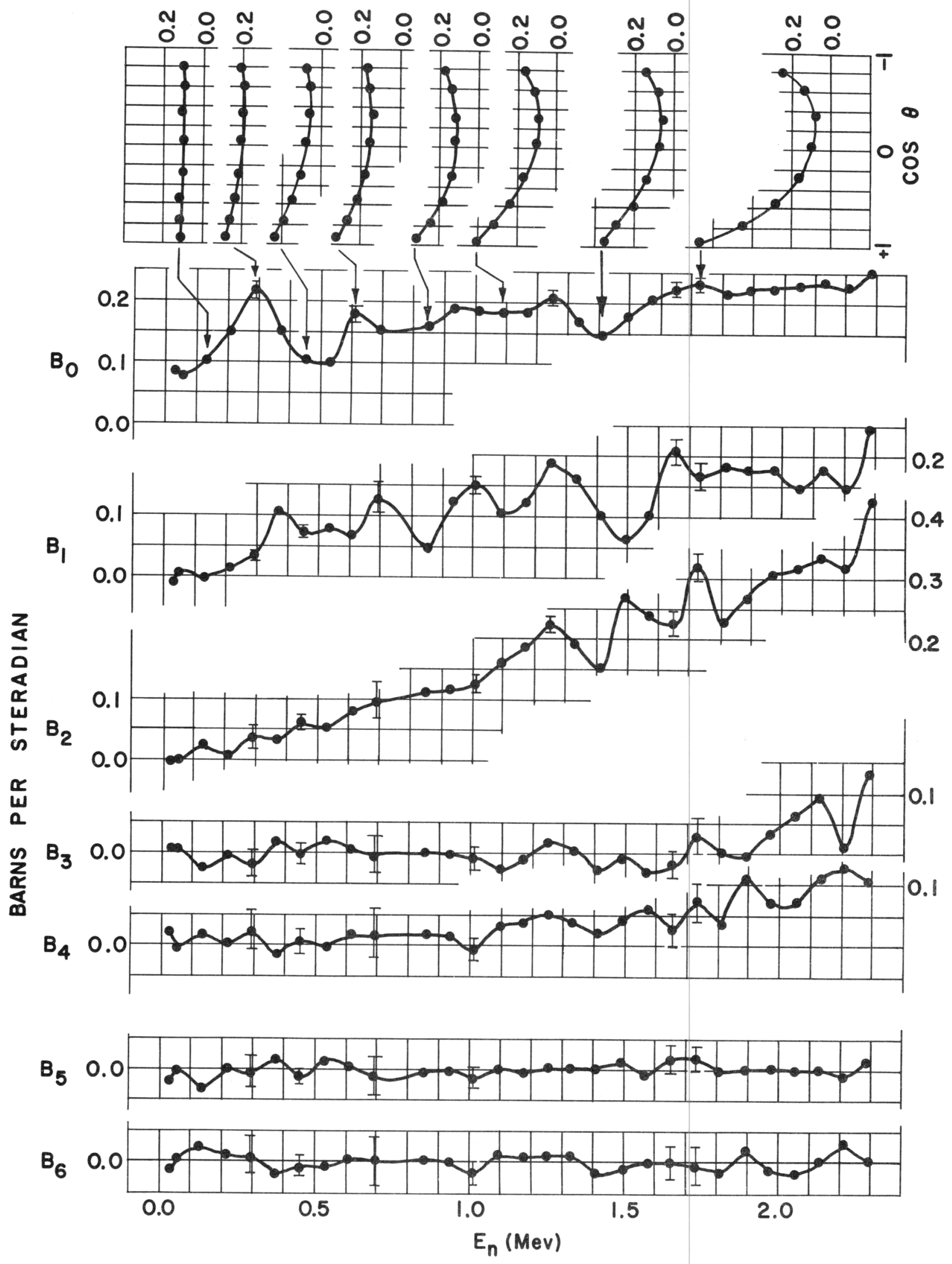

Fig. 11. The differential cross section for scattering of neutrons by Ca. 


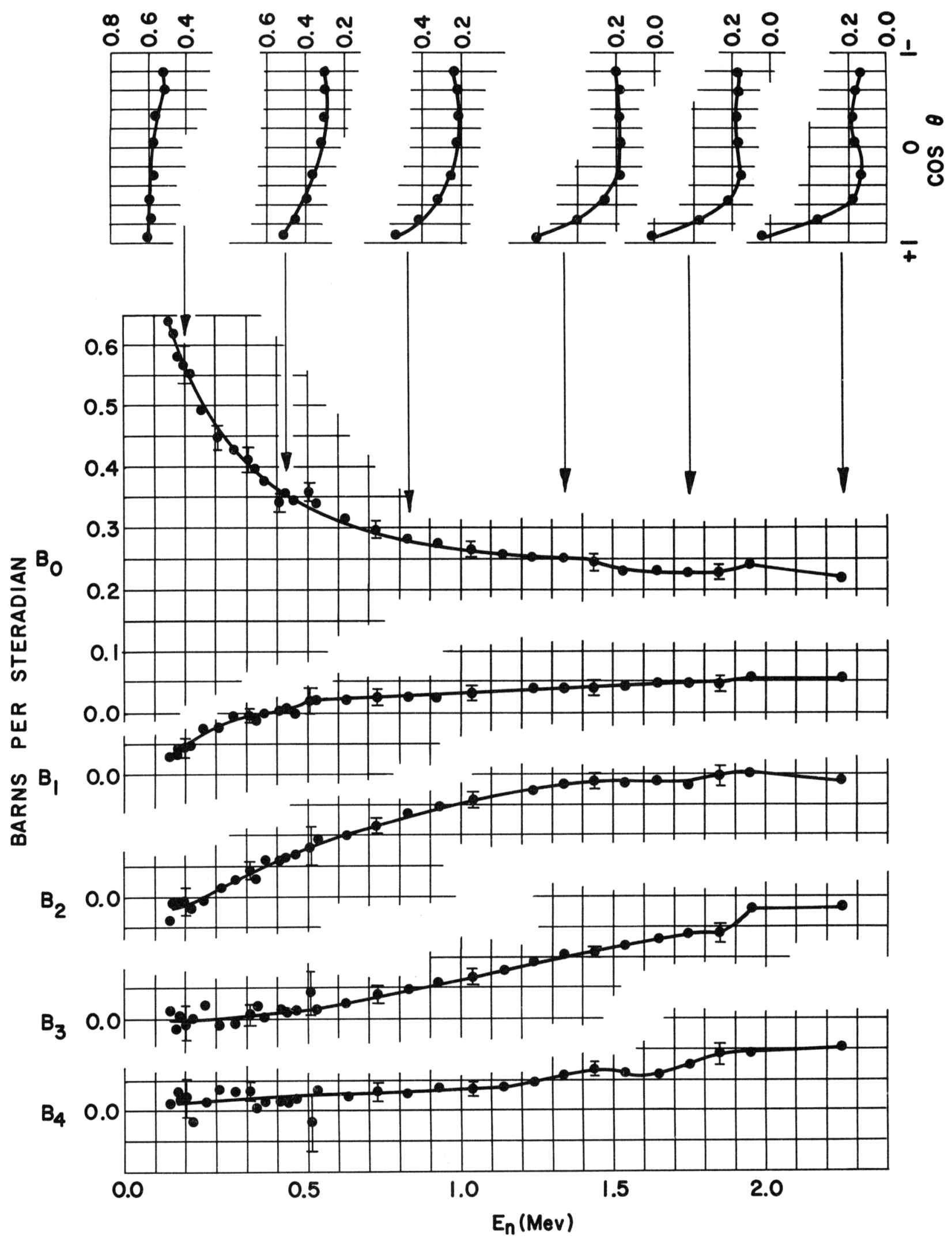

Fig. 12. The differential cross section for scattering of neutrons by $\mathrm{Cu}$. 


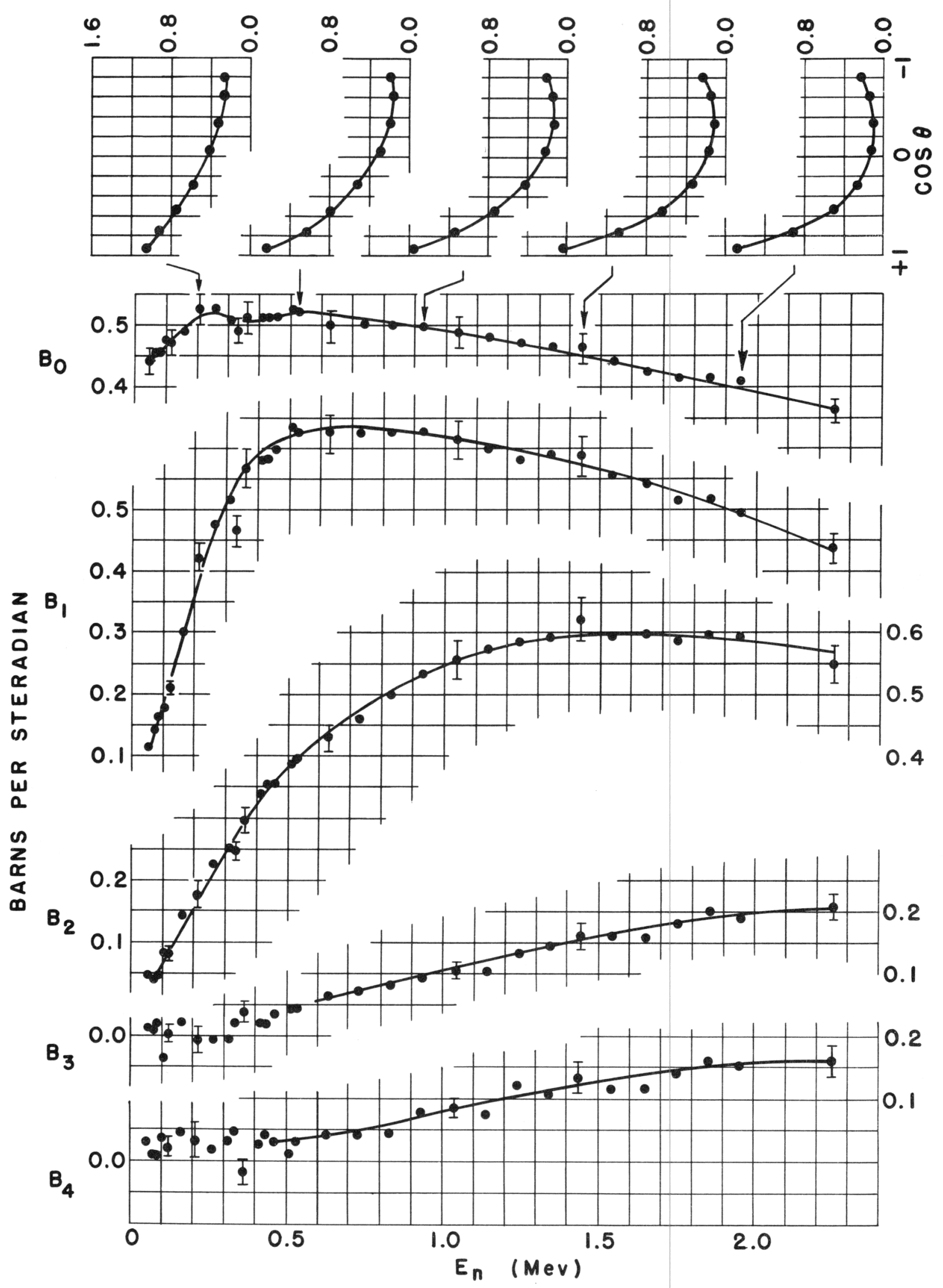

Fig. 13. The differential cross section for scattering of neutrons by $\mathrm{Sn}$. 


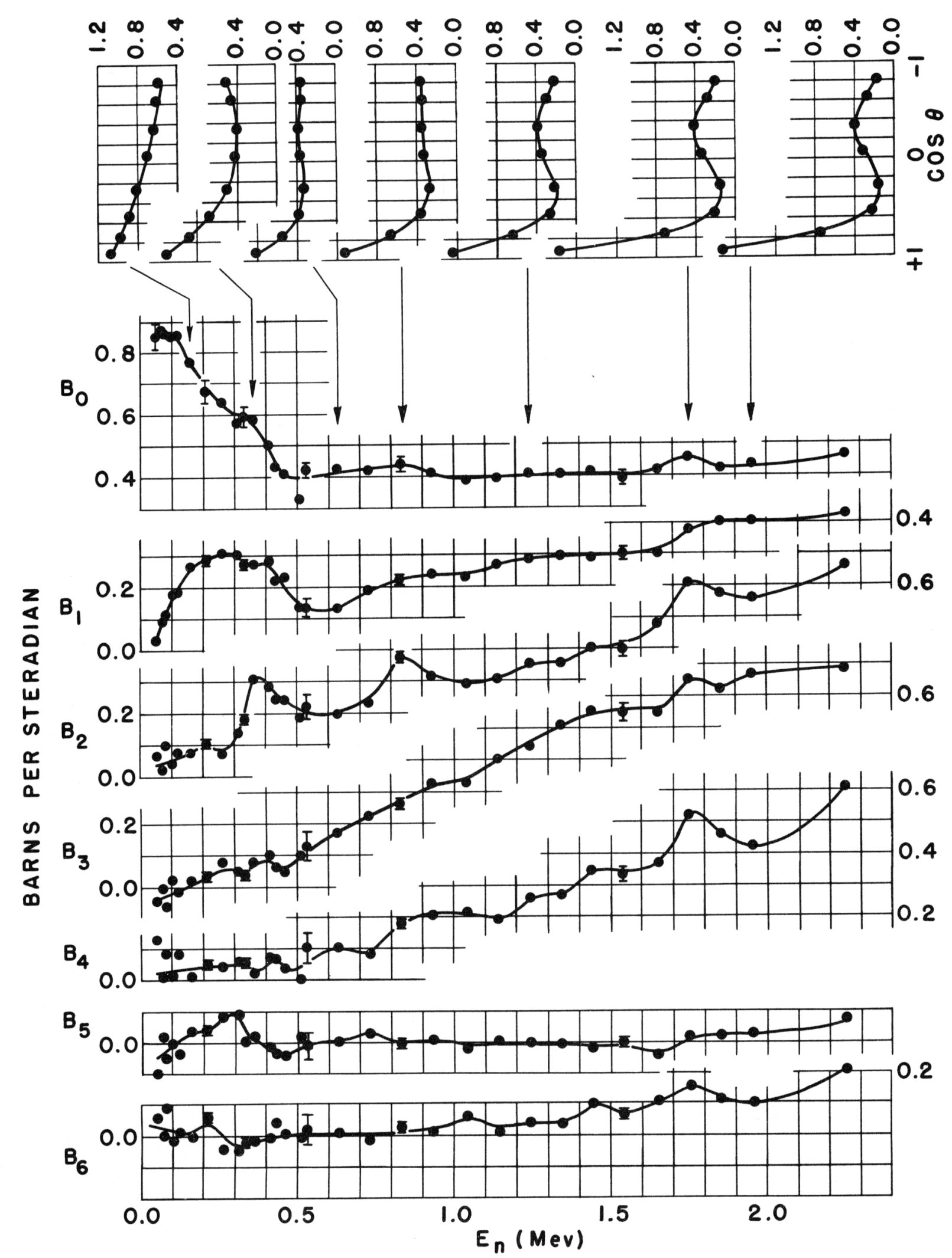

Fig. 14. The differential cross section for scattering of neutrons by $\mathrm{Pb}$. 


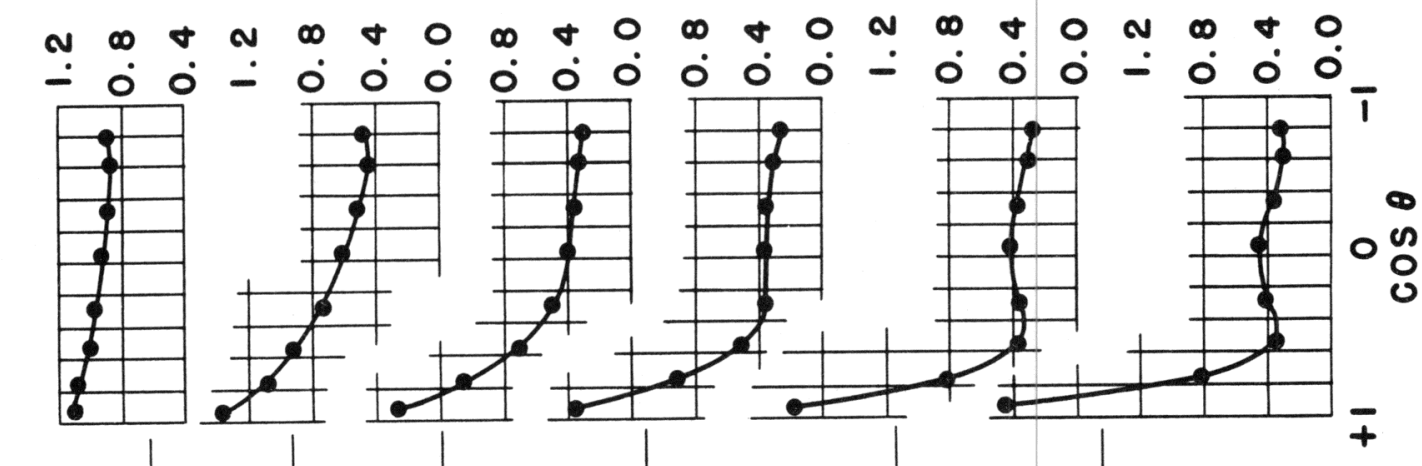

Bo

0.8

0.6
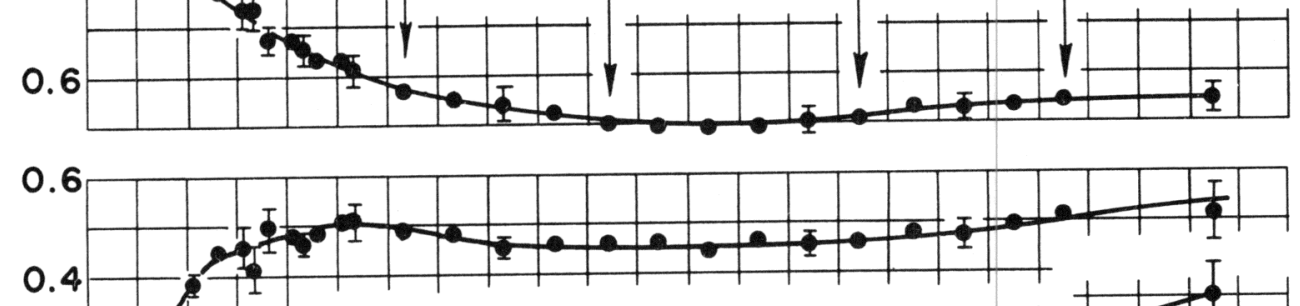

B

0.2
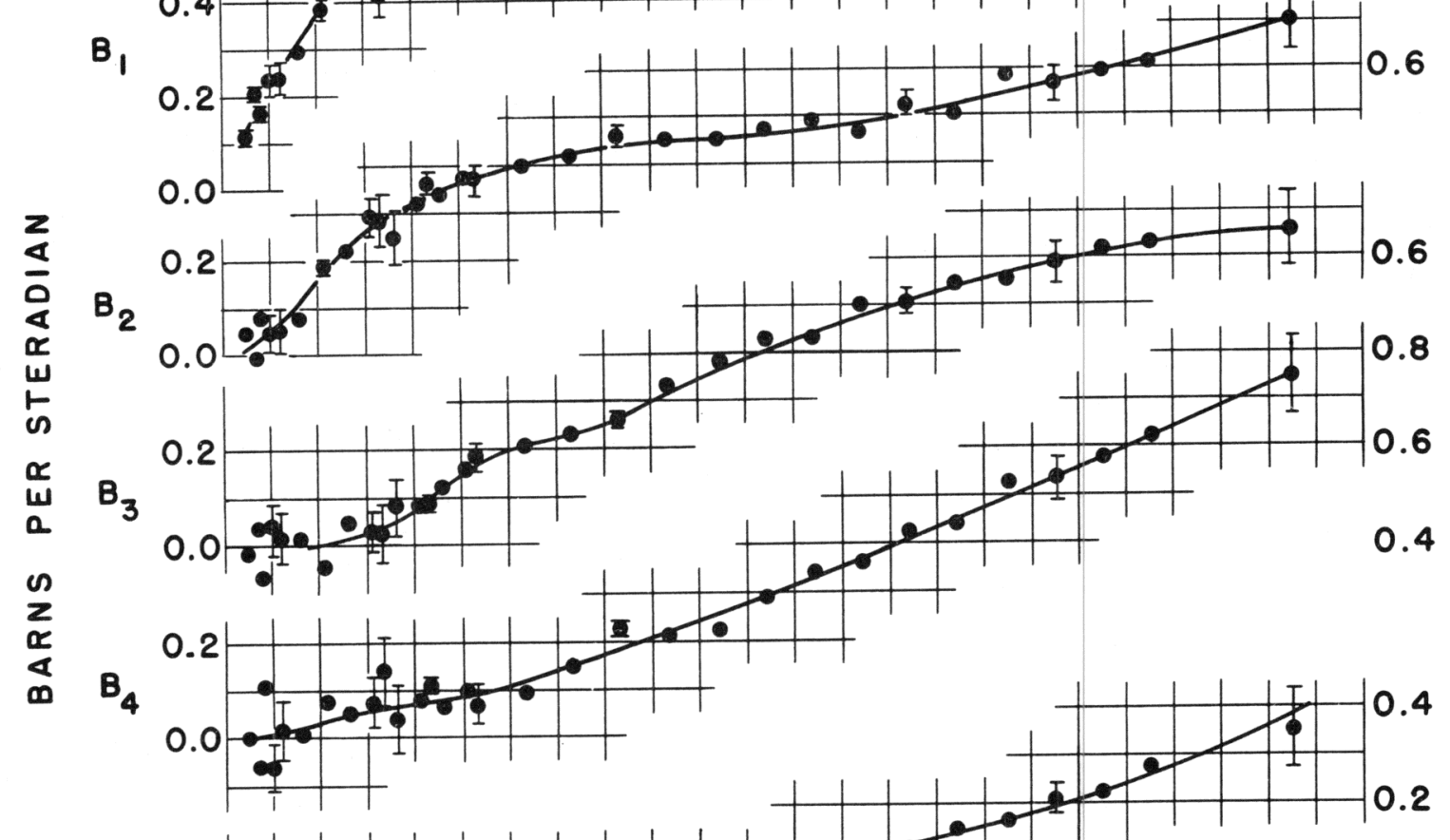

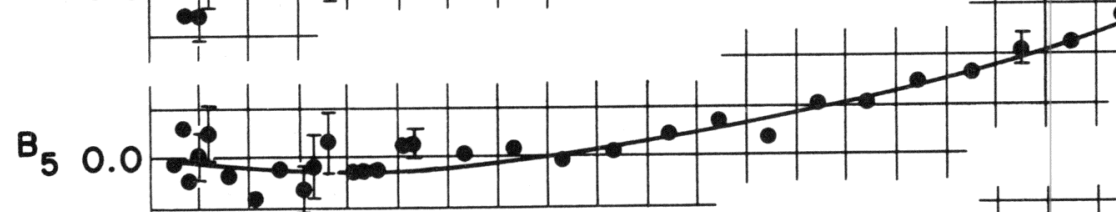

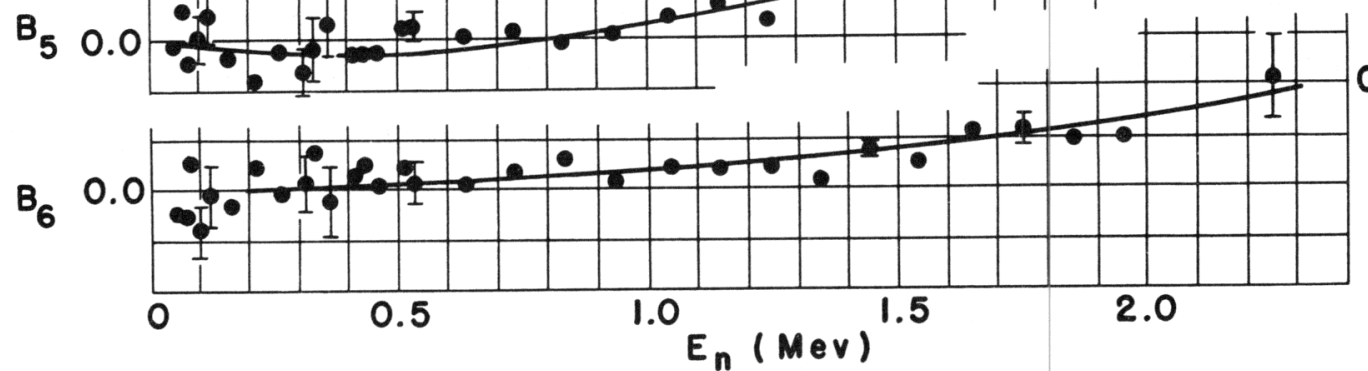

Fig. 15. The differential cross section for scattering of neutrons by U. 